\title{
Zur plastizitätstheoretischen Berechnung statisch unbestimmter Stahlbetonbalken
}

\section{Doctoral Thesis}

Author(s):

Bachmann, Hugo

Publication date:

1967

Permanent link:

https://doi.org/10.3929/ethz-a-000150808

Rights / license:

In Copyright - Non-Commercial Use Permitted 
Diss. Nr. 4069

\title{
Zur plastizitätstheoretischen Berechnung statisch unbestimmter Stahlbetonbalken
}

\author{
ABHANDLUNG
}

zur Erlangung

der Würde eines Doktors der technischen Wissenschaften

der

EIDGENOSSISCHEN TECHNISCHEN HOCHSCHULE ZURICH

vorgelegt von

HUGO BACHMANN

dipl. Bauingenieur ETH

geboren am 27. September 1935

von Niedermuhlern (Kt. Bern)

Angenommen auf Antrag von

Prof. Dr. B. Thürlimann, Referent

Prof. E. Amstutz, Korreferent

Juris Druck + Verlag Zürich

r967 


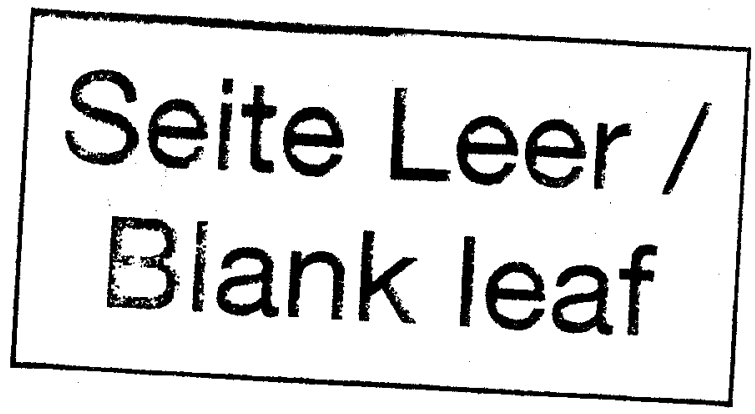


Meiner Gattin

und

meinen Eltern gewidmet 


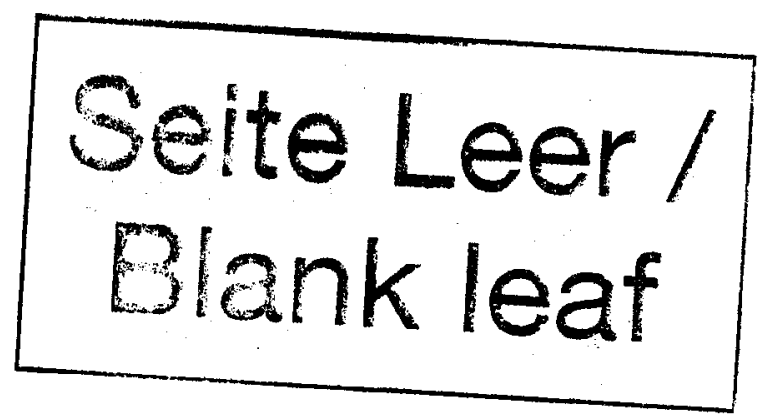


Bei der plastizitätstheoretischen Berechnung von statisch unbestimmten Tragwerken wird im allgemeinen eine genügende Verformbarkeit des betreffenden Baustoffes in plastifizierten Bereichen, sogenannten plastischen Gelenken, vorausgesetzt. Diese Annahme trifft bei Stahlbetontragwerken nicht immer zu. Gelegentlich tritt ein Bruch ein, bevor eine vollständige Umlagerung der innern Kräfte erfolgt ist, da der Baustoff Stahlbeton ziemlich spröde ist.

Der Zweck dieser Arbeit ist, bei statisch unbestimmten Stahlbetonbalken die Verformungen in plastischen Gelenken zu untersuchen. Es wird versucht, Modelle und Verfahren zu entwickeln, mit denen das Verformungsvermögen solcher Gelenke beurteilt werden kann. Zudem sollten aus den Verformungen des ganzen Tragwerkes die zum Erreichen der Traglast erforderlichen Gelenkwinkel ermittelt werden können.

Herrn Prof. Dr. B. Thürliman möchte ich für die stets grosszügige Förderung dieser Arbeit herzlich danken. Herrn Prof. E. Amstutz gebührt mein bester Dank für die Uebernahme des Korreferates.

Die vorliegende Arbeit wurde ermöglicht durch Forschungskredite der von Moos' schen Eisenwerke Luzern. Dieser Firma ist der Verfasser sehr zu Dank verpflichtet. 


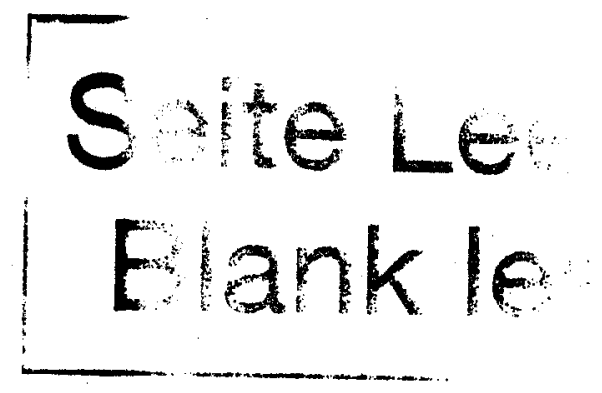


1. Einleitung

1.1 Problemstellung

1.2 Grundsätzliches zum Vorgehen

2. Das Biegeriss-Gelenk

2. 1 Modell

2.11 Definition $\quad 14$

$\begin{array}{ll}\text { 2.12 Gelenkwinkel } & 17\end{array}$

2. 2 Verformungen und Bruch im Biegeriss-Element 18

2.21 Verformungen in der Zugzone $\quad 19$

2.22 Versagen der Zugarmierung $\quad 35$

2. 23 Verformungen in der Druckzone $\quad 36$

$\begin{array}{ll}\text { 2.24 Versagen des Betons } & 42\end{array}$

2. 3 Rissabstand $\quad 46$

2.4 Kritischer Gelenkwinkel $\because \quad \vdots \quad 50$

2.41 Allgemeine Berechnung $\quad 50$

2. 42 Formeln für spezielle Grundlagen $\quad 57$

2.5 Diskussion der Ergebnisse _ . 65

2.51 Einfluss wichtiger Parameter auf die kritischen
Gelenkwinkel

2.52 Vergleiche mit Versuchsresultaten $\quad \cdots \quad 77$

2.53 Vergleiche mit üblicher Betrachtungsweise 82

2. 6 Allgemeine Beurteilung 96

2. 61 Verhalten des Biegeriss-Gelenkes 96

2.62 Momenten-Rotations-Charakteristik 96

3. Das Schubriss-Gelenk 98

3. 1 Modell $\quad 98$

3.11 Definition $\quad 98$

3.12 Gelenkwinkel $\quad 102$

3.2 Innere Kräfte 103

$3.21 \mathrm{Kräfte}$ in der Zugarmierung $\quad 104$

3.22 Kräfte in der Bügelarmierung und der Biegedruckzone 107

$\begin{array}{ll}3.23 \text { Schiefe Druckkräfte } & 108\end{array}$ 
3. 3 Kritischer Gelenkwinkel

Seite

3. 31 Verformungen der Zugzone zwischen zwei Biegeschubrissen

3. 32 Versagen der Zugarmierung

3. 33 Versagen des Betons

3. 34 Versagen der Bitgelarmierung

3. 35 Versagen des Verbundeg

124

126

3.4 Weitere Aspekte

3.41 Verlauf der Kraft in der Zugarmierung

3.42 Verlauf der Dehnungen in der Zugarmierung

3. 43 Vergleich mit üblicher Betrachtungsweise

3. 51 Verhalten des Schubriss-Gelenkes

3.52 Generelle Abhängigkeit des kritischen Gelenkwinkels in

Biegeriss - und Schubriss-Gelenken von der Schubbeanspruchung

4. Erforderliche Gelenkwinkel

4. 1 Mechanismus und Traglast

4. 2 Berechnung der Gelenkwinkel

4.21 Zustand beim Erreichen der Traglast 137

4. 22 Zustände vor dem Erreichen der Traglast 143

4.3 Besonderheiten bei Stahlbetonbalken $\quad 148$

4. 31 Rotationsbeginn $\quad 148$

4.32 Momente in den plastischen Gelenken 148

$\begin{array}{ll}\text { 4. } 33 \text { Steifigkeiten } & 150\end{array}$

4.34 Ausdehnung der plastischen Gelenke 158

5. Zusammenfassung $\quad 160$

6. Schlussbemerkung $\quad 169$

$\begin{array}{ll}\text { Anhang } & 170\end{array}$

$\begin{array}{ll}\text { Tafel } & 179\end{array}$

$\begin{array}{lr}\text { Bezeichnungen } & 180\end{array}$

Literatur-Verzeichnis $\quad 187$ 


\section{EINLEITUNG}

\section{1. Problemstellung}

Die Plastizitätstheorie (siehe zum Beispiel [1], [2]) berïcksichtigt die Tatsache, dass in statisch unbestimmten Tragwerken - bei einer Steigerung der ăusseren Lasten - bis zum Bruch bedeutende Umlagerungen der inneren Schnittkräfte eintreten können. Dabei bilden sich plastifizierte Bereiche, sogenannte plastische Gelenke aus. Während zum Beispiel die Biegemomente an den Stellen der plastischen Gelenke nicht mehr oder nur noch wenig grösser werden, können sie unter gewissen Voraussetzungen in andern, bis anhin nicht voll beanspruchten Tragwerksteilen weiterhin zunehmen. Nach vollständiger Kräfteumlagerung entstehen schliesslich so viele plastische Gelenke, dass sich im Tragwerk ein sogenannter Mechanismus ausbildet. Die entsprechende Last wird Traglast genannt. Sie ist die Höchstlast, welche das Tragwerk für die betreffende Lastanordnung aufnehmen kann.

Zur Bemessung von Tragwerken aus Stahl wird die Plastizitätstheorie bereits angewendet (zum Beispiel [3] ). In letzter Zeit sind zudem einige Arbeiten entstanden, welche die Erkenntnisse der Plastizitätstheorie auch zur Lobsung von Bemessungsproblemen bei Stahlbetontragwerken verwenden (zum Beispiel [4],

[5] ). Die entsprechenden Verfahren setzen voraus, dass in den plastischen Gelenken eine für die vollständige Umlagerung der Schnittkräfte genügende Verformbarkeit vorhanden ist. Es wird also angenommen, dass in jedem plastischen Gelenk die Verformung, welche zum Bruch führt, grösser oder mindestens gleich ist wie die für das Erreichen der Traglast erforderliche Verformung. Für ebene Stabtragwerke kann diese Bedingung wie folgt formuliert werden:

$$
\boldsymbol{\theta}_{\mathbf{k r}}=\boldsymbol{\theta}_{\mathrm{er}}
$$

$\theta_{\mathrm{kr}}$ ist der sogenannte kritische Winkel, welcher zu einem Versagen im Gelenk führt. Er kann auch mit dem Begriff "Rotationsfăhigkeit" charakterisiert werden. $\Theta_{\text {er }}$ bedeutet den im gleichen Gelenk für eine vollständige Schnittkraftumlagerung, 
das heisst für das Erreichen der Traglast, erforderlichen Gelenkwinkel.

Wahhrend bei Stahltragwerken die Bedingung ( 1 ) praktisch stets erfiull ist, gibt es bei Tragwerken aus Stahlbeton Fälle, wo die Rotationsfähigkeit nicht ausreicht, um eine vollständige Schnittkraftumlagerung zu erzielen. Der Grund liegt vor allem darin, dass der Beton ein ziemlich spröder Baustoff ist. Aber auch die an und für sich wesentlich besseren plastischen Eigenschaften der Armierungsstähle können infolge des Verbundes zwischen Stahl und Beton nicht mehr voll zur Geltung kommen, sodass gelegentlich der Stahl für ein Versagen im Gelenk massgebend ist.

Der Zweck dieser Arbeit ist, bei statisch unbestimmten Stahlbetonbalken die Verformungen in plastischen Gelenken zu untersuchen. Einerseits wird versucht, Modelle und Verfahren zu entwickeln, welche eine Beurteilung der kritischen Gelenkwinkel erlauben. Anderseits sollten aus den Verformungen des ganzen Tragwerks die zu einer Schnittkraftumlagerung erforderlichen Gelenkwinkel berechnet werden können.

Angesichts der grossen Schwierigkeiten, welche sich bei jeglichen Verformungsberechnungen im Stahlbeton einstellen, können vorwiegend nur qualitative Erkenntnisse erwartet werden. In manchen Fällen gelingt es auch, quantitativ die Grössenordnung abzuschätzen, wobei jedoch nur eine beschränkte Annäherung an die Wirklichkeit möglich sein wird. Oft können aber mit einer qualitativen Erfassung die Verhälnisse bereits genügend beurteilt werden. Es soll denn auch ein wesentliches Ziel dieser Arbeit sein, das komplizierte Verformungsverhalten des Verbundbaustoffes Stahlbeton - insbesondere im plastischen Bereich bis zum Bruch - im Sinne einer Grundlagenforschung durch eine vertiefte Einsicht in die allgemeinen Zusammenhänge etwas zu erhellen.

\subsection{Grundsătzliches zum Vorgehen}

Die Erforschung vieler Probleme aus dem Bereiche der Naturwissenschaften und der technischen Wissenschaften kann durch das folgende Ablaufschema dargestellt werden: 


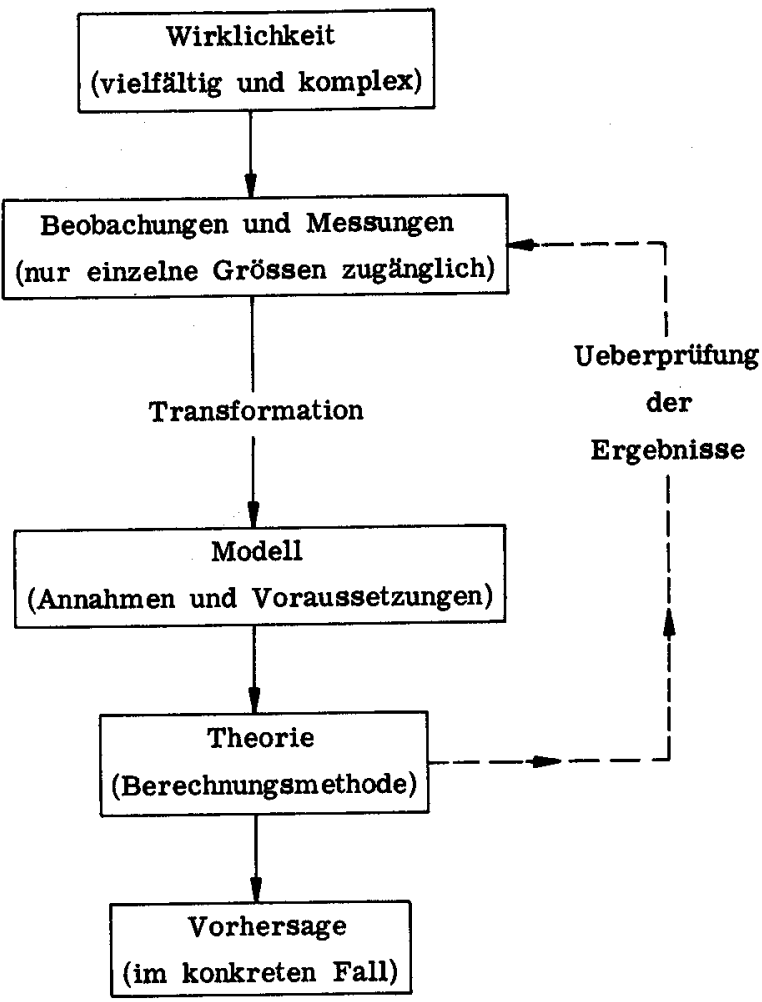

Die Wirklichkeit einer betrachteten Erscheinung ist immer ausserordentlich komplex und vielfältig. Der Mensch kann sie niemals vollumfänglich erfassen, sie ist ihm bestenfalls in Teilaspekten zugänglich. Aufmerksames Beobachten sowie das Messen einzelner, vorher zu definierender Grössen können dazu die Grundlagen liefern. Durch deren Transformation entsteht für die betrachtete Erscheinung ein sogenanntes Modell. Dieses umfasst die grundlegenden Annahmen und Voraussetzungen für eine entsprechende Theorie (Berechnungsmethode). Die Ergebnisse derselben können im Bereich der vorangegangenen Beobachtungen 
und Messungen überprift werden. Die Theorie sollte auch für ähnliche konkrete Falle eine möglichst gute Vorhersage liefern.

Der weitaus wichtigste Vorgang im Ablaufschema ist die Transformation der Beobachtungen und Messungen $\mathrm{zu}$ einem Modell. An dieses Modell sind grundsatzlich folgende Ansprïche zu stellen:

1. Das Modell soll möglichst richtig sein (das heisst möglichst naturgetreu).

2. Das Modell soll möglichst günstig sein (das heisst möglichst geeignet für eine Formulierung von Zusammenhăngen zwischen den wichtigsten Parametern).

Diese beiden Anforderungen haben meist gegensätzlichen Charakter. Wählt man das Modell möglichst richtig, dann ist es oft sehr schwierig, noch befriedigende Formulierungen angeben zu können. Wird hingegen das Modell möglichst günstig angenommen, so lässt sich daran meist schön rechnen, das heisst es können unter Umständen brillante mathematische Gebilde entwickelt werden, die jedoch durch die willkürlichen Annahmen und Voraussetzungen in Frage gestellt werden. Grundsätzlich ist natürlich dasjenige Modell das beste, dessen zugehörige Theorie die beste Uebereinstimmung mit Beobachtungen und Messungen ergibt. Da jedoch in vielen Fallen nur wenige oder uiberhaupt keine in einer Theorie vorkommende beziehungsweise daraus resultierende Grössen gemessen werden können, bleibt dem Forscher oft ein gewisser Spielraum, sein Modell mehr nach dem einen oder andern Anspruch auszurichten.

Der Verfasser bekennt sich eindeutig zum Bestreben, stets mit möglichst richtigen, also naturgetreuen Modellen arbeiten zu wollen. Er glaubt, dass vor allem dieser Weg die Möglichkeit bieten kann, eine bessere Einsicht in komplizierte Zusammenhänge zu gewinnen.

In der vorliegenden Arbeit werden für die plastischen Stahlbeton-Gelenke die Modelle "Biegeriss-Gelenk" und "Schubriss-Gelenk" entwickelt. Diese beiden Begriffe wurden erstmals in [6] verwendet. Vor allem auf Grund der Versuche [7] und [8] drängte sich die Einsicht auf, dass die üblichen Grundlagen der Stahlbetontheorie, wie die Hypothese vom Ebenbleiben der Querschnitte, der Begriff der Krïmmung, und so weiter (ganz abgesehen vom Hooke'schen Gesetz!), 
nicht geeignet sind, um die komplizierten Verformungsprobleme etwas klären zu können. Diese Grundlagen entstammen der Elastizitätstheorie für rissefreie Baustoffe mit kontinuierlichem Formänderungsverhalten. Auf Stahlbeton angewendet, muss im oberwähnten Sinne von einem ausgesprochen günstigen, jedoch nur wenig richtigen Modell gesprochen werden. Denn beim Stahlbeton handelt es sich infolge der Rissebildung um einen Baustoff mit ausgeprägt diskontinuierlichem Formănderungsverhalten. Dieses kann mit Begriffen aus der Elastizitătstheorie nicht befriedigend erfasst werden. Bereits bei der Schubbemessung von Spannbetontragwerken hat sich gezeigt, dass die unbesehene Uebernahme von Vorstellungen aus der Elastizitätstheorie zu ungenügenden und unter Umständen gefăhrlichen Ergebnissen führen kann (siehe [9]). So wird denn hier der Versuch unternommen, mit den Modellen "Biegeriss-Gelenk" und "Schubriss-Gelenk" auf neuartiger Grundlage an die Probleme heranzugehen. 


\section{DAS BIEGERISS-GELENK}

\section{1. Modell}

\subsection{Definition}

In Bild 1 ist schematisch ein Biegeriss-Gelenk dargestellt. In einem solchen Gelenk sollen definitionsgemăss nur Biegerisse vorhanden sein, das heisst Risse, welche vom Zugrand her senkrecht zur Stabaxe bis zur Biegedruckzone verlaufen. Allfallige kürzere Risse, wie sie gelegentlich zwischen den "Hauptrissen" auftreten können und welche meist nur den Bereich der Zugarmierung umfassen, werden vernachlässigt, da sie an die Verformungen des Gelenkes nur einen unbedeutenden Beitrag leisten. Typische Biegeriss-Gelenke sind in den Arbeiten

[6] und [7] abgebildet.
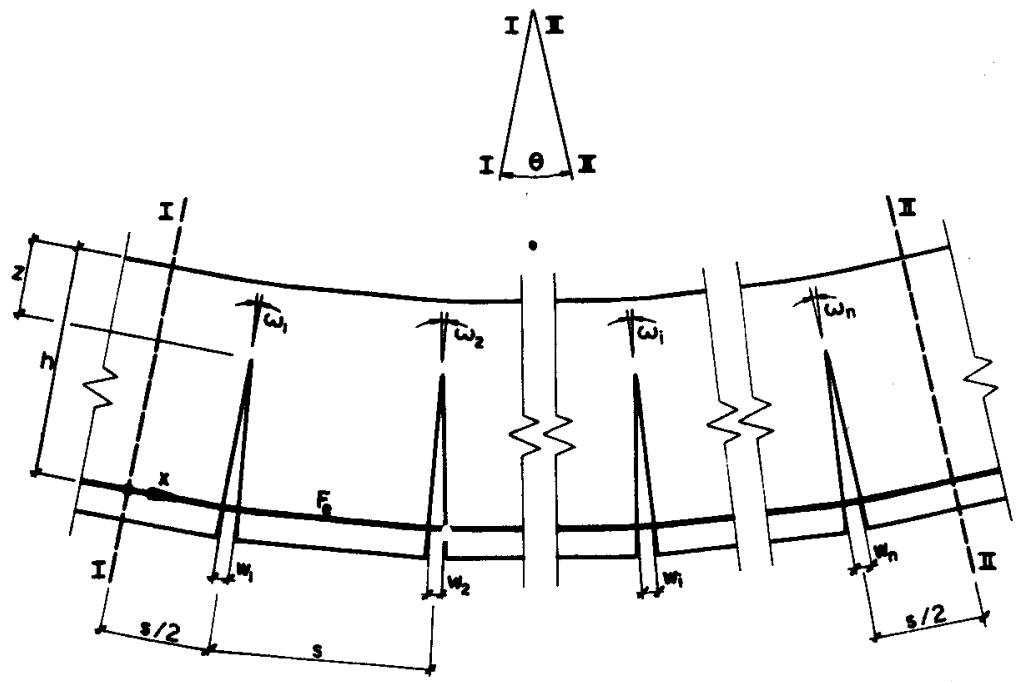

Bild 1

Modell des Biegeriss-Gelenkes 
Im weiteren wird vorausgesetzt, dass innerhalb des Gelenkes die Art der Zugarmierung (Querschnitt, Stabdurchmesser, Spannungs-Dehnungs-Diagramm, Verbundeigenschaften) sowie der Balkenquerschnitt sich nicht verändern. Das plastische Gelenk soll diejenigen Risse umfassen, in welchen plastische Deformationen der Zugarmierung auftreten. Sind $\varepsilon_{\mathrm{eR}}$ die Dehnung der Zugarmierung in einem Rissequerschnitt und $\varepsilon_{\mathrm{f}}$ deren nominelle Fliessdehnung nach Bild 2, so gilt in allen Rissen des Gelenkes:

$$
\varepsilon_{\mathrm{eR}} \geqslant \varepsilon_{\mathrm{f}}
$$

Ferner muss in allen Rissen das Biegemoment $M$ mindestens gleich dem Rissmoment $M_{R}$ sein:

$$
\begin{aligned}
& \mathbf{M} \quad \geq \mathbf{M}_{\mathbf{R}} \\
& \mathbf{M}_{\mathbf{R}} \quad=\mathrm{W} \cdot \boldsymbol{\beta}_{\mathrm{bz}}
\end{aligned}
$$

$\beta_{\mathrm{bz}}$ bedeutet die Biegezugfestigkeit des Betons, $W$ das Widerstandsmoment des ungerissenen Querschnitts. Mit den Bedingungen ( 2 ) und ( 3 ) ist die Ausdehnung des Gelenkes eindeutig festgelegt.

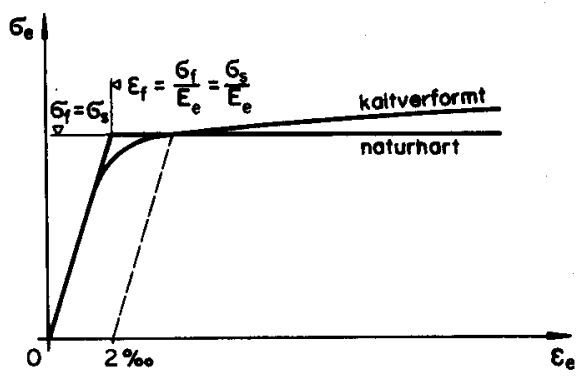

Bild 2

Definition der nominellen Fliessspannung und Fliessdehnung des Armierungsstahles 
Der Abstand s der Biegerisse wird als konstant angenommen und seine Grösse vorlăfig als bekannt vorausgesetzt. Regeln für dessen Bestimmung werden unter 2.3 angegeben.

Der Abstand $z$ des Rissendes von der Druckkante ist grundsătzlich vom Verformungszustand im betreffenden Rissebereich abhängig (vergleiche zum Beispiel [7] ). Nach Ueberschreiten der Streckgrenze $\sigma_{s}$ in der Zugarmierung (Bild 2) verringert sich $z$ jedoch nur noch wenig und strebt bald einem Mindestwert zu. Dieser kann genügend genau mittels einer Gleichgewichtsbedingung aus den ungefăhren Kräften ermittelt werden, welche im Rissequerschnitt angreifen (Bild 3).
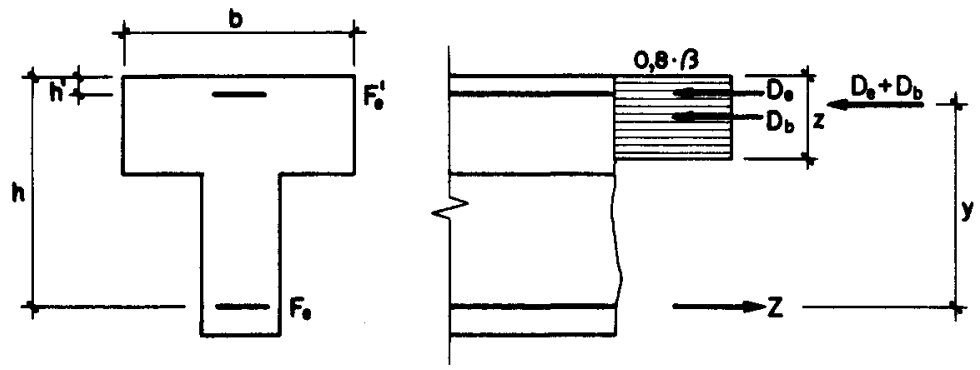

\section{Bild 3}

Krăfte und geometrische Grössen Im Querschnitt eines Biegerisses

Dazu sei vereinfachend die Spannung in der Zug- und Druckarmierung gleich der nominellen Fliessspannung $G_{f}$ beziehungsweise $\sigma_{f}$ gesetzt. Ferner wird die Betonspanmung über die Hơhe $z$ der Blegedruckzone mit einem Mittelwert von $0,8 \beta$ als gleichmăssig verteilt angenrmmen. Damit wird für eine gleichbleibende Breite $b$ der Druckzone:

$$
z=\frac{h}{\delta, 8 \beta} \cdot\left(\mu \sigma_{f}-\mu^{\prime} G_{f}^{\prime}\right)
$$


In diesem Ausdruck kommen die wesentlichsten Grössen, welche $\mathrm{z}$ beeinflussen, vor. Die zusätzliche Einführung einer Verträglichkeitsbedingung, zum Beispiel aufgrund der Hypothese vom Ebenbleiben der Querschnitte im Zusammenhang mit einer Betonbruchstauchung, würde angesichts der dabei nötigen Annahmen und Voraussetzungen nur eine scheinbare Verbesserung der Genauigkeit bewirken. Immerhin ist stets zu prüfen, ob

$$
\mathbf{z}=\mathbf{h}^{\prime} \text {. }
$$

Ist dies nicht der Fall, was nur bei Verhältnissen $\mu / \mu \rightarrow 1,0$ sowie abnormal grossen Werten $h^{\prime} / h$ vorkommen kann, so ist $z=h^{\prime}$ anzusetzen.

Ferner ergibt sich noch der Hebelarm der inneren Kräfte (Bild 3) welcher weiter hinten benötigt wird:

$$
y=h-\frac{z}{2}+\frac{\mu^{\prime}}{\bar{\mu}} \cdot \frac{G_{f}^{\prime}}{\sigma_{f}} \cdot\left(\frac{z}{2}-h^{\prime}\right)
$$

\subsection{Gelenkwinkel}

Der Gelenkwinkel $\Theta$ in einem Biegeriss-Gelenk wir als gegenseitige Verdrehung der Betonquerschnitte I - I und II - II gemäss Bild 1 definiert. Diese Endquer schnitte sollen je den Abstand $s / 2$ von den äussersten beiden Rissen des plastischen Gelenkes haben. $\Theta$ kann mit den üblichen geometrischen Năherungen ausgedrückt werden als Quotient der Formänderungen auf der Höhe der Zugarmierung und dem Abstand der Zugarmierung vom Rissende. Sind im Gelenk n Risse vorhanden und bezeichnet $w_{i}$ die jeweilige Rissweite auf der Höhe der Zugarmierung. so heisst dies:

$$
\theta=\frac{\sum_{1}^{n} w_{i}+\int_{I}^{n} \varepsilon_{b} d x}{h-z}
$$


Da der von den Betondehnungen $\varepsilon_{b}$ zwischen den Rissen herrührende Anteil an den Verformungen gegenüber den Rissweiten $w_{i}$ ausserst gering ist, wird

$$
\theta=\frac{\sum_{1}^{n} w_{i}}{h-z}=\sum_{1}^{n} \omega_{i},
$$

worin wir

$$
\omega_{i}=\frac{w_{i}}{h-z}
$$

als "Risswinkel" bezeichnen wollen.

Der Gelenkwinkel im Biegeriss-Gelenk ist somit gleich der Summe der einzelnen Risswinkel.

\section{2. Verformungen und Bruch im Biegeriss-Element}

Vorerst werden die Verformungen und der Bruch im Bereich eines einzelnen Biegerisses betrachtet. Dieser Bereich wird "Biegeriss-Element" genannt. Dessen Endquerschnitte befinden sich in der Mitte zwischen einem Biegeriss und dessen benachbarten Rissen, sodass die Elementlänge gleich dem Rissabstand $\mathbf{s}$ ist. Bild 4 zeigt ein solches Biegeriss-Element, welches ausschliesslich durch ein Biegemoment beansprucht sei. Die Erweiterung auf mehrere Elemente beziehungsweise auf ein ganzes Biegeriss-Gelenk, unter Berücksichtigung einer gleichzeitig wirkenden Querkraft, erfolgt später. 


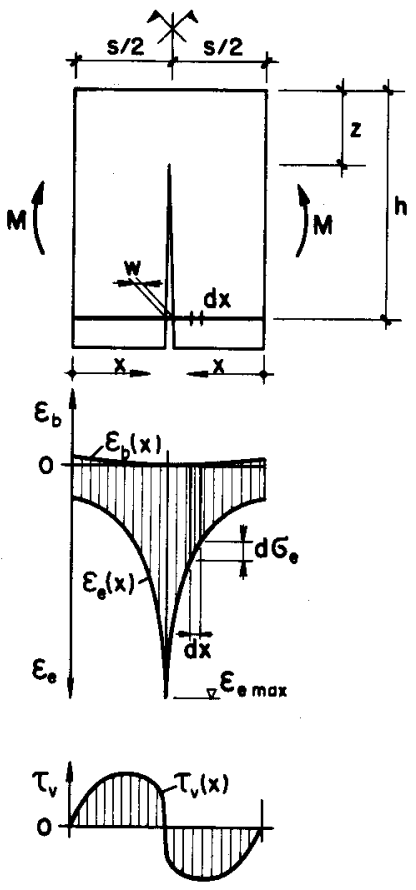

\section{Bild 4}

Bereich eines einzelnen Biegerisses ("Biegeriss-Element"); genereller Verlauf der Stahlund Betondehnungen sowie der Verbundspannungen bei reiner Biegung

\subsection{Verformungen in der Zugzone}

Im betrachteten Bereich eines einzelnen Biegerisses interessiert insbesondere der Zusammenhang zwischen der Rissweite und den übrigen Verformungen der Zugzone.

\section{a) Allgemeine Zusammenhänge}

In Bild 4 ist der generelle Verlauf der Stahldehnungen $\varepsilon_{e}$ und der Betondehnungen $\varepsilon_{b}$ längs der Zugarmierung aufgetragen. Unter der Voraussetzung, dass für die Zugarmierung stets

$$
\frac{d \sigma_{e}}{d \varepsilon_{e}} \geqslant 0 \text {, }
$$


erreicht sowohl die Spannung wie auch die Dehnung derselben im Rissequerschnitt einen Höchstwert. Durch die Wirkung der weiter unten definierten Verbundspannungen verringern sie sich mit zunehmender Entfernung vom Riss. Anderseits ist die Betondehmung am Rand des Risses Null und steigt gegen die Mitte zwischen den Rissen hin an. Die Rissweite w auf der Höhe der Zugarmierung ergibt sich aus der Differenz der Verformungen des Stahles und des Betons:

$$
w=2 \cdot\left[\int_{0}^{s / 2} \varepsilon_{e}(x) d x-\int_{0}^{s / 2} \varepsilon_{b}(x) d x\right]
$$

In Anbetracht der grossen plastischen Verformungen des Stahles ist der Beitrag der Betondehnungen an die Rissweite verschwindend klein. Deshalb kann w wie folgt angeschrieben werden:

$$
w=2 \cdot \int_{0}^{s / 2} \varepsilon_{e}(x) d x=\kappa \cdot s \cdot \varepsilon_{e \max }
$$

Darin bedeutet $\varepsilon_{\text {e } \max }$ die maximale Stahldehnung. Die Grösse $\kappa$ definieren wir als Verbundkoeffizienten. Dieser ist gleich dem Verhältnis der in Bild 4 über der Funktion $\varepsilon_{e}(x)$ liegenden schraffierten Fläche zur Fläche des umschriebenen Rechtecks. $\kappa$ kann auch als Quotient der mittleren Stahldehnung

$$
\varepsilon_{\mathrm{em}}=\frac{\mathrm{w}}{\mathrm{s}}
$$

und der maximalen Stahldehnung $\varepsilon_{\text {e max }}$ aufgefasst werden, oder als Verhältnis einer Strecke $\kappa \cdot s$, längs welcher das volle $\varepsilon_{\mathrm{e} \max }$ vorhanden sein müsste (daneben $\varepsilon_{e}=0$ ), zum Rissabstand s. Somit gilt:

$$
\kappa=\frac{2 \int_{0}^{s / 2} \varepsilon_{e}(x) d x}{s \cdot \varepsilon_{e \max }}=\frac{\varepsilon_{e m}}{\varepsilon_{e \max }}
$$


Die Grösse von $\kappa$ variiert je nach Umständen zwischen etwa 0,10 und 1,0 .

Der Verlauf der Stahldehnungen $\varepsilon_{e}$ hängt von verschiedenen Einflussgrössen $a b$. Ist $\varepsilon_{e}(x)$ für gegebene Randbedingungen bekannt, so kann der Verbundkoeffizient bestimmt werden und der gesuchte Zusammenhang zwischen der Rissweite und den Beanspruchungen und Verformungen in der Zugzone ist gefunden.

Wie bereits dargelegt, ist die Beanspruchung der Zugarmierung im Rissequerschnitt am grössten. Neben dem Riss wird die Zugkraft reduziert durch den Verbund der Armierungsstäbe mit dem umgebenden Beton. Dieser Verbund beruht auf der Verzahnung der unebenen, meist profilierten Staboberfläche mit dem Betonmörtel. Dabei kommen Haftkräfte, Reibungskräfte sowie vor allem Druckkräfte von Rippen und dergleichen auf den Beton zur Wirkung. Die Gesamtheit dieser im einzelnen recht komplizierten Effekte kann vereinfachend als eine am differentiellen Element der Staboberfläche wirkende nominelle Verbundspannung $\tau_{\mathrm{v}}$ definiert werden.

Ueber die Grösse und den Verlauf der Verbundspannungen (auch "Haftspannungen" genannt) längs der Zugarmierung zwischen den Rissen von Biegebalken sind in der Literatur die verschiedensten Annahmen zu finden (zum Beispiel [10]). Die Verteilung wird beispielsweise dreieck-, rechteck-, parabel- oder sinusförmig angenommen, wobei die Höhe der maximalen Verbundspannung (oft als "Haftfestigkeit" bezeichnet) recht unterschiedlich angesetzt wird. Diese Annahmen mögen in speziellen Fällen mehr oder weniger zutreffen. Im allgemeinen hängt jedoch die Grösse der Verbundspannung an einer bestimmten Stelle eines Stabes ausser von dessen Oberflächenbeschaffenheit und den Betoneigenschaften vor allem vom Ausmass der Verschiebung zwischen Stabelement und Beton ab. Bereits W. Djabry hat in der grundlegenden Untersuchung [11] auf diese Tatsache hingewiesen. G. Rehm hat dann in [12] den Zusammenhang zwischen örtlicher Verbundspannung und zugehöriger örtlicher Verschiebung am differentiellen Stabelement als "Grundgesetz des Verbundes" bezeichnet. Ist dieses "Verbundgesetz" bekannt, so erhält man bei längs eines Stabes gegebener Verschiebung den Verlauf der Verbundspannungen.

Das Verbundgesetz kann auf einfache Weise experimentell an Probekörpern gemäss Bild 5 ermittelt werden. 


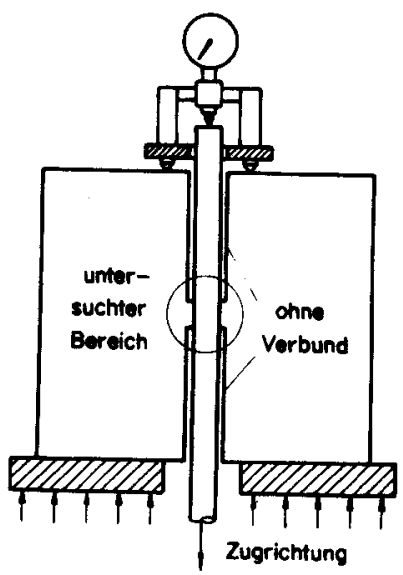

Bild 5

Versuchsanordnung zur Ermittlung des Verbundgesetzes nach G. Rehm [12]

Die Stahlstäbe werden in Betonwürfel von zum Beispiel 10 oder $20 \mathrm{~cm}$ Kantenlänge mittig einbetoniert. Die eigentliche Verbundlänge beträgt etwa das 1 -fache des Stabdurchmessers, wobei auf die Art einer allfälligen Profilierung (zum Beispiel Rippenabstand) Rücksicht zu nehmen ist. Durch genaue Messung der

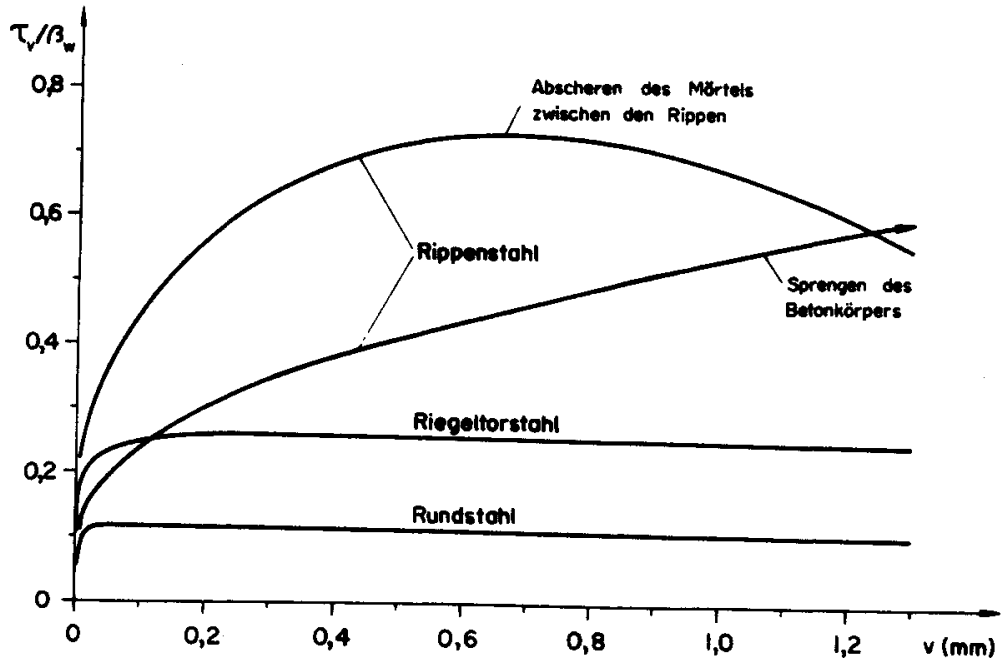

Bild 6

Typische Verbundgesetze für stehend einbetonierte Stäbe nach G. Rehm [12] 
Stabzugkraft und der Verschiebung des unbelasteten Stabendes erhält man sozusagen am differentiellen Stabelement den gesuchten Zusammenhang zwischen Verbundspannung und Verschiebung.

In Bild 6 sind typische Verbundgesetze für drei verschiedene Staboberflächen dargestellt [12]. Auf der Ordinate ist die auf die Würfeldruckfestigkeit $\beta_{w}$ bezogene Verbundspannung $\tau_{\mathrm{v}}$ abgetragen. Die Abszisse bedeutet die Verschiebung $v$ in $\mathrm{mm}$. Bei nicht profilierten Rundstählen bleibt die Verbundspannung über einen verhältnismässig grossen Verschiebungsbereich nahezu konstant, wobei der Höchstwert stark von der Oberflächenbeschaffenheit abhängt (glatt, vernarbt, rostig). Riegeltorstahl mit Spiralrippen und parallel zur Stabaxe angeordneten Riegeln zeigt, was die Form des Verbundgesetzes betrifft, ein ähnliches Verhalten wie Rundstahl. Schon bei recht kleinen Verschiebungen von $0,1 \mathrm{~mm}$ wird bereits etwa $80 \%$ des Höchstwiderstandes erreicht. Dieser ist jedoch bedeutend grösser als bei Rundstahl. Beim Rippenstahl sind zwei Formen der Verbundgesetze zu unterscheiden. Die eine Form ist durch eine stets ansteigende Linie gekennzeichnet. Sie gilt für den Fall, dass der Verbund schliesslich durch Sprengen des Betonkörpers zerstört wird. Bei der zweiten Form werden beim Erreichen des Höchstwertes die Mörtelteile zwischen den Rippen abgeschert, worauf eine Abnahme der Verbundspannungen eintritt. Sowohl die Form des Verbundgesetzes wie auch der Höchstwert der Verbundspannungen werden von der Höhe und dem Abstand der Rippen beeinflusst. Bei stark gerippten Stäben liegen die Höchstwerte deutlich über denjenigen von Riegeltorstahl.

Bei sämtlichen Oberflächenarten spielt die Lage der Stäbe beim Betonieren eine Rolle. Die in Bild 6 gezeigten Verbundgesetze wurden bei stehender Einbettung ermittelt. Bei liegender Einbettung ist die höchste Verbundspannung im allgemeinen kleiner. Die Reduktion kann bei Rundstählen 50 bis $70 \%$ betragen, während sie bei Rippenstählen geringer ist und vor allem von der Rippenhöhe abhängt. Je nach Art der Profilierung kann das Verbundgesetz auch durch die Grösse des Stabdurchmessers beeinflusst werden, sodass unter Umständen für verschiedene Stabdurchmesser andere Verbundgesetze gelten.

Durch die Einführung des Verbundgesetzes nimmt man an, dass die Grösse der Stahlbeanspruchung im betrachteten Stabelement keinen Einfluss auf die Verbund- 
spannung hat. Demgegenüber hat W. Djabry [11] gezeigt, dass hier infolge der Querkontraktion des Stabes eine gewisse Abhängigkeit bestehen kann. Diese macht sich allerdings nur bei glatten Rundstăhlen bemerkbar und ist auch dort gegenüber den mit dem Verbundgesetz erfassten Einflüssen vernachlässigbar klein.

Für die Verteilung der Spannungen und Dehnungen in der Zugzone spielt jedoch nicht nur die örtlich wirkende Verbundspannung eine Rolle. Wie aus den noch folgenden Darlegungen hervorgehen wird, ist vor allem dort, wo der Stahl über die Proportionalitätsgrenze $\varepsilon_{\text {plast }}=0,05 \%$ hinaus beansprucht wird, auch der Zusammenhang zwischen Spannung und Dehnung in der Zugarmierung von Bedeutung.

Somit bilden das Verbundgesetz

$$
\tau_{v}=\tau_{v}(v)
$$

und das Spannungs-Dehnungs-Diagramm des Stahles

$$
\varepsilon_{e}=\varepsilon_{e}\left(\sigma_{e}\right)
$$

die Grundlagen, mit denen in einem Biegeriss-Element bei gegebenen Anfangswerten der Verlauf der Funktion $\varepsilon_{e}(x)$ und damit die Spannungen und Dehnungen in der Zugzone sowie deren Zusammenhang mit der Rissweite bestimmt werden kann.

Innerhalb der Strecke $d x$ (Bild 4) bewirken die Verbundspannungen die folgende Aenderung der Zugkraft eines Armierungsstabes:

$$
\mathbf{d} \sigma_{e} \cdot \mathbf{F}=\tau_{v}(v) u d x
$$

Daraus wird:

$$
\frac{d \sigma_{e}}{d x}=\tau_{v}(v) \cdot \frac{4}{d}
$$


Anderseits ist die Aenderung der Relativverschiebung zwischen Stahl und Beton gleich der Längenänderung des Stabelementes dx:

$$
d v=\varepsilon_{e}(x) d x
$$

Da die Gleichung

$$
\varepsilon_{e}(x)=\varepsilon_{e}\left(\sigma_{e}\right)
$$

stets erfullt sein muss, wird aus ( 18 ):

$$
\frac{d v}{d x}=\varepsilon_{e}\left(\sigma_{e}\right)
$$

G. Rehm hat in [12] eine der Gleichung ( 20) entsprechende Beziehung unter Beschränkung auf elastisches Stahlverhalten aufgestellt und die sich in Kombination mit ( 17 ) ergebende Differentialgleichung für spezielle Ansätze für das Grundgesetz analytisch gelöst. Bei der hier gestellten Aufgabe befindet man sich jedoch normalerweise im Bereich plastischer Stahldehnungen. Auch sollte jedes beliebige Verbundgesetz verarbeitet werden können. Eine Lösungsmöglichkeit ergibt sich durch die Umwandlung der Differentialausdrücke ( 17 ) und ( 20 ) in Differenzenbeziehungen:

$$
\begin{aligned}
& \Delta \sigma_{e}=\tau_{v}(v) \cdot \frac{4}{d} \cdot \Delta x \\
& \Delta v=\varepsilon_{e}\left(\sigma_{e}\right) \cdot \Delta x
\end{aligned}
$$

Hiermit kann ein Integrationsschritt iber einen endlichen Stababschnitt $\Delta x$ als Iteration durchgefüht werden. Dazu wird vorerst angenommen, dass an einer bestimmten Stelle die Verschiebung $v_{i}$ sowie die Stahlspannung $G_{e i}$ bekannt sind. Damit kennt man aus dem Verbundgesetz auch die Verbundspannung $\tau_{v i}$ und aus dem Spannungs-Dehnungs-Diagramm die Stahldehnung $\varepsilon_{\mathrm{e}}$ (siehe Bild 7). 

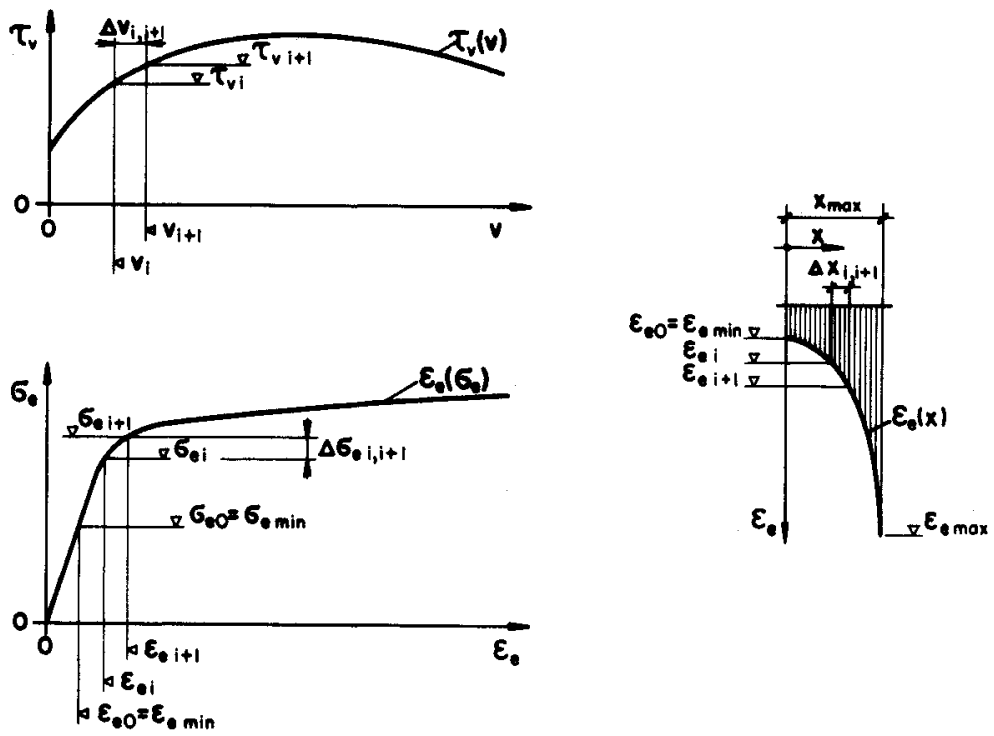

\section{Bild 7}

Zur schrittweisen Berechnung der Funktion $\varepsilon_{\mathrm{e}}(\mathrm{x})$

Es sind somit gegeben:

$$
\begin{array}{ll}
v_{i} ; & \tau_{v i}=\tau_{v}\left(v_{i}\right) \\
\sigma_{e i} ; & \varepsilon_{e i}=\varepsilon_{e}\left(\sigma_{e i}\right)
\end{array}
$$

Der Integrationsschritt über das Stabelement $\Delta x_{1, i+1}$ ergibt sich wie folgt:

$$
\Delta G_{e i, i+1}=\tau_{v i, i+1} \cdot \frac{4}{d} \cdot \Delta x_{i, i+1}
$$

$\tau_{v i, i+1}$ ist vorerst zu schätzen. Weiter wird: 


$$
\begin{gathered}
\sigma_{e i+1}=G_{e i}+\Delta G_{e i, i+1} ; \varepsilon_{e i+1}=\varepsilon_{e}\left(G_{e i+1}\right) \\
\Delta v_{i, i+1}=\varepsilon_{e}\left(G_{e i, i+1}\right) \cdot \Delta x_{i, i+1}
\end{gathered}
$$

Darin ist:

$$
\varepsilon_{e}\left(\sigma_{e i, i+1}\right)=\frac{1}{\Delta \sigma_{e i, i+1}} \cdot \int_{\sigma_{e i}}^{\sigma_{e i+1}} \varepsilon_{e}\left(\sigma_{e}\right) d \sigma_{e} \cong \frac{\varepsilon_{e i}+\varepsilon_{e i+1}}{2}
$$

Ferner:

$$
v_{i+1}=v_{i}+\Delta v_{i, i+1} ; \quad \tau_{v i+1}=\tau_{v}\left(v_{i+1}\right)
$$

Kontrolle:

$$
\tau_{v i, i+1}=\frac{1}{\Delta v_{i, i+1}} \cdot \int_{v_{i}}^{v_{i+1}} \tau_{v}(v) d v \cong \frac{\tau_{v i}+\tau_{v i+1}}{2}
$$

Zeigen sich wesentliche Unterschiede zwischen dem berechneten und dem eingangs geschätzten Wert von $\tau_{v i, i+1}$, so ist die Rechnung mit einem verbesserten Wert zu wiederholen. Die Konvergenz der Iteration hängt vor allem von der Schrittgrösse $\Delta \mathbf{x}$ und der Funktion

$$
\frac{d \tau_{v}(v)}{d v}
$$

ab. Nach genügender Uebereinstimmung kann die Integration über das nächste Stabelement $\Delta x_{i+1}, i+2$ vorgenommen werden, und so weiter.

Als Resultat der Berechnung ergeben sich die Funktionen $\sigma_{e}(x), \varepsilon_{e}(x), v(x)$ und $\tau_{\mathbf{v}}(\mathbf{x})$. 
Es besteht hier allerdings ein "Anfangswertproblem", da oft an keiner Stelle innerhalb des Biegeriss-Elementes sowohl die Verschiebung $\mathrm{v}$ als auch die Stahlspannung $\sigma_{e}$ (oder die Dehnung $\varepsilon_{e}$ ) bekannt sind. In den meisten Fallen (Ausnahmen vergleiche 3.35) muss jedoch trgendwo zwischen zwei benachbarten Rissen die Relativverschiebung $\mathbf{v}$ zwischen Zugarmierung und Beton verschwinden. Somit kann gesetzt werden:

$$
\nabla(x=0)=v_{0}=0
$$

An der so definierten Stelle $x=0$ wechselt die Verbundspannung $\tau_{v}$ das Vorzeichen. Von hier bis zum Riss, das heisst bis $x=x_{\max }$, nimmt dann die Verschiebung $v$ zu bis $z u$ ihrem Maximalwert $v_{\max }$ am Rissrand.

Für die hier betrachtete reine Biegebeanspruchung - sowie unter den übrigen getroffenen Voraussetzungen - befindet sich die Stelle $x=0$, beziehungsweise $v_{0}=0$ aus symmetrischen Gründen genau in der Mitte zwischen zwei benachbarten Rissen. Es gilt:

$$
x_{\max }=\frac{s}{2}
$$

Würde man auch die dortige Stahlspannung

$$
\sigma_{e}(x=0)=G_{e}
$$

kennen, so kőnnte die Berechnung mit bekannten Anfangswerten von $x=0$ bis $\mathrm{zu}$ $x=x_{\max }=\frac{s}{2}$ durchgefuhrt werden. Mit dem Wert $v_{\max }$, beziehungsweise dem Integral der Funktion $\varepsilon_{\mathrm{e}}(\mathbf{x})$,

$$
v_{\max }=\int_{0}^{s / 2} \varepsilon_{e}(x) d x=\frac{w}{2},
$$

liessen sich die Rissweite $w$ sowie der Verbundkoeffizient $k$ nach Gleichung (15) bestimmen. 
- $29-$

Zum "Anfangswertproblem" kommt hinzu, dass es recht mühsam ist, wenn die Berechnung in jedem Einzelfall speziell durchgefürt werden muss. Beide Schwierigkeiten lassen sich jedoch umgehen durch eine Tabellierung, resp. eine graphische Darstellung der Zusammenhänge. Für eine bestimmte Stahlsorte sind die benötigten Grundlagen, das heisst das Spannungs-Dehnungs-Diagramm $\varepsilon_{e}\left(\sigma_{e}\right)$ und das Verbundgesetz $\tau_{v}(v)$, zumindest für einen bestimmten Stabdurchmesser, weitgehend Invarianten und können durch entsprechende Versuche ermittelt werden. Es ist daher eine einmalige Berechnung, auch mit Hilfe einer elektronischen Rechenmaschine, und eine Darstellung zum Beispiel gemäss Bild 8 möglich.

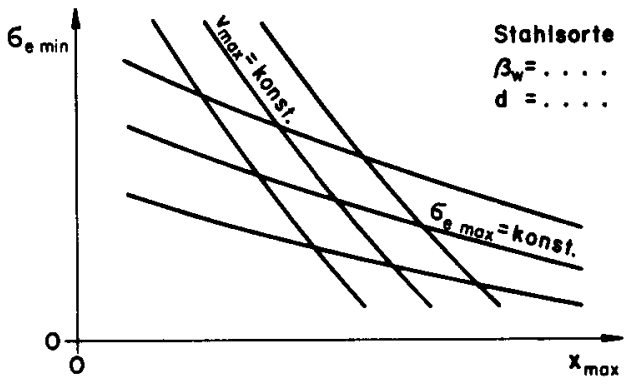

Bild 8

Mögliche Darstellung der $\mathrm{Zu}$ sammenhänge in der Zugzone eines Biegeriss-Elementes

Es werden noch die folgenden Bezeichnungen eingeführt (vergleiche Bild 7):

$$
\begin{aligned}
\varepsilon_{e 0} & =\varepsilon_{e \min } \\
\sigma_{e \min } & =\sigma_{e}\left(\varepsilon_{e \min }\right) \\
\sigma_{e 0} & =\sigma_{e \min } \\
\sigma_{e \max } & =\sigma_{e}\left(\varepsilon_{e \max }\right) \\
\Delta \sigma_{e \text { tot }} & =\sigma_{e \max }-\sigma_{e \min }
\end{aligned}
$$


Unter der Voraussetzung, dass bei $\varepsilon_{\text {emin }}$ stets $v=v_{0}=0$ ist, kann der Zusammenhang zwischen den Grössen

$$
G_{\text {emin }}, x_{\max }, G_{\text {emax }}, v_{\max }
$$

aufgetragen werden, wobei der Durchmesser $d$ und die Betonfestigkeit $\beta_{w}$ für jedes Diagramm als unveränderliche Grössen angenommen werden müssen. Anstelle der verwendeten Parameter könnte in der Darstellung zum Beispiel $\varepsilon_{\text {emax }}, \varepsilon_{\text {emin }}, \Delta \sigma_{e \text { tot }}$, und so weiter, benützt werden. Auch liesse sich der Verbundkoeffizient $\kappa$ als weitere abhängige Variable in ein solches Diagramm einbeziehen. Bild 8 zeigt generell eine der verschiedenen Darstellungsmöglichkeiten.

Zusammenfassend kann somit festgehalten werden:

Für eine bestimmte Stahlsorte, charakterisiert durch das Spannungs-DehnungsDiagramm und das Verbundgesetz, können Tabellen beziehungsweise Diagramme aufgestellt werden, die sämtliche Grössen enthalten, welche die Beanspruchungen und Verformungen in der Zugzone eines Biegeriss-Elementes beschreiben.

\section{b) Diagramm für Riegeltorstahl}

Im folgenden sollen die oben generell entwickelten Diagramme für Riegeltorstahl ausgearbeitet werden. Da die Versuchsbalken der Untersuchungen [7] und [8] mit dieser Stahlart armiert waren, ergeben sich interessante Kontrollund Vergleichsmöglichkeiten.

Die Aufstellung solcher Diagramme ist für Riegeltorstahl verhältnismässig einfach, da das Verbundgesetz mit genügender Genauigkeit als Parallele zur Abszisse (vergleiche Bild 6) angenommen werden kann:

$$
\tau_{\mathrm{v}}(\mathrm{v}) \cong \text { konst. }=\tau_{\mathrm{v}}^{*}
$$

Aufgrund verschiedener Untersuchungen, insbesondere von [12] und [13] , ist für liegende Einbettung etwa anzusetzen: 


$$
\begin{gathered}
-31- \\
\tau_{v}^{*}=(0,13 \div 0,18) \beta_{w}
\end{gathered}
$$

Die Integration von ( 17 ) ergibt:

$$
\Delta G_{\text {tot }}=G_{e \max }-G_{e \min }=\int_{G_{\text {min }}}^{G_{e \max }} d \sigma_{e}=\int_{0}^{s / 2} \tau_{v}(v) \frac{4}{d} d x=\tau_{v}^{*} \cdot \frac{4}{d} \cdot \frac{s}{2}
$$

Aus ( 20 ) wird:

$$
v_{\max }=\int_{0}^{v_{\max }} d v=\int_{0}^{s / 2} \varepsilon_{e}\left(\sigma_{e}\right) d x
$$

Nach Transformation von $d x$ erhält man:

$$
v_{\max }=\frac{w}{2}=\frac{d}{4 \tau_{v}{ }^{*}} \cdot \int_{\sigma_{\min }}^{\sigma_{e} \max } \varepsilon_{e}\left(\sigma_{e}\right) d \sigma_{e}
$$

Die maximale Verschiebung $v_{\max }$, respektive die halbe Rissweite $\mathrm{w}$, kann also abgesehen von einem konstanten Multiplikator - aus dem Spannungs-DehnungsDiagramm des Stahles durch Integration der Dehnungen über die Spannungen zwischen $\sigma_{\mathrm{e} \min }$ und $\sigma_{\mathrm{e} \max }$ berechnet werden.

Die Spannungs-Dehnungs-Diagramme des Riegeltorstahles verschiedenen Durchmessers weisen, was deren Form betrifft, nur geringe Unterschiede auf. In Bild 9 sind in normierter Darstellung die im Bericht [7] wiedergegebenen Diagramme aufgetragen. Es handelt sich um Stäbe mit den folgenden physikalischen Eigenschaften:

$$
\mathrm{d}=8,10,12,18 \mathrm{~mm}
$$




$$
\begin{aligned}
& E_{\mathrm{e}}=2,02 \cdot 10^{6} \div 2,12 \cdot 10^{6} \mathrm{~kg} / \mathrm{cm}^{2} \\
& G_{\mathrm{s}}=39,8 \div 46,8 \mathrm{~kg} / \mathrm{mm}^{2} \\
& \beta_{\mathrm{z}}=51,0 \div 58,0 \mathrm{~kg} / \mathrm{mm}^{2} \\
& \lambda_{\mathrm{Gl}}=5,2 \div 8,8 \%
\end{aligned}
$$

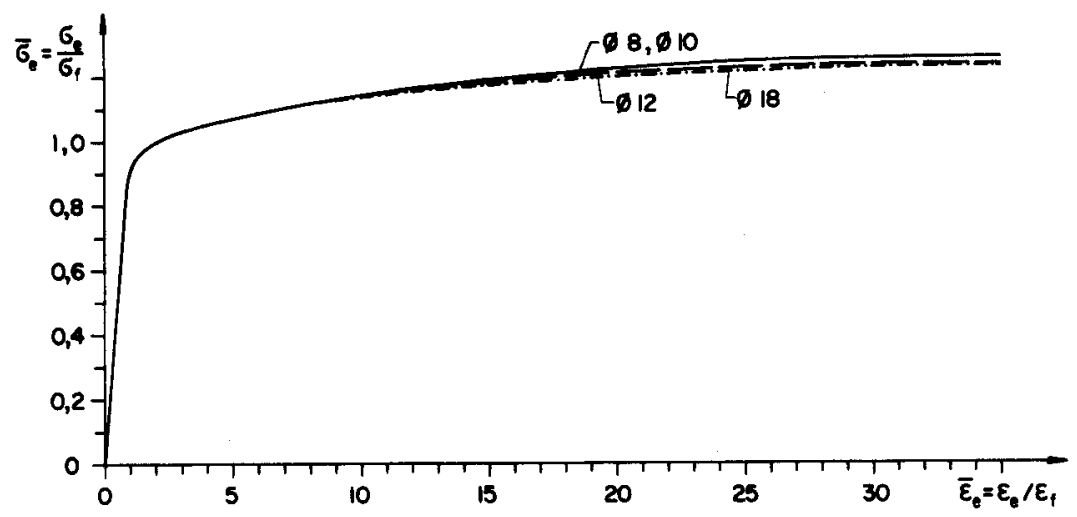

\section{Bild 9}

Spannungs-Dehnungs-Diagramme von Riegeltorstahl nach [7] in normierter Darstellung

Auf der Abszisse beziehungsweise Ordinate von Bild 9 sind die Grössen

$$
\bar{\varepsilon}_{e}=\frac{\varepsilon_{e}}{\varepsilon_{f}}, \quad \bar{\sigma}_{e}=\frac{\sigma_{e}}{\sigma_{f}}
$$

abgetragen. Die Vergleichsgrössen $\varepsilon_{f}$ und $G_{f}$ bedeuten die nominelle Fliessspannung beziehungsweise Fliessdehnung nach Bild 2. Man erkennt in Bild 9, dass die normierten Kurven sehr nahe beieinander liegen und als Näherung ein einziges normiertes Spannungs-Dehnungs-Diagramm $\bar{\varepsilon}_{\mathrm{e}}\left(\bar{\sigma}_{\mathrm{e}}\right)$ nach Bild 10 gewăhlt werden kann. 


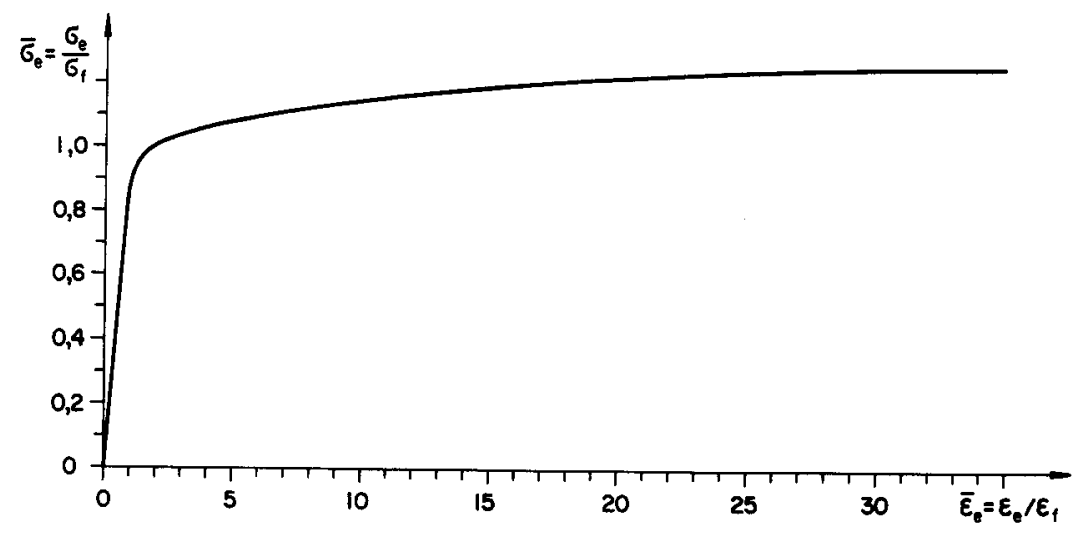

\begin{tabular}{|l|l|l|l|l|l|l|l|l|l|l|}
\hline $\bar{G}_{\mathrm{e}}$ & 0 & 0,5 & 0,8 & 0,9 & 0,96 & 1,00 & 1,033 & 1,064 & 1,089 & 1,117 \\
\hline $\bar{\varepsilon}_{\mathrm{e}}$ & 0 & 0,5 & 0,8 & 0,99 & 1,325 & 1,95 & 3,00 & $\mathbf{4 , 5 0}$ & $\mathbf{6 , 0 0}$ & $\mathbf{8 , 0 0}$ \\
\hline
\end{tabular}

\begin{tabular}{|c|c|c|c|c|c|c|c|c|c|c|}
\hline $\bar{G}_{\mathrm{e}}$ & 1,142 & 1,162 & 1,178 & 1,192 & 1,204 & 1,221 & 1,233 & 1,242 & 1,245 & 1,250 \\
\hline $\bar{\varepsilon}_{\mathrm{e}}$ & 10,0 & 12,0 & 14,0 & 16,0 & 18,0 & 21,0 & 24,0 & 27,0 & 30,0 & 35,0 \\
\hline
\end{tabular}

\section{Bild 10}

Normiertes Spannungs-Dehnungs-Diagramm für Riegeltorstahl

Somit lässt sich Gleichung ( 27 ) wie folgt anschreiben:

$$
v_{\max }=\frac{w}{2}=\frac{d}{4 \tau_{v}{ }^{*}} \cdot \varepsilon_{f} \cdot \sigma_{f} \cdot \int_{\sigma_{\text {min }}}^{\bar{\sigma}_{\text {max }}} \bar{\varepsilon}_{e}\left(\bar{\sigma}_{e}\right) d \sigma_{e}
$$

Darin bedeuten:

$$
\bar{\sigma}_{\mathrm{e} \max }=\frac{\sigma_{\mathrm{emax}}}{\sigma_{\mathrm{f}}} ; \quad \bar{\sigma}_{\mathrm{emin}}=\frac{\sigma_{\mathrm{e} \min }}{\sigma_{\mathrm{f}}}
$$


Mit den Zwischengrössen $\mathbf{r}$ und $\overline{\mathbf{r}}$,

$$
r=\varepsilon_{f} \cdot \sigma_{f} \cdot \int_{\sigma_{\text {min }}}^{\bar{\sigma}_{e \max }} \bar{\varepsilon}_{e}\left(\bar{\sigma}_{e}\right) d \bar{\sigma}_{e}=\varepsilon_{f} \cdot \sigma_{f} \cdot \bar{r},
$$

vereinfacht sich die Gleichung ( 29 ) auf

$$
v_{\max }=\frac{\mathrm{w}}{2}=\frac{\mathrm{d}}{4 \bar{\tau}_{\mathrm{v}}^{*}} \cdot \varepsilon_{\mathrm{f}} \cdot \sigma_{\mathrm{f}} \cdot \overline{\mathrm{r}}
$$

Die Integralfunktion $\overline{\mathbf{r}}$ wurde für das normierte Spannungs-Dehnungs-Diagramm nach Bild 10 für die variablen Grössen $\bar{\sigma}_{\mathrm{emax}}$ und

$$
\Delta \bar{\sigma}_{\text {etot }}=\frac{\Delta \sigma_{\text {etot }}}{\sigma_{\mathrm{f}}}
$$

berechnet und in Tafel 1 aufgetragen.

Aus derselben Darstellung ist auch die Variation des Verbundkoeffizienten $\kappa$ ersichtlich. Mit Gleichung ( 33 ) und

$$
\bar{\varepsilon}_{\text {emax }}=\frac{\varepsilon_{\text {e max }}}{\varepsilon_{f}}
$$

wird aus einer Kombination von ( 13 ), ( 26 ) und ( 32 ):

$$
\kappa=\frac{\bar{r}}{\Delta \bar{\sigma}_{\text {e tot }} \cdot \bar{\varepsilon}_{\text {e max }}}
$$

Damit ist für den Fall des Riegeltorstahles eine besonders einfache Darstellung der Zusammenhänge zwischen der Rissweite und den Beanspruchungen und übrigen Verformungen der Zugzone gefunden. Tafel 1 enthält sämtliche Abhängigkeiten, da die Grössen $\sigma_{f}=\sigma_{s}, \tau_{v}{ }^{*}$, , und s noch frei gewählt werden können. 


\subsection{Versagen der Zugarmierung}

Unter gewissen Voraussetzungen kann in einem Biegeriss-Element die Einschnïrung der Zugarmierung zu einer Reduktion des übertragbaren Momentes und zum Bruch führen.

Wird für dieses Versagen des Stahles der Beginn der Einschnürung als massgebend betrachtet und die entsprechende Stahldehnung mit $\varepsilon_{\mathrm{er}}$ bezeichnet, so heisst dies:

$$
\varepsilon_{\text {emax }}=\varepsilon_{\text {er }}
$$

Die kritische Rissweite infolge Versagen des Stahles beträgt damit nach ( 13 ):

$$
\mathrm{w}_{\text {krite }}=\kappa \cdot s \cdot \varepsilon_{\text {er }}
$$

Darin ist $\kappa$ nach dem im vorangehenden Abschnitt 2.21 entwickelten Verfahren, beziehungsweise mit den entsprechenden Tabellen oder Diagrammen, zu bestimmen. Der kritische Risswinkel ergibt sich nach ( 10 ):

$$
\omega_{\text {krite }}=\frac{\kappa \cdot s}{h-z} \cdot \varepsilon_{\text {er }}
$$

Darin ist $z$ entsprechend Gleichung ( 5 ) einzusetzen, wobei jedoch anstelle der nominellen Fliessspannung $\sigma_{f}$ die Bruchfestigkeit $\beta_{z}$ verwendet werden kann:

$$
z=\frac{h}{0,8 \cdot \beta} \cdot\left(\mu \cdot \beta_{z}-\mu \cdot \sigma_{f}^{\prime}\right)
$$

Die Dehnung in der Zugarmierung beim Beginn der Einschnürung beträgt:

$$
\varepsilon_{\text {er }}=\lambda_{G l}+\frac{\beta_{z}}{E_{e}}
$$


Darin bedeuten $\lambda_{G l}$ die ausserhalb der Einschnürungszone gemessene Gleichmassdehnung und $\beta_{z}$ die Bruchfestigkeit. Da der elastische Anteil $\beta_{z} / E_{e}$ gegenüber $\lambda_{\mathrm{Gl}}$ klein ist und ausserdem die Bestimmung von $\lambda_{\mathrm{Gl}}$ am Probestab einer gewissen Streuung unterworfen ist (Messgenauigkeit beschränkt), kann vereinfachend gesetzt werden:

$$
\varepsilon_{\mathrm{er}} \cong \lambda_{\mathrm{GI}}
$$

Das Erreichen der Gleichmassdehnung, das heisst der Beginn der Einschnürung und damit die Reduktion des übertragbaren Momentes, bedeutet bei einem statisch unbestimmten Tragwerk nicht notwendigerweise auch bereits den Zusammenbruch. Die Versuche [7] zeigten, dass infolge der Momentenumlagerung auf "stărkere" Stellen des Tragwerkes die Gesamtlast unter Umständen trotzdem noch ein wenig gesteigert werden kann. Im Sinne einer einfachen und sicheren Regelung wird mit (40) jedoch definiert, dass in einem Biegeriss-Element die kritische Rissweite beziehungsweise der kritische Risswinkel infolge Versagen der Zugarmierung dann vorhanden ist, wenn in derselben die Gleichmassdehnung erreicht worden ist.

\subsection{Verformungen in der Druckzone}

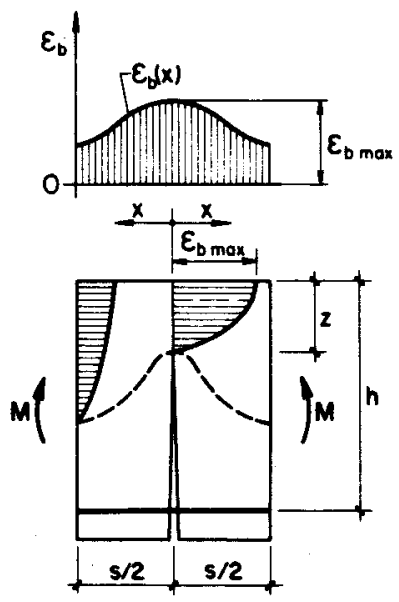

\section{Bild 11}

Bereich eines einzelnen Biegerisses ("Biegeriss-Element"); genereller Verlauf der Betonstauchungen in der Druckzone bei reiner Biegung 
Bild 11 zeigt generell den Verlauf der Betonstauchungen in der Druckzone eines Biegeriss-Elementes. Im Rissequerschnitt konzentrieren sich die Stauchungen auf den Bereich zwischen dem Rissende und der Druckkante, während sie sich zwischen den Rissen über einen höheren Bereich erstrecken. Entsprechend variiert die Stauchung der Randfaser. Sie erreicht über dem Rissende ein Maximum und verringert sich zwischen den Rissen.

Wird die gesamte Verkürzung der Randfaser innerhalb des Biegeriss-Elementes mit w' bezeichnet, so kann analog zu ( 13 ) angeschrieben werden:

$$
w^{\prime}=2 \cdot \int_{0}^{s / 2} \varepsilon_{b}(x) \cdot d x=k^{\prime} \cdot s \cdot \varepsilon_{b \max }
$$

Darin charakterisiert $\kappa^{\prime}$ die Variation der Betonrandstauchung. $\kappa^{\prime}$ ist gleich dem Verhältnis der in Bild 11 vertikal schraffierten Fläche zur Fläche des umschriebenen Rechteckes, oder auch gleich dem Quotienten der mittleren Betonstauchung

$$
\varepsilon_{\mathrm{bm}}=\frac{\mathrm{w}^{\prime}}{\mathrm{s}}
$$

und der maximalen Betonstauchung $\varepsilon_{\mathrm{b} \text { max }}$ :

$$
\kappa^{\prime}=\frac{2 \int_{0}^{s / 2} \varepsilon_{b}(x) d x}{s \cdot \varepsilon_{b \max }}=\frac{\varepsilon_{b m}}{\varepsilon_{b \max }}
$$

Dem Verfasser sind keine experimentellen Untersuchungen über den Verlauf von $\varepsilon_{b}(x)$ bekannt. Als wesentlichste Parameter können jedoch die geometrischen Grössen $z$ und $s$ bezeichnet werden. Die Variation der Betonstauchungen ist umso grösser, das heisst der Wert für $\kappa^{\prime}$ ist umso kleiner, je kleiner der Abstand $z$ des Rissendes vom Druckrand im Verhältnis zum Rissabstand $s$ ist. Für $\kappa$ ' werden daher die folgenden Ansätze gewählt: 


$$
\begin{aligned}
& \frac{z}{s}=1,0: \kappa^{\prime}=\left(\frac{z}{s}\right)^{k^{\prime}}, \text { mit } 0 \leq k^{\prime} \leq 1,0 \\
& \frac{z}{s} \geq 1,0: \kappa^{\prime}=1,0
\end{aligned}
$$

Damit ergibt sich die maximale Betonstauchung in Abhängigkeit von der Verkürzung der Randfaser:

$$
\begin{aligned}
& \frac{z}{s} \leq 1,0: \varepsilon_{b \max }=\frac{w^{\prime}}{s\left(\frac{z}{s}\right)^{k^{\prime}}} \\
& \frac{z}{s}=1,0: \varepsilon_{b \max }=\frac{w^{\prime}}{s}
\end{aligned}
$$

Mit Gleichung ( 45 ) beziehungsweise ( 47 ) wird angenommen, dass für $\mathrm{z}>\mathrm{s}$ keine Variation der Randdehnung mehr auftrete. Tatsächlich ist in diesem Fall das Rissende im Verhältnis zum Rissabstand sehr weit entfernt vom Druckrand, sodass die gedrückte Fläche an den seitlichen Rändern des Biegeriss-Elementes nur unwesentlich grösser als in den Rissequerschnitten ist. Die Veränderlichkeit von $\varepsilon_{b}(x)$ wird dadurch vernachlässigbar klein.

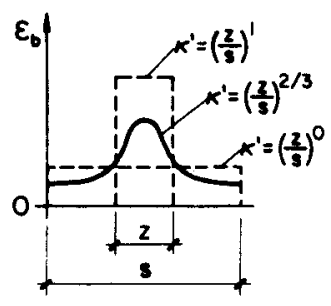

\section{Bild 12}

Verlauf der Betonrandstauchungen bei verschiedenen Ansätzen für $\mathrm{k}^{\prime}(\mathrm{z} / \mathrm{s}=0,3)$

Schwieriger ist es, eine Annahme für den Exponenten $k^{\prime}$ in Gleichung ( 44 ) beziehungsweise ( 46 ) zu treffen. Würde $\mathbf{k}^{\prime}=0$ gesetzt, so verschwände auch im ganzen Bereich $0 \leqslant z \leqslant s$ die Veränderlichkeit von $\varepsilon_{b}(x)$. Diese Annahme 
würde der Hypothese vom Ebenbleiben der Querschnitte entsprechen, welche hier jedoch offensichtlich nicht zutreffen kann. Anderseits wïrde $k^{\prime}=1$ bedeuten, dass die maximale Betonstauchung sich $\mathrm{zu}$

$$
\varepsilon_{\mathrm{b} \max }=\frac{\mathrm{w}^{\prime}}{\mathrm{z}}
$$

ergäbe, was einer Konzentration der Stauchungen über dem Riss auf eine Breite $z$ gleichkäme. Für $z \leqslant s$ wäre demnach die maximale Betonstauchung $\varepsilon_{b \text { max }}$ unabhängig vom Rissabstand. In Bild 12 sind für $z / s=0,3$ die den Werten $k^{\prime}=$ 0 und $k^{\prime}=1$ entsprechenden Rechtecke gestrichelt dargestellt. In Bild 13 ist das Verhältnis der maximalen Randstauchung $\varepsilon_{\mathrm{b} \text { max }}$ zur mittleren Stauchung $\varepsilon_{\mathrm{bm}}$, das heisst der reziproke Wert von $\kappa^{\prime}$, in Abhängigkeit vom Quotienten $z / s$ für $\mathbf{k}^{\prime}=0$ und $\mathbf{k}^{\prime}=1$ ebenfalls gestrichelt eingetragen.

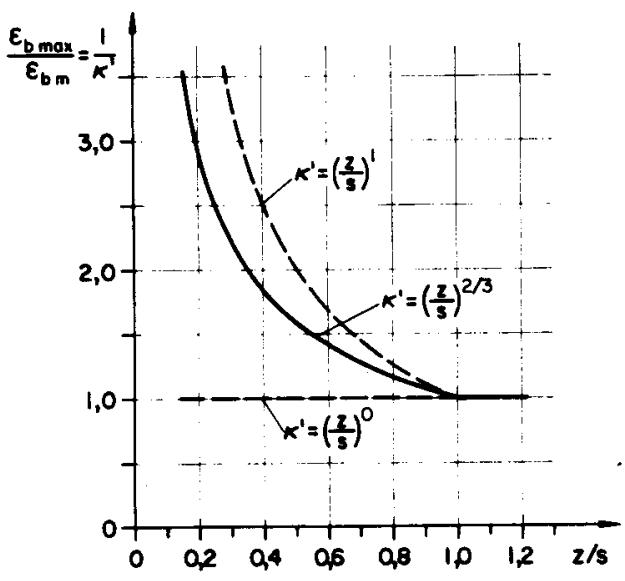

\section{Bild 13}

Verhältnis der maximalen zur mittleren Betonrandstauchung in Abhängigkeit von $z / s$ und bei verschiedenen Ansätzen für $\kappa^{\prime}$

Aus beiden Bildern erkennt man, dass die Werte $k^{\prime}=1$ und $k^{\prime}=0$ offensichtlich Grenzwerte der Variationen $\varepsilon_{b}(x)$ darstellen, die in Wirklichkeit nicht vorkommen können.

Aufgrund verschiedener Ueberlegungen und Beobachtungen, insbesondere bei den 
Versuchen [7] und [8], muss $k^{\prime}$ relativ gross angesetzt werden. Dafür sprechen insbesondere die folgenden Gründe:

1. Die ersten Zerstörungen in der Biegedruckzone treten auch bei grösseren Verhältnissen $\mathrm{z} / \mathrm{s}$ meist über einem Rissende auf. Es muss daher dort eine verhăltnismässig starke Konzentration der Betonstauchungen vorhanden sein.

2. Da die maximale Betonstauchung für das Versagen der Biegedruckzone als massgebend angesehen wird (vergleiche 2.24), interessiert der Verlauf von $\varepsilon_{b}(x)$ beziehungsweise die relative Grösse von $\varepsilon_{b \max }$ nur unmittelbar vor dem Bruch. Diesem Zustand entsprechen normalerweise bedeutende Verformungen des Biegeriss-Elementes. Weil dabei die Randfaser erhebliche plastische Deformationen erleidet, konzentrieren sich mit grösser werdenden Verformungen die Betonstauchungen stärker auf den Bereich des Rissequerschnittes.

3. Die Annahme eines eher "hohen" Wertes für k' liegt bezüglich der Berechnung des kritischen Gelenkwinkels infolge Versagen der Biegedruckzone auf der sicheren Seite (vergleiche 2.24).

Mit

$$
\mathbf{k}^{\prime}=\frac{2}{3}
$$

lauten die Gleichungen ( 44 ) und ( 46 ):

$$
\begin{array}{ll}
\frac{z}{s} \leqslant 1,0: & \kappa^{\prime}=\left(\frac{z}{s}\right)^{2 / 3} \\
\frac{z}{s}<1,0: & \varepsilon_{b \max }=\frac{w^{\prime}}{s\left(\frac{z}{s}\right)^{2 / 3}}
\end{array}
$$

In den Bildern 12 und 13 sind die entsprechenden Kurven eingetragen. Bild 14 zeigt die ungefähre Verteilung der Randstauchungen in Abhängigkeit vom Verhältnis $\mathrm{z} / \mathrm{s}$ (Minimalwerte geschätzt). In diesem Bild wurde bei gleichbleibendem Rissabstand $s$ eine für verschiedene Grössen $z$ stets gleiche Verkürzung der Randfaser vorausgesetzt, sodass die Flächen unter den glockenförmigen Kurven übereinstimmen. 


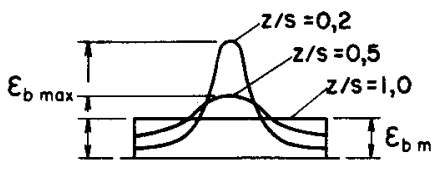

\section{Bild 14}

Verlauf der Randstauchungen in Abhängigkeit von $\mathrm{z} / \mathrm{s}$ für

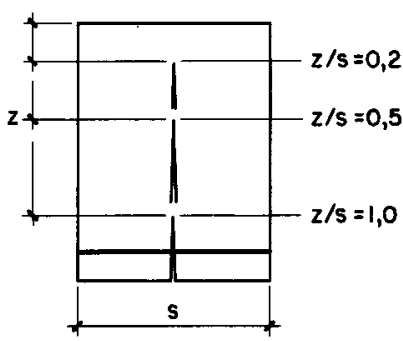

Für die Berechnung der Verkürzung $w^{\prime}$ der Randfaser von der Länge $s$ wird noch die folgende Verträglichkeitsbedingung eingeführt:

$$
\mathbf{w}^{\prime}=\omega \cdot \mathrm{z}=\mathrm{w} \cdot \frac{\mathrm{z}}{\mathrm{h}-\mathrm{z}}
$$

Es wird somit angenommen, dass $w^{\prime}$ proportional zum Risswinkel $\omega$ und zum Abstand $z$ des Rissendes von der Druckkante ist. Damit ergibt sich die maximale Betonstauchung wie folgt:

$$
\begin{aligned}
& \frac{z}{s} \leq 1,0: \quad \varepsilon_{b \max }=\frac{w}{s} \cdot \frac{z}{h-z} \cdot \frac{1}{\left(\frac{z}{s}\right)^{2 / 3}} \\
& \frac{z}{s}=1,0: \quad \varepsilon_{b \max }=\frac{w}{s} \cdot \frac{z}{h-z}
\end{aligned}
$$




\subsection{Versagen des Betons}

Eine häufige Ursache für den Bruch in einem Biegeriss-Element ist die Zerstörung der Biegedruckzone. Wird für das Versagen des Betons die maximale Randstauchung als massgebend betrachtet, so gilt:

$$
\varepsilon_{\mathrm{b} \max }=\varepsilon_{\mathrm{br}}
$$

Darin ist $\varepsilon_{\mathrm{br}}$ die Betonbruchstauchung. Die kritische Rissweite beim Versagen des Betons ergibt sich mit ( 51 ) in ( 49) und (50) wie folgt:

$$
\begin{aligned}
& \frac{z}{s} \leqslant 1,0: w_{k r i t b}=\varepsilon_{b r} \cdot s \cdot \frac{h-z}{z} \cdot\left(\frac{z}{s}\right)^{2 / 3} \\
& \frac{z}{s}+1,0: w_{k r i t b}=\varepsilon_{b r} \cdot s \cdot \frac{h-z}{z}
\end{aligned}
$$

Entsprechend wird der kritische Risswinkel beim Versagen des Betons:

$$
\begin{aligned}
& \frac{z}{s} \leq 1,0: \omega_{\text {kritb }}=\varepsilon_{b r} \cdot \frac{s}{z} \cdot\left(\frac{z}{s}\right)^{2 / 3} \\
& \frac{z}{s}=1,0: \omega_{\text {krit b }}=\varepsilon_{b r} \cdot \frac{s}{z}
\end{aligned}
$$

Bügel und damit im Zusammenhang auch Druckstäbe bewirken gewissermassen eine Umschnürung der Biegedruckzone. Dadurch wird die Bruchstauchung $\varepsilon_{b r}$ gegenüber derjenigen in einer unarmierten Biegedruckzone oft wesentlich erhöht. Bei derart armierten Druckzonen kann es zudem vorkommen, dass die Zerstörung der Randfaser nicht einen sofortigen Bruch bewirkt. Vielmehr wird nur der Beton ausserhalb der Armierung gestaucht beziehungsweise langsam abgelöst und die entsprechende Druckkraft ganz oder teilweise durch den umschnürten Beton und eventuell die Druckarmierung zusätzlich übernommen. Daher ist in solchen Fällen der Beginn der Stauchung oft schwierig festzustellen. Nach deut- 
lichen Stauchungserscheinungen zeigt sich meist eine stetige Reduktion des übertragbaren Biegemomentes, analog wie nach Beginn der Einschnürung der Zugarmierung (vergleiche 2.22). Bei starker Umschnürung kann unter Umständen ein gegenüber vor dem Stauchen des Betons nur verhältnismässig gering reduziertes Moment weiterhin, das heisst bis zu recht ansehnlichen Deformationen, übertragen werden. Auch für diese letztere, in Biegebereichen zwar seltenere Art von Biegedruckzonen wird mit ( 51 ) als einfache Regel angenommen, dass in einem Biegeriss-Element die kritische Rissweite beziehungsweise der kritische Risswinkel dann vorhanden ist, wenn die nach (49) beziehungsweise ( 50 ) berechnete Randstauchung $\varepsilon_{\mathrm{b} \max }$ gleich der Bruchstauchung $\varepsilon_{\mathrm{b} r}$ geworden ist. Bei der Festlegung von $\varepsilon_{b r}$ muss die Umschnürungswirkung berücksichtigt werden.

Die Grösse von $\varepsilon_{b \mathrm{r}}$ hängt somit von verschiedenen Einflussgrössen ab. Deren wichtigste sind:

- Betonqualität

- Querarmierung (Bügel, eventuell Spiralen)

- Längsarmierung (Druckstäbe)

Für gut umschnürte Biegedruckzonen wird zum Beispiel in [14] eine Bruchstauchung in plastischen Gelenken von 10 bis $12 \%$ angegeben. Aehnliche oder noch höhere Werte können durch die Anordnung von eigentlichen Spiralarmierungen, wie sie sonst bei Stützen gebräuchlich sind, erzielt werden [15]. Da es sich im letzteren Fall jedoch um eine spezielle Massnahme handelt, soll hier nur auf die Wirkung von Bügeln und Druckstäben näher eingegangen werden.

H. Rüsch und S. Stöckl haben durch systematische Versuche an exzentrisch gedrückten Prismen die Einflüsse der oben angeführten Parameter auf das Verhalten der Biegedruckzone untersucht [16]. Erwartungsgemäss wurde festgestellt, dass die Umschnürungswirkung von Bügeln ausser vom Gesamtquerschnitt derselben auch von der Feinheit ihrer Aufteilung abhängt. Druckstäbe können die Wirkung der Bügel noch verbessern. Um den Einfluss der Bügel zu erfassen, wurden die folgenden Definitionen eingefuihrt: 
Querarmierungsgehalt: $\quad \mu_{q}=\frac{F_{q}}{t \cdot c}$

Aufteilungsgrad:

$$
A=\left[\frac{c-t / 2}{c}\right]^{2}
$$

Die Bedeutung der Grössen $F_{q}$, $t$ und $c$ geht aus Bild 15 hervor.

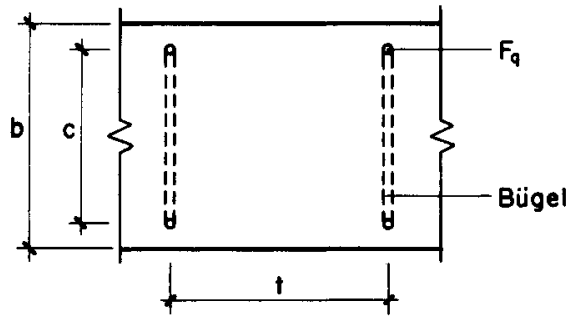

Bild 15

Definition von Querarmierungsgehalt und Aufteilungsgrad in der Biegedruckzone

Die Versuche zeigten, dass sich die Bruchstauchung infolge der Umschnürungswirkung ungefähr linear mit $\mu_{q}$ und $A$ erhöht. Für hochfesten Beton ist die $\mathrm{Zu}-$ nahme jedoch wesentlich geringer als für Beton niedriger Festigkeit, wobei sich entsprechend dem verschiedenartigen Formänderungsverhalten auch für unarmierten Beton $\left(\mu_{q}=A=0\right)$ deutliche Unterschiede ergaben. Die Ergebnisse der Untersuchungen [16] können, was die Wirkung der Betonfestigkeit und der Bügel anbelangt, durch die folgende Formel erfasst werden:

$$
\varepsilon_{b r}=\varepsilon_{b r 0} \cdot\left[\frac{\beta_{w 0}}{\beta_{w}}\right]^{1 / 3}+1,4 \cdot \mu_{q} \cdot A \cdot\left[\frac{\beta_{w 0}}{\beta_{w}}\right]
$$


Darin sind $\beta_{w 0}=250 \mathrm{~kg} / \mathrm{cm}^{2}, \varepsilon_{b r 0}=0,004$ sowie $\beta_{w}$ in $\mathrm{kg} / \mathrm{cm}^{2}$ einzusetzen. Der erste Summand bedeutet die Bruchstauchung in einer unbewehrten Biegedruckzone. Der zweite erfasst den Einfluss des Querarmierungsgehaltes und des Aufteilungsgrades. Die Formel ( 58 ) ist so gestaltet, dass die resultierenden Werte mit den Versuchsergebnissen nach [16] zusammenfallen oder darunter liegen. Die Abweichungen sind im allgemeinen gering und betragen maximal $20 \%$ der gemittelten Versuchswerte. In Bild 16 sind die Zusammenhänge graphisch dargestellt.

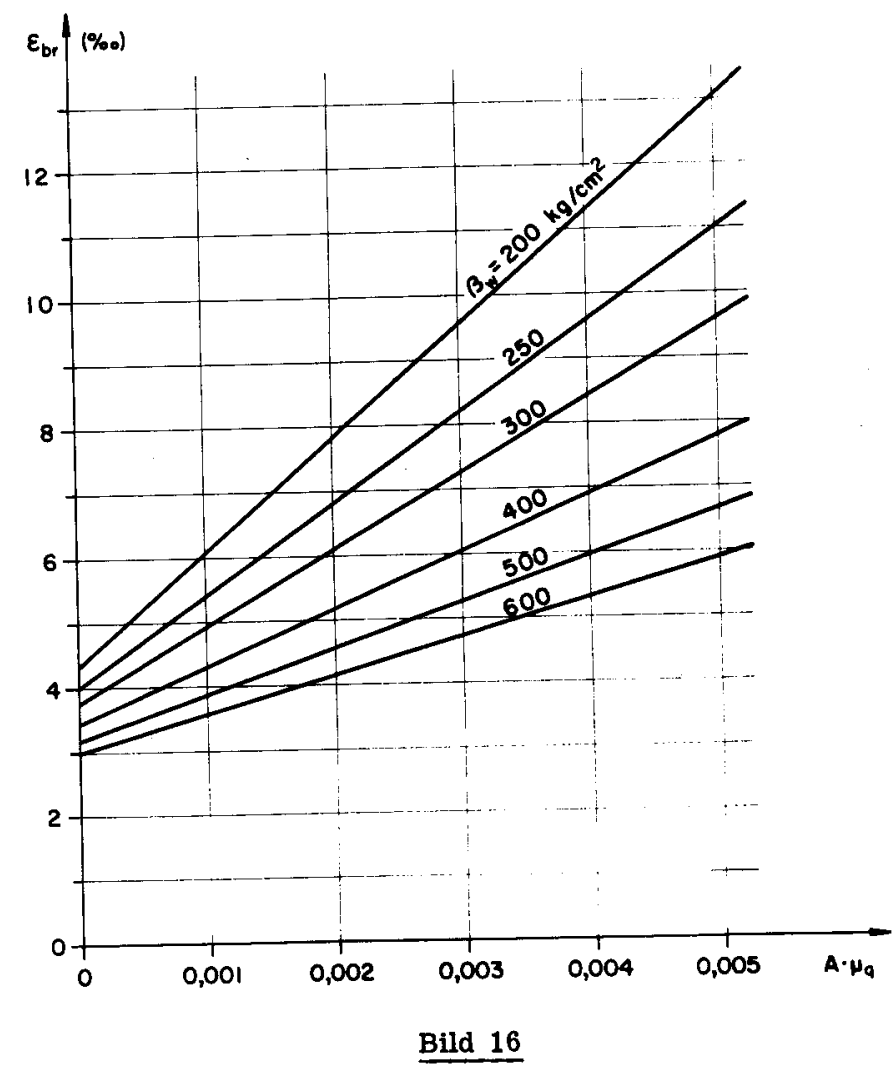

Abhängigkeit der Bruchstauchung am Rand der Biegedruckzone vom Querarmierungsgehalt und Aufteilungsgrad der Bügel 
Druckstäbe vermindern das Hervorquellen des Betons zwischen den Bügeln. Dies ist vor allem bei Beton höherer Festigkeit der Fall. Die Erhöhung der Randstauchung beim Bruch betrug nach [16] für $\beta_{\mathrm{w}}=600 \mathrm{~kg} / \mathrm{cm}^{2}$ bei sehr starker Druckarmierung etwas mehr als $1 \% 0$. Für $\beta_{\mathrm{w}}=300 \mathrm{~kg} / \mathrm{cm}^{2}$ hingegen war die Wirkung von Druckstäben nur geringfügig. Der Einfluss von Druckstäben auf die Bruchstauchung von Biegedruckzonen ist daher in ( 58 ) nicht berücksichtigt worden.

Es muss hier noch ausdrücklich betont werden, dass ( 58 ) nur für Biegedruckzonen gilt, bei denen tatsächlich von einer Umschnürung durch Bügel gesprochen werden kann. Diese Voraussetzung trifft vor allem für Balken mit rechteckigem Querschnitt zu oder für solche mit zur Stegdicke relativ schmalen Flanschen, welche durch "Flanschbügel" umfasst werden. Bei breiteren Flanschen sollte vorsichtigerweise nur der erste Summand von ( 58 ) in Rechnung gestellt werden, sofern nicht entsprechende Versuchsresultate vorliegen.

\section{3. Rissabstand}

Bei den vorangegangenen Untersuchungen und Herleitungen wurde der Rissabstand als bekannt vorausgesetzt. Zweck dieses Abschnittes ist es, Regeln für die Berechnung desselben anzugeben.

Ueber die Rissbildung in Stahlbetonbalken existieren zahlreiche Abhandlungen (zum Beispiel [17] , [18] , [19]). Die entwickelten Formeln laufen jedoch fast alle auf die Berechnung einer voraussichtlichen Rissweite im Zusammenhang mit bestimmten mittleren Dehnungen in der Zugzone hinaus. Diese Dehnungen entsprechen meist Beanspruchungen der Zugarmierung im Gebrauchszustand, jedenfalls solchen im Bereich elastischer Stahlbeanspruchungen $\left(\sigma_{e} \leqslant \sigma_{p}\right)$.

Für unsere Zwecke hingegen interessiert einzig und allein der Abstand der "Hauptrisse" (vergleiche 2.11) beim Erreichen des kritischen Gelenkwinkels, das heisst wenn die Zugarmierung normalerweise bereits plastische Deformationen erlitten hat. Dabei stellt sich die Frage, wie weit der Rissabstand von der Beanspruchung beziehungsweise dem Verformungszustand der Stahleinlagen abhängt. Oder mit 
anderen Worten: Ist zu erwarten, dass zu den Rissen hinzu, welche im Bereich elastischer Stahlbeanspruchungen vorhanden sind, im Laufe der plastischen Rotation noch weitere Risse entstehen?

Die grundlegende Beziehung für die Berechnung des Rissabstandes ist die bekannte Gleichgewichtsbedingung (zum Beispiel [12] ):

$$
\int_{0}^{s} \tau_{v}(v) \cdot \sum u \cdot d x=\int_{0}^{\mathbf{F}} \sigma_{b} \cdot d F_{z}
$$

Darin ist $u$ der Stabumfang $\pi d, \sigma_{b}$ die Zugspannung im Beton und $F_{z}$ die Betonzugfläche. $\tau_{v}(v)$ ist die an einem Element der Staboberfläche von der Länge $d x$ wirkende Verbundspannung. Da diese grundsätzlich eine Funktion der Relativverschiebung $v$ des Stabes gegenüber dem Beton ist (vergleiche 2.21), wird die Zugspannung im Beton auch während der plastischen Rotation laufend verändert, sodass stets noch neue Risse entstehen können. Dies wird insbesondere bei ausgesprochenen Rippenstählen mit stark gekrümmtem Verbundgesetz (vergleiche Bild 6) vorkommen. Bei Rundstählen oder beim Riegeltorstahl hingegen, wo $\tau_{v}(v)$ bald einen konstanten Wert annimmt, sind gegenüber dem Rissebild im elastischen Beanspruchungsbereich im allgemeinen keine oder nur noch wenige zusätzliche Risse zu erwarten. Die Betonzugfläche $F_{z}$ kann in Biegeträgern meist nicht genau erfasst werden. Wir legen daher fest:

Rechteckquerschnitt:

$$
\begin{aligned}
& F_{z}=b h \\
& F_{z}=b_{0} h
\end{aligned}
$$

T- und I-Querschnitt

Ferner wird die Abhängigkeit der Verbundspannungen von der Relativverschiebung auch bei Rippenstählen vernachlässigt und die Verbundspannung als mittlerer Wert konstant angenommen:

$$
\tau_{\mathbf{v}}(\mathbf{v})=\tau_{\mathbf{v R}}
$$


Setzt man zudem die Betonzugspannung $\sigma_{b}$ beim Entstehen des Risses über die ganze Zugfläche gleich der Betonzugfestigkeit $G_{b z}$, so ergibt sich mit dem Armierungsgehalt der Zugfläche

$$
\mu_{z}=\frac{F_{e}}{F_{z}}=\frac{b}{b} \cdot \mu
$$

der Rissabstand aus ( 59 ) wie folgt:

$$
\mathbf{s}=\mathbf{k}_{\mathbf{R}} \cdot \frac{\mathrm{d}}{\boldsymbol{\mu}_{\mathrm{z}}} \cdot \frac{\sigma_{\mathrm{bz}}}{\tau_{v R}}
$$

Der Vergleich mit Versuchsresultaten (zum Beispiel [8], [13] ) hat ergeben, dass $k_{R} \cong 0,20$ gesetzt werden kann, also

$$
\mathbf{s} \cong 0,2 \cdot \frac{d}{\mu} \cdot \frac{\sigma_{b z}}{\tau_{v R}} \cdot \frac{b_{0}}{b},
$$

wenn für die Grössen $\sigma_{b z}$ und $\tau_{v R}$ etwa die folgenden mittleren Werte angenommen werden:

Betonzugfestigkeit:

$$
\sigma_{b z}=(1,2+1,5) \cdot \sqrt{\beta_{w}}
$$

Verbundspannung bei liegender Einbettung:

Rundstathle:

Riegeltorstahl:

Rippenstăhle:

$$
\begin{aligned}
& \tau_{\text {vR }}=(0,02 \div 0,08) \cdot \beta_{w} \\
& \tau_{v R}=(0,13 \div 0,18) \cdot \beta_{w} \\
& \tau_{v R}=(0,16 \div 0,24) \cdot \beta_{w}
\end{aligned}
$$

Der Unterschied zwischen den Werten $\tau_{v R}$ von Riegeltorstahl und Rippenstählen ist geringer als der Unterschied zwischen den maximalen Werten $\tau_{v}(v)$ in den entsprechenden Verbundgesetzen. Denn sofern auch die Armierung streckenweise 
erhebliche plastische Dehnungen aufweist und grössere Verschiebungen $v$ auftreten, ist beim Rippenstahl der Maximalwert von $\tau_{v}(v)$ nie über den ganzen Bereich $\mathrm{s} / 2$ vorhanden. Beim Riegeltorstahl hingegen wird dieser Wert schon nach relativ kleinen Verschiebungen erreicht, sodass $\tau_{v R}$ etwa dem Wert $\tau_{v}{ }^{*}$ entspricht (vergleiche 2.21).

Die Variation der Verbundspannungen $\tau_{\mathrm{vR}}$ innerhalb der einzelnen Stahlarten ist besonders bei Rundstählen gross, wo glatte oder schwach vernarbte Stäbe sich wesentlich anders als stark angerostete Stäbe verhalten. Vor allem bei Rundstahl und in vermindertem Masse bei den profilierten Stăhlen hat auch der Abstand der Stäbe vom Schalungsboden einen Einfluss, da durch Setzungen des Frischbetons an der Stabunterseite gerne Poren oder sogar Hohlräume entstehen. Die Regeln ( 65 ) für $\tau_{v R}$ dürften ausgesprochene Extremfälle nicht erfassen, im allgemeinen jedoch recht brauchbare Rechenwerte liefern.

Die Regeln ( 60 ) bis ( 65 ) gelten etwa für Armierungsgehalte $\mu_{z} \geq 0,7 \%$. Bei kleineren Armierungsgehalten sind nach den Erfahrungen des Verfassers die Rissabstände oft geringer als sie sich nach $(60)$ bis ( 65$)$ ergeben. Dies ist vermutlich unter anderem darauf zurückzuführen, dass die Eigenspannungen infolge unterschiedlichem Schwinden des Oberflăchen- und des Kernbetons verhältnismässig gross sind gegenüber den Wirkungen der oben eingeführten Parameter. Auch sind die systematischen Einflüsse der Grössen $\mu_{z}$ und d bei kleinen Armierungsgehalten verhältnismässig gering gegenüber den Streuungen der Festigkeitsgrössen $\sigma_{\mathrm{bz}}$ und $\tau_{\mathrm{vR}}$. Ausserdem entstehen erste Anrisse oft bei den Bügeln, da diese den Querschnitt der Betonzugfläche reduzieren und dadurch gewissermassen eine Kerbwirkung entsteht. Somit muss für kleinere Armierungsgehalte anstelle von (63a) in Betracht gezogen werden:

$$
\mathbf{s}=\mathbf{t}
$$

Aus den obigen Darlegungen geht hervor, dass der Rissabstand in Stahlbetonbalken verhältnismässig unsicher vorhergesagt werden kann. Anderseits wird in 2.51 noch gezeigt werden, dass dieser von wesentlicher Bedeutung für die Grösse des kritischen Gelenkwinkels sein kann. Es emphiehlt sich daher, eine praktische Berechnung des kritischen Gelenkwinkels stets für 2 deutlich verschiedene Rissabstände vorzunehmen. 
2.4. Kritischer Gelenkwinke1

Nachdem im vorangehenden Abschnitt 2.2 die Verformungen und der Bruch in einem einzelnen Biegeriss-Element behandelt wurten, soll nun wieder das ganze plastische Gelenk betrachtet werden.

\subsection{Allgemeine Berechnung}

Der "kritische Gelenkwinkel" ist dann vorhanden, wenn in einem Biegeriss des Gelenkes Versagen des Betons oder des Stahles eintritt. Unter den bisher getroffenen Voraussetzungen wird dies im breitesten Riss der Fall sein. Dieser befindet sich, da das Biegemoment längs des Gelenkes praktisch immer veränderlich ist, an der Stelle des maximalen Momentes. Ausser diesem "kritischen Riss" leisten auch die andern, schmäleren Risse einen Beitrag an den kritischen Gelenkwinkel. Dieser muss somit, ausgehend vom kritischen Riss, durch Berechnung und Addition der Rissweiten beziehungsweise Risswinkel der übrigen Risse des Gelenkes ermittelt werden. Ist im Gelenk nach Bild 1 die kritische Rissweite zum Beispiel im Riss Nr. 1 vorhanden, so ergibt sich der kritische Gelenkwinkel nach ( 9 ) mit den entsprechenden Definitionen "kritische Summe der Rissweiten" und "kritische Summe der Risswinkel" wie folgt:

$$
\theta_{k r}=\frac{k r i t \sum_{i}^{n}}{h-z}=\operatorname{krit} \sum_{1}^{n} \omega_{i}
$$

Zur Berechnung der einzelnen Rissweiten werden die in den vorangehenden $\mathrm{Ab}$ schnitten bereitgestellten Grundlagen verwendet.

\section{a) Praktisches Vorgehen}

Das praktische Vorgehen bei der Berechnung der Rissweiten beziehungsweise Risswinkel ist im allgemeinen folgendes: 
1. Es sind im kritischen Riss sowohl $w_{k r e}$ aus ( 37 ) als auch $w_{k r b}$ nach (52) beziehungsweise ( 53 ) zu berechnen. Massgebend ist der kleinere Wert.

2. Bei Versagen des Stahles gilt im kritischen Riss für die Stahldehnung nach (36) und ( 40$) \varepsilon_{\text {e max }}=\varepsilon_{e r} \cong \lambda_{G l}$, sowie $\sigma_{e \max }=\beta_{z^{\prime}}$. Ist das Versagen des Betons massgebend, so muss das zugehörige $\varepsilon_{\text {emax }}$ beziehungsweise $\sigma_{\text {emax }}$ mittels des unter 2.21 entwickelten Verfahrens beziehungsweise mit Hilfe entsprechender Tabellen oder Diagramme (zum Beispiel Bild 8) ermittelt werden.

3. Das Moment im kritischen Riss, welches gleich dem maximalen Moment im Gelenk ist, beträgt:

$$
M_{k r}=F_{e} \cdot y \cdot G_{e \max }
$$

Hiermit kann der Momentenverlauf im Bereiche des Gelenkes bestimmt werden.

4. In den Rissen neben dem kritischen Riss ergibt sich die maximale Stahlspannung aus

$$
G_{e \max i}=\frac{M_{i}}{F_{e}^{\cdot y}} .
$$

Mit der Differenz der Zugkräfte in zwei benachbarten Rissen,

$$
\Delta \mathrm{Z}_{\mathbf{i}, \mathbf{i}+1}=\mathrm{z}_{\mathbf{i}}-\mathrm{Z}_{\mathbf{i}+1}
$$

gilt auch der folgende Zusammenhang:

$$
G_{e \max i+1}=\sigma_{e \max i}-\frac{\Delta Z_{i, i+1}}{F_{e}}
$$

Ist die Querkraft neben dem kritischen Riss konstant, so kann gesetzt werden: 


$$
\Delta z_{i, i+1}=\frac{Q \cdot s}{y}=\text { konst. }=\Delta z
$$

Damit sind aus dem Spannungs-Dehnungs-Diagramm die Dehnungen $\varepsilon_{\text {emaxi }}$ ebenfalls bekannt. Es müssen gemäss ( 2 ) diejenigen Risse berücksichtigt werden, in denen $\varepsilon_{\text {emax } i} \geqslant \varepsilon_{f}$ ist.

5. Aus $\sigma_{\text {emaxi }}$ beziehungsweise $\varepsilon_{\text {emax } i}$ in den einzelnen Rissen werden nach 2.21 die zugehörigen Rissweiten bestimmt, worauf sich nach (67) der kritische Gelenkwinkel ergibt.

Im Abschnitt 2.21 war für die Herleitung der soeben verwendeten Beziehung zwischen Rissweite und Spannungen respektive Dehnungen der Zugarmierung angenommen worden, dass das einzelne Biegeriss-Element ausschliesslich durch ein längs des Elementes konstantes Biegemoment beansprucht werde. Der Einfluss einer Veränderlichkeit des Biegemomentes beziehungsweise derjenige der Querkraft auf den Verlauf der Verbundspannungen, Verschiebungen und Dehnungen wird beim beschriebenen Vorgehen vernachlässigt. Tatsächlich ist dieser Einfluss auf die resultierenden Rissweiten meist gering. Trotzdem sollen hier noch die allgemeinen Zusammenhänge dargestellt beziehungsweise ein verfeinertes Verfahren entwickelt werden.

\section{b) Verfeinertes Verfahren}

In Bild 17 ist der Bereich zwischen zwei benachbarten Biegerissen dargestellt. Dieser wird durch ein veränderliches Biegemoment und durch eine Querkraft beansprucht. Darunter ist generell der Verlauf der Stahldehnungen sowie der Verbundspannungen aufgetragen.

Das veränderliche Biegemoment, beziehungsweise die Querkraft, haben eine unterschiedliche Zugkraft $\mathrm{Z}$ in der Zugarmierung in den zwei benachbarten Rissequerschnitten zur Folge. Dies bewirkt eine gegenüber dem Fall reiner Biegebeanspruchung andere Verteilung der Verbundspannungen. Die Stelle, an welcher die Verbundspannungen das Vorzeichen wechseln, respektive wo die Verschiebung zwischen Beton und Zugarmierung verschwindet, fällt nicht mehr in die Mitte zwische 
den Rissen, sondern sie verschiebt sich gegen den Riss mit der kleineren Zugkraft hin. Aus Gleichgewichtsgrïnden gilt:

$\Delta z_{i, i+1}=\left[\int_{0}^{x_{\max i, i+1}} \tau_{v}(x) \cdot d x+\int_{0}^{x_{\max i+1, i}} \tau_{v}(x) \cdot d x\right] \cdot \Sigma u$

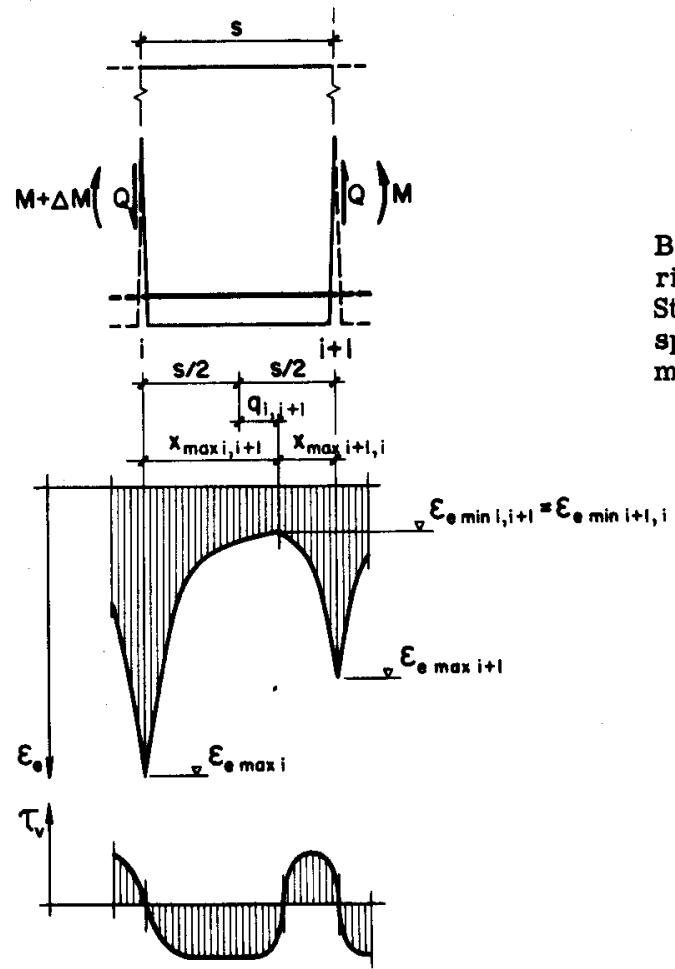

Bild 17

Bereich zwischen zwei Biegerissen; genereller Verlauf der Stahldehnungen und Verbundspannungen bei variablem Biegemoment

In Gleichung ( 73 ) bedeuten die Grössen $x_{\max }$ die Abstănde des Verschiebungs Nullpunktes bis zu den Rissen. Da diese Strecken beidseits eines Risses verschieden sind, werden sie durch einen zweiten Index eindeutig bezeichnet. 
Wird die Lage des Verschiebungs-Nullpunktes gegenüber dem Fall reiner Biegung durch die Strecke $q_{i, i+1}$ charakterisiert, so gilt ferner:

$$
\begin{aligned}
& x_{\max i, i+1}=\frac{s}{2}+q_{i, i+1} \\
& x_{\max i+1, i}=\frac{s}{2}-q_{i, i+1}
\end{aligned}
$$

Im allgemeinen können die Strecken $q$ beziehungsweise $x_{\max }$ nicht explizite berechnet werden. Wie noch gezeigt wird, ist eine Iteration nötig.

Verschiedene der für den Fall reiner Blegung aufgestellten Grössen und Formeln müssen noch allgemeiner definiert werden. Die wichtigsten Aenderungen sind, dass anstelle von ( 23 ) gilt:

$$
\begin{aligned}
& w_{i}=v_{\max i, i-1}+v_{\max i, i+1} \\
& v_{\max i, i+1}=\int_{0}^{x_{\max } i, i+1} \varepsilon_{e^{(x) \cdot d x}} \\
& v_{\max i, i-1}=\int_{0}^{x} \max i, i-1 \quad \varepsilon_{e}(x) \cdot d x
\end{aligned}
$$

Das Vorgehen zur verfeinerten Berechnung der einzelnen Rissweiten in einem plastischen Gelenk wird ähnlich wie im vorangehenden Abschnitt dargestellt. Es sind im kritischen Riss (Riss Nr. 1) wiederum sowohl $w_{k r}$ als auch $w_{k r b} z u$ berechnen, wobei der kleinere Wert massgebend ist.

\section{Versagen_der_Zugarmierung}

Erster, kritischer Riss:

1. $\sigma_{e \max 1}=\beta_{z}$

2. $x_{\max 1,2}$ beziehungsweise $q_{1,2}$ schätzen 
3. Aus Bild 8 (oder ähnlichen Darstellungen beziehungsweise Tabellen) folgen: $\sigma_{e \min 1,2}, v_{\max 1,2}$

Zweiter Riss:

4. $\mathrm{x}_{\max 2,1}=\mathrm{s}-\mathrm{x}_{\max 1,2}$

5. Aus Bild 8 oder dergleichen folgen mit $\sigma_{\text {e min } 2,1}=\sigma_{\text {e min } 1,2}$ : $\sigma_{e \max 2}, v_{\max 2,1}$

6. Kontrolle nach ( 71$)$ :

$$
\sigma_{e \max 1}-\sigma_{e \max 2}=\frac{\Delta Z_{1,2}}{F_{e}}
$$

7. Wiederholung der Rechnung mit verbesserten Werten $x_{\max 1,2}$ beziehungsweise $q_{1,2}$ bis $\mathrm{zu}$ genügender Uebereinstimmung

8. $x_{\max 2,3}$ beziehungsweise $q_{2,3}$ schätzen, und so weiter analog wie im ersten Riss, in allen Rissen des Gelenkes.

\section{Versagen des Betons}

Erster, kritischer Riss:

1. Aus ( 52 ) beziehungsweise ( 53 ) folgt $w_{k r b}$

2. $v_{\max 1,2} \cong \frac{\mathrm{w}_{\mathrm{krb}}}{2} \operatorname{nach}(23)$

3. $x_{\max 1,2}$ beziehungsweise $q_{1,2}$ schätzen

4. Aus Bild 8 oder dergleichen folgen: $G_{e \max 1}, G_{e \min 1,2}$

\section{Zweiter Riss:}

Weitere Berechnung wie bei Versagen der Zugarmierung. 
Die Berechnung von $w_{k r b}$ nach ( 52 ) beziehungsweise ( 53 ) gilt für die Annahme, dass die Betonstauchungen am Rand der Druckzone symmetrisch zum Querschnitt mit dem kritischen Riss verlaufen. Bei einer innerhalb des plastischen Gelenkes variablen Momentenbeanspruchung ist dies streng genommen nicht der Fall. Ferner tritt mit der Verwendung von ( 23 ) eine weitere "Ungenauigkeit" auf, da die Verschiebungen beidseits des kritischen Risses im allgemeinen nicht gleich gross sind. Dles wăre nur der Fall, wenn die Momente in den beiden dem kritischen Riss benachbarten Rissen ihrerseits gleich gross wären. Beide Ungenauigkeiten sind jedoch angesichts der für die Berechnung des kritischen Winkels infolge Versagen des Betons getroffenen Annahmen und der in Wirklichkeit vorhandenen Streuungen unbedeutend.

Einfacher als im Falle eines allgemeinen Verbundgesetzes und beliebigen Spannungs-Dehnungs-Diagrammes wird die Berechnung der Verformungen in der Zugzone für Riegeltorstahl, beziehungsweise wenn

$$
\underset{\mathbf{v}}{\tau}(\mathbf{v}) \cong \text { konst. }=\tau_{\mathbf{v}}^{*}
$$

gesetzt werden kann. Die Strecke q ergibt sich explizite aus ( 73 ):

$$
q_{i, i+1}=\frac{\Delta z_{i, i+1}}{2 \cdot \tau_{v}^{*} \cdot \pi \cdot \Sigma d}
$$

Damit kann nach ( $74 a$ ) beziehungsweise $(74 b)$ jeweils die Strecke $x_{\max }$ direkt berechnet werden, und die vorher nötige Iteration entfällt.

Analog wie früher müssen auch hier die Formeln des Abschnittes 2.21 teilweise allgemeiner angeschrieben werden. Zum Beispiel wird aus ( 26 ):

$$
\Delta \sigma_{e \text { tot } i, i+1}=\tau_{v}^{*} \cdot \frac{4}{d} \cdot x_{\max i, i+1}
$$

Die Berechnung der Rissweiten bei Versagen des Betons wie der Zugarmierung erfolgt analog wie vorgehend im Falle allgemeiner Grundlagen. Nebst dem Wegfall der Iteration für $x_{\max }$ ist jedoch anstelle von Bild 8 die Tafel 1 zu verwenden. 


\subsection{Formeln für spezielle Grundlagen}

Aus den bisherigen Darlegungen geht hervor, dass die Zusammenhänge in einem Biegeriss-Gelenk äusserst kompliziert sind und die kritischen Gelenkwinkel von den verschiedensten Parametern beeinflusst werden. Für diese Winkel lassen sich deshalb im Falle allgemeiner Verhältnisse keine expliziten Formeln aufstellen. Dies ist jedoch möglich für etwas spe zielle und idealisierte Grundlagen und wird im folgenden durchgeführt. Die resultierenden Formeln sollen nicht für einen praktischen Gebrauch dienen, da meist nicht alle Voraussetzungen erfüllt sind. Vielmehr lässt sich an ihnen der Einfluss einer Variation einzelner Parameter bedeutend besser überblicken als bei allgemeinen Verhältnissen.

Vorerst wird eine Formel für die totale Rissweite in einem plastischen Gelenk entwickelt. Anschliessend werden die entsprechenden Ausdrücke für den kritischen Gelenkwinkel sowohl für Versagen der Zugarmierung wie auch des Betons hergeleitet. Dabei müssen gewisse Vereinfachungen und Näherungen vorausgesetzt werden, welche ausschliesslich in bestimmten Parameterbereichen zulässig sind. Trotzdem ergibt sich eine erheblich verbesserte Uebersicht uber die grundsätzlichen Abhängigkeiten.

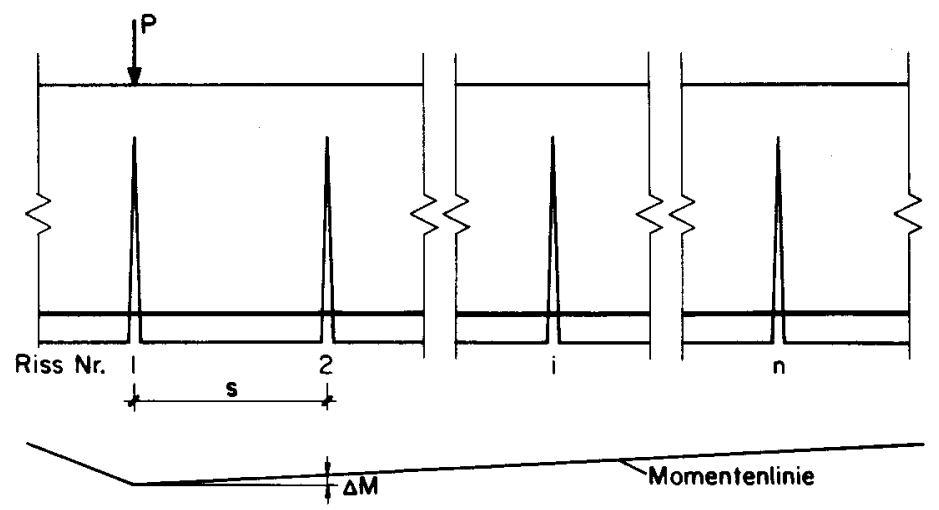

Bild 18

Biegeriss-Gelenk bei Einzellast 
In Bild 18 ist ein Biegeriss-Gelenk im Bereich einer Einzellast dargestellt. Definitionsgemäss sind diejenigen $\mathrm{n}$ Risse eingezeichnet und numeriert worden, in denen plastische Verformungen der Zugarmierung erfolgen, das heisst $\varepsilon_{e} \geqslant \varepsilon_{f}$ ist. Für die Zugarmierung wird das idealisierte Spannungs-Dehnungs-Diagramm nach Bild 19 angenommen.

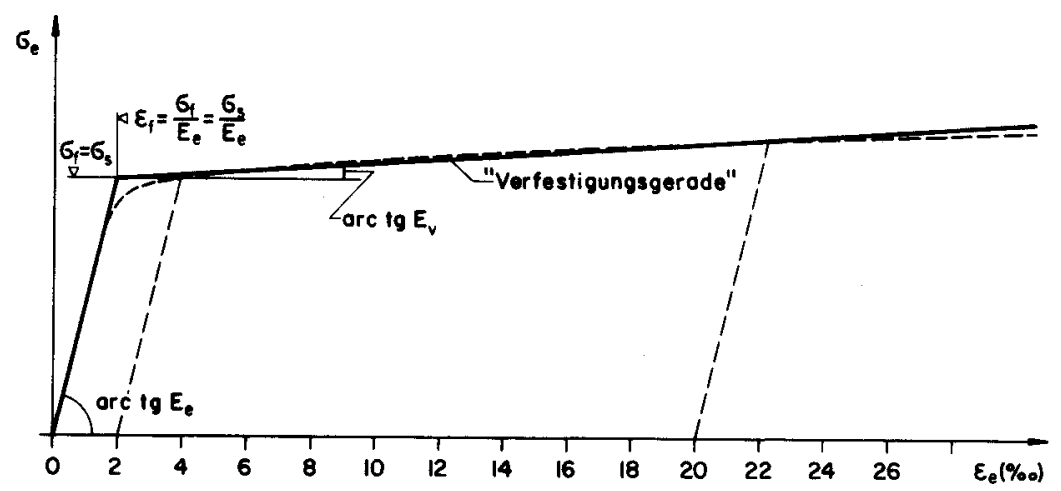

Bild 19

Idealisiertes Spannungs-Dehnungs-Diagramm und Anfangsbereich des normierten Diagrammes für Riegeltorstahl gemäss Bild 10

Dieses besteht aus zwei Geraden. Die Neigungen derselben entsprechen dem Elastizitätsmodul $E_{e}$ respektive dem Verfestigungsmodul $E_{v}$. Für $E_{e}$ gilt der normale Wert $\mathrm{E}_{\mathrm{e}}=2^{\prime} 100^{\prime} 000 \mathrm{~kg} / \mathrm{cm}^{2}$. Für Riegeltorstahl ergibt $\operatorname{sich} \mathrm{E}_{\mathrm{v}} \cong 32^{\prime} 000$ $\mathrm{kg} / \mathrm{cm}^{2}$, wenn im normierten Diagramm gemäss Bild 10 die Fixpunkte $\varepsilon_{\mathrm{f}}, \sigma_{\mathrm{f}}$ (Bild 2) sowie $\varepsilon_{\text {plast }}=20 \% 0 \mathrm{mit}$ dem zugehörigen Wert für $G_{\mathrm{e}}$ zugrundegelegt werden. Das idealisierte Spannungs-Dehnungs-Diagramm entspricht mit $\mathrm{E}_{\mathrm{v}}=$ $32,000 \mathrm{~kg} / \mathrm{cm}^{2}$ somit demjenigen des Riegeltorstahles für ungefähr

$$
\varepsilon_{\text {plast }} \leq 30^{\circ} / 00
$$

Nach Gleichung ( 13 ) gilt für die Rissweite des Risses i: 


$$
w_{i}=\varepsilon_{e \max i} \cdot \kappa_{i} \cdot \mathbf{s}
$$

Die Summe der Rissweiten im ganzen Gelenk beträgt:

$$
\sum_{1}^{n} w_{i}=s \cdot \sum_{1}^{n} \varepsilon_{e \max i} \cdot \kappa_{i}
$$

Der Unterschied zwischen der maximalen Stahldehnung in zwei benachbarten Rissen kann dank der Einführung der "Verfestigungsgeraden" wie folgt explizit formuliert werden:

$$
\Delta \varepsilon_{\mathrm{e} \max }=\frac{\Delta \mathrm{Z}}{\mathrm{F}_{\mathrm{e}}} \cdot \frac{1}{\mathrm{E}_{\mathrm{v}}}
$$

Daraus wird mit ( 72 ):

$$
\Delta \varepsilon_{\text {emax }}=Q \cdot \frac{s}{y} \cdot \frac{1}{E_{v} \cdot F_{e}}
$$

Für das Folgende ist es zweckmässig, wenn ( 79 ) noch mit der nominellen Fliesspannung $\sigma_{\mathrm{f}}$ erweitert wird. Mit der nominellen Fliesskraft in der Zugarmierung,

$$
z_{f}=\sigma_{f} \cdot F_{e}
$$

wird

$$
\Delta \varepsilon_{\mathrm{e} \max }=\frac{\sigma_{f}}{E_{v}} \cdot \frac{Q}{Z_{f}} \cdot \frac{s}{y} .
$$

Damit ergibt sich die Anzahl der Risse mit plastischen Verformungen der Zugarmierung nach Bild $20 \mathrm{zu}$ 


$$
n=1+\text { entier }\left[\frac{\varepsilon_{e \max 1}-\varepsilon_{f}}{\Delta \varepsilon_{e \max }}\right] \text {. }
$$

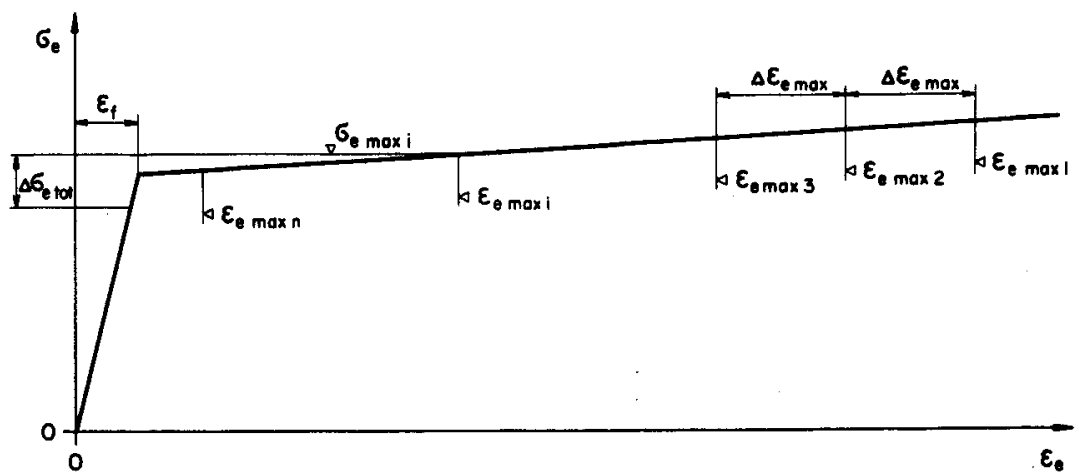

\section{Bild 20}

Bezeichnungen und Zusammenhänge im idealisierten Spannungs-Dehnungs-Diagramm

Ferner gilt für die maximalen Stahldehnungen in den einzelnen Rissen:

$$
\begin{array}{cccc}
\varepsilon_{e \max 2} & =\varepsilon_{e \max 1} & -\Delta \varepsilon_{e \max } \\
\varepsilon_{e \max 3} & = & \varepsilon_{e \max 1} & -2 \cdot \Delta \varepsilon_{e \max } \\
\vdots & \vdots & \vdots \\
\varepsilon_{e \max 1} & = & \varepsilon_{e \max 1} & -(i-1) \cdot \Delta \varepsilon_{e \max } \\
\vdots & \vdots & \vdots \\
\varepsilon_{e \max n} & =\varepsilon_{e \max 1}-(n-1) \cdot \Delta \varepsilon_{e \max }
\end{array}
$$

Werden diese Grössen in ( 77 ) eingesetzt, so erhält man: 


$$
\sum_{1}^{n} w_{i}=s \cdot\left[\varepsilon_{e \max 1} \cdot \sum_{1}^{n} \kappa_{i}-\Delta \varepsilon_{e \max } \cdot \sum_{1}^{n}(i-1) \cdot \kappa_{i}\right]
$$

Der Verbundkoeffizient $\kappa_{i}$, als Verhältnis der mittleren zur maximalen Stahldehnung aufgefasst, beträgt:

$$
\kappa_{i}=\frac{\int_{e \min i}^{\sigma_{e \max i}} \varepsilon_{e}\left(\sigma_{e}\right) \cdot d \sigma_{e}}{\varepsilon_{e \max i} \cdot \Delta \sigma_{e \text { tot } i}}
$$

Darin ist für eine konstante Verbundspannung nach ( 24 ) gemäss ( 26 ):

$$
\Delta \sigma_{\text {e tot } \mathrm{i}}=\tau_{\mathrm{v}}^{*} \cdot \frac{4}{\mathrm{~d}} \cdot \frac{\mathrm{s}}{\mathbf{2}}=\text { konst. }=\Delta \sigma_{\mathrm{etot}}
$$

Unter der Voraussetzung, dass

$$
\left(\varepsilon_{\text {emax } 1}-\varepsilon_{f}\right) \cdot E_{v} \leq \Delta G_{\text {etot }},
$$

wofür auch

$$
\frac{\mathbf{s}}{\mathbf{d}} \geq \frac{\varepsilon_{\mathrm{emax} 1}-\varepsilon_{f}}{2 T_{v}^{*}} \cdot \mathbf{E}_{v}
$$

stehen kann, wird nach Bild 20:

$\kappa_{i}=\frac{\left(\varepsilon_{e \max i}-\varepsilon_{f}\right)^{2} \cdot \frac{E_{v}}{2}+\varepsilon_{f} \cdot \Delta \sigma_{e t o t}-\left[\Delta \sigma_{e t o t}-\left(\varepsilon_{e \max i}-\varepsilon_{f}\right) E_{v}\right]^{2} \cdot \frac{1}{2 E_{e}}}{\varepsilon_{e \max i} \cdot \Delta \sigma_{e \text { tot }}}$

Hierfür kann als Näherung gesetzt werden: 


$$
\kappa_{i} \cong \frac{\varepsilon_{\text {emaxi }} \cdot \frac{E_{v}}{2}}{\Delta \sigma_{\text {etot }}}
$$

Die Differenz $z$ wischen ( 87 ) und ( 88 ) wächst mit kleiner werdendem $\varepsilon_{\text {e } \max i}$. Der entstehende Fehler ist jedoch für das Schlussresultat unerheblich.

( 81$)$ in ( 88$)$ ergibt:

$$
\kappa_{i} \cong \frac{E_{v}}{2 \cdot \Delta \sigma_{e t o t}} \cdot\left[\varepsilon_{e \max 1}-(i-1) \cdot \Delta \varepsilon_{e \max }\right]
$$

Durch Einsetzen von ( 89 ) in ( 82 ) wird die Summe der Rissweiten:

$$
\begin{aligned}
\sum_{1}^{n} w_{i}= & \frac{s \cdot E_{V}}{2 \cdot \Delta \sigma_{e t o t}} \cdot\left\{\varepsilon_{e \max 1} \cdot\left[\varepsilon_{e \max 1} n-\Delta \varepsilon_{e \max } \cdot \sum_{1}^{n}(i-1)\right]-\right. \\
& \left.-\Delta \varepsilon_{e \max } \cdot\left[\varepsilon_{e \max 1} \cdot \sum_{1}^{n}(i-1)-\Delta \varepsilon_{e \max } \cdot \sum_{2}^{n}(i-1)(i-1)\right]\right\}
\end{aligned}
$$

Nach geeigneten Umformungen sowie Einsetzen von Gleichung ( 84 ) erhält man schliesslich den entsprechenden Gelenkwinkel nach ( 9 ):

$\Theta=\frac{E_{v}}{4 \tau_{v}^{*}} \cdot\left[\frac{d}{h-z}\right] \cdot\left[\varepsilon_{e \max 1}^{2} \cdot n-\varepsilon_{e \max 1} \cdot \Delta \varepsilon_{e \max }(n-1) \cdot n+\Delta \varepsilon_{e \max }^{2} \cdot \frac{(n-1) \cdot n(2 n-1)}{6}\right]$

Der gesuchte kritische Gelenkwinkel $\Theta_{\mathrm{kr}}$ ist erreicht, wenn entweder Versagen des Stahles oder des Betons eintritt. Bei Versagen des Stahles gilt nach ( 36 ) $\varepsilon_{\text {emax } 1}=\varepsilon_{\text {er }}$. Damit wird aus $(90)$ und ( 80$)$ der kritische Gelenkwinkel für Versagen des Stahles: 
$\theta_{\mathrm{kr} e}=\frac{E_{v}}{4 \tau_{\mathrm{v}}^{*}} \cdot\left[\frac{\mathrm{d}}{\mathrm{h}-\mathrm{z}}\right] \cdot\left[\varepsilon_{\mathrm{er}}^{2} \cdot n-\varepsilon_{\mathrm{er}} \cdot \Delta \varepsilon_{\mathrm{emax}} \cdot(\mathrm{n}-1) \cdot \mathrm{n}+\Delta \varepsilon_{\mathrm{emax}}^{2} \cdot \frac{(\mathrm{n}-1) \cdot \mathrm{n} \cdot(2 \mathrm{n}-1)}{6}\right]$

mit

$$
n=1+\text { entier }\left[\frac{\varepsilon_{\text {er }}-\varepsilon_{f}}{\Delta \varepsilon_{e \max }}\right]
$$

und

$$
\Delta \varepsilon_{\text {emax }}=\frac{\sigma_{f}}{E_{v}} \cdot \frac{Q}{Z_{f}} \cdot \frac{s}{y}
$$

Diese Formeln dürfen allerdings nicht für Versagen des Stahles bei Riegeltorstahl verwendet werden. Denn das idealisierte Spannungs-Dehnungs-Diagramm nach Bild 19 gilt gemäss ( 76 ) nur bis ungefähr $\varepsilon_{e}=30 \% 0$, während $\lambda_{G l}$ von Torstahl etwa 50 bis $80 \%$ o beträgt.

Im Falle des Versagens des Betons wird - stets gleiche Betondruckstauchung $\varepsilon_{\mathrm{br}}$ vorausgesetzt - die im breitesten Riss erreichbare maximale Stahldehnung $\varepsilon_{\text {emax } 1}$ von verschiedenen Grössen beeinflusst. Aufgrund der Formeln (13a), ( 84 ) und ( 88 ) ergibt sich im Riss Nr. 1:

$$
\varepsilon_{\max 1}=\sqrt{w_{1} \cdot \frac{4 \cdot \tau_{v}^{*}}{E_{v} \cdot d}}
$$

Setzt man darin für $w_{1}$ die kritische Rissweite $w_{k r b}$ nach ( 52 ) und ( 53 ) ein, so erhält man, nach Umformung der Bedingungen betreffend das Verhältnis $\mathrm{z} / \mathrm{s}$, die folgenden Ausdrücke:

$\frac{z}{y} \leq \frac{s}{y}: \quad \varepsilon_{\max 1}=\sqrt{\varepsilon_{b r} \cdot\left[\frac{y}{z}\right]^{1 / 3} \cdot\left[\frac{s}{y}\right]^{1 / 3} \cdot\left[\frac{h-z}{d}\right] \cdot \frac{4 \tau_{v}^{*}}{E_{v}}}$ 
$\frac{z}{y} \geq \frac{s}{y}: \quad \varepsilon_{e \max 1}=\sqrt{\varepsilon_{b r} \cdot\left[\frac{y}{z}\right] \cdot\left[\frac{s}{y}\right] \cdot\left[\frac{h-z}{d}\right] \cdot \frac{4 \tau_{v}^{*}}{E_{v}}}$

Somit ergibt sich der kritische Gelenkwinkel für Versagen des Betons wie folgt:

$$
\begin{aligned}
\Theta_{\mathrm{krb}}=\frac{E_{\mathrm{v}}}{4 \tau_{\mathrm{v}}^{*}} \cdot\left[\frac{\mathrm{d}}{h-\mathrm{z}}\right] \cdot & {\left[\varepsilon_{\mathrm{e} \max 1}^{2} \cdot \mathrm{n}-\varepsilon_{\mathrm{e} \max 1} \cdot \Delta \varepsilon_{\mathrm{e} \max } \cdot(\mathrm{n}-1) \cdot \mathrm{n}\right.} \\
& \left.+\Delta \varepsilon_{\mathrm{emax}}^{2} \cdot \frac{(\mathrm{n}-1) \cdot \mathrm{n} \cdot(2 \mathrm{n}-1)}{6}\right]
\end{aligned}
$$

mit

$$
n=1+\text { entier }\left[\frac{\varepsilon_{\text {emax } 1}-\varepsilon_{f}}{\Delta \varepsilon_{e \max }}\right]
$$

und

$$
\Delta \varepsilon_{e \max }=\frac{\sigma_{f}}{E_{v}} \cdot \frac{Q}{Z_{f}} \cdot \frac{s}{y}
$$

sowie mit ( 92 ) respektive ( 93 ).

Es muss hier nochmals hervorgehoben werden, dass die obigen Formeln für den kritischen Gelenkwinkel infolge Versagen des Stahles respektive des Betons auf den nachstehenden Voraussetzungen basieren:

- Idealisiertes Spannungs-Dehnungs-Diagramm nach Bild 19 (Naherung für Riegeltorstahl bis etwa $\left.\varepsilon_{\mathrm{e}}=30 \% \mathrm{o}\right)$,

- Konstante Querkraft längs des Gelenkes,

- Konstante Verbundspannung, das heisst die Verbundspannung ist unabhängig von der Relativverschiebung der Längsarmierung gegenüber dem Beton,

- Năherung für den Verbundkoeffizienten $\kappa$ nach ( 88 ) mit Bedingung ( 85 ) respektive ( 86 ).

Obwohl diese Voraussetzungen meist erhebliche Einschränkungen darstellen, werden 
es die hergeleiteten Beziehungen ermöglichen, im nächsten Abschnitt den Einfluss einiger Parameter auf die kritischen Gelenkwinkel darzulegen.

\subsection{Diskussion der Ergebnisse}

In den bisherigen Ausführungen über das Biegeriss-Gelenk wurde dargelegt, wie die kritischen Gelenkwinkel infolge Versagen des Stahles und des Betons ermittelt werden können. In der folgenden Diskussion der Ergebnisse wird vorerst der Einfluss wichtiger Parameter auf diese Winkel untersucht. Anschliessend wird die Theorie anhand von Versuchsresultaten überprüft. Schliesslich wird ein Vergleich mit der ublichen Betrachtungsweise, welche die Hypothese vom Ebenbleiben der Querschnitte zur Grundlage hat, vorgenommen.

\subsection{Einfluss wichtiger Parameter auf die kritischen Gelenkwinkel}

Auf die kritischen Gelenkwinkel haben verschiedene Parameter einen Einfluss. Es sind dies neben den geometrischen Grössen des Querschnittes $h, h^{\prime}, b, b_{o}, F_{e}$, $F_{e}, A, F_{q}$ und der Betonfestigkeit $\beta$ vor allem die folgenden:

- Veränderung des Momentes längs des Gelenkes (Querkraft, Momentengradient),

- Rissabstand,

- Form des Spannungs-Dehnungs-Diagrammes des Stahles (insbesondere Dehnungsund Verfestigungseigenschaften),

- Verbundeigenschaften (Verbundgesetz),

- Stabdurchmesser.

Zur Untersuchung des Einflusses dieser Parameter werden die Beziehungen des Abschnittes 2.42 herangezogen. Obwohl diese nur aufgrund spezieller Annahmen und Voraussetzungen hergeleitet werden konnten, kommen dort die einzelnen $\mathrm{Pa}$ rameter meist implizite in komplizierteren Ausdrücken vor. Um eine Aussage 
beziehungsweise Darstellung erarbeiten zu können, wird es daher nötig sein, jeweils für die nicht variierten Grössen plausible Zahlenwerte einzusetzen. Die quantitativen Ergebnisse sind deshalb stets im Sinne von Beispielen zu verstehen und dürfen nicht verallgemeinert werden. Hingegen lässt sich die Tendenz, wie die kritischen Gelenkwinkel durch die einzelnen Parameter beeinflusst werden, meist recht gut feststellen. Es wird daher möglich sein, trotz der verschiedenen Einschränkungen einige allgemeinere Schlüsse zu ziehen, gewisse Beobachtungen zu erklären und vorher mehr nur erahnte Erkenntnisse besser zu belegen.

\section{a) Einfluss von Querkraft und Rissabstand auf $\Theta_{\mathrm{kr} \mathrm{e}}$}

Zur Untersuchung des Einflusses von Querkraft und Rissabstand auf den kritischen Gelenkwinkel infolge Versagen der Zugarmierung werden die Ausdrücke (90a) mit ( 80a) und (79a) verwendet. In der Gleichung ( 79a) kommen die Quotienten $Q / Z_{f}$ und $s / y$ als unabhängige Variable vor. Das Verhältnis Querkraft $Q$ zu Fliesskraft $Z_{f}$ in der Zugarmierung charakterisiert die Bedeutung der Querkraft im Biegeriss-Gelenk ähnlich wie das Momenten-Schub-Verhältnis im Schubriss-Gelenk (siehe 3.). Die Beziehung Rissabstand s zu innerem Hebelarm $y$ ist wesentlich für die Geometrie des Gelenkes. Beide Quotienten haben einen ähnlichen Einfluss auf $\mathbf{Q}_{\mathbf{k r}}$.

In Bild 21 sind die Zusammenhänge graphisch dargestellt. Dazu wurden die folgenden Zahlenwerte angenommen:

$$
\begin{array}{llrl}
\sigma_{f}=4000 \mathrm{~kg} / \mathrm{cm}^{2}, & E_{v}=32.000 \mathrm{~kg} / \mathrm{cm}^{2}, & \tau_{v}^{*}=70 \mathrm{~kg} / \mathrm{cm}^{2}, \\
\varepsilon_{\mathrm{er}}=30 \% \mathrm{o}, & \varepsilon_{\mathrm{f}}=1,91 \% 0, & \mathrm{~d} /(\mathrm{h}-\mathrm{z})=0,06 .
\end{array}
$$

Dafür lautet die Bedingung ( 86 ):

$$
\frac{s}{d} \geq 6,4
$$




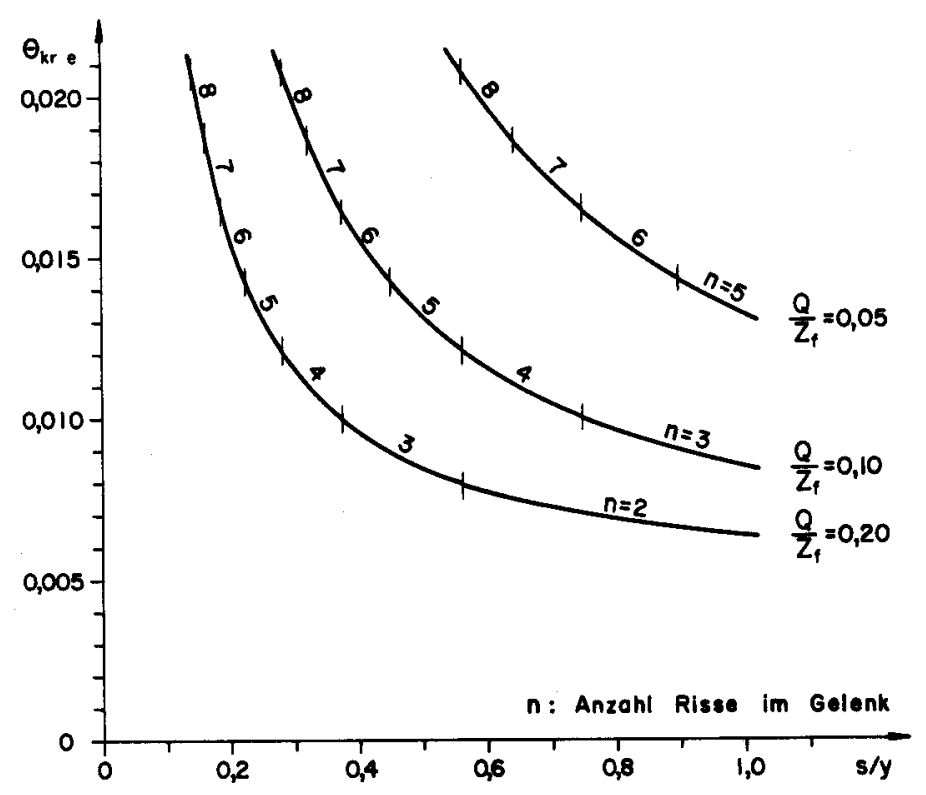

\section{Bild 21}

Einfluss von Querkraft und Rissabstand auf $\Theta_{\mathrm{kr} \mathrm{e}}$ (Beispiel)

In Bild 21 ist der Verlauf von $\theta_{\mathrm{kr}} \mathrm{e}$ für drei verschiedene Werte $Q / Z_{\mathrm{f}}$ im $B e-$ reich $s / y=0,2 \div 1,0$ aufgetragen. In den Kurven besteht stets dort eine gewisse, im Bild allerdings nur schwach erkennbare Unstetigkeit, wo sich die Anzahl der Risse des Gelenkes verändert. Diese Anzahl wird mit kleinerem Rissabstand grösser.

Man erkennt, wie sehr $\Theta_{\mathrm{kr} \text { e }}$ - bei gleichbleibender Querkraft - vom Rissabstand abhängt. Denn je kleiner der Rissabstand ist, desto grösser wird der kritische Gelenkwinkel. Die Zunahme ist im Bereich $s / y=0,2 \div 0,5$ besonders ausge-

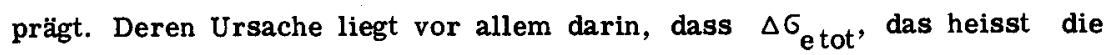
Differenz zwischen der maximalen Spannung im Riss und der minimalen Spannung zwischen den Rissen umso geringer ausfällt, je kleiner der Rissabstand ist. Da- 
durch nimmt der Verbundkoeffizient und damit die mittlere Dehnung stark zu. Aber auch die Querkraft ist von grossem Einfluss auf $\Theta_{\mathrm{kr}}$. Sie bestimmt zusammen mit der Form des Spannungs-Dehnungs-Diagramms massgeblich die Unterschiede zwischen den maximalen Zugkräften und Dehmungen in benachbarten Rissen. Damit ist sie auch weitgehend verantwortlich für die Ausdehnung des plastischen Gelenkes. Gleichbleibender Rissabstand vorausgesetzt, entstehen mit abnehmender Querkraft mehr Risse mit plastischen Stahldeformationen. Ausserdem wird die Breite der nebst dem kritischen Riss vorhandenen Risse stark beeinflusst. Bei verhältnismässig grosser Querkraft können sich die plastischen Deformationen auf nur 1 bis 2 Risse beschränken. Der Winkel $\theta_{k r e}$ făllt entsprechend gering aus. indem er gleich wie oder nur wenig grösser als der kritische Risswinkel wird. Bei kleinerer Querkraft hingegen sind in mehr Rissen grössere Risswinkel vorhanden, deren Summe einen vergrösserten Wert für $\Theta_{\text {kre }}$ liefert.

\section{b) Einfluss von Querkraft und Rissabstand auf $\theta_{\mathbf{k r ~ b}}$}

Der Einfluss von Querkraft und Rissabstand auf den kritischen Gelenkwinkel infolge Versagen des Betons wird ähnlich wie im vorangehenden Abschnitt, jedoch anhand der Gleichungen ( $90 \mathrm{~b}$ ), ( 80 ), ( 79a ) und ( 92 ) respektive ( 93 ) behandelt. Der graphischen Darstellung Bild 22 liegen die folgenden Zahlenwerte zugrunde:

$$
\begin{array}{ll}
\sigma_{f}=4000 \mathrm{~kg} / \mathrm{cm}^{2}, & E_{v}=32,000 \mathrm{~kg} / \mathrm{cm}^{2}, \quad \tau_{v}^{*}=70 \mathrm{~kg} / \mathrm{cm}^{2}, \\
\varepsilon_{f}=1,91 \% 0, & \varepsilon_{b r}=3,50 \% 0, \quad z / y=0,20, \quad \mathrm{~d} /(\mathrm{h}-\mathrm{z})=0,06 .
\end{array}
$$

Für $s / y=0,2$ gilt somit die Gleichung ( 92 ). Für $s / y=1,0$ wird $\varepsilon_{\text {e } \max 1}=$ $29,55 \%$ und liegt somit gerade noch innerhalb der Gültigkeitsgrenze $(76)$ des idealisierten Spannungs-Dehnungs-Diagrammes für Riegeltorstahl.

Bild 22 weist gewisse Aehnlichkeiten mit Bild 21 auf. Man erkennt jedoch, dass der Rissabstand nicht mehr denselben grossen Einfluss auf $\Theta_{\mathrm{krb}}$ hat, wie dies auf $\theta_{\text {kre }}$ der Fall war. Mit kleinerem Rissabstand verringert sich die beim 


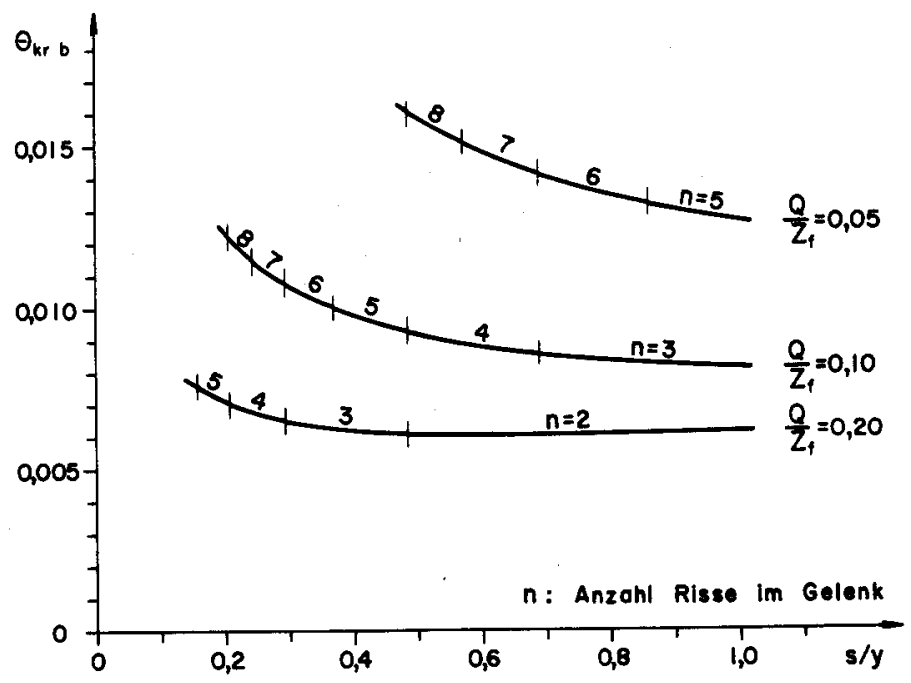

Bild 22

Einfluss von Querkraft und Rissabstand auf $\Theta_{k r b}$ (Beispiel)

Bruch des Betons im kritischen Riss vorhandene Stahldehnnng $\varepsilon_{e \max 1}$ nach ( 92 ), womit der dortige Risswinkel nicht mehr wie beim Versagen des Stahles unabhängig vom Rissabstand ist. Für Verhältnisse $Q / Z_{f}=0,1 \div 0,2$ wird dadurch die Wirkung des vergrösserten Verbundkoeffizienten weitgehend kompensiert. Erst für verhältnismässig kleine Querkräfte ergibt sich noch eine merkbare Steigerung von $\theta_{k r b}$, die jedoch geringer ausfällt als beim Versagen des Stahles.

Die Wirkung der Querkraft auf $\Theta_{k r b}$ ist ähnlich wie auf $\Theta_{k r e}$. 


\section{c) Einfluss der Gleichmassdehnung auf $\Theta_{\mathrm{kr}}$}

Von wesentlicher Bedeutung für den kritischen Gelenkwinkel infolge Versagen des Stahles ist die Dehnung der Zugarmierung beim Beginn der Einschnürung. Diese ist nach ( 40 ) etwa gleich der Gleichmassdehnung. Die Zusammenhänge können mit Hilfe der Formeln (90a), ( $80 \mathrm{a}$ ) und (79a) abgeschätzt werden. Dazu werden die folgenden Zahlenwerte benützt:

$$
\begin{aligned}
& \sigma_{\mathrm{f}}=4000 \mathrm{~kg} / \mathrm{cm}^{2}, \quad E_{v}=32,000 \mathrm{~kg} / \mathrm{cm}^{2}, \quad \tau_{v}^{*}=70 \mathrm{~kg} / \mathrm{cm}^{2}, \\
& \varepsilon_{f}=1,91 \% 0, \quad Q / Z_{f}=0,20, \quad s / y=0,50, \quad \mathrm{~d} /(\mathrm{h}-\mathrm{z})=0,06 .
\end{aligned}
$$

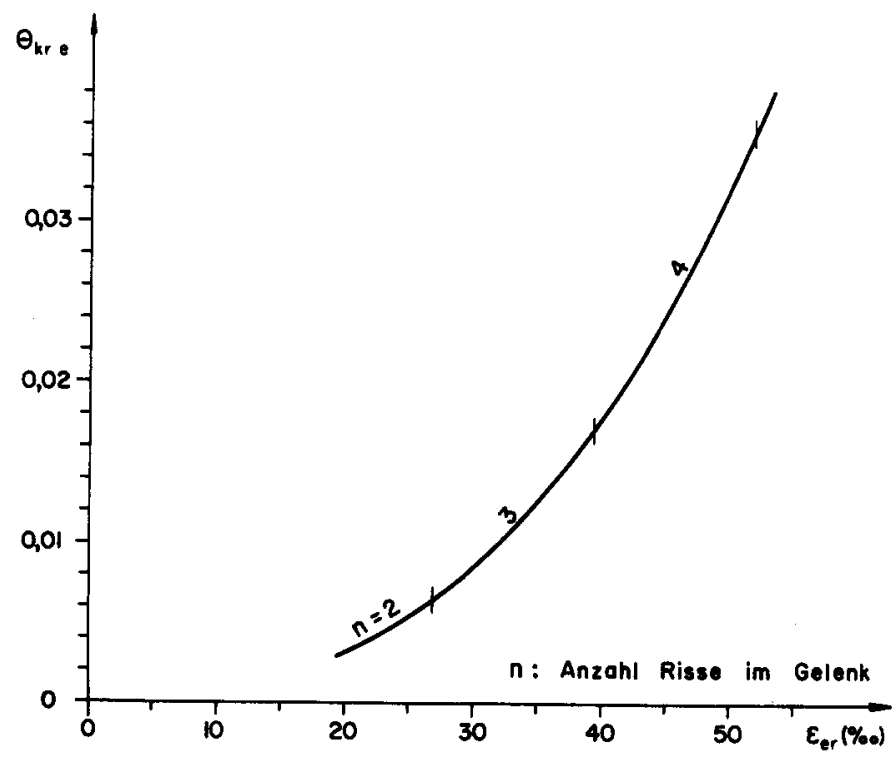

$\underline{\text { Bild } 23}$

Einfluss der Gleichmassdehnung auf $\Theta_{\mathrm{kr} e}$ (Beispiel)

Das Ergebnis ist in Bild 23 dargestellt. Die kritische Dehnung $\varepsilon_{\mathrm{er}} \cong \lambda_{\mathrm{Gl}}$ wur- 
de variiert von 20 bis $50 \%$. Für $\varepsilon_{\text {er }}>30 \%$ o liegt somit ein vollkommen fiktives Spannungs-Dehnungs-Diagramm entsprechend Bild 19, jedoch mit Fortsetzung der Verfestigungsgeraden, zugrunde. Der überproportionale Anstieg der Kurve in Bild 23 steht mit diesen Annahmen in engem Zusammenhang. Denn mit zunehmendem $\varepsilon_{\text {e } r}$ vergrössert sich hier der Verbundkoeffizient $\kappa$ enorm. Für $\varepsilon_{\text {er }}=50 \%$ o wir denn auch ( 86 ):

$$
\frac{s}{d} \geq 11,0
$$

Diese Bedingung ist praktisch kaum einzuhalten, sodass die Darstellung Bild 23 bloss in der Tendenz zutrifft. Bei gekrümmtem Verlauf des Verfestigungsbereiches im Spannungs-Dehnungs-Diagramm, wie zum Beispiel bei Riegeltorstahl, nimmt $\kappa$ mit zunehmendem $\varepsilon_{\mathrm{er}}$ eher ab, womit der Anstieg von $\theta_{\mathrm{kr}}$ geringer ist als in Bild 23. Ueberhaupt ist die Form des gesamten Spannungs-DehnungsDiagrammes von grösster Bedeutung für den dargestellten Zusammenhang (siehe auch nächster Abschnitt). Immerhin gilt erwartungsgemäss, dass mit grösserer Gleichmassdehnung der kritische Gelenkwinkel infolge Versagen der Zugarmierung anwächst. Entsprechend verringert sich die Gefahr eines Versagens des Stahles vor demjenigen des Betons (vergleiche 2.53).

\section{d) Einfluss der Verfestigungseigenschaften auf $\Theta_{\mathrm{kr} \mathrm{e}}$}

Nebst der Gleichmassdehnung sind die Verfestigungseigenschaften, oder grob ausgedrückt, der Unterschied zwischen der nominellen Fliessspannung $\sigma_{f}$ und der Bruchfestigkeit $\beta_{z}$, ein hervorstechendes Merkmal des Spannungs-DehnungsDiagrammes der Zugarmierung. Dieser Unterschied wird gelegentlich auch durch das sogenannte Streckgrenzen-Verhältnis $\sigma_{s} / \beta_{z}$ charakterisiert. Auch diese Eigenschaften haben einen wesentlichen Einfluss auf den kritischen Gelenkwinkel infolge Versagen der Zugarmierung. Zur Verdeutlichung der Zusammenhänge werden wiederum die Formeln ( $90 \mathrm{a}),(80 \mathrm{a})$ und ( $79 \mathrm{a})$ benützt, indem bei gleichbleibender kritischer Stahldehnung $\varepsilon_{\text {e } r}=30 \%$ der Verfestigungsmodul $\mathrm{E}_{\mathrm{v}}$ von $20^{\prime} 000$ bis $50^{\prime} 000 \mathrm{~kg} / \mathrm{cm}^{2}$ variiert wird. Dabei nimmt allerdings die Bedingung ( 86 ) wieder recht ungünstige Werte an. Für $E_{v}=50^{\prime} 000 \mathrm{~kg} / \mathrm{cm}^{2}$ wird: 


$$
\frac{s}{d}=10,0
$$

Dies ist bei der Interpretation der Resultate zu berïcksichtigen.

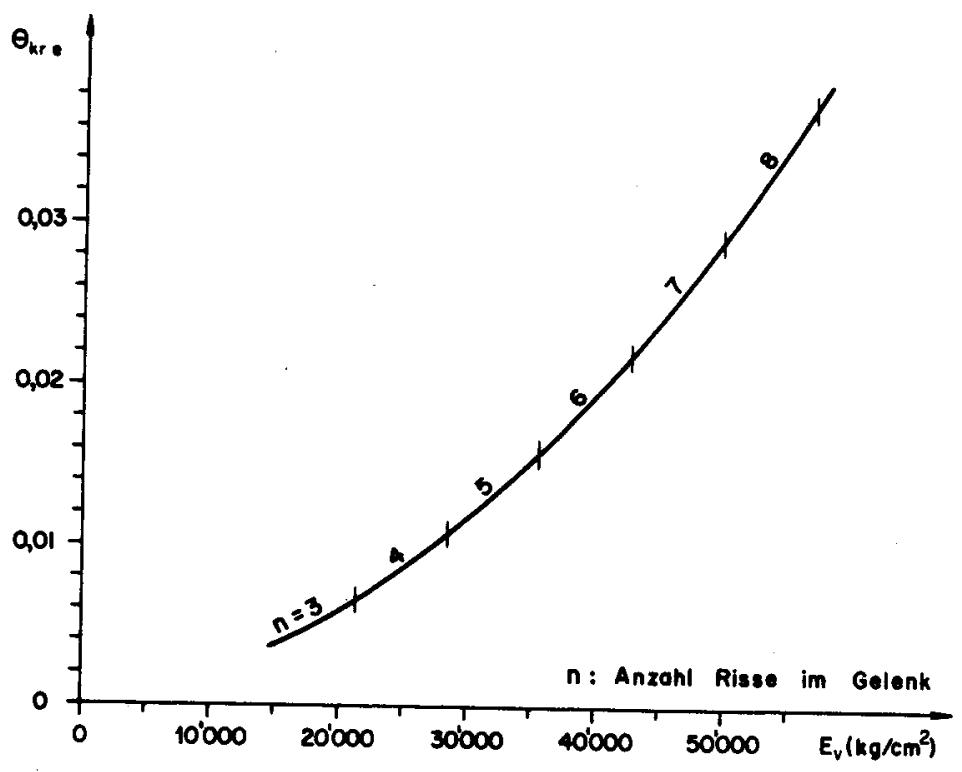

Bild 24

Einfluss der Verfestigung auf $\theta_{\mathrm{kr} \mathrm{e}}$ (Beispiel)

Das Ergebnis für die Zahlenwerte

$$
\begin{array}{lll}
\sigma_{f}=4000 \mathrm{~kg} / \mathrm{cm}^{2}, & \tau_{\mathrm{v}}^{*}=70 \mathrm{~kg} / \mathrm{cm}^{2}, & \varepsilon_{\mathrm{er}}=30 \% 0, \\
\varepsilon_{\mathrm{f}}=1,91 \% \circ, & \mathrm{Q} / \mathrm{Z}_{\mathrm{f}}=0,10, & \mathrm{~s} / \mathrm{y}=0,50 .
\end{array}
$$

ist in Bild 24 dargestellt. Es zeigt sich eine bedeutende Zunahme von $\theta_{\mathrm{kr}} \mathrm{e}$ mit wachsendem Verfestigungsmodul. Dadurch, dass im kritischen Riss bis 
zum Versagen des Stahles die Kraft stärker ansteigen muss, entstehen in mehr Rissen grössere plastische Defọrmationen der Zugarmierung. Für SpannungsDehnungs-Diagramme üblicher Stähle dürfte allerdings die Abhängigkeit nicht derart ausgeprägt sein, wie aus Bild 24 infolge der recht speziellen Annahmen und Grundlagen hervorgeht. Trotzdem zeigt sich eindrücklic $h$, wie wichtig die Verfestigungseigenschaften für die Grösse $\theta_{\mathrm{kr}} \mathrm{e}^{\text {sind. }}$

\section{e) Einfluss der Verbundeigenschaften auf $\theta_{\mathrm{kr} \mathrm{e}}$}

Die Güte des Verbundes beeinflusst ebenfalls den kritischen Gelenkwinkel infolge Versagen des Stahles. Im Ausdruck (90a) charakterisiert $\tau_{\mathrm{v}}^{*}$ die Verbundeigenschaften. Diese Gleichung wurde für einen festen Wert $s / y=0,5$ in Bild 25 gestrichelt dargestellt. Im übrigen liegen die folgenden Zahlenwerte zugrunde:

$$
\begin{aligned}
& G_{\mathrm{f}}=4000 \mathrm{~kg} / \mathrm{cm}^{2} \text {, } \\
& \mathrm{E}_{\mathrm{v}}=32^{\prime} 000 \mathrm{~kg} / \mathrm{cm}^{2}, \quad \varepsilon_{\mathrm{er}}=30 \% \text {, } \\
& \varepsilon_{f}=1,91 \% 0 \text {, } \\
& Q / Z_{f}=0,10 \text {, } \\
& d /(h-z)=0,06 \text {. }
\end{aligned}
$$

Die resultierende Kurve verläuft im Bereich $\tau_{v}^{*}=40 \div 100 \mathrm{~kg} / \mathrm{cm}^{2}$ recht steil. Die Abnahme von $\Theta_{\mathrm{kr}}$ mit grösserer Verbundspannung $\tau_{\mathrm{v}}^{*}$ ergibt sich vor allem deshalb, weil grössere Verbundspannungen die Spannungen in der Zugarmierung zwischen den Rissen stärker abbauen. Da im plastischen Bereich des SpannungsDehnungs-Diagrammes der Stahleinlagen verhältnismässig geringe Spannungsänderungen bedeutende Dehnungsänderungen zur Folge haben, bewirken grössere Werte $\tau_{\mathbf{v}}^{*}$ eine Abnhame des Verbundkoeffizienten $\kappa$, das heisst eine Reduktion der mittleren Stahldehnung.

Der tatsăchliche Einfluss der Verbundspannungen auf $\Theta_{\mathrm{kr}}$ dürfte jedoch etwas geringer sein als die Steilheit der gestrichelten Kurve in Bild 25 wiedergibt, da die Verbundeigenschaften den Rissabstand teilweise mitbestimmen. Wird in Gleichung ( 79a ) die Rissweite nach ( 63a ) eingesetzt, so ergibt sich:

$$
\Delta \varepsilon_{\text {emax }}=0,2 \cdot \frac{\sigma_{f}}{E_{v}} \cdot \frac{Q}{Z_{f}} \cdot \frac{d}{y} \cdot \frac{1}{\mu} \cdot \frac{b_{o}}{b} \cdot \frac{\sigma_{b z}}{\tau_{v R}}
$$




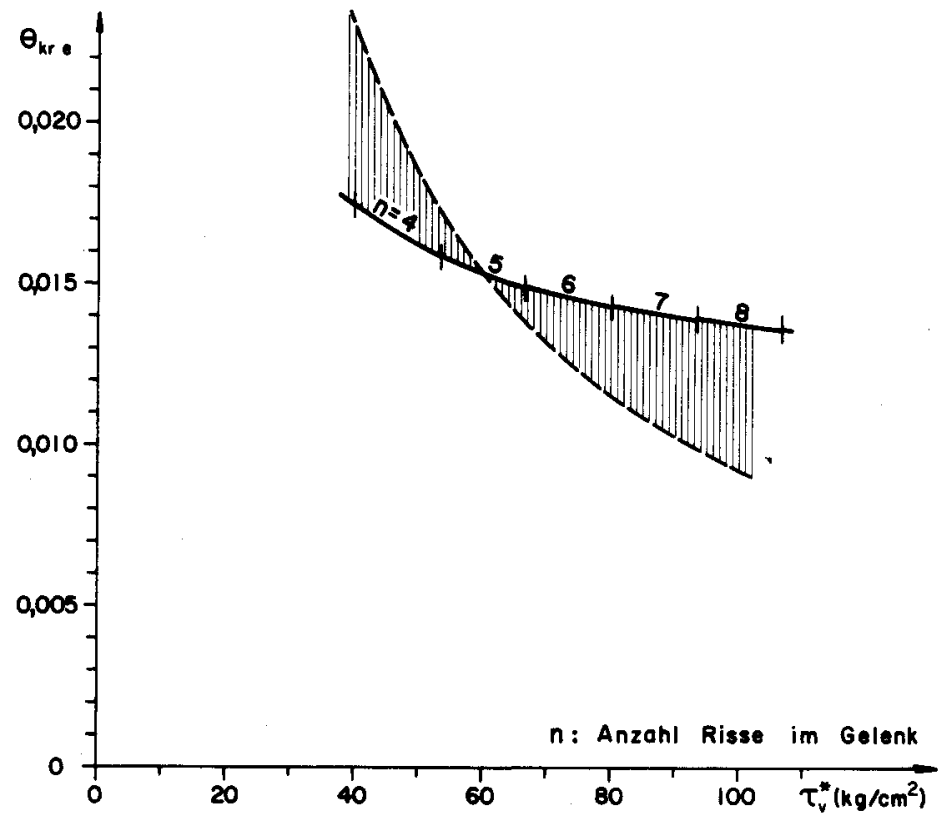

Bild 25

Einfluss der Verbundeigenschaften auf $\Theta_{\mathrm{kr} e}$ (Beispiel)

Mit den weiteren Zahlenwerten

$\mathrm{d} / \mathrm{y}=0,05, \mu=1 \%, \quad \mathrm{~b}_{\mathrm{o}} / \mathrm{b}=1, \quad \sigma_{\mathrm{bz}}=30 \mathrm{~kg} / \mathrm{cm}^{2}$,

erhălt man mit der Näherung $\tau_{v R} \cong \tau_{v}^{*}$ die Treppenkurve in Bild 25. Diese verlăuft weniger steil als die gestrichelte Kurve, da sie die Veränderung des Rissabstandes durch die variablen Verbundeigenschaften mitberücksichtigt. Trotzdem ist immer noch die eindeutige Tendenz zur Abnahme von $\Theta_{k r e}$ mit besserem Verbund zu erkennen.

Für den Rissabstand spielen jedoch besonders bei kleinen Armierungsgehalten auch andere Einflüsse eine Rolle, welche in ( $63 a$ ) nicht berücksichtigt werden 
(vergleiche 2.3). Diese vermindern die direkte Abhängigkeit des Rissabstandes von den Verbundspannungen. Es kann deshalb festgestellt werden, dass in Bild 25 der effektive Zusammenhang zwischen $\theta_{k r e}$ und den Verbundeigenschaften etwa durch den schraffierten Bereich dargestellt wird. Somit wird der kritische Rotationswinkel infolge Versagen der Zugarmierung umso kleiner, je besser der Verbund ist. Denn höhere Verbundspannungen bewirken eine stärkere Konzentration der Stahldehnungen auf die Risse zu, sodass die kritische Dehnung $\varepsilon_{\text {er }}$ bei einem kleineren Gelenkwinkel erreicht wird. Mit besseren Verbundeigenschaften erhöht sich auch die Gefahr eines Versagens der Zugarmierung vor demjenigen des Betons (vergleiche 2.53).

\section{f) Einfluss des Stabdurchmessers auf $\Theta_{\mathrm{kre}}$}

Zum Schluss sei noch kurz der Einfluss des Durchmessers der Zugarmierungsstäbe auf den kritischen Gelenkwinkel infolge Versagen des Stahls untersucht.

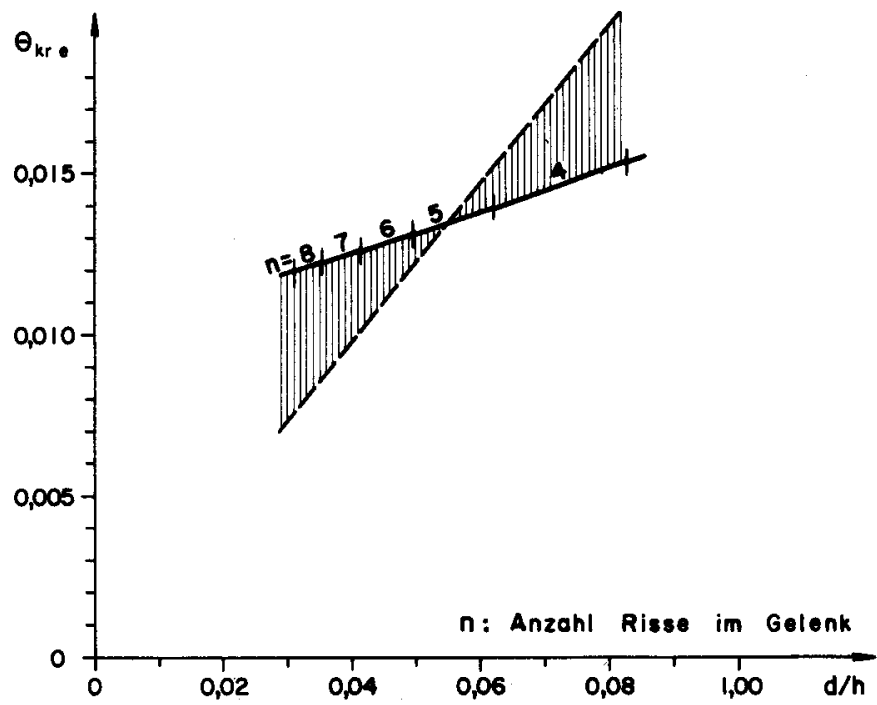

Bild 26

Einfluss des Stabdurchmessers auf $\Theta_{\mathrm{kr}} \mathrm{e}^{\text {(Beispiel) }}$ 
Werden die Ausdrücke ( $90 a)$ und ( $76 a$ ) beziehungsweise ( 94 ) etwas umgeformt, so kann $\Theta_{\mathrm{kre}}$ in Abhängigkeit vom Quotienten $\mathrm{d} / \mathrm{h}$ dargestellt werden. Mit den Zahlenwerten

$$
\begin{aligned}
& \sigma_{f}=4000 \mathrm{~kg} / \mathrm{cm}^{2}, \quad E_{v}=32,000 \mathrm{~kg} / \mathrm{cm}^{2}, \quad \varepsilon_{\mathrm{er}}=30 \% 0, \\
& \varepsilon_{\mathrm{f}}=1,91 \% 0, \quad Q / \mathrm{Z}_{\mathrm{f}}=0,10, \quad \mathrm{z} / \mathrm{h}=0,1, \quad \mathrm{y} / \mathrm{h}=0,95, \\
& \mu=1 \%, \quad \mathrm{~b} / \mathrm{b}_{\mathrm{o}}=1, \quad \sigma_{\mathrm{bz}}=30 \mathrm{~kg} / \mathrm{cm}^{2}, \quad \tau_{\mathrm{v}}^{*} \cong \tau_{\mathrm{vR}}=70 \mathrm{~kg} / \mathrm{cm}^{2},
\end{aligned}
$$

ergibt sich die Darstellung in Bild 26. Die gestrichelte Linie gilt wiederum für den Fall, wenn in Gleichung (79a) der Wert $s / y$ fest mit 0,5 angenommen wird. Die Treppenkurve hingegen resultiert bei Verwendung von Gleichung ( 94 ), das heisst wenn der Einfluss des Stabdurchmessers auf den Rissabstand nach (63a) berücksichtigt wird. Die tatsächlichen Zusammenhänge werden analog wie in Bild 25 etwa durch den schraffierten Zwischenbereich dargestellt. Für kleinere Armierungsgehalte dürfte eher die steilere Kurve zutreffen.

Man erkennt, dass mit grösserem Stabdurchmesser der kritische Gelenkwinkel infolge Versagen des Stahles anwächst. Die Zugkraft wird neben den Rissen weniger stark abgebaut, wodurch sich die mittlere Stahldehnung vergrössert. Kleinere Stabdurchmesser haben somit auf den kritischen Gelenkwinkel infolge Versagen der Zugarmierung eine ähnliche Wirkung wie verbesserte Verbundeigenschaften, was auch aus den benützten Gleichungen hervorgeht.

Die Untersuchungen des Abschnittes 2.51 zeigen, dass die Rotationsfähigkeit in Biegeriss-Gelenken von verschiedenen Parametern ganz erheblich beeinflusst wird. Der kritische Gelenkwinkel infolge Versagen des Betons nimmt insbesondere ab mit

- grösserer Querkraft,

- grösserem Rissabstand.

Der kritische Gelenkwinkel infolge Versagen der Zugarmierung wird kleiner mit 
- grösserer Querkraft,

- grösserem Rissabstand,

- kleinerer Gleichmassdehnung,

- schlechteren Verfestigungseigenschaften,

- besseren Verbundeigenschaften,

- kleineren Stabdurchmessern.

Soll zum Beispiel die Gefahr für ein Versagen des Stahles vor demjenigen des Betons beurteilt werden (vergleiche auch c) in 2.53), so muss der Einfluss all dieser Parameter berücksichtigt werden. Sind verschiedene Stahlsorten bezïglich dieser Gefahr miteinander zu vergleichen, so sind nebst den Dehnungseigenschaften auch die Verfestigungs- und Verbundeigenschaften in Betracht zu ziehen.

Interessant ist noch ein Vergleich mit der üblichen Konstruktionslehre von Stahlbetontragwerken. Dort werden im Hinblick auf den Gebrauchszustand (Rissebildung) möglichst gute Verbundeigenschaften sowie kleine Stabdurchmesser gefordert. Wie die vorangehenden Untersuchungen zeigen, wirken sich diese auf das Verformungsvermögen plastifizierter Bereiche jedoch nachteilig aus.

\subsection{Vergleiche mit Versuchsresultaten}

Bei den Versuchen über das plastische Verhalten von statisch unbestimmten Stahlbetonbalken [7] versagten die Balken A1, A2 und A3 im Feld, wo sich typische Biegeriss-Gelenke ausgebildet hatten. Der Bruch trat stets durch Einschnüren und Zerreissen der Zugarmierung ein. Die in den dortigen Messabschnitten festgestellten Winkel können mit denjenigen verglichen werden, die sich nach den hier hergeleiteten Formeln berechnen lassen.

Im folgenden werden nur die Resultate der Berechnungen aufgeführt. Im Anhang sind für alle drei Balken die benützten Grössen aus den Versuchen sowie als Beispiel der gesamte numerische Rechengang für den Balken A1 angegeben.

\section{a) Balken A1}

Im Bereich der grössten plastischen Deformationen, das heisst im Messabschnitt 19/20, befanden sich zwei Risse (vergleiche in [7] Figuren 20, 54 und 
Abbildung 3): Ein erster (kritischer) Riss, in welchem der Bruch erfolgte, und ein zweiter Riss.

\section{Rechnerischer Winkel}

Rechnerischer Winkel im ersten (kritischen) Riss infolge Versagen des Stahles:

$$
\omega_{1 \mathrm{krite}}=0,00423
$$

Rechnerischer Winkel im zweiten Riss bei Versagen des Stahles im ersten (kritischen) Riss:

$$
\omega_{2 \mathrm{e}} \quad=0,00178
$$

Rechnerischer kritischer Winkel im Messabschnitt 19/20 bei Versagen des Stahles:

$$
\omega_{19 / 20 \text { krite }}=\omega_{1 \text { krit e }}+\omega_{2 \mathrm{e}}=0,00601
$$

Versagen des Betons ist nicht massgebend (siehe Anhang).

\section{Gemessener Winkel}

Der in einem Versuchsbalken im plastischen Gelenk, beziehungsweise in einem Messabschnitt desselben, vorhandene Winkel beim Versagen des Stahles, welcher dem rechnerischen kritischen Winkel entspricht, ist schwierig festzustellen. Denn der Beginn der Einschnürung der Zugarmierung (Erreichen der Gleichmassdehnung) im kritischen Riss kann messtechnisch kaum erfasst werden. Wohl tritt schliesslich eine Abnahme des im plastischen Gelenk übertragenen Biegemomentes ein. Vom Ueberschreiten der Gleichmassdehnung im Riss bis zum Erreichen des maximalen Biegemomentes kann jedoch die Breite des kritischen Risses unter Umständen noch wesentlich zunehmen. Denn unmittelbar nach Beginn der Einschnürung wird die Zugkraft vorerst noch kaum reduziert. Durch die meist starke Zunahme der Rissbreite können hingegen der innere Hebelarm und damit das Biegemoment noch anwachsen. Auch ist es möglich, dass in dieser Phase durch die sehr grossen Verschiebungen zwischen dem 
Beton und der Zugarmierung, sowie durch die plastische Querkontraktion der Zugstäbe, am Rissrand die Verzahnung des Betonmörtels mit den Stahlstäben ein Stück weit gelockert wird. Dadurch können die dort wirkenden Verbundspannungen reduziert werden, sodass im Vergleich zur theoretischen Berechnung grössere Deformationen entstehen. Nach fortgeschrittener Einschnürung tritt bestimmt eine solche Abnahme der Verbundspannungen ein, da sich die Stahlstäbe durch die Verringerung ihres Durchmessers gewissermassen dem Beton entziehen.

Aufgrund der vorstehenden Ueberlegungen wird im Sinne einer einfachen und natürlich näherungsweisen Regelung als "gemessener" kritischer Winkel der bei der Laststufe vor dem Erreichen des maximalen Momentes vorhandene Winkel benüitzt.

Der gemessene kritische Winkel beträgt beim Balken A1 im Messabschnitt 19/ 20 bei Laststufe 14 (vergleiche Tabelle 9 und Figur 46 in [7] ):

$$
\omega_{19 / 20 \text { krite }}=0,00607
$$

Dieser Winkel stimmt zufälligerweise mit dem berechneten fast überein.

\section{b) Balken A2}

Im Balken A2 traten die grössten plastischen Deformationen ebenfalls im Messabschnitt 19/20 auf, wo sich wiederum zwei Risse befanden.

\section{Rechnerischer Winkel}

Rechnerischer Winkel im ersten (kritischen) Riss infolge Versagen des Stahles:

$$
\omega_{1 \text { krite }}=0,00463
$$

Dieser Winkel im kritischen Riss ist also etwa derselbe wie im Balken A1.

Rechnerischer Winkel im zweiten Riss bei Versagen des Stahles im ersten (kritischen) Riss: 
$\omega_{2 \mathrm{e}}=0,00251$

Der Winkel im zweiten Riss ist hier grösser als im Balken A1, was auf den Einfluss der Querkraft zurückzuführen ist. Diese ist kleiner als im Balken A1. Dadurch wird im Balken A2 der Unterschied zwischen den Zugkräften im ersten (kritischen) und im zweiten Riss geringer, womit im zweiten Riss auch die plastischen Deformationen der Zugarmierung grösser ausfallen. Dieses Ergebnis entspricht den Erkenntnissen von Abschnitt 2.51 a) mit Bild 21.

Rechnerischer kritischer Winkel im Messabschnitt 19/20 bei Versagen des Stahles:

$$
\omega_{19 / 20 \text { krite }}=0,00714
$$

Versagen des Betons ist hier ebenfalls nicht massgebend.

\section{Gemessener Winkel.}

Der gemessene kritische Winkel beim Balken A2 beträgt bei Laststufe 16 im Messabschnitt 19/20 (vergleiche Tabelle 9 und Figur 46 in [7] ):

$$
\omega_{19 / 20 \text { krite }}=0,01120
$$

Die Uebereinstimmung mit dem gerechneten Wert ist hier nicht so gut wie beim Balken A1. Immerhin befindet sich der rechnerische Wert auf der sicheren Seite. Auch war beim Balken A2 der zweite Riss im Verhältnis zum ersten tatsächlich breiter als im Balken A1 (vergleiche Figuren 54 und 55 in [7] ). Dies bedeutet, dass der Einfluss der Querkraft rechnerisch richtig erfasst wird.

\section{c) Balken A3}

Im Balken A3 war das Feldmoment im Messbereich 16/20 in den höheren Laststufen nahezu konstant. Die grössten Deformationen und schliesslich der Bruch traten im Messabschnitt 18/19 auf, in welchem sich zwei Risse befanden. 


\section{Rechnerischer Winkel}

Rechnerischer Winkel im ersten (kritischen) Riss infolge Versagen des Stahles:

Es ergibt sich derselbe Winkel wie im kritischen Riss des Balkens A2:

$$
\omega_{1 \mathrm{krite}}=0,00463
$$

Rechnerischer Winkel im zweiten Riss bei Versagen des Stahles im ersten (kritischen) Riss:

Da die Querkraft klein war, ist die Stahlspannung nur $24 \mathrm{~kg} / \mathrm{cm}^{2}$ geringer als im kritischen Riss. Der Winkel im zweiten Riss ist somit praktisch derselbe wie im kritischen Riss:

$$
\omega_{2 \mathrm{e}}=0,00463
$$

Rechnerischer kritischer Winkel im Messabschnitt 18/19 bei Versagen des Stahles:

$$
\omega_{18 / 19 \text { krit e }}=0,00926
$$

Versagen des Betons ist wiederum nicht massgebend.

\section{Gemessener Winkel}

Der Winkel im Messabschnitt 19/20 betrug vor dem Erreichen des maximalen Momentes, das heisst bei Laststufe 27 (vergleiche Tabelle 9 und Figur 47 in [7] ):

$$
\omega_{18 / 19 \text { krite }}=0,01540
$$

Dieser Wert ist - ähnlich wie beim Balken A2 - grösser als der rechnerische Wert. Dazu mag teilweise die Tatsache beitragen, dass im Messabschnitt 18/ 19 noch zwei weitere, ca. $8 \mathrm{~cm}$ lange Risse vorhanden waren. Solche Risse verändern den theoretisch angesetzten Verlauf der Verbundspannungen und Stahldehnungen zwischen den Hauptrissen und können damit zum kritischen Win- 
kel einen gewissen Beitrag leisten.

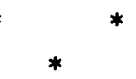

Die Vergleiche zwischen Versuchsresultaten und rechnerischen Werten für den kritischen Winkel bei Versagen des Stahles zeigen beim Balken A1 fast vollkommene Uebereinstimmung. Bei den Balken A2 und A3 hingegen betragen die rechnerischen Werte nur $64 \%$ beziehungsweise $60 \%$ der gemessenen. Diese unterschiedliche Uebereinstimmung hängt unter anderem damit zusammen, dass der "gemessene" kritische Winkel nur sehr schlecht und unsicher bestimmt werden kann. Bei stetig gesteigerter Belastung wäre das Moment über einen ausserordentlich grossen Bereich des zunehmenden Winkels nahezu konstant. Im Belastungsverfahren des Versuches (vergleiche [7] Seite 28) hängt die Endlast einer Laststufe und damit das zugehörige Moment unter anderem von der Belastungsgeschwindigkeit, der Anfangslast und vor allem der Messdauer ab. Es können somit Zufälligkeiten die Laststufe mit dem maximalen Moment beziehungsweise die vorangehende Laststufe und damit den "gemessenen" kritischen Winkel sehr stark beeinflussen.

Immerhin zeigen die Vergleiche, dass mit den entwickelten theoretischen Beziehungen der kritische Winkel verhältnismässig zutreffend berechnet werden kann. Die Theorie erfasst die tatsächlichen Verhältnisse grundsätzlich richtig. Im Hinblick auf die im Abschnitt 2.52 a) angefuihrten Effekte, welche den kritischen Winkel vergrössern, in der Rechnung aber nicht berücksichtigt werden, liegen die theoretischen Werte stets auf der sicheren Seite. Es kann somit festgehalten werden:

Die aus der theoretischen Berechnung resultierenden Werte für den kritischen Gelenkwinkel in einem Biegeriss-Gelenk stellen einen sicheren unteren Grenzwert dar.

\subsection{Vergleiche mit üblicher Betrachtungsweise}

In der Stahlbetontheorie ist es allgemein üblich, die Hypothese vom Ebenbleiben der Querschnitte vorauszusetzen. Auch verschiedene Formeln für den "möglichen" oder "erlaubten" Rotationswinkel in plastischen Stahlbetongelenken, welcher un- 
serem kritischen Gelenkwinkel entspricht, basieren direkt oder indirekt auf dieser Annahme (zum Beispiel [14] , [20] ). Dabei wird, meist getrennt für Zug- und Druckzone, wie bei homogenen isotropen Baustoffen, zuerst eine Bruchkrümmung berechnet und diese dann anschliessend über eine bestimmte "Gelenklänge" integriert beziehungsweise mit derselben multipliziert. Für die Gelenklänge werden verschiedene Formeln angegeben.

Bei der Herleitung der vorstehend entwickelten Beziehungen wurde auf die Hypothese vom Ebenbleiben der Querschnitte bewusst verzichtet und versucht, die tatsächlichen Verhältnisse möglichst zutreffend zu erfassen. Es ist daher von Interesse, die Ergebnisse der beiden Betrachtungsweisen in einigen Punkten miteinander $\mathrm{zu}$ vergleichen.

Dieser Vergleich hat den Bereich des einzelnen, das heisst des kritischen Biegeriss-Elementes, zur Grundlage. Dieses sei ausschliesslich durch ein Biegemoment beansprucht. Bei Betrachtung mehrerer Biegeriss-Elemente beziehungsweise eines ganzen Gelenkes müsste der Einfluss einer Querkraft miteinbezogen werden. Dadurch könnten jedoch keine geschlossenen Ausdrücke mehr hergeleitet werden, was bei Beschränkung auf ein einzelnes Biegeriss-Element mit einigen vereinfachenden Annahmen noch möglich ist. Ausserdem wurde in 2.51 der Einfluss einer Querkraft bereits näher untersucht.

Vorerst sollen die kritischen Elementwinkel für Versagen des Betons beziehungsweise des Stahles nach beiden Betrachtungsweisen verglichen werden. Anschliessend werden die Armierungsgehalte für gleichzeitiges Versagen der Zugarmierung und des Betons sowie für Sprödbrüche diskutiert.

\section{a) Vergleich von $\omega_{\mathrm{kr} \mathrm{b}}$}

Nach der üblichen Betrachtungsweise wird die Krümmung beim Versagen des Betons:

$$
\mathrm{k}_{\mathrm{krb}}=\frac{\varepsilon_{\mathrm{b} \mathbf{r}}}{\mathrm{z}}
$$

$\varepsilon_{\mathrm{br}}$ ist die Betonbruchstauchung am Druckrand. $\mathrm{z}$ bedeutet hier nicht den Abstand des Rissendes sondern denjenigen einer fiktiven "neutralen Axe" von der Druckkante. $z$ kann entweder ausschliesslich aus einer Gleichgewichtsbedingung 
unter Annahme von Grösse und Verteilung der Betonspannungen oder auch mit Hilfe einer zusätzlichen Verträglichkeitsbedingung zusammen mit Annahmen über die Formänderungseigenschaften der Baustoffe bestimmt werden (zum Beispiel Ebenbleiben der Querschnitte, Hooke'sches Gesetz, n-Theorie, und so weiter). Einfachheitshalber wird hier $z$ nach ( 5 ) für $\mu^{\prime}=0$ verwendet. Die Differenz zwischen $z$ nach ( 5 ) und derselben Grösse aus einem der obigen Verfahren ist gering und für die hiesigen Ergebnisse unerheblich. Damit wird aus ( 95 ) der Winkel innerhalb eines Biegeriss-Elementes beim Versagen des Betons:

$$
\omega_{\mathrm{krb}}=\mathrm{k}_{\mathrm{krb}} \cdot \mathrm{s}=\varepsilon_{\mathrm{b} r} \cdot \frac{0,8 \beta}{\sigma_{\mathrm{f}}} \cdot \frac{\mathrm{s}}{\mathrm{h}} \cdot \frac{1}{\mu}
$$

Anderseits ergibt sich mit $\varepsilon_{b \max }=\varepsilon_{b r}$ aus ( 48 ) mit (46a) beziehungsweise ( 47 ):

$$
\begin{array}{ll}
\frac{z}{s}=1,0: & \omega_{k r b}=\frac{\varepsilon_{b r}}{\left[\frac{z}{s}\right]^{1 / 3}} \\
\frac{z}{s}=1,0: & \omega_{k r b}=\frac{\varepsilon_{b r}}{\left[\frac{z}{s}\right]^{1}}
\end{array}
$$

Mit 2 nach ( 5 ) wird für $\mu^{\prime}=0$ :

$$
\begin{array}{ll}
\frac{z}{s}=1,0: & \omega_{k r b}=\frac{\varepsilon_{b r}}{\left[\frac{\sigma_{f}}{0,8 \beta} \cdot \frac{h}{s} \cdot \mu\right]^{1 / 3}} \\
\frac{z}{s}=1,0: & \omega_{k r b}=\frac{\varepsilon_{b r}}{\left[\frac{\sigma_{f}}{0,8 \beta} \cdot \frac{h}{s} \cdot \mu\right]^{T}}
\end{array}
$$


Man erkennt, dass Gleichung ( 100 ) identisch ist mit Gleichung ( 96 ). Tatsăchlich sind für $\mathrm{z} / \mathrm{s}>1,0$ die Risse relativ eng beieinander, respektive der $\mathrm{Ab}-$ stand der Rissenden von der Druckkante ist recht gross im Verhältnis zum Rissabstand. Die Variation der Betonrandstauchung ist deshalb vernachlässigbar gering, und eine Mittelwertbildung, wie sie die Annahme vom Ebenbleiben der Querschnitte darstellt, erscheint als zulässig. Für $z / s<1,0$ können jedoch mit ( 96 ) die tatsächlichen Verhältnisse nicht mehr zutreffend erfasst werden.

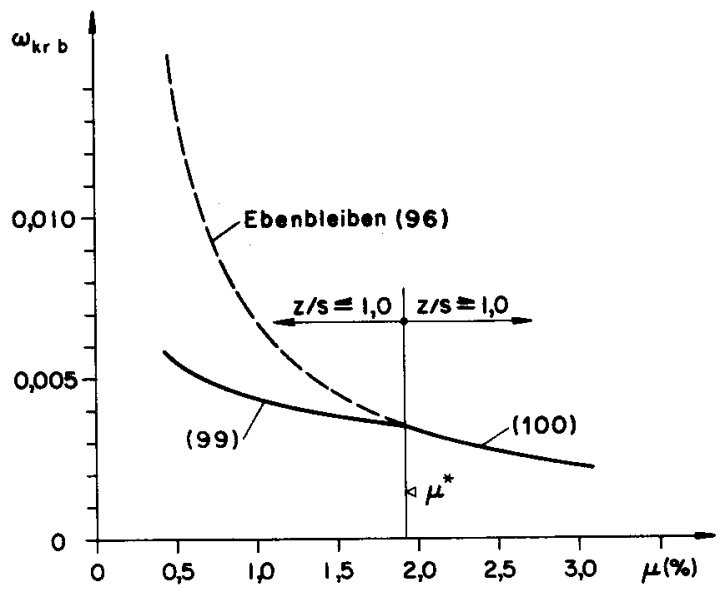

Bild 27

Abhängigkeit des kritischen Elementwinkels infolge Versagen des Betons vom Zugarmierungsgehalt für verschiedene Annahmen (Beispiel)

Interessant ist der Vergleich über den Einfluss des Zugarmierungsgehaltes auf den kritischen Elementwinkel. In Bild 27 ist $\omega_{\mathrm{krb}}$ nach den Gleichungen ( 96 ), ( 99 ) und ( 100$)$ in Abhängigkeit von $\mu$ aufgetragen worden. Dabei wurden die folgenden Zahlenwerte angenommen: 


$$
\begin{aligned}
& \varepsilon_{\mathrm{br}}=3,5 \% \mathrm{o}, \quad \sigma_{\mathrm{f}}=4000 \mathrm{~kg} / \mathrm{cm}^{2}, \quad \beta_{\mathrm{w}}=400 \mathrm{~kg} / \mathrm{cm}^{2}, \\
& \beta=320 \mathrm{~kg} / \mathrm{cm}^{2}, \\
& \mathrm{~s} / \mathrm{h}=0,3 .
\end{aligned}
$$

Der Armierungsgehalt $\mu^{*}$, für welchen $z / s=1,0$ wird, respektive die Gleichungen ( 99 ) und ( 100$)$ identisch sind, beträgt:

$$
\mu^{*}=\frac{0,8 \beta}{\sigma_{f}} \cdot \frac{s}{h}
$$

Für die obigen Zahlenwerte ergibt sich $\mu^{*}=1,92 \%$.

Bild 27 zeigt, dass bei abnehmendem Armierungsgehalt der kritsiche Elementwinkel längst nicht derart zunimmt, wie sich aufgrund der Hypothese vom Ebenbleiben der Querschnitte ergibt. Da der Abstand des Rissendes von der Druckkante klein ist gegenüber dem Rissabstand, konzentrieren sich die Betonrandstauchungen stärker auf die Rissegegend. Die Bruchstauchung wird daher bei einem kleineren Elementwinkel erreicht als man aufgrund der Annahme vom Ebenbleiben der Querschnitte erwarten würde.

\section{b) Vergleich von $\omega$}

Die Krümmung beim Versagen der Zugarmierung beträgt nach der üblichen Betrachtungsweise:

$$
k_{k r e}=\frac{\varepsilon_{e r}}{h-z}
$$

$\varepsilon_{\text {er }}$ bedeutet die Stahldehnung beim Beginn der Einschnürung. z kann entsprechend Gleichung ( $5 a$ ) für $\mu^{\prime}=0$ eingesetzt werden. Damit wird der Winkel innerhalb eines Biegeriss-Elementes beim Versagen des Stahles:

$$
\omega_{k r e}=k_{k r e} \cdot s=\frac{\varepsilon_{e r}}{1-\frac{\beta_{z} \cdot \mu}{0,8 \beta}} \cdot \frac{s}{h}
$$


Anderseits ergibt sich aus ( 38 ):

$$
\omega_{\text {kre }}=\frac{\varepsilon_{\text {er }} \cdot \kappa}{1-\frac{\beta_{\mathrm{z}}{ }^{\mu}}{0,8 \beta}} \cdot \frac{s}{h}
$$

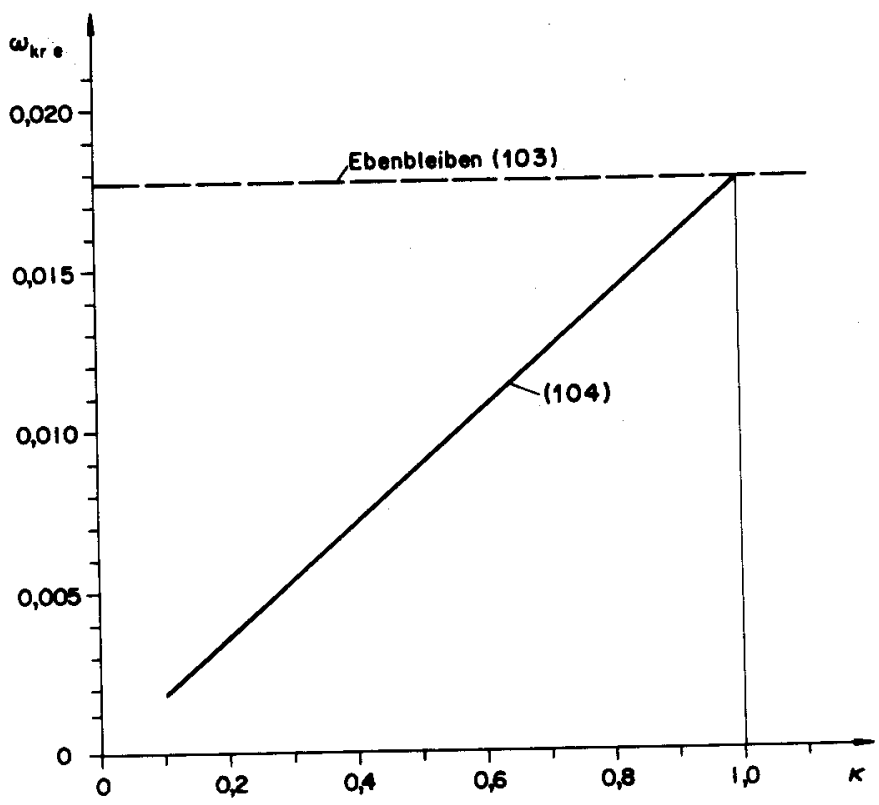

Bild 28

Kritischer Elementwinkel beim Versagen der Zugarmierung für verschiedene Annahmen (Beispiel)

In Bild 28 sind die Winkel $\omega_{k r}$ e nach den Gleichungen ( 103$)$ und ( 104$)$ in $\mathrm{Ab}-$ hängigkeit vom Verbundkoeffizienten graphisch dargestellt. Dazu wurden die folgenden Zahlenwerte verwendet:

$$
\varepsilon_{\text {er }}=5 \%, \quad \beta_{z}=5000 \mathrm{~kg} / \mathrm{cm}^{2}, \quad \beta_{w}=400 \mathrm{~kg} / \mathrm{cm}^{2},
$$




$$
\beta=320 \mathrm{~kg} / \mathrm{cm}^{2}, \quad \mu=1 \%, \quad \mathrm{~s} / \mathrm{h}=0,3 .
$$

Es ist offensichtlich, dass die Berechnung des kritischen Elementwinkels für Versagen der Zugarmierung aufgrund der Hypothese vom Ebenbleiben der Querschnitte zu hohe Werte ergibt, da sie die Reduktion der Stahlspannungen und -Dehnungen durch die Verbundspannungen zwischen den Rissen nicht berücksichtigt. Der Verbundkoeffizient $\kappa$, welcher diese Reduktion erfasst, kann beim Erreichen von $\beta_{\mathrm{z}}$ beziehungsweise $\varepsilon_{\mathrm{er}} \cong \lambda_{\mathrm{Gl}}$ je nach den Verhältnissen, ins besondere je nach Form des Spannungs-Dehnungs-Diagrammes und des Verbundgesetzes, Werte von nur 0,1 bis 0,2 annehmen. Dadurch wird $\omega_{\mathrm{kr}}$ nach ( 103 ) um diesen Faktor vermindert.

Die grundsätzli che Richtigkeit der Formel ( 104 ) beziehungsweise der vorangehend dargelegten Berechmungsmethode zeigt auch ein Vergleich mit Versuchsresultaten. In der folgenden Aufstellung sind die kritischen Winkel für Versagen der Zugarmierung aufgeführt, welche in den entsprechenden Messabschnitten der Balken A1, A2 und A3 der Versuche [7] berechnet beziehungsweise gemessen wurden. Die Zahlenwerte der ersten Spalte wurden nach der Formel ( 103 ) mit $\varepsilon_{\text {er }} \cong \lambda_{G l}$ ermittelt. Um den Vergleich mit den übrigen Spalten zu ermöglichen, musste der Rissabstand gleich der Länge des Messabschnittes (25 cm) gesetzt werden. Die Werte der zweiten und dritten Spalte wurden dem Abschnitt 2.52 entnommen.

\begin{tabular}{|c|c|c|c|c|}
\hline \multirow{2}{*}{ Balken } & \multirow{2}{*}{ Messabschnitt } & \multicolumn{3}{|c|}{ Krit. Winkel für Versagen der Zugarmierung } \\
\cline { 3 - 5 } & & $\begin{array}{l}\text { Berechnet } \\
\text { (Ebenbleiben) }\end{array}$ & $\begin{array}{l}\text { Berechnet } \\
\text { (aus 2.52) }\end{array}$ & Gemessen \\
\hline A1 & $19 / 20$ & 0,04310 & 0,00601 & 0,00607 \\
A2 & $19 / 20$ & 0,05070 & 0,00714 & 0,01120 \\
A3 & $18 / 19$ & 0,05070 & 0,00926 & 0,01540 \\
\hline
\end{tabular}

Man erkennt, dass die gemessenen Werte wohl über den rechnerischen aus 2.52 liegen. Sie erreichen jedoch niemals die hohen Werte, welche sich aus der Berechnung 
aufgrund der Hypothese vom Ebenbleiben der Querschnitte ergeben. Letztere sind ca. 3 bis 7 mal grösser als die gemessenen.

\section{c) Vergleich von $\mu_{\mathbf{k r}}$}

Gelegentlich wird die Gefahr für ein Versagen der Zugarmierung vor demjenigen des Betons durch den sogenannten kritischen Armierungsgehalt $\mu_{\mathbf{k r}}$ charakterisiert ( zum Beispiel [7] ). $\mu_{k r}$ bedeutet den Zugarmierungsgehalt, bei welchem Stahl und Beton gleichzeitig versagen. Somit gilt:

$\begin{array}{ll}\text { Versagen des Stahles : } & \mu \leq \mu_{k r} \\ \text { Versagen des Betons : } & \mu \geq \mu_{k r}\end{array}$

Nimmt man Ebenbleiben der Querschnitte an, so ergibt sich $\mu_{\mathbf{k r}}$ aus der Gleichsetzung von ( 96 ) mit ( 103 ), wobei in ( 96 ) anstelle von $\sigma_{f}$ nun $\beta_{z}$ zu setzen ist:

$$
\mu_{k r}=\frac{0,8 \beta}{\beta_{z}} \cdot \frac{\varepsilon_{b r}}{\varepsilon_{e r}+\varepsilon_{b r}}
$$

Anderseits sind je nach Grösse von $\mathrm{z} / \mathrm{s}$ zwei Fălle zu unterscheiden: Für $z / s \leq 1,0$ ergibt sich aus ( 97 ) mit ( 38 ):

$\frac{z}{s} \leq 1,0:\left(\frac{z}{h}\right) \cdot \varepsilon_{b r}+\left(\frac{z}{h}\right)^{1 / 3} \cdot\left(\frac{s}{h}\right)^{2 / 3} \cdot \kappa \cdot \varepsilon_{e r}=\varepsilon_{b r}$

Darin ist entsprechend ( $5 a$ ) für $\mu^{\prime}=0$ :

$$
\frac{z}{h}=\frac{\beta_{z}}{0,8 \beta} \cdot \mu_{k r}
$$

Das Verhältnis $s / h$ kann - da es sich um kleinere Werte $\mu$ handelt - als konstant und unabhängig von $\mu$ angenommen werden (vergleiche 2.3). 
Damit wird aus ( 106 ):

$\frac{z}{s}=1,0: \frac{\beta_{z} \cdot \varepsilon_{b r}}{0,8 \beta} \cdot \mu_{k r}+\left[\frac{\beta_{z}}{0,8 \beta}\right]^{1 / 3} \cdot \mu_{k r}^{1 / 3} \cdot\left(\frac{s}{h}\right)^{2 / 3} \cdot \kappa \cdot \varepsilon_{e r}=\varepsilon_{b r}$

Dieser Ausdruck stellt eine Gleichung für $\mu_{\mathbf{k r}}$ dar, welche am besten durch Probieren aufgelöst wird.

Für $z / s \geq 1,0$ wird aus der Gleichsetzung von ( 98 ) mit ( 38 ):

$\frac{\mathrm{z}}{\mathrm{s}} \geq 1,0: \quad \mu_{\mathrm{kr}}=\frac{0,8 \beta}{\beta_{\mathrm{z}}} \cdot \frac{\varepsilon_{\mathrm{br}}}{\varepsilon_{\mathrm{er} \mathbf{r}^{* \kappa}+\varepsilon_{\mathrm{br}}}}$

Dieser Ausdruck ist bis auf $\kappa$ im Nenner identisch mit Gleichung ( 105 ), welche auf der Annahme vom Ebenbleiben der Querschnitte beruht.

Die Gültigkeitsgrenze $\mu_{\mathrm{kr}}^{*}$ für ( 108 ) und ( 109 ) ergibt sich mit $\mathrm{z} / \mathrm{s}=1,0$ wie folgt:

$$
\mu_{\mathbf{k r}}^{*}=\frac{s}{h} \cdot \frac{0,8 \beta}{\beta_{z}}
$$

Das zugehörige $\kappa^{*}$ erhält man aus $(109)$ für $\mu_{\mathbf{k r}}=\mu_{\mathbf{k r}}^{*}$ :

$$
\kappa^{*}=\frac{\varepsilon_{b r}}{\varepsilon_{e r}} \cdot\left[\frac{0,8 \beta}{\beta_{z} \mu_{k r}^{*}}-1\right]
$$

Zum Vergleich ist in Bild 29 der Verlauf von $\mu_{k r}$ aus den Formeln (.105), ( 108 ) und ( 109 ) in Abhängigkeit vom Verbundkoeffizienten $\kappa$ dargestellt worden. Dazu wurden die folgenden Zahlenwerte angenommen:

$$
\begin{aligned}
& \varepsilon_{\mathrm{er}}=5 \%, \quad \varepsilon_{\mathrm{br}}=3,5 \% 0, \quad \beta_{\mathrm{z}}=5000 \mathrm{~kg} / \mathrm{cm}^{2}, \\
& \beta_{\mathrm{w}}=400 \mathrm{~kg} / \mathrm{cm}^{2}, \quad \beta=320 \mathrm{~kg} / \mathrm{cm}^{2}, \quad s / \mathrm{h}=0,2 .
\end{aligned}
$$




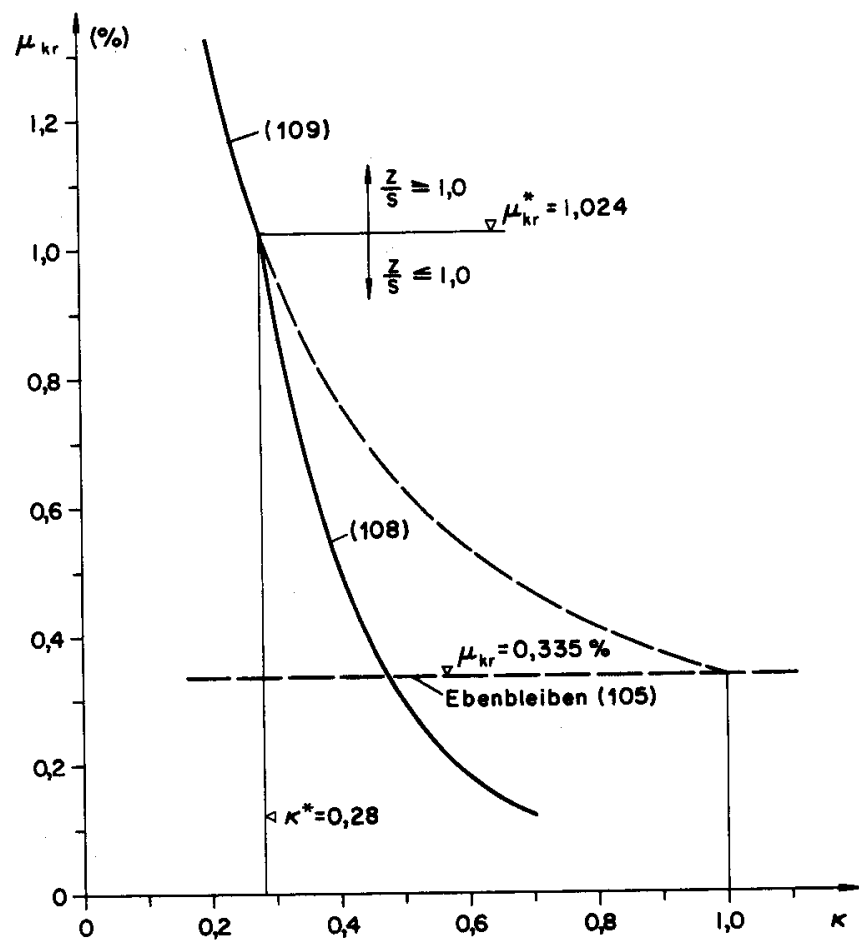

Bild 29

Armierungsgehalt für gleichzeitiges Versagen von Zugarmierung und Beton in Abhängigkeit vom Verbundkoeffizienten (Beispiel)

Bild 29 zeigt, wie der kritische Armierungsgehalt ausserordentlich stark von der Grösse des Verbundkoeffizienten abhängen kann. Insbesondere bei Armierungsgehalten $\mu<1,0 \%$, das heisst bei relativ grossem Rissabstand, treten bei hochwertigen Armierungsstählen mit guten Verbundeigenschaften oft kleine Verbundkoeffizienten auf. In solchen Fällen wird durch die ubliche Betrachtungsweise die Gefahr eines Versagens der Zugarmierung vor demjenigen des Betons ganz erheblich unterschätzt. 


\section{d) Vergleich von $\mu_{\mathrm{G}} \mathbf{r}$}

Eine weitere wichtige Kenngrösse in Stahlbeton-Biegebalken ist der sogenannte Grenzarmierungsgehalt $\mu_{\mathrm{Gr}}$. Dieser charakterisiert die Sprödbruchgefahr (vergleiche [2], [7] ). $\mu_{G r}$ bedeutet denjenigen Zugarmierungsgehalt, bei welchem gleichzeitig Versagen des Betons und "Fliessbeginn" in der Längsarmierung eintritt. Demnach gilt:

$$
\begin{array}{ll}
\text { Versagen des Betons vor Fliessbeginn: } \mu \geq \mu_{G r} \\
\text { Versagen des Betons nach Fliessbeginn: } \mu \leqslant \mu_{G r}
\end{array}
$$

Der Fliessbeginn soll mit der nominellen Fliessdehnung beziehungsweise der nominellen Fliessspannung $\varepsilon_{f}$ entsprechend Bild 2 definiert werden.

Wird Ebenbleiben der Querschnitte angenommen, so ergibt sich der Grenzarmierungsgehalt aus der Gleichsetzung von ( 94 ) mit ( 102 ), wobei jedoch anstelle von $\varepsilon_{\text {er }}$ die Fliessdehnung $\varepsilon_{f}$ zu setzen ist. Mit $(5)$ wird für $\mu^{\prime}=0$ :

$$
\mu_{G r}=\frac{0,8 \beta}{\sigma_{f}} \cdot \frac{\varepsilon_{b r}}{\varepsilon_{f}+\varepsilon_{b r}}
$$

Anderseits müssen je nach Grösse von $z / s$ wiederum $z$ wei Fälle unterschieden werden:

Für $z / s \leq 1,0$ ergibt sich analog zu ( 106 ):

$\frac{z}{s} \leq 1,0: \quad\left(\frac{z}{h}\right) \cdot \varepsilon_{b r}+\left(\frac{z}{h}\right)^{1 / 3} \cdot\left(\frac{s}{h}\right)^{2 / 3} \cdot \kappa \cdot \varepsilon_{f}=\varepsilon_{b r}$

Darin ist entsprechend ( 5 ) für $\mu^{\prime}=0$ :

$$
\frac{z}{h}=\frac{G_{f}}{0,8 \beta} \cdot \mu_{G r}=c_{1} \cdot \mu_{G r}
$$

Da der Armierungsgehalt $\mu_{G r}$ stets recht gross ist, wird noch dessen Ein- 
fluss auf den Rissabstand mittels ( 63a ) berücksichtigt:

$$
\frac{\mathrm{s}}{\mathrm{h}}=0,2 \frac{\mathrm{d}}{\mathrm{h}} \cdot \frac{\mathrm{b}_{\mathrm{o}}}{\mathrm{b}} \cdot \frac{\sigma_{\mathrm{bz}}}{\tau_{\mathrm{vr}}} \cdot \frac{1}{\mu_{\mathrm{Gr}}}=c_{2} \cdot \frac{1}{\mu_{\mathrm{Gr}}}
$$

Damit wird aus ( 113 ):

$\frac{z}{s} \leq 1,0: \quad c_{1} \cdot \varepsilon_{b r} \cdot \mu_{G r}+\left(c_{1} \cdot \mu_{G r}\right)^{1 / 3} \cdot\left(\frac{c_{2}}{\mu_{G r}}\right) \cdot \kappa \cdot \varepsilon_{f}=\varepsilon_{b r}$

Für $z / s=1,0$ erhält man eine zu ( 109 ) analoge Formel, wobei jedoch $\sigma_{f}$ anstelle von $\beta_{\mathrm{z}}$ und $\varepsilon_{\mathrm{f}}$ anstatt $\varepsilon_{\mathrm{er}}$ steht:

$\frac{z}{s}=1,0: \quad \mu_{G r}=\frac{0,8 \beta}{\sigma_{f}} \cdot \frac{\varepsilon_{b r}}{\varepsilon_{f}{ }^{k}+\varepsilon_{b r}}$

Die Giiltigkeitsgrenze $\mu_{\mathrm{Gr}}^{*}$ für (116) und (117) ergibt sich mit $\mathrm{z} / \mathrm{s}=1,0$ wie folgt:

$$
\mu_{G r}^{*}=\sqrt{\frac{c_{2}}{c_{1}}}=\sqrt{0,2 \frac{d}{h} \cdot \frac{b o}{b} \cdot \frac{\sigma_{b z}}{\tau_{v r}} \cdot \frac{0,8 \beta}{\sigma_{f}}}
$$

Die Zusammenhänge sind in Bild 30 für die folgenden Zahlenwerte dargestellt:

$$
\begin{aligned}
& \varepsilon_{\mathrm{br}}=3,5 \% \mathrm{o}, \quad \sigma_{\mathrm{f}}=4000 \mathrm{~kg} / \mathrm{cm}^{2}, \quad \varepsilon_{\mathrm{f}}=1,91 \% \mathrm{o}, \\
& \beta_{\mathrm{w}}=400 \mathrm{~kg} / \mathrm{cm}^{2}, \quad \beta=320 \mathrm{~kg} / \mathrm{cm}^{2}, \quad \tau_{\mathrm{v}}^{*}=60 \mathrm{~kg} / \mathrm{cm}^{2}, \\
& d / \mathrm{h}=0,5, \quad b_{\mathrm{o}} / \mathrm{b}=1,0, \quad \sigma_{\mathrm{bz}} / \tau_{\mathrm{vr}}=0,5 .
\end{aligned}
$$

$\mathrm{Da} \mu_{\mathrm{Gr}}^{*}$ kleiner als $\mu_{\mathrm{Gr}}$ aus ( 112 ) beziehungsweise ( 117) wird, ist $\mathrm{z} / \mathrm{s}>1,0$, 
sodass sich hier eine Auswertung und Darstellung von $\mu_{\mathrm{Gr}}$ nach ( 116 ) erübrigt.

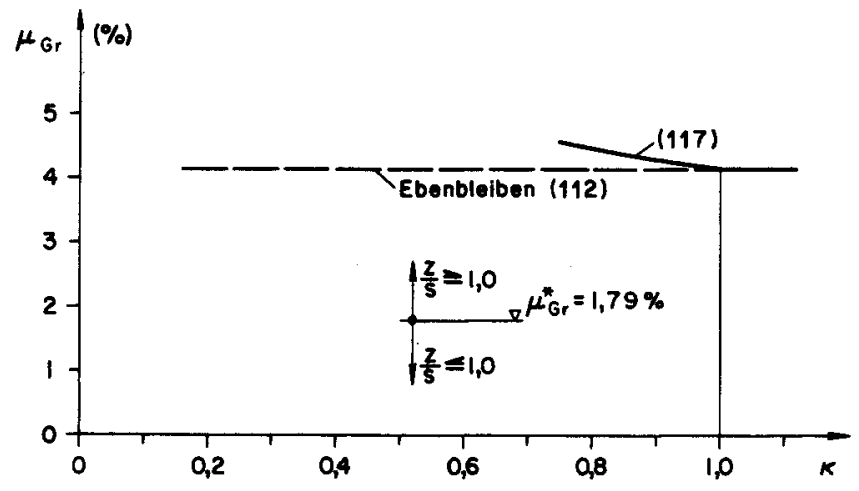

\section{Bild 30}

Armierungsgehalt zur Charakterisierung der Sprödbruchgefahr in Abhängigkeit vom Verbundkoeffizienten (Beispiel)

Die Ausdrücke ( 112 ) und ( 117 ) sind bis auf $\kappa$ im Nenner identisch. Es soll deshalb noch abgeschätzt werden, welches der Variationsbereich von $\kappa$ für $\varepsilon_{\text {emax }}=\varepsilon_{\mathrm{f}}$ und dessen Einfluss auf $\mu_{\mathrm{Gr}}$ ist. Mit dem elastisch-plastischen Spannungs-Dehnungs-Diagramm nach Bild 2 ergibt sich:

$$
\kappa=1-\frac{\Delta G_{e t o t}}{2 \cdot \sigma_{f}}
$$

Daraus wird mit Hilfe der Gleichungen ( 26) und (63a) für $\mu=\mu_{\mathrm{Gr}}$ :

$$
\varepsilon_{\text {emax }}=\varepsilon_{f}: \quad \kappa=1-0,2 \cdot \frac{\tau_{v}^{*}}{\sigma_{f}} \cdot \frac{\sigma_{b z}}{\tau_{v r}} \cdot \frac{b_{o}}{b} \cdot \frac{1}{\mu_{G r}}
$$


Für übliche Verhältnisse liegt bei Stahlbetonbalken $\mu_{\mathrm{Gr}}$ nach (112) in der Grössenordnung von 2 bis $5 \%$. Setzt man im übrigen die weiter oben aufgeführten Zahlenwerte ein, so variiert $\kappa$ von 0,925 bis 0,97 . Ganz allgemein kann festgestellt werden, dass besonders bei höheren Armierungsgehalten der Verbundkoeffizient $\kappa$ nur verhältnismässig wenig kleiner als 1,0 ist, solange die Längsarmierung noch keine plastischen Deformationen erlitten hat. Daher ist der Einfluss von $\kappa$ auf $\mu_{\mathrm{Gr}}$ nicht von Bedeutung, was aus Bild 30 deutlich hervorgeht. In dem für $\kappa$ in Frage kommenden Bereich verläuft die Funktion ( 117 ) nur wenig iber der Geraden nach ( 112 ). Letztere befindet sich, was die Sprödbruchgefahr betrifft, ausserdem auf der sicheren Seite.

Fasst man die Ergebnisse der vorangehenden Vergleiche mit der üblichen Betrachtungsweise kurz zusammen, so kann die folgende allgemeine Erkenntnis formuliert werden:

Solange die Zugarmierung keine plastischen Deformantionen erleidet, ergeben sich in einem vorwiegend auf Biegung beanspruchten Balkenabschnitt - was das Verhalten der Zugarmierung betrifft - bei einer Berechnung auf der Grundlage des Ebenbleibens der Querschnitte nur verhältnismässig geringe Fehler gegenüber dem tatsächlichen Verhalten. Sobald jedoch die Zugarmierung plastische Deformationen aufweist, sowie was das Verhalten des Betons in der Biegedruckzone betrifft, kann die Hypothese vom Ebenbleiben der Querschnitte zu schwerwiegenden Fehlschlüssen führen. 


\subsection{Allgemeine Beurteilung}

\subsection{Verhalten des Biegeriss-Gelenkes}

Wie die Darlegungen dieses Kapitels zeigen, hängt die Deformationsfahigkeit eines Biegeriss-Gelenkes in hohem Masse von verschiedenen Parametern ab. Im Vergleich zu dem für eine Umlagerung der innern Kräfte erforderlichen Winkel (siehe 4.) kann der kritische Gelenkwinkel oft ausserordentlich gross, bisweilen aber auch ungentigend klein sein. Besonders ungünstig wirkt sich in einem Biegeriss-Gelenk eine verhăltnismăssig grosse Schubbeanspruchung aus. Ist diese derart, dass gerade noch keine Biegeschubrisse entstehen (vergleiche 3.11), es sich also tatsächlich um ein Biegeriss-Gelenk handelt, so können sich die plastischen Deformationen auf einen kleinen Bereich, bei grösserem Rissabstand eventuell sogar auf den Bereich eines einzigen Blegerisses konzentrieren. Sofern in diesem Fall Versagen des Betons am Druckrand massgebend ist, so tritt es bei einem ziemlich kleinen Gelenkwinkel ein. Unter Umständen kann jedoch auch die Zugarmierung versagen, sofern sie bei guten Verbundeigenschaften kleine Stabdurchmesser und/oder mässige bis schlechte Dehnungs- und VerfestigungsEigenschaften aufweist. Bei Biegeriss-Gelenken ist es deshalb oft nötig, sich über die Grösse des kritischen Gelenkwinkels Rechenschaft zu geben.

Ist die Schubbeanspruchung jedoch gering, beziehungsweise stellt man zu Beginn der Untersuchung fest, dass in mehreren Rissen (mindestens drei) plastische Deformationen auftreten, so erübrigt sich in manchen Fallen eine genauere Berechmung des kritischen Gelenkwinkels.

\subsection{Momenten-Rotations-Charakteristik}

Die vorangehenden Untersuchungen zeigen ferner eindrücklich, dass in BiegerissGelenken kein einfacher Zusammenhang zwischen den Grössen Moment und Rotationswinkel, und schon gar nicht zwischen Moment und "Krümmung" besteht, wie ihn manche Autoren immer wieder voraussetzen. Bei einem bestimmten Mo- 
ment hängt die Grösse des Gelenkwinkels von denselben zahlreichen Parametern $\mathrm{ab}$, welche die Deformationsfăhigkeit entscheidend beeinflussen. Auch durch Versuche erarbeitete Momenten-Rotationskurven gelten somit nur für die betreffenden Parameter-Konstellationen. Schon die Anwendung solcher Kurven auf scheinbar nur leicht veränderte Verhältnisse kann $\mathrm{zu}$ beträchtlichen Fehlschlüssen

führen. Der Begriff der Momenten-Rotations-Charakteristik ist deshalb kaum ein taugliches Mittel, um das Verformungsverhalten plastischer Stahlbeton-Gelenke zu erfassen. Vielmehr muss durch eine verfeinerte Betrachtungsweise anhand der hier entwickelten Grundlagen versucht werden, die Einflüsse der einzelnen Parameter zu bericksichtigen. 


\section{DAS SCHUBRISS - GELENK}

\subsection{Modell}

\subsection{Definition}

Bild 31 zeigt schematisch ein Schubriss-Gelenk. Solche Gelenke treten vor allem dort auf, wo verhăltnismässig konzentrierte Kräfte senkrecht zur Stabachse auf das Tragwerk wirken. Dies ist bei Einzellasten, sowie vor allem bei Zwischenstiutzen der Fall, weshalb in Bild 31 die Zugseite oben dargestellt ist.

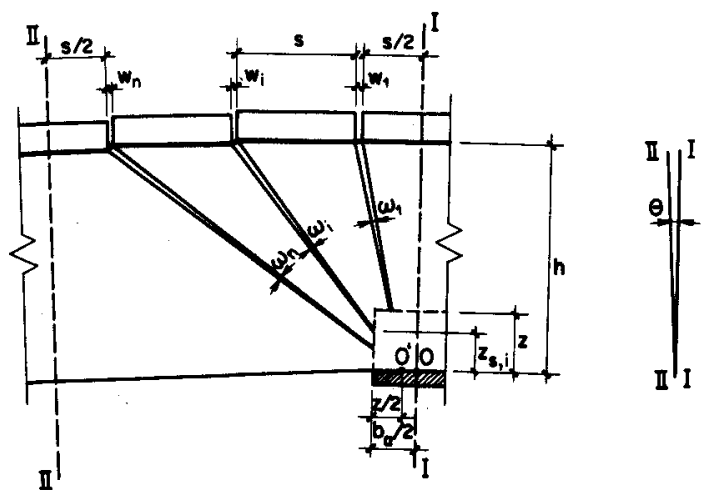

Bild 31

Modell des SchubrissGelenkes

In einem Schubriss-Gelenk ist ausser dem Biegemoment noch eine erhebliche Schubbeanspruchung vorhanden. Diese hat einen wesentlichen Einfluss auf die Ausbildung des Gelenkes, indem sogenannte Biegeschubrisse entstehen. Solche entwickeln sich meist aus Biegeanrissen und verlaufen zuerst ungefähr senkrecht zur Stabaxe bis etwa zur Zugarmierung. Nachher pflanzen sie sich unter dem Einfluss der Schubbeanspruchung gegen die Stelle der Krafteinleitung hin fort. Die Form der Biegeschubrisse ist meist mehr oder weniger konvex zur Zugarmierung. Die Risse verlaufen umso gestreckter und geradliniger, je grösser die Schubbeanspruchung ist. Sie dringen umso năher zum Druckrand hin vor, je 
stărker sie geneigt sind. Typische Schubriss-Gelenke sind in den Arbeiten [6], [7] und [8] abgebildet.

Aus Biegeanrissen entstehen Biegeschubrisse, sofern die am jeweiligen Ende des Risses auftretenden Spannungen im noch ungerissenen Bereich eine gewisse Neigung zur Stabaxe aufweisen. Ein Versuch zur Berechnung von Grösse und Richtung der Spannungen am Ende von Biegeschubrissen und zur Vorhersage des Risseverlaufes ist kürzlich unternommen worden [21]. Das an und für sich interessante und geschickte Verfahren ist jedoch an verschiedene Voraussetzungen gebunden und ziemlich aufwendig und kompliziert, sodass es hier kaum angewendet werden kann. Für die Abgrenzung zwischen Biegeriss- und Schubriss-Gelenk müssen wir uns daher mit einer Năherungsregel begnügen.

Als Mass für die Schubbeanspruchung definieren wir in Anlehnung an die Arbeit [9] die nominelle Schubspannung $\tau$ wie folgt:

$$
\tau=\frac{Q}{b_{0} h}
$$

$Q$ bedeutet die Querkraft, $b_{o}$ die Stegbreite und h die statische Höhe. Die nominelle Schubspannung charakterisiert die Beanspruchung eines Balkensteges durch schiefe Zug- und Druckkräfte. Aus Versuchsresultaten, insbesondere aus [7] und [8], kann geschlossen werden, dass mit dem Auftreten von Biegeschubrissen beziehungsweise Schubriss-Gelenken uiberall dort gerechnet werden kann, wo die folgende Bedingung erfüllt ist:

$$
T\left(\varepsilon_{f}\right)=\tau_{1}
$$

$\tau\left(\varepsilon_{\mathrm{f}}\right)$ bedeutet die nominelle Schubspannung im plastischen Gelenk beim Erreichen der nominellen Fliessdehnung in der Zugarmierung (vergleiche Bild 2), das heisst also etwa beim Beginn der plastischen Rotation im Gelenk. $\tau_{1}$ ist der folgenden Tabelle zu entnehmen: 


\begin{tabular}{|cc|c|c|c|c|}
\hline$\beta_{\mathrm{w}}$ & $\left(\mathrm{kg} / \mathrm{cm}^{2}\right)$ & 200 & 300 & 400 & $\geq 500$ \\
\hline$\tau_{1}\left(\mathrm{~kg} / \mathrm{cm}^{2}\right)$ & 8 & 10 & 12 & 14 \\
\hline
\end{tabular}

$\beta_{w}$ bedeutet die Würfelfestigkeit des Betons. $\tau_{1}$ entspricht der sogenannten unteren Schubspannungsgrenze nach [9] , bei deren Ueberschreitung eine Schubarmierung anzuordnen ist. Der Rotationsbeginn wird deshalb als massgebend betrachtet, weil bis zu seinem Erreichen sich die meisten Risse des Gelenkes ganz oder weitgehend ausgebildet haben. Auch ist die Grenze zwischen Schubriss-Gelenk und Biegeriss-Gelenk eher zu ungunsten des Schubriss-Gelenkes anzusetzen. Letzteres weist, wie bereits in [6] gezeigt wurde, meistens einen grösseren kritischen Gelenkwinkel auf als das Biegeriss-Gelenk. Im Zweifelsfalle liegt man daher mit der Annahme von Biegerissen respektive eines Biegeriss-Gelenkes bezïglich Rotationsfähigkeit im allgemeinen auf der sicheren Seite.

Im Modell des Schubriss-Gelenkes, Bild 31, verlaufen die Biegeschubrisse von der Zugarmierung weg geradlinig auf den Punkt $O^{\prime}$ hin, welcher vom Rand der Krafteinleitung den Abstand $z / 2$ hat. Sie enden an dem Rechteck, welches mit den Strecken $z$ und $b_{a}$ gebildet werden kann. Dieses ist in Bild 31 gestrichelt eingetragen. $\mathrm{z}$ bedeutet die Strecke vom Ende eines Biegerisses bis zur Druckkante, wie sie in Gleichung ( 5 ) beziehungsweise ( 6 ) definiert worden ist. $b_{a}$ bezeichnet die Breite, iber welche die Einzelkraft eingeleitet wird. Im folgenden wird angenommen, dass

$$
b_{a}=z
$$

Wie beim Biegeriss-Gelenk wird vorausgesetzt, dass innerhalb des Gelenkes die Art der Zugarmierung (Querschnitt, Stabdurchmesser, Spanmungs-DehnungsDiagramm, Verbundeigenschaften) sowie der Balkenquerschnitt sich nicht verändern. Ferner gelten die Ausführungen in diesem Kapitel ausdrücklich nur für Schubriss-Gelenke mit einer Schubarmierung, welche aus zur Stabaxe senkrecht stehenden Bügeln besteht. Der Fall einer schrägen oder gemischten Schubarmierung wird hier nicht behandelt. 
Das plastische Gelenk soll wiederum diejenigen Risse umfassen, in denen plastische Deformationen der Zugarmierung auftreten:

$$
\varepsilon_{\mathrm{eR}}=\varepsilon_{\mathrm{f}}
$$

Biegeschubrisse können natürlich nur dort entstehen, wo bereits Biegeanrisse sind. Es gelten also auch hier die Gleichungen ( 3 ) und ( 4 ).

Vernachlăssigt man die im allgemeinen geringe Distanz zwischen den Punkten $O$ und $O^{\prime}$ nach Bild 31, und setzt man einen geradlinigen Verlauf der Momentenfläche im Bereich des Gelenkes voraus, so gilt für die Neigung des flachsten Biegeschubrisses $n$, für welchen ( 4 ) erfüllt ist, die folgende Bedingung:

$$
\operatorname{tg} \delta_{n}=\frac{M_{I}-M_{R}}{Q \cdot h}
$$

$\delta_{n}$ bedeutet den Winkel, den der Riss im Modell gegenüber einer Senkrechten zur Balkenachse aufweist (vergleiche Bild 32). $M_{I}$ ist das Biegemoment im Schnitt I - I, ohne Berücksichtigung der Wirkung von Pressungen im Bereich $b_{a} / 2$.

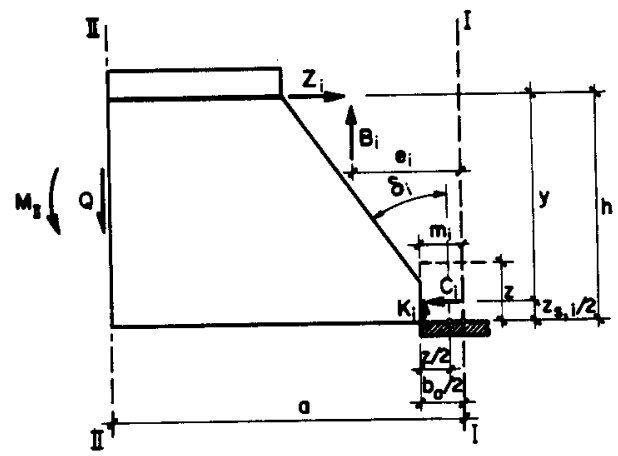

Bild 32

Innere Kräfte im Schubriss-Gelenk 
Im Gegensatz zum Biegeriss-Gelenk genügen im Schubriss-Gelenk die Bedingungen ( 2 ) und ( 3 ) noch nicht, um die zum Gelenk gehörigen Risse zu bestimmen. Denn im Rahmen von ( 2 ) beziehungsweise ( 3 ) können nicht beliebig viele, das heisst beliebig geneigte, Biegeschubrisse zum Gelenk gezählt werden. Aus den Versuchen [7] und [8] geht hervor, dass im allgemeinen für die Neigung des flachsten Gelenkrisses $n$ gilt:

$$
\operatorname{tg} \delta_{n} \leq 1,3
$$

Es fallen somit nur Anrisse, die weniger oder höchstens 1,3 h vom Schnitt I - I, beziehungsweise von dessen Parallelen durch $\mathrm{O}^{\prime}$, entfernt liegen, als Biegeschubrisse des Gelenkes in Betracht. Aus Anrissen, die in grösserer Entfernung als $1,3 \mathrm{~h}$ von $\mathrm{O}^{\prime}$ entstehen, entwickeln sich oft Biegeschubrisse, welche nicht flach bis zum Bereich der Krafteinleitung, sondern etwa parallel zum benachbarten Biegeschubriss $n$ verlaufen und in meist verhältnismässig grossem Abstand von der Druckkante enden. Auch können sich eigentliche Schubrisse, die im Steg im Bereich der Balkenachse entstehen, später bis zum Zugrand fortpflanzen und dann die Form vom Biegeschubrissen haben. Diese beiden Rissarten können jedoch nicht in das Schubriss-Gelenk einbezogen werden.

Der als konstant angenommene Abstand $\mathbf{s}$ der Biegeschubrisse am Zugrand wird weitgehend durch dieselben Parameter beeinflusst, wie derjenige von Biegerissen in Biegebereichen. Zur Bestimmung von s können daher die im Abschnitt 2.3 angegebenen Regeln verwendet werden.

Im Bereiche der Krafteinleitung (Auflager) sind die Verhältnisse ähnlich wie in einem Biegeriss-Gelenk. Der Hebelarm der innern Kräfte y kann daher ebenfalls mit Gleichung ( 7 ) bestimmt werden.

\subsection{Gelenkwinkel}

Der Gelenkwinkel $\Theta$ in einem Schubriss-Gelenk wird als gegenseitige Verdrehung der Betonquerschnitte I - I und II - II gemäss Bild 31 definiert. Der Querschnitt I - I geht durch die Mitte der Strecke $b_{a}$, längs welcher die Einzelkraft einge- 
leitet wird. Es wird ferner angenommen, dass der erste Biegeschubriss vom Querschnitt I - I den Abstand s/2 habe. Der Querschnitt II - II soll vom letzten Riss ebenfalls den Abstand $\mathrm{s} / 2$ aufweisen.

Werden die parallel zur Zugarmierung zu messende Rissbreite wiederum mit w und die Strecke vom Ende eines Biegeschubrisses bis zur Druckkante mit $z_{s}$ bezeichnet, so gilt für den einzelnen Risswinkel:

$$
\omega_{i}=\frac{w_{i}}{h-z_{s, i}}
$$

Setzt man vereinfachend für alle Risse

$$
\mathbf{h}-\mathrm{z}_{\mathbf{s}, \mathbf{i}} \cong \mathbf{h}-\mathbf{z}
$$

so ergibt sich, wenn die Betondehnungen zwischen den Rissen vernachlässigt werden:

$$
\omega_{i}=\frac{w_{i}}{h-z} \quad(10) \quad \theta=\frac{\sum_{1}^{n} w_{i}}{h-z}=\sum_{1}^{n} \omega_{i}
$$

Dies sind dieselben Beziehungen, wie sie für das Biegeriss-Gelenk gelten.

\subsection{Innere Kräfte}

In diesem Abschnitt soll der Zusammenhang zwischen den in einem SchubrissGelenk wirkenden inneren Kräften untersucht werden.

Ausgehend von Bild 31 ist in Bild 32 der links vom i-ten Biegeschubriss bis zum Schnitt II - II vorhandene Gelenk - beziehungsweise Balkenteil dargestellt. Die im Bereich der Krafteinleitung (Auflager) gewählte Begrenzung ist ebenfalls ersichtlich. Sie soll jeweils geradlinig vom Ende des betreffenden Biegeschubrisses bis zum Rand der Krafteinleitung verlaufen. Auch wurden in Bild 32 die 
auf diesen Teil wirkenden Kräfte beziehungsweise Momente eingetragen. Dabei wird vorausgesetzt, dass zwischen den Schnitten I - I und II - II keine bussere Belastung wirkt.

Im Schnitt II - II greifen das Biegemoment $M_{I I}$ und die Querkraft $Q$ an. $Z_{i}$ bedeutet die Kraft in der Zugarmierung. $B_{i}$ ist die Resultierende aller Bügelkräfte links vom Rand der Krafteinleitung in dem gegen den Schnitt I - I um den Winkel $\delta_{i}$ geneigten Biegeschubriss. $C_{i}$ und $K_{i}$ sind die in der Biegedruckzone parallel beziehungsweise senkrecht zur Balkenaxe angreifenden Kräfte. Die geometrischen Grössen zur Festlegung sämtlicher Wirkungslinien sind in Bild 32 ebenfalls eingetragen.

\subsection{Kräfte in der Zugarmierung}

Das Momentengleichgewicht bezüglich dem Punkt $O$ lautet:

$$
M_{I I}+Q_{a}-Z_{i} h-B_{i} e_{i}-K_{i} m_{i}+C_{i} \cdot \frac{z_{, i}}{2}=0
$$

Die Summen der horizontalen beziehungsweise vertikalen Kräfte betragen:

$$
\begin{aligned}
& C_{i}-Z_{i}=0 \\
& Q-B_{i}-K_{i}=0
\end{aligned}
$$

Da zwischen den Schnitten I - I und II - II keine äussere Belastung wirkt, kann gesetzt werden:

$$
\mathbf{M}_{\mathbf{I}}=\mathbf{M}_{\mathbf{I I}}+\mathbf{Q} \cdot \mathbf{a}
$$

Mit dem Hebelarm der innern Krăfte

$$
y \cong h-\frac{z_{s, i}}{2}
$$


respektive in Anlehnung an Gleichung ( 127 ),

$$
y \cong h-\frac{z}{2}
$$

wird die Kraft in der Zugarmierung:

$$
z_{i}=\frac{M_{I}}{y}-B_{i} \cdot \frac{e_{i}}{y}-K_{i} \cdot \frac{m_{i}}{y}
$$

Das erste Glied bedeutet die Zugkraft in einem Biegeriss-Gelenk beziehungsweise in einem nur durch ein Biegemoment beanspruchten Bereich. Der zweite Summand charakterisiert den Abbau der Zugkraft durch die Buigelkräfte. Dieser ist umso stärker, je grösser die Resultierende $B_{i}$ und deren Hebelarm sind. Das letzte Glied entspricht gewissermassen der Ausrundung der Momentenfläche durch die Krafteinleitung.

Führt man noch die Querkraft $Q$ sowie

$$
\mathbf{y}=\eta \cdot \mathrm{h}
$$

ein, so wird aus ( 133$)$ mit ( 130$)$ :

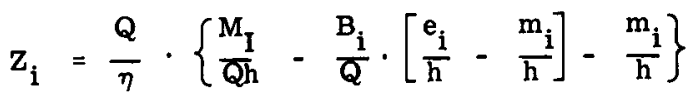

In einem Schubriss-Gelenk sollte stets eine möglichst fein verteilte Schubarmierung mit einem Bügelabstand $t \leq h / 4$ angeordnet sein. Die Bügelkräfte werden daher näherungsweise als gleichmässig verteilt über die Risslänge neben der Krafteinleitung, das heisst über die Strecke $h \cdot \operatorname{tg} \delta_{i}-z / 2$ angenommen. Bedeutet $B_{45}$ die Summe der Bügelkräfte in einem $45^{\circ}$-Riss, so ergibt sich folgender Zusammenhang: 
$\operatorname{tg} \delta_{i} \geq \frac{z}{2 h}: \quad B_{i}=B_{45} \cdot\left[\frac{2 \operatorname{tg} \delta_{i}-z / h}{2-z / h}\right]$

$\operatorname{tg} \delta_{i}=\frac{z}{2 h}: \quad B_{i}=0$

Auf derselben Grundlage erhält man das Hebelarmverhältnis $e_{i} / h$ von $B_{i}$ für

$\operatorname{tg} \delta_{i}=\frac{z}{2 h}: \quad \frac{e_{i}}{h}=\frac{\operatorname{tg} \delta_{i}}{2}-\frac{1}{4} \cdot \frac{z}{h}+\frac{1}{2} \cdot \frac{b_{a}}{h}$

Ferner beschreibt die Wirkungslinie von $\mathrm{K}_{\mathrm{i}}$ :

$\operatorname{tg} \delta_{i} \geq 0,5: \quad \frac{m_{i}}{h}=\frac{1}{2} \cdot \frac{b}{h}$

$\operatorname{tg} \delta_{i}=0,5: \quad \frac{m_{i}}{h}=\frac{\operatorname{tg} \delta}{2} \cdot \frac{z}{h}-\frac{1}{4} \cdot \frac{z}{h}+\frac{1}{2} \cdot \frac{b_{a}}{h}$

$\mathrm{Da}$ jedoch ( $138 \mathrm{~b}$ ) nur für einen kleinen und verhältnismässig unbedeutenden $\mathrm{Be}-$ reich von $\delta_{i}$ gilt und ausserdem der begangene Fehler gering ist, wird im folgenden der Gültigkeitsbereich von (138a) mit $\operatorname{tg} \delta_{i}=z / 2 h$ angenommen.

Setzt man die Ausdrücke (136a), ( 137 ) und (138a) in (135) ein, so ergibt sich für $\operatorname{tg} \delta_{i}=z / 2 h:$

$$
\mathrm{Z}_{\mathrm{i}}=\frac{\mathrm{Q}}{\eta} \cdot\left[\frac{\mathrm{M}_{\mathrm{I}}}{\mathrm{Qh}}-\frac{\mathrm{B}_{45}}{\mathrm{Q}} \cdot \frac{\left(\operatorname{tg} \delta_{\mathrm{i}}-\mathrm{z} / 2 \mathrm{~h}\right)^{2}}{2(1-\mathrm{z} / 2 \mathrm{~h})}-\frac{\mathrm{b}_{\mathrm{a}}}{2 \mathrm{~h}}\right]
$$

Von grosser Bedeutung ist auch die Differenz der Zugkräfte in zwei benachbarten Biegeschubrissen:

$$
\Delta z_{i, i+1}=z_{i}-z_{i+1}
$$


$\Delta Z_{i, i+1}=-\frac{Q}{\eta} \cdot \frac{B_{45}}{Q} \cdot\left[\frac{\left(\operatorname{tg} \delta_{i}-z / 2 h\right)^{2}-\left(\operatorname{tg} \delta_{i+1}-z / 2 h\right)^{2}}{2(1-z / 2 h)}\right]$

Nach ( 139 ) ergibt sich somit im wesentlichen die Zugkraft $Z_{i}$ aus der Multiplikation der Querkraft mit einem Faktor, welcher aus der Differenz zwischen dem Momenten-Schub-Verhältnis und einem Glied besteht, das die Einflüsse des Quotienten $B_{45} / Q$ und geometrischer Grössen erfasst.

\subsection{Kräfte in der Bügelarmierung und der Biegedruckzone}

( 139 ) und ( 140 ) zeigen, dass von wesentlicher Bedeutung für die Veränderung der Zugkraft in einem Schubriss-Gelenk das Verhältnis $B_{45} / Q$ ist, das heisst, derjenige Querkraftsanteil, welcher von den Bügeln in einem $45^{\circ}$-Riss übernommen wird, im Vergleich zur gesamten Querkraft. Wird der im $45^{\circ}$-Riss curch die Biegedruckzone ubertragene Anteil an der Querkraft mit $\mathrm{K}_{45}$ bezeichnet, so gilt in Analogie zu ( 130 ):

$$
B_{45}=Q-K_{45}
$$

Die Aufteilung der Querkraft auf Schubarmierung und Biegedruckzone ist grundsätzlich eine Frage der geometrischen Verträglichkeit der inneren Verformungen (Kompatibilität). Es ist sehr wohl möglich, dass in einem Stahlbetonbalken trotz dem Vorhandensein von Bügeln ein verhältnismässig grosser Teil der Querkraft durch die Biegedruckzone übertragen wird. Dies kann zum Beispiel der Fall sein, wenn die Längsarmierung nicht über die Proportionalitätsgrenze hinaus beansprucht ist und sich demzufolge die Biegeschubrisse noch nicht voll ausgebildet haben. In diesem Bereich ist eine rechnerische Erfassung von $B_{45}$ beziehungsweise $\mathrm{K}_{45}$ praktisch unmöglich. Hingegen stellt sich nach dem Auftreten von plastischen Verformungen in der Längsarmierung eine von verschiedenen Parametern abhängige Grösse $K_{45}$ ein. In der Arbeit [9] wurde auf Grund von Versuchsresultaten ein "Schubwiderstand der Biegedruckzone" am Ende eines $45^{\circ}$-Biege- 
schubrisses wie folgt definiert:

$$
Q_{C}=\tau_{1} \cdot b_{0} \cdot h
$$

Die Grösse von $\tau_{1}$ wurde bereits im Abschnitt 3.11 in Abhängigkeit von der Würfeldruckfestigkeit des Betons angegeben. $b_{0}$ ist die Stegbreite und $h$ die statisch wirksame Höhe. $Q_{C}$ wurde in [9] ausdrücklich als Widerstandsgrösse bezeichnet und dient dort unter anderem zur Bemessung der Schubarmierung. Ein allfälliger Schubbruch soll nicht vor dem Biegebruch eintreten, beziehungsweise nicht bevor die Längsarmierung bis etwa zur Streckgrenze gedehnt ist. Wie die Versuche [7] und insbesondere [8] zeigten, bleibt jedoch die von der Biegedruckzone übertragene Querkraft auch bei fortschreitender Rotation, das heisst bei grösseren Verformungen der Längsarmierung, über weite Bereiche erhalten. Es kann somit für unsere Zwecke gesetzt werden:

$$
K_{45} \cong Q_{C}
$$

Damit wird aus ( 141$)$ :

$$
\mathbf{B}_{45}=\mathbf{Q}-\mathbf{Q}_{\mathbf{C}}
$$

\subsection{Schiefe Druckkräfte}

Im folgenden werden noch die schiefen Druckkräfte angegeben, welche in einem Schubriss-Gelenk auftreten. In Bild 33 ist ein Bereich zwischen zwei Biegeschubrissen (Druckstrebe) mit den daran angreifenden Kräften dargestellt. Nimmt man an, dass die Bügelkrăfte $B_{i}$ und $B_{i+1}$ über die jeweiligen Risslängen neben dem Rand der Krafteinleitung gleichmässig verteilt seien, so wird mit ( 136a ) deren Resultierende: 


$$
\Delta B_{i, i+1}=B_{i}-B_{i+1}=B_{45} \cdot\left[\frac{\operatorname{tg} \delta_{i}-\operatorname{tg} \delta_{i+1}}{1-z / 2 h}\right]
$$

Unter der Voraussetzung, dass die Einspannung der Druckstrebe am untern Ende vernachlässigbar gering sei, entspricht der Winkel $\delta_{i, i+1}$ (Wirkungslinie der Druckkraft $D_{i, i+1}$ ) der Winkelhalbierenden der beiden Risswinkel:

$$
\delta_{i, i+1}=\frac{\delta_{i}+\delta_{i+1}}{2}
$$

Da die Differenz der Bügelkräfte, beziehungsweise deren Resultierende $\Delta B_{i, i+1}$ nach ( 145 ), in der Mitte zwischen den Biegeanrissen angreift, wird der Winkel $\delta_{i, i+1}$ - wie in Bild 33 gezeichnet - jedoch zweckmässigerweise wie folgt angenommen:

$$
\delta_{i, i+1} \cong \operatorname{arctg}\left[\frac{\operatorname{tg} \delta_{i}+\operatorname{tg} \delta_{i+1}}{2}\right]
$$

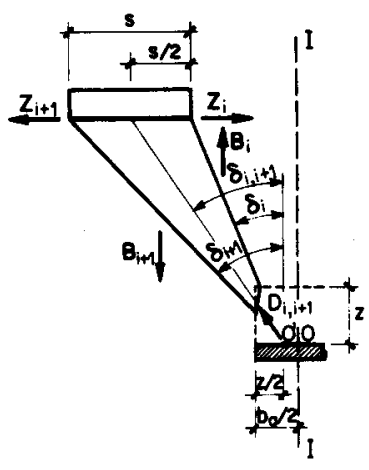

$\underline{\text { Bild } 33}$

Bereich zwischen zwei Biegeschubrissen mit angreifenden Kräften 
Die Differenz zwischen ( $146 a)$ und (146b) ist unbedeutend. Die Annahme von (146b) vernachlässigt lediglich eine geringe Exzentrizität von $D_{i, i+1}$ beim $\mathrm{Zu}$ sammentreffen mit $\Delta B_{i, i+1}$ auf der Höhe der Zugarmierung. Damit ergibt sich die Druckkraft $D_{i, i+1}$ aus dem Gleichgewicht der Kräfte zu

$$
D_{i, i+1} \cong \frac{\Delta z_{i, i+1}}{\sin 8_{i, i+1}},
$$

oder auch

$$
D_{i, i+1} \cong-\frac{\Delta B_{i, i+1}}{\cos \delta_{i, i+1}}
$$

In ( 147a) kann $\Delta \mathrm{Z}_{\mathbf{i}, \mathbf{i}+1}$ nach ( 140 ) verwendet werden.

Aus ( $147 \mathrm{a}$ ) und ( $147 \mathrm{~b}$ ) wird ferner:

$$
\Delta Z_{i, i+1}=-\Delta B_{i, i+1} \cdot \operatorname{tg} \delta_{i, i+1}
$$

\subsection{Kritischer Gelenkwinkel}

Der "kritische Gelenkwinkel" in einem Schubriss-Gelenk ist dann vorhanden; wenn einer der folgenden wichtigsten "kritischen Zustände" erreicht ist:

- Versagen der Zugarmierung

- Versagen des Betons

- Versagen der Bügelarmierung

- Versagen des Verbundes der Zugarmierung

Der kritische Gelenkwinkel wird - analog wie im Biegeriss-Gelenk - bei n Biegeschubrissen des Gelenkes: 


$$
\theta_{k r}=\frac{-111-}{h-z}=\operatorname{krit} \sum_{1}^{n} w_{i} w_{i}
$$

Vor der Behandlung der verschiedenen Arten des Versagens werden noch allgemein die Verformungen der Zugzone betrachtet.

\subsection{Verformungen der Zugzone zwischen zwei Biegeschubrissen}

Wie beim Biegeriss-Gelenk werden beim Schubriss-Gelenk die Verformungen des Betons in der Zugzone gegenüber denjenigen der Zugarmierung vernachlässigt. In Bild 34 sind für die Zugarmierung die Dehnungen und die Verbundspannungen im Bereich zwischen zwei Biegeschubrissen dargestellt. Der Verlauf dieser Grössen sowie die allgemeinen Zusammenhänge sind grundsätzlich dieselben wie im $\mathrm{Be}-$ reich zwischen zwei Biegerissen in einem Biegeriss-Gelenk mit variablem Biegemoment (vergleiche Bild 17). Es gelten somit die zu Beginn des Abschnittes 2.41 b) dargestellten Beziehungen. Die unterschiedlichen Zugkräfte in zwei benachbarten Rissequerschnitten bewirken eine ungleichmässige Verteilung der Verbundspannungen. Deren Nullstelle zwischen den Rissen entspricht der Stelle, wo die Relativverschiebung zwischen dem Beton und der Zugarmierung verschwindet. Die grössten Verschiebungen entstehen in den Rissequerschnitten, wo sie zur jeweiligen Rissbreite beitragen.

Für eine konkrete Berechnung ist es jedoch in vielen Fällen - ähnlich wie im Biegeriss-Gelenk - nicht nötig, den Einfluss der Querkraft auf den Verlauf der Stahldehnungen zwischen den Rissen zu berücksichtigen. Es können vielmehr die im Abschnitt 2.21 für einen Bereich zwischen zwei Biegerissen, der ausschliesslich durch ein Biegemoment beansprucht wird, hergeleiteten Beziehungen verwendet werden. Diese nehmen die Lage des Verschiebungsnullpunktes in der Mitte zwischen den zwei Rissen an. Sie können ferner hier - gleich wie im Abschnitt 2.41 a) bei der Berechnung eines auch durch Querkräfte beanspruchte Biegerissgelenkes - angewendet werden unter der Voraussetzung, dass die Stahldehnungen jeweils symmetrisch zu den Rissequerschnitten verlaufen. Diese Vereinfachun- 


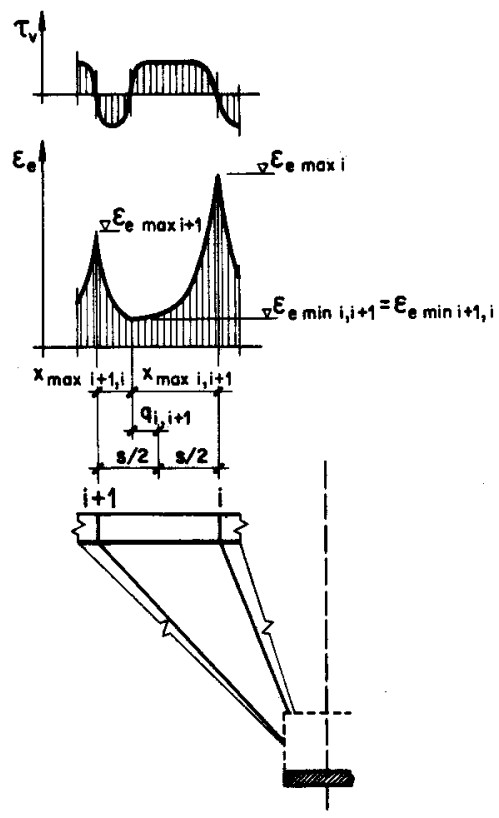

Bild 34

Genereller Verlauf der Stahldehnungen und Verbundspannungen der Zugarmierung im Bereich zwischen zwei Biegeschubrissen

gen rechtfertigen sich umsomehr, als infolge der Rotation beziehungsweise durch Dübelwirkung unmittelbar beidseits der Rissequerschnitte Querpressungen auf die Zugarmierung wirken. Die entsprechende Reibung kann das Verbundgesetz verändern, beziehungsweise die dortiger. Verbundspannungen erhöhen. Da dieser Effekt theoretisch nicht erfasst wird, ist eine allzu genaue Berechnung ohnehin nicht angebracht.

Die Ermittlung der Verformungen im Bereich zwischen zwei Biegeschubrissen eines Schubriss-Gelenkes lässt sich somit analog wie diejenige zwischen zwei benachbarten Biegerissen eines Biegeriss-Gelenkes durchführen. Mehr Schwierig- 
keiten als im Biegeriss-Gelenk bereitet im Schubriss-Gelenk in einzelnen Fällen die Berechnung der Verformungen des ganzen Gelenkes. Dieses Problem wird in den folgenden Abschnitten jeweils zusammen mit den verschiedenen Arten des Versagens behandelt.

\subsection{Versagen der Zugarmierung}

Bei bestimmten Verhältnissen kann, wie im Biegeriss-Gelenk, auch im Schubriss-Gelenk das Versagen beziehungsweise der Beginn der Einschnürung der Zugarmierung zu einer Reduktion des übertragbaren Biegemomentes und zum Bruch führen. Unter den früher getroffenen Voraussetzungen wird dies der Fall sein im Bereich des maximalen Biegemomentes, das heisst, im steilsten Biegeschubriss, der meist eher ein Biegeriss ist. Für die Dehnung der Zugarmierung beim Beginn der Einschnürung, die kritische Rissweite beziehungsweise den kritischen Risswinkel, und so weiter, gelten die Ausführungen und Formeln in Abschnitt 2.22 ohne Aenderung.

Desgleichen kann die Berechnung von $\Theta_{\mathrm{kr}}$ ähnlich wie beim Biegeriss-Gelenk durchgeführt werden (vergleiche 2.41 a)). Das Vorgehen ist folgendes:

1. Im kritischen Riss gilt nach ( 36 ) beziehungsweise ( 40$) \varepsilon_{\text {e } \max }=\varepsilon_{\mathrm{er}} \cong$ $\lambda_{\mathrm{Gl}}$ und $\sigma_{\mathrm{e} \max }=\beta_{\mathrm{z}}$.

2. In den übrigen Rissen des Gelenkes kann die maximale Stahlspannung $\sigma_{\text {e } \max i}$ aus ( 139 ) beziehungsweise mit Hilfe von (140) ermittelt werden.

3. Damit sind aus dem Spannungs-Dehnungs-Diagramm auch die entsprechenden Dehnungen $\varepsilon_{\text {emaxi }}$ bekannt.

4. Mit den Werten $\sigma_{e \max i}$ und $\varepsilon_{e \max i}$ sowie mit $x_{\max }=$ konst. $=s / 2$ können nach 2.21 die zugehörigen Rissweiten bestimmt werden, woraus sich nach (67) der kritische Gelenkwinkel $\Theta_{k r}$ ergibt.

Beim Gebrauch der Formeln (139) beziehungsweise ( 104 ) stellt sich stets die Frage, was für Zahlenwerte für die Grössen $M$ und $Q$ einzusetzen sind. Beim Versagen der Zugarmierung liegen für die Bestimmung des Momentes die Ver- 
hältnisse günstig:

$$
\mathrm{M}=\mathrm{M}_{\mathrm{kre}}=\mathrm{F}_{\mathrm{e}} \cdot \boldsymbol{\beta}_{\mathrm{z}} \cdot \mathrm{y}
$$

Die entsprechende Querkraft hingegen kann nicht auf einfache Weise ermittelt werden, denn sie hängt von der momentanen Grösse des Momentes im benachbarten plastischen Gelenk und dieses wiederum - vor allem infolge der Verfestigung der Zugarmierung - vom dortigen Verformungszustand, das heisst, vom erreichten Gelenkwinkel, ab. Letzterer jedoch ist seinerseits eine Integralgrösse der Verformungen im gesamten Tragwerk. Man muss sich deshalb meist mit Näherungsüberlegungen helfen. Da die Kräfte in der Zugarmierung - und damit auch die Verformungen - in den Rissen neben dem kritischen Riss umso kleiner sind, je grösser die Querkraft, beziehungsweise je kleiner das Momenten-Schub-Verhältnis ist, genügt es, eine Querkraft einzusetzen, welche mit Sicherheit etwas grösser als die effektive ist. Dadurch wird der Winkel $\theta_{\mathrm{kr}}$ unterschätzt, das heisst, man befindet sich auf der sicheren Seite.

Die Querkraft $Q$ darf also nicht der normalen Traglastberechnung (siehe 4.1) entnommen werden. Um einen möglichst zutreffenden Wert für $Q \mathrm{zu}$ erhalten, muss vielmehr eine "modifizierte" Traglastberechnung durchgeführt werden. Bei dieser sind anstelle der plastischen Momente $M_{p}$ im kritischen Gelenk $M_{k r}$ nach ( 149 ) und in den übrigen Gelenken "modifizierte" plastische Momente $M_{p}$ ' nach ( 186 ) einzusetzen, wobei $\sigma_{e \max }$ geschătzt werden muss. Oder es kann in allen plastischen Gelenkes $M_{k r}$ angesetzt werden. Die aus den resultierenden Momentenflächen berechnete Querkraft stellt dann einen sicheren Wert dar, sofern jeweils zwischen zwei benachbarten plastischen Gelenken ein Momentennullpunkt liegt. Dies ist bei Stahlbetonbalken fast immer der Fall, da sich die plastischen Gelenke meist an den Stellen der stärksten Armierungen befinden. 


\subsection{Versagen des Betons}

Recht schwierig ist in einem Schubriss-Gelenk die rechnerische Erfassung des Versagens des Betons. Es sind hier vor allem zwei Arten zu unterscheiden:

a) Versagen des Betons im Steg

b) Versagen des Betons am Druckrand

Das Versagen der Biegedruckzone infolge Ueberbeanspruchung durch Schub hat Ursachen, die in den Abschnitten 3.34 und 3.35 behandelt werden. Hier wird vorausgesetzt, dass die Bügel keine plastischen Deformationen aufweisen und auch der Verbund der Längsarmierung genüge.

Während man auf Grund der üblichen Stahlbetonbiegetheorie geneigt ist, dem Versagen des Betons am Druckrand eine grosse Bedeutung zuzumessen, zeigen Versuche, dass dieses in einem Schubriss-Gelenk meist nicht wesentlich ist. Hingegen ist das Versagen des Stegbetons wichtiger, sodass zuerst darauf eingetreten wird.

\section{a) Versagen im Steg}

Im Steg eines Schubriss-Gelenkes sind erhebliche schiefe Druckkräfte vorhanden. Der Stegbeton erfährt zudem Deformationen durch die Rotation, das heisst, infolge der sich verändernden Neigung der Druckstreben, sowie durch kleine Schiebungen, welche infolge der - hier ausschliesslich elastischen - Dehnungen der Bügel entstehen.

Die Gefahr für ein Versagen des Stegbetons ist somit an der Wurzel der schiefen Druckstreben am grössten, wo deren Querschnitt am geringsten ist. Die massgebende Stelle liegt beim Rechteckquerschnitt am Ende der Risse. Ist ein Druckflansch vorhanden, so befindet sie sich am Uebergang vom Steg zum Flansch. In Anlehnung an Bild 33 kann die massgebende Druckfläche $F_{D}$ senkrecht zur Axe einer schiefen Druckstrebe wie folgt ausgedrückt werden:

$$
F_{D} \cong b_{D} \cdot s \cdot \cos \delta_{i, i+1} \cdot \frac{a_{D}}{h}
$$


Die Breite der Druckfläche (senkrecht zur Bildebene), $b_{D}$, und der Abstand der Druckfläche vom Druckrand, ${ }^{D}$, sind wie folgt einzusetzen:

Rechteckquerschnitt: $\quad b_{D}=b, \quad a_{D}=z$.

Querschnitt mit Druckflansch, Flanschhöhe $\mathrm{h}_{\mathrm{F}}$ :

$\begin{array}{lll}h_{F}>z: & b_{D}=b_{0}, & a_{D}=h_{F} . \\ h_{F}<z: & b_{D}=b_{0}, & a_{D}=z .\end{array}$

Mit dem Rissabstand

$$
\mathbf{s}=h \cdot\left(\operatorname{tg} \delta_{i, i+1}-\operatorname{tg} \delta_{i}\right)
$$

wird

$$
F_{D} \cong b_{D} \cdot a_{D} \cdot \cos \delta_{i, i+1} \cdot\left(\operatorname{tg} \delta_{i+1}-\operatorname{tg} \delta_{i}\right)
$$

Obschon also die schiefen Druckspannungen nicht die alleinige Beanspruchung darstellen, kann deren Grösse für die Gefahr eines Versagens des Stegbetons doch als massgebend angesehen werden. Die mittlere Druckspannung $\sigma_{b D}$ wird auf Grund der Gleichungen ( 145 ), ( 147b) und ( 152 ):

$$
\sigma_{b D} \cong \frac{B_{45}}{b_{D} \cdot a_{D}} \cdot \frac{1}{(1-z / 2 h) \cdot \cos ^{2} \delta_{i, i+1}}
$$

$\sigma_{\text {bD }}$ kann auch als Funktion der nominellen Schubspannung $\tau$ ausgedrückt werden. Mit den Gleichungen ( 121 ), ( 132a ), ( 134 ), ( 142 ) und ( 144 ) wird aus ( 153 ) für einen Querschnitt mit Druckflansch:

$$
\sigma_{b D} \cong \frac{\tau-\tau_{1}}{\eta \cdot \cos ^{2} \delta_{i, i+1}} \cdot\left[\frac{h}{a_{D}}\right] .
$$


Aus ( 153 ) beziehungsweise ( 154 ) ist ersichtlich, dass $\sigma_{\mathrm{bD}}$ umso grösser wird, je kleiner $\cos \delta_{i, i+1}$ ist. Die grösste Druckspannung tritt daher erwartungsgemäss in der flachsten, das heisst äussersten Druckstrebe auf. Da nach ( 125 ) die Neigung des flachsten Biegeschubrisses maximal etwa $\delta_{n} \cong 52^{\circ}$ beträgt, ergibt sich stets $\cos ^{2} \delta_{i, i+1} \geq \sim 0,4$.

Interessant ist ein Vergleich mit den in schubbeanspruchten Trägern ausserhalb von Biegeschubrissen, das heisst im sogenannten "Diagonalschubbereich" (vergleiche [9] ), auftretenden schiefen Druckspannungen. Dort verlaufen die Schubrisse ungefähr parallel, und man erhält bekanntlich für ein Ständerfachwerk unter der Annahme, dass die gesamte Querkraft durch vertikale Zugstäbe (Biigel) und durch um $45^{\circ}$ geneigte Druckstreben übertragen wird, eine Druckspannung von

$$
\sigma_{b D} \cong 2 \tau \text {. }
$$

Dasselbe Resultat lässt sich auch aus ( 154 ) herleiten. Vernachlässigt man $\tau_{1}$ gegenüber $\tau$ und setzt $\eta=1,0$, so wird für $\delta_{i, i+1}=45^{\circ}$ :

$$
\sigma_{b D} \cong 2 \tau \cdot\left(\frac{h}{a_{D}}\right)
$$

Für eine von oben bis unten gleichbleibende Breite der Druckstrebe ist $h / a_{D}=1$, womit ebenfalls $G_{\mathrm{bD}} \cong 2 \tau$ resultiert.

Natürlich stellt ( 154 ) nur eine grobe Abschätzung der tatsächlich auftretenden Druckspannungen dar und enthält verschiedene Unzulänglichkeiten. So verlaufen etwa Biegeschubrisse noch näher zum Druckrand hin, als $a_{D}=z$ dies beim Rechteckquerschnitt berücksichtigt. Oder die Breite der Druckstrebe verändert sich nicht immer linear zum Abstand von der Druckkante, da unter anderem auch die Auflagerbreite einen Einfluss hat. Trotzdem zeigen die Gleichungen ( 154 ) beziehungsweise ( 156 ) die Grössenordnung befriedigend an. Man erkennt, dass in einem Schubriss-Gelenk - und auch allgemein im Bereich von Biegeschubrissen, die auf die Stelle einer direkten Krafteinleitung hin laufen - ganz beträchtliche Druckspannungen auftreten können. Der Vergleich mit ( 155 ) ergibt, dass diese 
Druckspannungen - je nach dem Verhältnis $h / \oint_{D}$ - meist ein Mehrfaches derjenigen betragen, welche sich aus der üblichen Fachwerkanalogie ergeben, beziehungsweise im Diagonalschubbereich etwa auftreten.

Bei der Beurteilung von ( 154 ) beziehungsweise ( 156 ) scheinen auf den ersten Blick Rechteckquerschnitte mit schwacher Zugarmierung besonders gefährdet, da dort $a_{D}=z$ klein, beziehungsweise $h / a_{D}$ gross ist. Bei solchen Gelenken sind jedoch meistens die Neigung $\delta_{n}$ des flachsten Biegeschubrisses und auch die Schubbeanspruchung verhältnismässig gering, was den ungünstigen Einfluss von $a_{D}=z$ auf $\sigma_{b D}$ kompensiert. Sehr grosse Druckspannungen $\sigma_{b D}$ treten eher in Gelenken mit verhältnismässig dünnen Druckflanschen auf.

Am Fuss der Druckdiagonalen erleidet der Beton Deformationen infolge schiefem Druck und Rotation. Zudem bewirken die (elastischen) Verlängerungen der Bügel gewisse Schiebungen. Die aufnehmbare schiefe Druckspannung nach (153) beziehungsweise ( 154 ) wird deshalb vorsichtshalber wie folgt begrenzt:

$$
\sigma_{\mathrm{bD}}=0,8 \mathrm{\beta}
$$

Ueberschreitet also $G_{\mathrm{bD}}$ in der flachsten Druckdiagonalen $80 \%$ der Prismenfestigkeit, so ist mit einem Versagen des Betons zu rechnen.

In Vorschriften und Vorschlägen für die Schubbemessung wird meist eine sogenannte "obere Schubspannungsgrenze" angegeben, das heisst, die Schubbeanspruchung eines Querschnittes darf eine bestimmte Grösse nicht überschreiten. Dadurch sollen Schubdruckbrüche im Steg vermieden werden. In [9] wurden die Schubspannungen wie folgt begrenzt (nur vertikale Bügel, $\tau_{1}$ nach 3.11):

Normale Abstände $(t \leq h / 2$, jedoch $t \leq 30 \mathrm{~cm})$ :

$$
\tau=4 \tau_{1}
$$

Enge Abstände $(t=h / 3$, jedoch $t=20 \mathrm{~cm})$ :

$$
T=5 \tau_{1}
$$


Diese Regeln sind im Lichte der vorangehenden Untersuchungen bei der Schubbemessung im Bereiche eines plastischen Gelenkes unbedingt einzuhalten. Ein vorsichtiger Konstrukteur wird jedoch, solange nicht umfassende Versuchsresultate vorliegen, eine eher noch engere, das heisst, möglichst fein verteilte Schubarmierung einlegen und mit der Schubbeanspruchung wenn möglich trotzdem die Grenze ( 158 ) nicht überschreiten.

Ein schwieriges Problem stellt sich mit der Frage, wie der im Moment des Versagens des Stegbetons, beziehungsweise beim Erreichen von $G_{b D}=0,8 \beta$ vorhandene kritische Gelenkwinkel $\Theta_{\mathrm{krbD}}$ berechnet werden soll. Wie aus (153) beziehungsweise ( 154 ) hervorgeht, ist unter den früher getroffenen Voraussetzungen die Schubspannung $\tau$ die einzige der für $G_{b D}$ massgebenden Grössen, welche sich während der Rotation verändert. Um $\Theta_{k r b D}$ bestimmen zu können, muss somit im betrachteten Gelenk das Anwachsen des Gelenkwinkels mit zunehmender Querkraft, also die Funktion $\Theta(Q)$, bekannt sein. Ein möglicher Verlauf ist generell in Bild 35 dargestellt, vorerst unter Voraussetzung einer bis zum Versagen der Zugarmierung reichenden Rotationsfähigkeit. Am Anfang ist bei stark

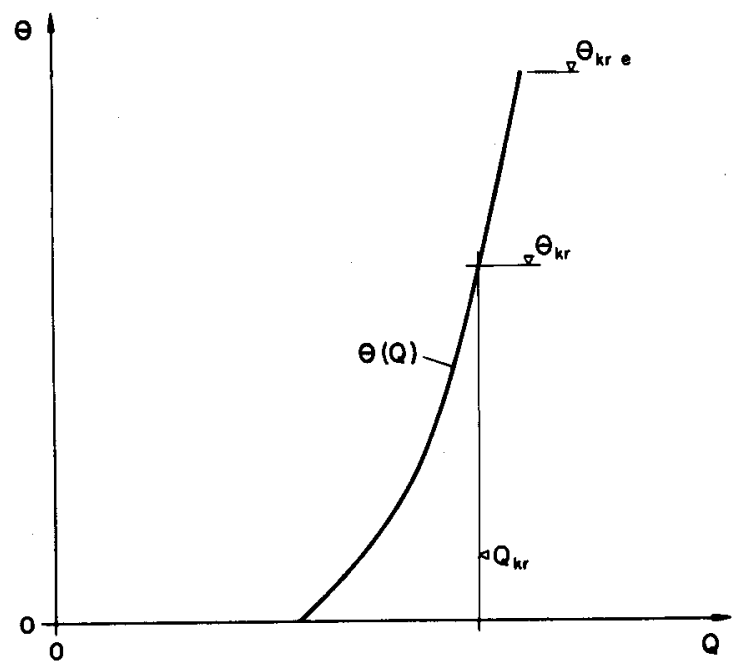

Bild 35

Funktion $\Theta(Q)$ : Bestimmung von $\Theta_{k r}$ anhand von $Q_{k r}$ aus Versagenskriterium 
zunehmender Querkraft der Rotationswinkel gering, später wächst er stärker an. Wird aus ( 153 ) oder ( 154 ) die für $\sigma_{b D}=0,8 \beta$ vorhandene "kritische" Querkraft $Q_{\text {krbD }}$ berechnet, so kann aus $\Theta(Q)$ der entsprechende kritische Gelenkwinkel $\Theta_{\mathrm{kr} \mathrm{bD}}$ bestimmt werden.

Grundsătzlich besteht die Möglichkeit, die Funktion $\Theta(Q)$ punktweise zu berechnen. Ein entsprechendes Verfahren wird im Abschnitt 4.22 b) angegeben. Dieses ist an sich bereits ziemlich kompliziert und aufwendig. Da bei der Bestimmung von $\Theta_{\mathrm{krbD}}$ ein "schleifender Schnitt" entsteht, ist es ausserdem nötig, bei der Berechnung von $\Theta(Q)$ anstelle der plastischen Momente $M_{p}$ "modifizierte" plastische Momente $M_{p}{ }^{\prime}$ (siehe $4.32 \mathrm{~b}$ ), $M_{p}<M_{p}^{\prime}=M_{k r}$ ) einzusetzen, damit das Resultat einigermassen zutreffend ist. Diese Werte $M_{p}{ }^{\prime}$ in den Gelenken müssen vorerst geschätzt werden, beziehungsweise $\Theta(Q)$ kann nur durch eine recht aufwendige Iteration erarbeitet werden. Zudem stellt das Kriterium ( 157 ) für das Versagen des Stegbetons natürlich eine ziemlich willkürliche Festlegung dar. In Wirklichkeit wird die zum Versagen führende schiefe Druckspannung stets mehr oder weniger von $0,8 \beta$ abweichen. Ebenso können die Annahmen, welche den Ausdrücken ( 153 ) beziehungsweise ( 154 ) zugrundeliegen, nicht genau den tatsächlichen Gegebenheiten entsprechen. Bei einer Bemessung wird man sich auf Grund all dieser Schwierigkeiten bemühen, allzu grosse schiefe Druckspannungen, das heisst, vor allem allzu hohe Schubbeanspruchungen, zu vermeiden. Eine obere Grenze kann stets aus einer Traglastberechnung gewonnen werden, bei welcher in den plastischen Gelenken anstelle von $M_{p}$ die Momente $M_{k r e}$ angesetzt werden (siehe 3.32). Beträgt die dermassen berechnete schiefe Druckspannung weniger als $0,8 \beta$, so ist kein vorzeitiges Versagen des Stegbetons zu erwarten. Ist dies jedoch nicht der Fall, so sollte nach Möglichkeit durch die Anordnung eines dickeren Steges diese Gefahr gebannt werden.

Handelt es sich um die Nachrechnung eines bestehenden Tragwerkes, bei welchem $\sigma_{b D}$ als kritisch erkannt wurde, so wird es infolge der vorerwähnten Umstände auch mit sehr grossem Aufwand für die Iterationsberechnung von $\Theta(Q)$ - kaum gelingen, $\Theta_{\mathrm{krbD}}$ zuverlăssig vorauszusagen.

Für einen bestimmten bekannten Gelenkwinkel - hier also $\Theta_{k r b D}$ - können die entsprechenden Verformungen in der Zugzone auf iterativem Wege berechnet werden 
Vorerst ist im steilsten und breitesten Gelenkriss die maximale Stahldehnung $\varepsilon_{\text {e max } 1}$ beziehungsweise die Rissweite $w_{1}$ anzunehmen. Davon ausgehend sind die andern Risswinkel nach dem unter 3.32 angegebenen Verfahren zu berechnen. Die Kontrolle

$$
\sum_{1}^{n} \omega=\theta_{k r b D}
$$

zeigt dann, ob die Rechnung mit andern Ausgangswerten $\varepsilon_{\mathrm{e} \max 1}$ beziehungsweise $w_{1}$ wiederholt werden muss.

\section{b) Versagen am Druckrand}

Der generelle Verlauf der Betonstauchungen längs des Druckrandes eines Schubriss-Gelenkes ist in Bild 36 dargestellt. Er entspricht den in den Berichten [7] und [8] wiedergegebenen Messungen an 10 Schubriss-Gelenken. Der Verlauf ist dacurch gekennzeichnet, dass unmittelbar beim Rande der Krafteinleitung sehr hohe Stauchungen auftreten, die jedoch mit zunehmender Entfernung von demselben stark abfallen. Bei den zitierten Versuchen wurden sogar etwa im Abstand h/2 vom Rande der Krafteinleitung teilweise bereits Zugverformungen festgestellt.

Dass grosse Betonstauchungen nur in einem kleinen Bereich neben der Krafteinleitung auftreten können, zeigt ein Blick auf unser Modell des Schubriss-Gelenkes (Bilder 31 und 32). Der unterhalb des flachsten Biegeschubrisses n gelegene, keilförmige Balkenteil weist vorerst eine recht grosse Höhe auf, die dann gegen die Krafteinleitung zu stark abnimmt. Für die Beanspruchungen aus der Druckkraft $C_{n}$ bleibt schliesslich nur noch ein kleiner Querschnitt zur Verfügung, der ausserdem durch die Rotation noch stark deformiert wird. Die Betonstauchungen vergrössern sich somit keineswegs - wie es nach der üblichen Biegelehre erwartet werden könnte - entsprechend dem Momentenverlauf, sondern es ergibt sich eine starke Steigerung erst unmittelbar vor dem Rande der Krafteinleitung.

Bei den Versuchen [7] und [8] begannen sich im Laufe der Rotation am Druckrand neben der Krafteinleitung kleine Betonlamellen abzulösen. Diese waren 

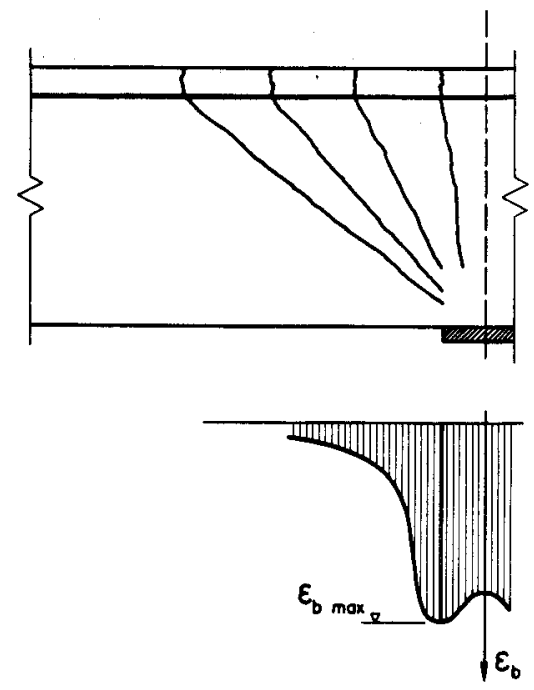

\section{Bild 36}

Genereller Verlauf der Betonstauchungen am Druckrand eines Schubriss-Gelenkes

vorerst nur wenige $\mathrm{mm}$ dick und 1 bis $2 \mathrm{~cm}$ breit. Schliesslich war bei einer Balkenhöhe von $38 \mathrm{~cm}$ längs dem Rande der Krafteinleitung ein etwa $3 \mathrm{~cm}$ breiter und $1 \mathrm{~cm}$ tiefer Zerstörungsbereich vorhanden. Ueber denselben hinweg wurde die Randverformung gemessen. Die beim Beobachten der ersten Ablösungen festgestellten "Betonstauchungen" betrugen jeweils etwa 3,5 bis $5 \%$. Sie wuchsen dann teilweise bis über $8 \% 0$ an. Trotzdem ergaben sich keine Anzeichen dafür, dass die Funktionstüchtigkeit der Biegedruckzone beeinträchtigt worden wäre. Bei keinem der Balken trat ein Versagen im Gelenk infolge der beschriebenen lokalen Zerstörung der Randfaser ein; dieses hatte stets andere Ursachen.

Es mag sein, dass auf die Biegedruckzone die an den Bügeln aufgehängte Druckarmierung von $\mu^{\prime} \cong 0,35 \%$ eine umschnürende Wirkung ausübte. Auch ist deren 
Dübelwirkung nicht ausser acht zu lassen. Ob sich jedoch unarmierte Biegedruckzonen wesentlich ungünstiger verhalten hätten, ist schwer zu entscheiden. Darüber müssten systematische Versuche durchgeführt werden. Zumindest für Verhältnisse, welche etwa denjenigen der zitierten Versuche entsprechen, kann somit festgehalten werden:

In einem Schubriss-Gelenk treten am Druckrand nur über einem verhältnismässig kleinen Bereich hohe Betonstauchungen auf. Die entsprechenden Zerstörungen der Randfaser führen daher nicht, oder erst nach sehr grosser Rotation, zu einem Versagen im Gelenk.

Aus dieser Erkenntnis darf jedoch nicht der Schluss gezogen werden, dass in einem Schubriss-Gelenk die Grösse der Biegedruckkraft und daher der Zugarmierungsgehalt beliebig gross sein dïrfen. Im Abschnitt $2.53 \mathrm{~d}$ ) wurde gezeigt, dass der dort definierte Grenzarmierungsgehalt $\mu_{\mathrm{Gr}}$ nach ( 112 ) es erlaubt, in einem Biegeriss-Gelenk die sogenannte Sprödbruchgefahr abzuschätzen. Auch in einem Schubriss-Gelenk soll darauf geachtet werden, dass der entsprechende Wert $\mu_{\mathrm{Gr}}$ nicht erreicht wird.

Im folgenden wird noch eine - allerdings nur auf den Versuchen [7] und [8] basierende - Möglichkeit gezeigt, wie in einem Schubriss-Gelenk die Grössen ordnung der maximalen Betonstauchung $\varepsilon_{b \max }$ am Rande neben der Krafteinleitung (Mittelwert über eine Lănge von etwa $h / 10$ ) abgeschätzt werden kann. Es wurde festgestellt, dass $\varepsilon_{b \max }$ stets etwa linear mit dem Rotationswinkel anwächst:

$$
\varepsilon_{\mathrm{b} \max }=\mathbf{k}_{\mathrm{b}} \cdot \boldsymbol{\theta}
$$

Wird die Konstante $k_{b}$ proportional zur Druckkraft in der Biegedruckzone $\left(C_{n}=\right.$ $z_{n} \cong \sigma_{f} \cdot F_{e}$ ) und umgekehrt proportional zur Betonfestigkeit angesetzt, was entspricht

$$
k_{b}=k_{b}^{\prime} \mu \cdot \frac{\sigma_{f}}{\beta_{w}} \text {, }
$$


und $k_{\mathrm{b}}$ aus [7] und [8] $\mathrm{zu} 3,1$ ermittelt, also

$$
\varepsilon_{b \max }=3,1 \cdot \mu \cdot \frac{\sigma_{f}}{\beta_{w}} \cdot \Theta,
$$

so ergibt sich eine mittlere quadratische Abweichung der rechnerischen zu den gemessenen Grössen $\varepsilon_{b \max }$ von etwa $\pm 20 \%$. Wie oben dargelegt, können Werte $\varepsilon_{\mathrm{b} \max }$ von $8 \% \mathrm{o}$ und mehr erreicht werden.

\subsection{Versagen der Bügelarmierung}

Eine weitere Bruchursache beziehungsweise ein Kriterium für die Erschöpfung der Deformationsfăhigkeit eines Schubriss-Gelenkes kann das Versagen der Bügelarmierung sein. In diesem Falle wird der Bruchvorgang eingeleitet durch plastische Verformungen der Bügel (vergleiche [8]). Dadurch vergrössern sich die Stegdeformationen erheblich, sodass Schiebungen sowie Lockerungen des Betongefüges in den Druckstreben und der Biegedruckzone auftreten. Bei einer weiteren Erhöhung der Querkraft können die Bügel keine oder nur noch geringe zusătzliche Krăfte übernehmen. Demzufolge wird die Biegedruckzone überbelastet, was rasch zu deren Zerstörung führt.

Ein Bruch infolge Versagen der Bügelarmierung tritt somit ein, wenn die Bügel bis etwa zur Streckgrenze beansprucht sind. Allgemein kann die Resultierende der Bügelkräfte in einem $45^{\circ}$-Riss nach Bild 32 wie folgt ausgedrückt werden:

$$
B_{45}=F_{B} \cdot \frac{(h-z / 2)}{t} \cdot G_{B}
$$

$F_{B}$ ist der Querschnitt eines Bügelelementes, $t$ der Bügelabstand und $\sigma_{B}$ die mittlere Bügelspannung. Erreicht $\sigma_{B}$ die Streckgrenze $\sigma_{s B}$, so ergibt sich unter Vernachlässigung von $\mathrm{z} / 2$ :

$$
\mathbf{Q}_{\mathbf{B}}=\mathbf{F}_{\mathbf{B}} \cdot \frac{\mathbf{h}}{\mathbf{t}} \cdot \sigma_{\mathbf{S B}}
$$


$Q_{B}$ kann wie in [9] als "Schubwiderstand der Bügelarmierung" bezeichnet werden. Damit wird aus Gleichung ( 141 ):

$$
Q_{B}=\mathbf{Q}-\mathbf{K}_{45}
$$

In Abschnitt 3.22 wurde ferner gezeigt, dass für den durch die Biegedruckzone übertragenen Anteil an der Querkraft im Bereich plastischer Verformungen der Zugarmierung der "Schubwiderstand der Biegedruckzone" $Q_{C}$ nach Gleichung ( 142 ) eingesetzt werden kann. Somit ist mit einem Versagen der Bügelarmierung $\mathrm{zu}$ rechnen, sobald bei zunehmender Querkraft $Q$ die folgende Gleichung erfuillt wird:

$$
\mathbf{Q}_{\mathbf{B}}=\mathbf{Q}-\mathbf{Q}_{\mathbf{C}}
$$

Wie aus den Versuchen [7] und [8] geschlossen werden kann, gibt diese Gleichung eine recht gute Grundlage zur Beurteilung der diskutierten Bruchgefahr. Die Vernachlässigung von $z / 2$ bei der Definition von $Q_{B}$ wird ungefähr kompensiert durch den eher vorsichtigen Ansatz für $Q_{C}$.

Die Bügelarmierung sollte stets so bemessen werden, dass deren Versagen nicht vor demjenigen des Betons oder der Zugarmierung erfolgt. Die entsprechende Bemessungsbedingung lautet:

$$
Q_{B}+Q_{C} \geq Q\left(M_{k r}\right)
$$

Es muss somit der Dimensionierung der Bügel eine genügend grosse Querkraft zugrunde gelegt werden. $Q$ darf, ähnlich wie unter 3.32 , nicht der normalen Traglastberechnung nach 4.1 entnommen werden, sondern es muss eine "modifizierte" Traglastberechnung durchgeführt werden. Bei dieser sind in den plastischen Gelenken anstelle von $M_{p}$ geschätzte beziehungsweise iterativ berechnete Werte $M_{p}$ ' anzusetzen. Ein sicherer Wert für $Q$ ergibt sich natürlich wiederum mit $M_{p}^{\prime}=M_{k r e}$ in allen plastischen Gelenken. Dieser kann allerdings - je nach Ausmass der Verfestigung der Zugarmierung und je nach Ursache des Versagens im kritischen Gelenk - wesentlich höher als der tatsächliche liegen und daher zu 
einer Ueberdimensionierung der Bügelarmierung führen.

Bei der Nachrechnung eines bestehenden Tragwerkes, bei welchem die Stärke der Bügelarmierung als ungenügend erkannt wurde, bleibt nichts anderes übrig, als den Zusammenhang zwischen dem Gelenkwinkel und der Querkraft, das heisst, die Funktion $\Theta(Q)$, mit $M_{p}{ }^{\prime}$ in den plastischen Gelenken auf iterativem Wege $z u$ berechnen (vergleiche Bild 35). Dabei, sowie bei der Ermittlung von $\Theta_{k r ~ B}$, ergeben sich jedoch analoge Probleme, wie sie im Abschnitt 3.33 a) ausführlich besprochen wurden. Die dortigen Schlussfolgerungen treffen hier weitgehend zu: Auch mit sehr grossem Rechenaufwand für die Ermittlung von $\Theta(Q)$ wird es kaum gelingen, $\Theta_{\mathrm{kr} \mathrm{B}}$ zuverlässig vorauszusagen.

\subsection{Versagen des Verbundes der Zugarmierung}

In den Abschnitten 3.31 beziehungsweise 2.41 b) ist dargelegt worden, auf welche Weise eine Veränderung der Zugkraft in der Längsarmierung durch Verbundspannungen hervorgerufen wird. Analog zu Gleichung ( 73 ) gilt im Bereich zwischen zwei Biegeschubrissen (vergleiche Bild 34):

$$
\Delta z_{i, i+1}=\int_{0}^{s} \tau_{v}(x) \cdot \sum u \cdot d x
$$

Bei festgelegten geometrischen Abmessungen liegen der Verschiebungsnullpunkt beziehungsweise die Nullstelle der Verbundspannungen umso näher beim flacheren Biegeschubriss, das heisst, die Strecke $q_{i, i+1}$ ist umso grösser, je geringer die Verbundspannungen im Verhältnis zur Kraft $\Delta \mathrm{z}_{\mathbf{i}, \mathbf{i}+1}$ sind. $\Delta \mathrm{z}_{\mathbf{i}, \mathbf{i}+1}$ wächst bei zunehmender Belastung nach Gleichung ( 140 ) an, nämlich proportional zur Grösse $B_{45}$, welche nach ( 144 ) stark abhängig von der Querkraft $Q$ ist. Sind die Verbundspannungen verhältnismässig gering, beziehungsweise die Differenzkraft $\Delta \mathrm{Z}_{i, i+1}$, das heisst die Schubbeanspruchung, gross, so kann q stark anwachsen und bald zu $s / 2$ werden. Dies bedeutet, dass sich auf der ganzen Strecke $s$ die Zugarmierung relativ zum Beton in derselben Richtung verschiebt. Schliesslich tritt der Fall ein, dass bei gleitender Zugarmierung die Kraft $\Delta \mathbf{Z}_{\mathbf{i}, \mathbf{i}+1}$ nicht 
mehr weiter zunehmen kann oder - je nach Verbundgesetz - sogar abnimmt. Für grössere Werte $B_{45}$ beziehungsweise $Q$ ist dann Gleichung ( 140 ) nicht mehr gültig. Ein weiterer Zuwachs an Querkraft kann bei gleitender Längsarmierung nicht mehr durch die Bügel, sondern höchstens noch durch die Biegedruckzone aufgenommen werden. Diese wird jedoch meist bald uberbeansprucht und versagt. Es tritt somit ein Bruch ein, dessen Ursache im Versagen des Verbundes der Längsarmierung begründet ist.

Um die Gefahr für das beschriebene Versagen abzuschätzen, wird am besten eine konstante Verbundspannung angenommen:

$$
\tau_{v}(v) \cong \text { konst. } \quad=\tau_{v}^{*}
$$

Wie früher (2.21) gezeigt wurde, trifft diese Annahme für Rundstähle und Riegeltorstahl recht gut zu. Bei Rippenstählen müssen mittlere Verbundspannungen geschätzt werden. Praktische Werte können aus den entsprechenden Verbundgesetzen oder - bei deren Fehlen - zum Beispiel aus den Gleichungen (65) mit $\tau_{\mathbf{v}}{ }^{*} \cong$ $\tau_{v R}$ ermittelt werden. Dabei sind allgemein bei oben liegenden Stäben, also zum Beispiel über Zwischenstützen von Durchlaufträgern, wegen dem bekannten Absetzen des Betons, eher vorsichtige, das heisst kleinere, Werte anzunehmen. Damit kann die Strecke $q_{i, i+1}$ nach Gleichung $(75)$ berechnet werden:

$$
q_{i, i+1}=\frac{z_{i, i+1}}{2 \tau_{v}{ }^{*} \cdot \pi \cdot \Sigma d}
$$

Eine Gefahr für das Versagen des Verbundes der Längsarmierung ist dann vorhanden, wenn

$$
q_{i, i+1}=s / 2
$$

ist.

Es stellt sich hier wiederum die Frage, welche Zahlenwerte bei der Berechnung von $\Delta Z_{i, i+1}$ nach ( 140 ) für die Querkraft $Q$ einzusetzen sind. Diese werden vor- 
sichtigerweise wiederum einer "modifizierten" Traglastberechnung entnommen, wie sie in Abschnitt 3.32 beschrieben worden ist und bei der in allen Gelenken $M_{p}^{\prime}=M_{k r}$ angesetzt wird.

Ein äusserst schwieriges Unterfangen dưrfte es sein, den Rotationswinkel beim Versagen des Verbundes, $\Theta_{\mathrm{krv}}$, berechnen zu wollen. Dazu wäre es - ăhnlich wie beim Versagen des Betons im Steg und beim Versagen der Bügelarmierung nötig, die Funktion $\Theta(Q)$ zu kennen, deren aufwendige Ermittlung unter 3.33 a) behandelt wurde. In $\Theta(Q)$ wäre dann die aus der Kombination von ( 168$),(75)$ und ( 140 ) zu ermittelnde kritische Querkraft $Q_{k r ~ v}$ einzusetzen (vergleiche Bild 35). Da es sich jedoch vor allem wegen ( 75 ) um eine im Verhălnis zur "Steilheit" der Funktion $\Theta(Q)$ grobe Schătzung handelt, käme einem derart bestimmten Wert $\Theta_{\mathrm{krv}}$ nur eine äusserst beschränkte Aussagekraft zu.

Glücklicherweise ist es jedoch so, dass die an den meistverwendeten, gut profilierten Armierungsstählen entwickelten Verbundspannungen recht hoch sind. Sie gentigen meist auch bei hohen Schubbeanspruchungen, welche die im Abschnitt 3.33 a) gemäss [9] zitierten oberen Grenzen erreichen, um das Versagen des Verbundes der Längsarmierung zu verhindern. Man kann es somit bei einer Kontrolle der Grössenordnungen bewenden lassen. Bei Rundstählen hingegen muss mit dem erwăhnten Versagen stets gerechnet werden, sobald höhere Schubbeanspruchungen auftreten. Bei solchen sind jedoch Rundstähle bekanntlich denkbar ungeeignet, unabhängig von einer allfalligen Anordnung im Bereiche plastischer Gelenke.

\subsection{Weitere Aspekte}

Wie die vorangehenden Darlegungen zeigen, ist in Schubriss-Gelenken die Berechnung des kritischen Gelenkwinkels in vielen Fällen äusserst kompliziert und aufwendig, wobei das Resultat erst noch grosse Unsicherheiten aufweisen kann. Um jedoch die Verhältnisse genügend beurteilen zu können, müssen noch einige zusätzliche Aspekte beachtet werden. 


\subsection{Verlauf der Kraft in der Zugarmierung}

In einem Biegeschubriss des Gelenkes kann die Kraft in der Zugarmierung nach ( 139 ) berechnet werden. Die Differenz der Zugkräfte in zwei benachbarten Rissen ergibt sich aus ( 140 ). Zur Diskussion, und um die Zusammenhänge noch besser zu überblicken, jedoch kaum für den praktischen Gebrauch, können in diesen Gleichungen die folgenden Vereinfachungen vorgenommen werden:

$$
\begin{aligned}
& y \cong h \\
& z \cong 0 \\
& \eta \cong 1 \\
& b_{a} \cong 0
\end{aligned}
$$

Aus ( 139 ) und ( 140 ) erhält man:

$$
\begin{aligned}
Z_{i} & =Q \cdot\left[\frac{M_{I}}{Q h}-\frac{B_{45}}{Q} \cdot \frac{\operatorname{tg}^{2} \delta_{i}}{2}\right] \\
\Delta Z_{i, i+1} & =-B_{45} \cdot\left[\frac{\operatorname{tg}^{2} \delta_{i}-\operatorname{tg}^{2} \delta_{i+1}}{2}\right]
\end{aligned}
$$

Nach ( 170 ) ergibt sich die Zugkraft $\mathrm{Z}_{\mathbf{i}}$ durch die Multiplikation der Querkraft mit einem Faktor, welcher aus der Differenz zwischen dem Momenten-SchubVerhältnis und einem Glied besteht, das die Einflüsse des Quotienten $B_{45} / Q$ und der Neigung des betreffenden Biegeschubrisses erfasst. Die Zusammenhänge sind aus Bild 37 ersichtlich. Die Darstellung betrifft ausdrücklich nur die Zugkraft in den Rissequerschnitten, das heisst der Einfluss des Verbundes auf den Verlauf der Zugkraft zwischen den Rissen wurde nicht berücksichtigt.

Die Grösse des Quotienten $B_{45} / Q$ hängt einerseits ab vom Beanspruchungsgrad der Zugarmierung. Weist diese noch keine plastischen Deformationen auf, so ist $B_{45} / Q$ oft noch recht klein, insbesondere bei Rechteckquerschnitten (vergleiche 3.22). 'Ist die Zugarmierung jedoch stark plastisch gedehnt, so kann ( 144 ) verwendet werden. Somit hängt $B_{45} / Q$ andererseits auch von der absoluten 


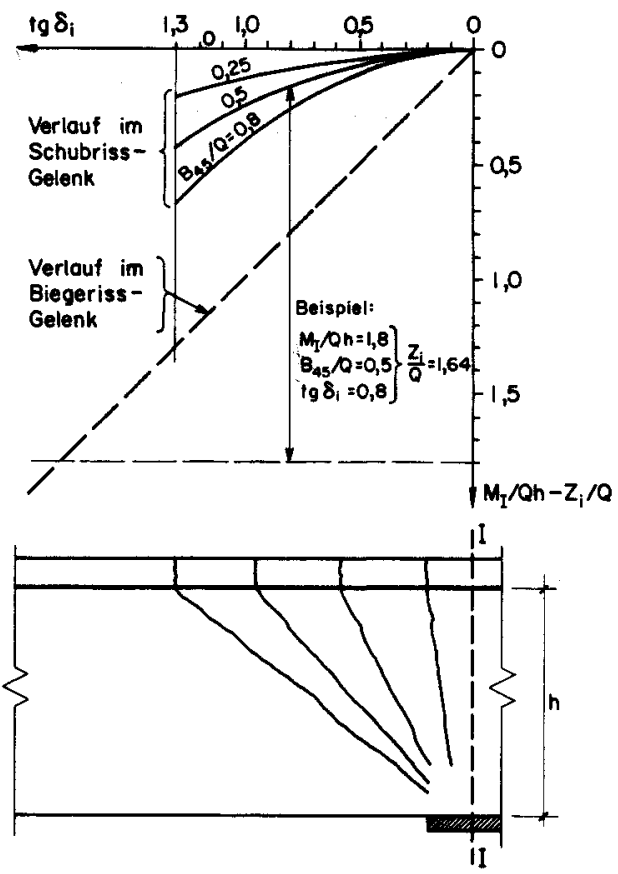

Bild 37

Verlauf der Zugkraft der Zugarmierung in den Rissequerschnitten (Einfluss des Verbundes auf den Verlauf zwischen den Rissen nicht berücksichtigt)

Grösse der Querkraft $Q$ ab. In Bild 37 wurde daher der Zugkraftverlauf im Gelenk für verschiedene Werte $B_{45} / Q$ dargestellt. Zum Vergleich wurde ausserdem noch der Zugkraftverlauf in einem entsprechenden Biegeriss-Gelenk gestrichelt eingetragen:

$$
Z_{\mathbf{i}}=\mathbf{Q} \cdot\left[\frac{\mathrm{M}_{\mathrm{I}}}{\mathrm{Qh}}-\operatorname{tg} \delta_{\mathbf{i}}\right]
$$

In ( 172 ) charakterisiert tg $\delta_{i}$ nicht mehr die Neigung eines Biegeschubrisses, sondern den Abstand des betrachteten Biegerisses von der Stelle des maximalen Momentes (Schnitt I - I). 
Aus Bild 37 erkennt man, wie verhältnismässig gering die Abnahme der maximalen Zugkraft in einem Schubriss-Gelenk ist. Sind keine Bügelkräfte vorhanden, so ist die Zugkraft in sämtlichen Biegeschubrissen dieselbe. Aber selbst beim maximal etwa möglichen Wert $B_{45} / Q=0,8$ ist die Abnahme noch nicht die Hälfte derjenigen, wie sie in einem entsprechenden Biegeriss-Gelenk auftreten wiirde, wo die Zugkraft in den Biegerissen affin zur Momentenfläche verläuft.

\subsection{Verlauf der Dehnungen in der Zugarmierung}

Für die Berechnung der kritischen Gelenkwinkel, beziehungsweise ganz allgemein zur Beurteilung der Rotationsfähigkeit, ist der Verlauf der Dehnungen $\varepsilon_{\text {e maxi }}$ in den Biegeschubrissen wesentlich. Als massgebende Grössen kommen somit zum Verlauf der Kraft in der Zugarmierung noch die Eigenschaften des betreffenden Spannungs-Dehnungs-Diagrammes - insbesondere in dessen plastischem Bereich - hinzu.

In einem Schubriss-Gelenk sind meistens ganz ansehnliche Armierungsgehalte und dementsprechend eher dickere Stabdurchmesser nötig, damit grössere Schubbeanspruchungen entstehen. Dadurch ergeben sich verhältnismässig kleine Rissabstände und grosse Verbundkoeffizienten. Die Gleichmassdehnung ist daher für die Gefahr eines Versagens der Zugarmierung nicht von derselben Bedeutung wie in einem Biegeriss-Gelenk. Hingegen haben die Verfestigungseigenschaften - ähnlich wie im Biegeriss-Gelenk - einen wesentlichen Einfluss auf den Verlauf der Dehnungen $\varepsilon_{\text {emaxi }}$ in den Rissequerschnitten des Gelenkes. Die Zusammenhänge können mit Hilfe des idealisierten Spannungs-DehnungsDiagrammes Bild 19 sowie der folgenden Beziehung erläutert werden:

$$
\frac{\varepsilon_{e \max i}}{\varepsilon_{e \max I}}=1-\frac{1}{\varepsilon_{e \max I}} \cdot \frac{B_{45}}{E_{v} F_{e}} \cdot \frac{\operatorname{tg}^{2} \delta_{i}}{2}
$$

In Bild 38 stellen die ausgezogenen Linien den Verlauf der Dehnungen $\varepsilon_{\text {e } \max i}$ der Zugarmierung in den Biegeschubrissen für zwei verschiedene Verfestigungsmoduli $E_{v}$ dar, und zwar ausgehend von derselben Dehnung $\varepsilon_{e \max I}$ an der 


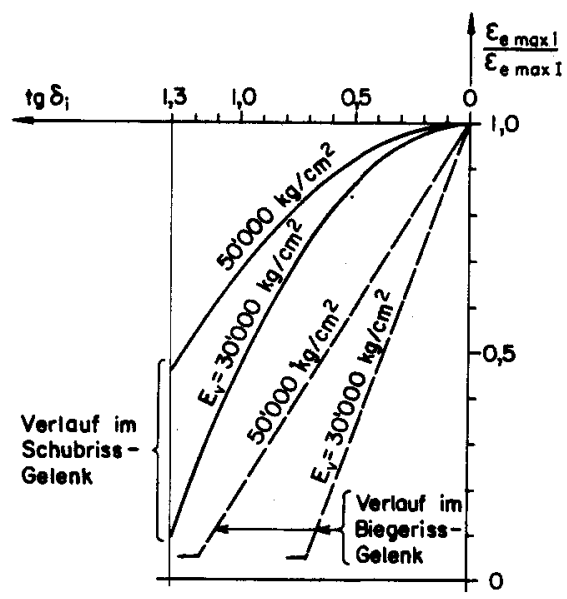

Bild 38

Verlauf der Dehnungen der Zugarmierung in den Rissequerschnitten (Einfluss des Verbundes auf den Verlauf zwischen den Rissen nicht bericksichtigt, Zahlenwerte siehe 3.42 )

meistbeanspruchten Stelle. Es wurden die folgenden Zahlenwerte zugrundegelegt:

$$
\begin{aligned}
& \frac{Z_{I}}{F_{e}}=5000 \mathrm{~kg} / \mathrm{cm}^{2}, \quad \frac{B_{45}}{Z_{I}}=0,20, \quad \varepsilon_{e \max I}=31 \% 0, \\
& E_{v}=30^{\prime} 000 \text { und } 50^{\prime} 000 \mathrm{~kg} / \mathrm{cm}^{2} .
\end{aligned}
$$

Die Darstellung zeigt wie erwartet, dass - ausgehend von der meistbeanspruchten Stelle - die Dehnungen in den Rissen umso weniger abnehmen, je grösser die Verfestigung ist.

Zum Vergleich wurde in Bild 38 auch der Verlauf der Dehnungen der Zugarmierung in den Rissen eines entsprechenden Biegeriss-Gelenkes eingetragen:

$$
\frac{\varepsilon_{\text {emaxi }}}{\varepsilon_{\text {emax } I}}=1-\frac{1}{\varepsilon_{e \max I}} \cdot \frac{Q}{B_{45}} \cdot \frac{B_{45}}{E_{v} F_{e}} \cdot \operatorname{tg} \delta_{i}
$$


$\mathrm{Zu}$ den obigen Zahlenwerten hinzu wurde noch $\mathrm{B}_{45} / \mathrm{Q}=0,8$ angenommen. Im Vergleich zum Schubriss-Gelenk zeigt sich eine bedeutend stärkere Dehnungsabnahme, was den Ergebnissen des vorangehenden Abschnittes 3.41 über den Zugkraftverlauf entspricht.

\subsection{Vergleich mit üblicher Betrachtungsweise}

Der gestrichelt eingetragene Verlauf der Zugkräfte beziehungsweise Dehnungen in den Bildern 37 und 38 ergibt sich auch unter der Annahme vom Ebenbleiben der Querschnitte. Die Linien stellen dann jedoch nicht nur die Werte in den Rissequerschnitten, sondern auch diejenigen zwischen den Rissen dar. Selbst wenn man diesen an sich wesentlichen Unterschied ausser acht lässt, zeigen die Darstellungen, dass die Hypothese vom Ebenbleiben der Querschnitte eine vollkommen untaugliche Grundlage ist, um die Kräfte und Formänderungen in einem auch durch Schub beanspruchten plastischen Gelenk zu erfassen. Denn im Bereich von Biegeschubrissen entstehen innere Kräfte und Verformungen, welche auch bei Mittelwertbildung auf der Grundlage der obigen Hypothese nicht erfasst werden können.

\subsection{Allgemeine Beurteilung}

\subsection{Verhalten des Schubriss-Gelenkes}

Im Abschnitt 3.3 wurde dargelegt, welche Ursachen zu einem Versagen im Schubriss-Gelenk führen können. Es wurde versucht, die Grundlagen für eine Berechnung der entsprechenden kritischen Gelenkwinkel zu entwickeln. Dabei ergab sich, dass diese Berechnung oft äusserst schwierig und aufwendig ist.

Im Abschnitt 3.4 wurde demgegenüber gezeigt, dass in einem Schubriss-Gelenk die Zugkraft in den Rissen und die entsprechenden Dehnungen von der meistbeanspruchten Stelle aus nur verhältnismässig wenig abnehmen. Bei üblichen Verhältnissen entstehen somit in der Zugarmierung über einen grösseren Bereich plastische Deformationen, das heisst, solche treten in mehreren Rissen auf. Diese Tatsache wurde in [7] und [8] experimentell nachgewiesen. Eine Kon- 
zentration der plastischen Deformationen auf den Bereich eines einzigen Risses, wie dies im Biegeriss-Gelenk vorkommen kann, tritt somit in einem SchubrissGelenk kaum auf. Plastische Deformationen in mehreren Rissen bedeutet jedoch zum vorneherein, dass der kritische Gelenkwinkel verhältnismässig gross ist. Vergleicht man diesen mit dem für eine Umlagerung der inneren Kräfte erforderlichen Winkel (siehe 4.), so gelangt man zu der folgenden Erkenntnis:

In Schubriss-Gelenken ist eine genauere Berechnung des kritischen Gelenkwinkels oft nicht nötig. Bei üblichen Verhältnissen genügt es meist, sich Rechenschaft zu geben, ob in mehreren Biegeschubrissen des Gelenkes plastische Deformationen der Zugarmierung auftreten.

Besteht jedoch über das Genügen der Deformationsfähigkeit des Gelenkes eine Unsicherheit, so kann, ausgehend von einem angenommenen Gelenkwinkel beziehungsweise einer Rissweite an der meistbeanspruchten Stelle, eine überschlägige Abschätzung des Zugkraft- beziehungsweise Dehnungsverlaufes vorgenommen werden, ohne dass ein Kriterium für das Versagen eingeführt wird. Hiezu sind die in den Abschnitten 3.21 und 3.31 entwickelten Grundlagen sowie das Spannungs-Dehnungs-Diagramm der Zugarmierung zu verwenden.

Diese Feststellungen gelten allerdings nur unter den folgenden Voraussetzungen:

- Keine extrem hohe Schubbeanspruchung (Schiefer Druck, Versagen des Betons im Steg),

- Genügende Bügelarmierung,

- Genügender Verbund der Längsarmierung.

Diese Voraussetzungen sind selbstverständlich stets zu überprüfen. Sind sie erfüllt, so fallen in vielen Fällen erhebliche Berechnungsschwierigkeiten dahin.

\subsection{Generelle Abhängigkeit des kritischen Gelenkwinkels in Biegeriss- und} Schubriss-Gelenken von der Schubbeanspruchung

In Bild 39 ist generell und nur qualitativ die Veränderung des kritischen Gelenkwinkels $\theta_{\mathrm{kr}}$ mit wachsender Schubbeanspruchung dargestellt. Nach ( 122 ) entstehen etwa für $\tau<\tau_{1}$ nur Biegerisse. Für $\tau>\tau_{1}$ treten Biegeschubrisse auf. Entsprechend entsteht ein Biegeriss- oder ein Schubriss-Gelenk. Im Biegeriss-Gelenk konzentrieren sich die plastischen Deformationen umso mehr auf 


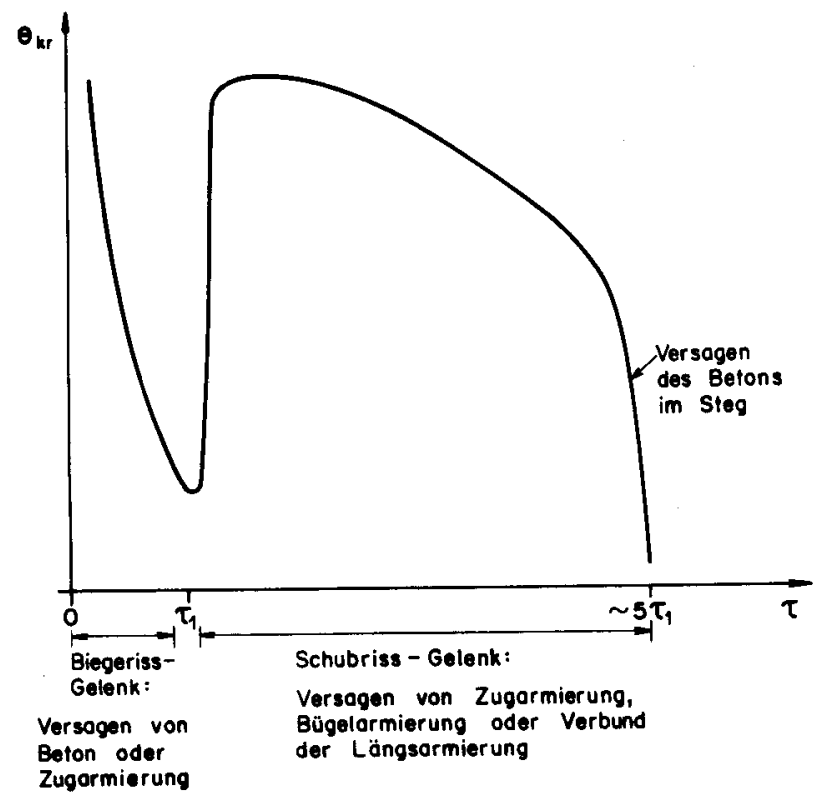

\section{Bild 39}

Generelle Abhängigkeit des kritischen Gelenkwinkels in Biegeriss- und Schubriss-Gelenken von der Schubbeanspruchung

einen kleinen Bereich, je grösser $\mathcal{T}$ ist. Demgemäss verringert sich der kritische Gelenkwinkel beim Versagen der Zugarmierung oder des Betons. Genügt jedoch die Schubbeanspruchung, um Biegeschubrisse zu erzeugen, so erstrecken sich die plastischen Deformationen über einen bedeutend grösseren Bereich. Trotz stärkerer Veränderung des äusseren Momentes entstehen in mehr Rissen plastische Deformationen, womit der Gelenkwinkel bis zum Eintreten einer der verschiedenen Versagensfälle ganz beträchtlich anwachsen kann.

Mit weiter zunehmender Schubbeanspruchung verringert sich dann auch im Schubriss-Gelenk der Bereich der plastischen Deformationen wieder, das heisst $\Theta_{\mathrm{kr}}$ nimmt - nur generell und qualitativ betrachtet - ab. Im speziellen kann 
natürlich $\Theta_{\mathrm{kr}}$ gering sein oder ganz verschwinden, insbesondere bei gănzlichem Ungenügen der Bügelarmierung oder des Verbundes der Längsarmierung. Genügen diese jedoch und tritt der in Schubriss-Gelenken äusserst seltene Fall eines Versagens der Zugarmierung nicht ein, so ergibt sich erst beim Auftreten hoher schiefer Druckspannungen eine drastische Reduktion des kritischen Gelenkwinkels. 


\section{ERFORDERLICHE GELENKWINKEL}

Die beiden vorangehenden Kapitel hatten im wesentlichen die Berechnung der sogenannten kritischen Gelenkwinkel, das heisst, der durch die Deformationsfähigkeit bedingten, maximal möglichen Rotationswinkel, zum Gegenstand. Demgegenuiber soll hier vor allem die Ermittlung derjenigen Gelenkwinkel behandelt werden, welche für das Erreichen der Traglast, also für eine vollstăndige Momentenumlagerung, erforderlich sind. Dabei wird von den Begriffen und Voraussetzungen der einfachen Plastizitätstheorie ausgegangen (siehe zum Beispiel

[1] , [2], [3] ). Wo es nötig und zweckmässig ist, werden später Modifikationen und Erweiterungen vorgenommen.

\subsection{Mechanismus und Traglast}

Die einfache Plastizitätstheorie setzt voraus, dass an der Stelle eines plastischen Gelenkes sich die Grösse des Momentes nicht mehr verändere, sobald der Wert des sogenannten plastischen Momentes (vergleiche 4. 32) erreicht ist. Hingegen soll die dortige Krümmung beziehungsweise der Rotationswinkel - für welche eine Konzentration auf einen einzigen Stabquerschnitt angenommen wird solange zunehmen können, bis genügend plastische Gelenke entstanden sind, damit sich im Tragwerk ein sogenannter Mechanismus ausbildet. Die zum Mechanismus gehörende Last wird als Traglast bezeichnet. Sie ist für die betreffende Lastanordnung die Höchstlast, welche das Tragwerk aufnehmen kann.

Die Verfahren zur Berechnung von Mechanismus und Traglast sind in der bereits angefuihrten einschlägigen Literatur dargestellt. Die Kenntnis dieser Verfahren wird im folgenden vorausgesetzt. Es sei hier lediglich darauf hingewiesen, dass dafür die beiden sogenannten Grenzwertsätze der Plastizitätstheorie die wichtigste theoretische Grundlage darstellen (vergleiche auch [4], [5] ).

\section{2. Berechnung der Gelenkwinkel}

\subsection{Zustand beim Erreichen der Traglast}

Die teilweise bereits erwähnten wichtigsten Voraussetzungen der einfachen Plastizitätstheorie können wie folgt zusammengefasst werden: 
a) In den plastischen Gelenken wirken während einer Rotation die konstanten plastischen Momente, welche bekannt sind.

b) Die plastischen Gelenke weisen eine Rotationsfähigkeit auf, welche zum Erreichen der Traglast genügt.

c) Die plastischen Deformationen in einem plastischen Gelenk konzentrieren sich auf einen einzigen Stabquerschnitt.

d) Es tritt kein Wandern von plastischen Gelenken auf.

e) Die Biege- und Schubsteifigkeiten der Stäbe zwischen den Gelenken sind bekannt.

f) Alle Lasten werden erstmals und proportional erhöht zwischen der Bildung des ersten plastischen Gelenkes und der Höchstlast (keine Entlastungen, Lastzyklen und so weiter, keine Wanderlasten).

g) Sämtliche Gleichgewichtsgleichungen dürfen am unverformten System formuliert werden.

Im weiteren setzen wir voraus:

h) Der richtige Mechanismus ist bekannt.

i) Die Reihenfolge, in welcher sich die plastischen Gelenke ausbilden, ist bekannt.

k) Die Traglast ist bekannt.

Die folgenden Ausführungen beschränken sich entsprechend dem Ziel dieser Arbeit auf statisch unbestimmte Durchlaufträger. Deren Gültigkeit könnte jedoch ohne besondere Schwierigkeiten auch auf andere Stabtragwerke erweitert werden.

Weist ein Durchlaufträger nur 2 Felder auf, so ist vor dem Ausbilden des letzten (das heisst in diesem Falle: des zweiten) plastischen Gelenkes das System statisch bestimmt geworden. Der nach Erreichen der Traglast entstehende (totale) Mechanismus umfasst das ganze Tragwerk. Sind jedoch drei oder mehr Felder vorhanden, so entsteht oft ein sogenannter lokaler Mechanismus. Vor dem Auftreten des letzten plastischen Gelenkes ist das Tragwerk immer noch statisch unbestimmt. 
Mit den Bezeichnungen

ñ: Grad der statischen Unbestimmtheit des noch unbelasteten Tragwerks, $\overline{\mathrm{m}}$ : Anzahl der plastischen Gelenke nach der Ausbildung eines Mechanismus, gilt je nach Ort eines allfälligen lokalen Mechanismus folgender Zusammenhang:

Innenfeld:

Aussenfeld:

Kragarm:

$$
\begin{aligned}
& 3 \leq \overline{\mathrm{m}} \leq \overline{\mathrm{n}}+1 \\
& 2 \leq \overline{\mathrm{m}} \leq \overline{\mathrm{n}}+1 \\
& 1 \leq \overline{\mathrm{m}} \leq \overline{\mathrm{n}}+1
\end{aligned}
$$

Die weiteren Ueberlegungen werden vorgenommen an dem möglichst allgemein gewählten Beispiel eines über sechs Felder durchlaufenden Trägers, bei welchem sich in einem Innenfeld ein lokaler Mechanismus ausbildet. In Bild 40 a) und b) sind das Tragwerk mit der Belastung sowie die schliesslich entstehenden plastischen Gelenke dargestellt. Die Reihenfolge, wie diese sich bilden, ist eingetragen.

a) Tragwerk und Belostung:

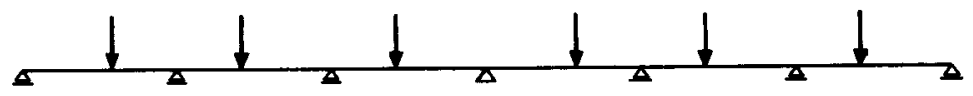

b) Plastische Gelenke (Mechanismus):

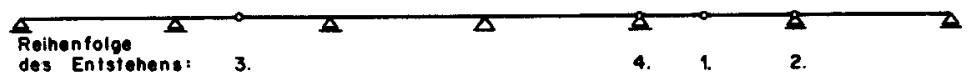

c) Grundsystem ("elastische" und plastische Gelenke und Momente):

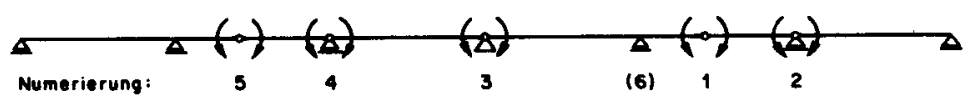

Bild 40

Beispiel eines Durchlaufträgers mit lokalem Mechanismus 
Betrachtet man den Zustand unmittelbar beim Erreichen der Traglast, das heisst vor der Ausbildung des letzten plastischen Gelenkes, so hat am Ort dieses Gelenkes das Moment wohl den Wert $M_{p}$ erreicht, doch ist die Gelenkrotation noch Null. In den übrigen plastischen Gelenken sind dann gerade die für das Erreichen der Traglast erforderlichen Rotationswinkel vorhanden. Um diese nach den üblichen Methoden der Elastizitätstheorie berechnen zu können, müssen im Falle eines lokalen Mechanismus zu den plastischen Gelenken hinzu noch soviele "elastische" Gelenke eingeführt werden, dass ein statisch bestimmtes Grundsystem entsteht. Für unser Beispiel ist das gewählte Grundsystem in Bild $40 \mathrm{c}$ ) dargestellt. Zweckmässigerweise werden vorerst die Gelenke des lokalen Mechanismus in der Reihenfolge ihres Entstehens numeriert, daran schliessen die übrigen elastischen und plastischen Gelenke an.

Für das Grundsystem des Beispiels kann folgende allgemeine Matrix aufgestellt werden:

\begin{tabular}{l|llllll} 
& $M_{1}$ & $M_{2}$ & $M_{3}$ & $M_{4}$ & $M_{5}$ & $P$ \\
\hline$\beta_{1}$ & $\alpha_{11}$ & $\alpha_{12}$ & $\alpha_{13}$ & $\alpha_{14}$ & $\alpha_{15}$ & $\alpha_{10}$ \\
$\beta_{2}$ & $\alpha_{21}$ & $\alpha_{22}$ & $\alpha_{23}$ & $\alpha_{24}$ & $\alpha_{25}$ & $\alpha_{20}$ \\
$\beta_{3}$ & $\alpha_{31}$ & $\alpha_{32}$ & $\alpha_{33}$ & $\alpha_{34}$ & $\alpha_{35}$ & $\alpha_{30}$ \\
$\beta_{4}$ & $\alpha_{41}$ & $\alpha_{42}$ & $\alpha_{43}$ & $\alpha_{44}$ & $\alpha_{45}$ & $\alpha_{40}$ \\
$\beta_{5}$ & $\alpha_{51}$ & $\alpha_{52}$ & $\alpha_{53}$ & $\alpha_{54}$ & $\alpha_{55}$ & $\alpha_{50}$
\end{tabular}

Darin bedeuten:

$\beta \quad$ Drehwinkel in einem (elastischen oder plastischen) Gelenk des statisch bestimmten Grundsystems,

M Moment in einem (elastischen oder plastischen) Gelenk des statisch bestimmten Grundsystems, 

$\mathbf{P}$
Lastintensität,
$\alpha$
Matrix-Koeffizient.

In allen jenen Gelenken, wo plastische Rotationen auftreten, ist der Drehwinkel unbekannt, hingegen ist das Moment gleich dem plastischen Moment. In den übrigen (elastischen) Gelenken jedoch ist das Moment unbekannt, und der Drehwinkel muss gleich Null sein. Die Variablen der Matrix ( 175 ) können daher mit den weiteren Grössen

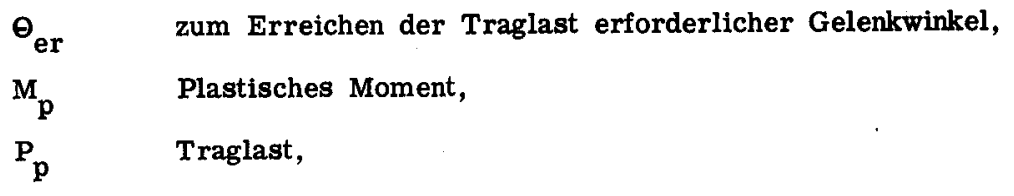

wie folgt noch näher bezeichnet werden:

\begin{tabular}{l|cccccc} 
& $M_{1}$ & $M_{2}$ & $M_{3}$ & $M_{4}$ & $M_{5}$ & $P$ \\
$\left(=M_{p 1}\right)$ & $\left(=M_{p 2}\right)$ & & & $\left.M_{p 5}\right)$ & $\left(=P_{p}\right)$ \\
\hline$\beta_{1}\left(=\theta_{\text {er } 1}\right)$ & $\alpha_{11}$ & $\alpha_{12}$ & $\alpha_{13}$ & $\alpha_{14}$ & $\alpha_{15}$ & $\alpha_{10}$ \\
$\beta_{2}\left(=\theta_{\text {er 2 }}\right)$ & $\cdot$ & $\cdot$ & $\cdot$ & $\cdot$ & $\cdot$ & $\cdot$ \\
$\beta_{3}(=0)$ & $\cdot$ & $\cdot$ & $\cdot$ & $\cdot$ & $\cdot$ & $\cdot$ \\
$\beta_{4}(=0)$ & $\cdot$ & $\cdot$ & $\cdot$ & $\cdot$ & $\cdot$ & $\cdot$ \\
$\beta_{5}\left(=\theta_{\text {er 5 }}\right)$ & $\alpha_{51}$ & $\alpha_{52}$ & $\alpha_{53}$ & $\alpha_{54}$ & $\alpha_{55}$ & $\alpha_{50}$
\end{tabular}

Die gesuchten Winkel $\theta_{\text {er }}$ lassen sich aus ( 176 ) auf sehr einfache Weise durch das Verfahren der sogenannten Austausch-Schritte (siehe zum Beispiel [22]) ermitteln. Nach dem Austausch von $\beta_{3}$ mit $M_{3}$ (Pivotelement $\alpha_{33}$ ) und von $\beta_{4}$ 
mit $M_{4}$ (Pivotelement $\alpha_{44}$ ) hat die Matrix folgendes Aussehen:

\begin{tabular}{l|cccccc} 
& $M_{1}$ & $M_{2}$ & $\beta_{3}$ & $\beta_{4}$ & $M_{5}$ & $P$ \\
\hline$\beta_{1}\left(=\theta_{\text {er 1 }}\right)$ & $\alpha_{11}^{\prime}$ & $\alpha_{12}^{\prime}$ & $\alpha_{13}^{\prime}$ & $\alpha_{14}^{\prime}$ & $\alpha_{15}^{\prime}$ & $\alpha_{10}^{\prime}$ \\
$\beta_{2}\left(=\theta_{\text {er 2 }}\right)$ & $\cdot$ & $\cdot$ & $\cdot$ & $\cdot$ & $\cdot$ & $\cdot$ \\
$M_{3}$ & $\cdot$ & $\cdot$ & $\cdot$ & $\cdot$ & $\cdot$ & $\cdot$ \\
$M_{4}$ & $\cdot$ & $\cdot$ & $\cdot$ & $\cdot$ & $\cdot$ & $\cdot$ \\
$\beta_{5}\left(=\theta_{\text {er 5 }}\right)$ & $\alpha_{51}^{\prime}$ & $\alpha_{52}^{\prime}$ & $\alpha_{53}^{\prime}$ & $\alpha_{54}^{\prime}$ & $\alpha_{55}^{\prime}$ & $\alpha_{50}^{\prime}$
\end{tabular}

Nach dieser teilweisen Inversion sind die Matrix-Koeffizienten zur Unterscheidung von jenen der Matrix ( 176 ) mit einem ' bezeichnet.

In ( 177 ) stehen nun sämtliche bekannten Variablen am oberen Rand und alle unbekannten Variablen am linken Rand der Matrix. Es können also - auch im Falle eines lokalen Mechanismus - die zum Erreichen der Traglast erforderlichen Gelenkwinkel stets als Linearformen der plastischen Momente in allen plastischen Gelenken des Tragwerks (mit Ausnahme des letzten) und der Traglast dargestellt werden. Dieses einfache Ergebnis ist besonders wichtig im Hinblick auf die Möglichkeit, in den plastischen Gelenken modifizierte plastische Momente zu berücksichtigen, die grösser als die plastischen Momente sind (vergleiche 4.3). Der Matrix ( 177 ) können ferner auch die Momente in den elastischen Gelenken $\left(M_{3}, M_{4}\right)$ entnommen werden. Sie kommen jedoch in den Bestimmungsgleichungen für $\Theta_{\text {er }}$ nicht vor.

Zur Wahl der elastischen Gelenke ist noch beizufügen, dass diese vorteilhafterweise über $Z$ wischenstützen angenommen werden, genau wie bei der elastizitätstheoretischen Berechnung von Durchlaufträgern. Befinden sich auch alle plasti- 
schen Gelenke, mit Ausnahme des letzten, über Zwischenstiutzen, so entsprechen die Koeffizienten der Matrix ( 175 ) denjenigen der bekannten 3-Momentengleichungen des Durchlaufträgers.

\subsection{Zustände vor dem Erreichen der Traglast}

\section{a) Allgemein}

Es kann erwünscht oder notwendig sein, nicht nur die für das Erreichen der Traglast erforderlichen Gelenkwinkel, sondern für irgend einen vorangehenden Zustand die folgenden Daten zu kennen:

- Bisherige Rotationswinkel

- Zugehörige Last

- Entsprechende Momente in allen Gelenken

Diese Daten werden beispielsweise gesucht für den Zustand, bei welchem in einem andern als dem letzten Gelenk gerade das plastische Moment auftritt, also der Gelenkwinkel gerade noch Null ist. Oder es interessiert der Fall, da in einem bestimmten plastischen Gelenk ein bestimmter Gelenkwinkel (zum Beispiel $\theta_{\mathbf{k r}}$ für $\theta_{\mathbf{k r}}<\Theta_{\mathrm{er}}$ ) vorhanden ist. Jedenfalls muss der Zustand stets durch die gegebene Grösse einer bestimmten Variablen fixiert sein. Zum Beispiel werden beim Durchlaufträger Bild 40 die obigen Daten gesucht für den Augenblick, da an der Stelle des Gelenkes Nr. 2 gerade das plastische Moment erreicht wird. Dann sind im Grundsystem in den plastischen Gelenken Nr. 2 und 5 sowie ohnehin in den elastischen Gelenken Nr. 3 und 4 die Gelenkwinkel Null. Einzig im Gelenk Nr. 1 ist ein bestimmter Rotationswinkel vorhanden. Sollen in der Matrix wieder die bekannten Variablen oben stehen, so müssen in ( 175 ) vorerst vier Austausch-Schritte mit den Diagonalelementen der 2., 3., 4. und 5. Zeile als Pivotelemente vorgenommen sowie anschliessend noch $\mathbf{P}$ mit dem bekannten Moment $\mathrm{M}_{2}$ ausgetauscht werden: 


\begin{tabular}{l|cccccc} 
& $M_{1}$ & $\beta_{2}$ & $\beta_{3}$ & $\beta_{4}$ & $\beta_{5}$ & $M_{2}$ \\
& $\left(=M_{p 1}\right)$ & $(=0)$ & $(=0)$ & $(=0)$ & $(=0)$ & $\left(=M_{p 2}\right)$ \\
\hline$\beta_{1}\left(=\theta_{1}\right)$ & $\alpha_{11}^{\prime \prime}$ & $\alpha_{12}^{\prime \prime}$ & $\alpha_{13}^{\prime \prime}$ & $\alpha_{14}^{\prime \prime}$ & $\alpha_{15}^{\prime \prime}$ & $\alpha_{10}^{\prime \prime}$ \\
\hline$M_{3}\left(=P_{2}\right)$ & $\cdot$ & $\cdot$ & $\cdot$ & $\cdot$ & $\cdot$ & $\cdot$ \\
$M_{4}$ & $\cdot$ & $\cdot$ & $\cdot$ & $\cdot$ & $\cdot$ & $\cdot$ \\
$M_{5}$ & $\cdot$ & $\cdot$ & $\cdot$ & $\cdot$ & $\cdot$ & $\cdot$ \\
& $\alpha_{51}^{\prime \prime}$ & $\alpha_{52}^{\prime \prime}$ & $\alpha_{53}^{\prime \prime}$ & $\alpha_{54}^{\prime \prime}$ & $\alpha_{55}^{\prime \prime}$ & $\alpha_{50}^{\prime \prime}$
\end{tabular}

Damit sind für den oben fixierten Zustand die gesuchten Grössen als einfache Linearformen der bekannten plastischen Momente in den Gelenken Nr. 1 und 2 ausgedrückt.

Dieses soeben skizzierte Verfahren kann noch allgemeiner dargestellt werden. Es bedeuten:

$$
\begin{array}{ll}
\overline{\mathrm{a}}: & \begin{array}{l}
\text { Vektor der abhängigen Variablen } \\
\text { (Randspalte links) }
\end{array} \\
\overline{\mathrm{b}}: \quad \begin{array}{l}
\text { Vektor der unabhängigen Variablen } \\
\text { (Kopfzeile) }
\end{array}
\end{array}
$$

Diese Vektoren sind über die entsprechende Matrix wie folgt verknüpft:

$$
\overline{\mathbf{a}}=[\mathrm{M}] \overline{\mathbf{b}}
$$

Grundlage ist stets die Elastizitätsmatrix ( 175 ). Für die daraus durch Austauschschritte zu ermittelnden Matrizen werden im folgenden jeweils nur die transponierten Variablen-Vektoren angeschrieben.

Die verschiedenen Tragsysteme beziehungsweise Matrizen, die sich entsprechend der Ausbildung der plastischen Gelenke ergeben, können zum Beispiel nach zu- 
nehmender Lastintensität geordnet werden:

$\mathbf{P}<\mathrm{P}_{1}$

$$
\begin{aligned}
& \overline{\mathbf{a}}^{\prime}=\left(\mathrm{M}_{1}, \mathrm{M}_{2}, \mathrm{M}_{3}, \mathrm{M}_{4}, \mathrm{M}_{5}\right) \\
& \overline{\mathrm{b}}^{\prime}=\left(\beta_{1}=0, \beta_{2}=0, \beta_{3}=0, \beta_{4}=0, \beta_{5}=0, \mathrm{P}<\mathrm{P}_{1}\right)
\end{aligned}
$$

$P_{1}$ wird gelegentlich auch Fliesslast genannt. Ihr entspricht die Matrix mit den folgenden Variablen-Vektoren:

$\mathbf{P}=\mathbf{P}_{1}$

$$
\begin{aligned}
& \bar{a}^{\prime}=\left(P=P_{1}, M_{2}, M_{3}, M_{4}, M_{5}\right) \\
& \bar{b}^{\prime}=\left(\beta_{1}=0, \beta_{2}=0, \beta_{3}=0, \beta_{4}=0, \beta_{5}=0, M_{1}=M_{p 1}\right)
\end{aligned}
$$

Mit weiter wachsender Lastintensität ergeben sich die Variablen-Vektoren der entsprechenden Matrizen wie folgt:

$$
\begin{aligned}
P_{1}<P<P_{2}: & \\
\bar{a}^{\prime} & =\left(\beta_{1}=\theta_{1}, M_{2}, M_{3}, M_{4}, M_{5}\right) \\
\bar{b}^{\prime} & =\left(M_{1}=M_{p 1}, \beta_{2}=0, \beta_{3}=0, \beta_{4}=0, \beta_{5}=0, P_{1}<P<P_{2}\right)
\end{aligned}
$$

$\mathbf{P}=\mathbf{P}_{\mathbf{2}}$ :

$$
\begin{aligned}
& \overline{\mathbf{a}}^{\prime}=\left(\beta_{1}=\Theta_{1}, \quad P=P_{2}, M_{3}, M_{4}, M_{5}\right) \\
& \bar{b}^{\prime}=\left(M_{1}=M_{p 1}, \beta_{2}=0, \beta_{3}=0, \beta_{4}=0, \beta_{5}=0, M_{2}=M_{p 2}\right)
\end{aligned}
$$

$P_{2}<P<P_{5}:$

$$
\begin{aligned}
& \overline{\mathrm{a}}^{\prime}=\left(\beta_{1}=\Theta_{1}, \beta_{2}=\Theta_{2}, M_{3}, M_{4}, M_{5}\right) \\
& \bar{b}^{\prime}=\left(M_{1}=M_{p 1}, M_{2}=M_{p 2}, \beta_{3}=0, \beta_{4}=0, \beta_{5}=0, P_{2}<P<P_{5}\right)
\end{aligned}
$$

$\mathbf{P}=\mathbf{P}_{5}$;

$$
\begin{aligned}
& \overline{\mathrm{a}}^{\prime}=\left(\beta_{1}=\theta_{1}, \beta_{2}=\theta_{2}, M_{3}, M_{4}, \quad P=P_{5}\right) \\
& \overline{\mathrm{b}}^{\prime}=\left(\mathrm{M}_{1}=\mathrm{M}_{\mathrm{p} 1}, \mathrm{M}_{2}=\mathrm{M}_{\mathrm{p} 2}, \beta_{3}=0, \beta_{4}=0, \beta_{5}=0, M_{5}=M_{\mathrm{p} 5}\right)
\end{aligned}
$$




$$
\begin{aligned}
P_{5}<P<P_{p}: & \\
\bar{a}^{\prime} & =\left(\beta_{1}=\Theta_{1}, \beta_{2}=\Theta_{2}, M_{3}, M_{4}, \beta_{5}=\Theta_{5}\right) \\
\bar{b}^{\prime} & =\left(M_{1}=M_{p 1}, M_{2}=M_{p 2}, \beta_{3}=0, \beta_{4}=0, M_{5}=M_{p 5}, P_{5}<P<P_{p}\right)
\end{aligned}
$$

Wird in ( 184 ) die Last $P$ gleich der Traglast $P_{p}$ gesetzt, so ergibt sich die Matrix ( 177 ) mit den für das Erreichen der Traglast erforderlichen Gelenkwinkeln.

Das Verfahren kann allgemein wie folgt kurz zusammengefasst werden:

Ausgehend von der Elastizitäts-Matrix des statisch bestimmten Grundsystems lassen sich durch Austausch-Schritte die Zusammenhänge zwischen den Variablen

- Rotationswinkel in den plastischen Gelenken,

- Lastintensität,

- Momente an den Stellen der elastischen und der (eventuell teilweise noch nicht aufgetretenen) plastischen Gelenke.

auf einfache Weise darstellen, und zwar für jeden Zustand, dem eine Last entspricht, die nicht höher als die Traglast ist.

\section{b) Zusammenhang zwischen Gelenkwinkel und Querkraft}

Im Abschnitt 3.3 ergab sich, dass in einem Schubriss-Gelenk für die Ermittlung des kritischen Gelenkwinkels infolge einiger Versagensursachen der Zusammenhang $z$ wischen Gelenkwinkel und Querkraft, also die Funktion $\Theta(Q)$, bekannt sein sollte (vergleiche Bild 35). Mit dem vorstehend entwickelten Verfahren ist es möglich, diese Funktion punktweise zu berechnen.

Es wurde gezeigt, dass sämtlichen Tragsystemen, welche bei steigender Lastintensität über die Fliesslast bis zur Traglast entstehen, ganz bestimmte Matrizen entsprechen. Eine Matrix gilt zum Beispiel für sämtliche Zustände zwischen der Bildung zweier aufeinanderfolgender Gelenke. Anstatt durch die Lastintensität 
kann ein Zustand auch durch ein bestimmtes Moment oder einen bestimmten Rotationswinkel in einem plastischen Gelenk charakterisiert werden. Man kann also natürlich nur in den für ein bestimmtes Tragsystem geltenden Grenzen - zum Beispiel das Moment in einem sich demnächst bildenden plastischen Gelenk vorschreiben und aus der entsprechenden Matrix - über die Lastintensität und die Momente in den übrigen elastischen und plastischen Gelenken - die zugehörige Querkraft sowie den Rotationswinkel berechnen.

Beispielsweise sei für das Tragwerk Bild 40 die Funktion $\Theta(Q)$ im Gelenk Nr. 1 zu ermitteln. Mit der Annahme, dass die Querkraft zwischen den Gelenken Nr. 1 und 2 interessiert, kann die gesuchte Funktion mit $\Theta_{1}\left(Q_{1,2}\right)$ bezeichnet werden. Dem Nullpunkt dieser Funktion entspricht der Zustand, da im Gelenk Nr. 1 das plastische Moment wohl gerade erreicht, der Rotationswinkel jedoch noch Null ist. Es gilt somit die Matrix mit den Variablen-Vektoren ( 180 ). Zustände, welche nach dem Rotationsbeginn im Gelenk Nr. 2 auftreten, können durch die Grösse des Momentes $M_{2}$ fixiert werden. Wird das Moment im Gelenk Nr. 2 bei Rotationsbeginn im Gelenk Nr. 1 mit $\mathrm{M}_{2,1}$ bezeichnet, so gilt für den Variationsbereich von $M_{2}$ :

$$
\mathrm{M}_{2,1}<\mathrm{M}_{2} \leq \mathrm{M}_{\mathrm{p} 2}
$$

Das Tragsystem entspricht demjenigen der Matrix mit den Vektoren ( 181 ). Da jedoch anstelle der Lastintensität $P$ das Moment $M_{2}$ variiert wird, ist die Matrix ( 178 ) mit $\mathrm{M}_{2,1}<\mathrm{M}_{2} \leq \mathrm{M}_{\mathrm{p} 2}$ zu verwenden.

Für Zustănde nach dem Rotationsbeginn im Gelenk $\mathrm{Nr} .2$ ist analog zu verfahren. Es ergibt sich jeweils der Rotationswinkel $\theta_{1}$, die zugehörige Last und die Momentenfläche, woraus $Q_{1,2}$ berechnet werden kann. Sind alle (in unserem Beispiel: 4) Tragsysteme durchlaufen, so ist die gesuchte Funktion $\Theta_{1}\left(Q_{1,2}\right)$ im ganzen Bereich vom Rotationsbeginn im Gelenk Nr. 1 bis zum Erreichen der Traglast punktweise bestimmt worden. 


\section{3 Besonderheiten bei Stahlbetonbalken}

Während die bisherigen Ausführungen dieses Kapitels grundsätzlich auf den eingangs zitierten Vorausset zungen der einfachen Plastizitätstheorie basierten, ist in diesem Abschnitt der Zusammenhang mit dem komplexen Verbundbaustoff Stahlbeton herzustellen.

\subsection{Rotationsbeginn}

In einem Stahlbetonbalken soll dann ein plastisches Gelenk entstehen, wenn in einem Querschnitt die nominelle Stahldehnung in der Zugarmierung nach Bild 2 erreicht beziehungsweise gerade überschritten wird (vergleiche 2.11 und 3.11):

$$
\varepsilon_{\mathrm{emax}} \geq \varepsilon_{\mathrm{f}}
$$

Verformt sich die Zugarmierung über $\varepsilon_{f}$ hinaus, so kann im Sinne der Plastizitätstheorie von einer "plastischen Rotation" gesprochen werden.

Zwischen dieser idealisierten Definition für die Ausbildung eines plastischen Gelenkes, beziehungsweise dem Rotationsbeginn, und der Wirklichkeit ergibt sich dann ein Unterschied, wenn die Zugarmierung aus kaltverformtem Stahl besteht (vergleiche Bild 2). Im Anfangsbereich der plastischen Verformungen ( $\varepsilon_{\text {plast }} \leq$ $2 \% 0$ ) zeigen sich gewisse Abweichungen, die jedoch für das Ergebnis einer plastizitätstheoretischen Berechnung nicht wesentlich sind, da sie nur unbedeutende Fehler bewirken. Sie können daher vernachlässigt werden.

\subsection{Momente in den plastischen Gelenken}

\section{a) Plastische Momente}

Das plastische Moment $M_{p}$ eines auf Biegung beanspruchten Stahlbetonquerschnittes definieren wir wie folgt: 


$$
\begin{array}{r}
-149- \\
M_{p}=G_{f} \cdot F_{e} \cdot y
\end{array}
$$

Darin bedeuten:

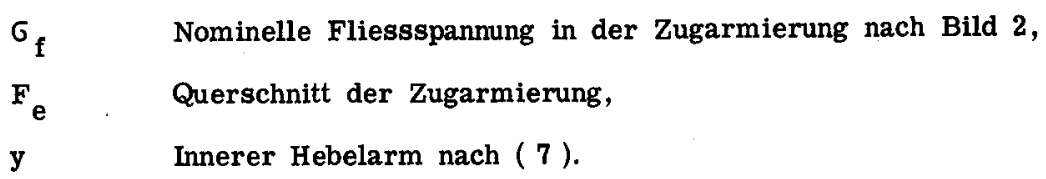

In erster Näherung ist somit auch bei Stahlbetonbalken ein plastisches Moment im Sinne der Plastizitătstheorie vorhanden. Dieses wird vorerst zum Beispiel für eine erste Traglastberechnung als konstant, das heisst als unabhängig vom Rotationswinkel, angenommen. Bei grösseren plastischen Verformungen kann jedoch die Abweichung des tatsächlichen Spannungs-Dehnungs-Diagramms der Zugarmierung vom ideal-plastischen Verlauf wesentlich werden, sodass wir noch ein sogenanntes modifiziertes plastisches Moment definieren müssen.

\section{b) Modifizierte plastische Momente}

Aufgrund der Untersuchungen im 2. Kapitel wissen wir, dass sowohl in einem Querschnitt mit kaltverformter wie auch in einem solchen mit naturharter Zugarmierung sofort nach Erreichen von $\sigma_{f}$ die Verfestigung beginnt. Welche Stahlbeanspruchung bei einem bestimmten Gelenkwinkel vorhanden ist, hängt bekanntlich von vielerlei Parametern, worunter auch die Eigenschaften der beteiligten Materialien, ab und kann nicht zum vorneherein angenommen werden. Allgemein treten im meistbeanspruchten Querschnitt Spannungen $\sigma_{\mathrm{f}} \leq \sigma_{\mathrm{e} \max } \leq \beta_{\mathrm{z}}$ und Dehnungen $\varepsilon_{f} \leq \varepsilon_{\text {emax }} \leq \lambda_{\mathrm{Gl}}$ auf. Werden der Berechnung der für das Erreichen der Traglast erforderlichen Gelenkwinkel - oder auch anderer Grössen stets nur die plastischen Momente zugrundegelegt, so können unter Umständen erhebliche Fehler entstehen. In manchen Fällen werden deshalb vorteilhafterweise "modifizierte plastische Momente" $M_{p}^{\prime}$ verwendet:

$$
\mathbf{M}_{\mathbf{p}}^{\prime}=\sigma_{\mathrm{emax}} \cdot \mathbf{F}_{\mathbf{e}} \cdot \mathrm{y}
$$


$\sigma_{\text {emax }}$ bedeutet also die im meist beanspruchten Querschnitt des plastischen Gelenkes vorhandene Stahlspannung. Hie und da genügt es, sie zu schätzen. Meist wird man aber $\sigma_{\text {e max }}$ durch eine wenigstens näherungsweise Untersuchung der Spannungs - und Dehnungsverteilung im betreffenden Gelenk ermitteln. Ist $\sigma_{\mathrm{emax}}=$ $\beta_{z}$, so geht $M_{p}^{\prime}$ nach ( 186 ) über in $M_{k r e}$ nach ( 149 ).

\subsection{Steifigkeiten}

Im Abschnitt 4.2 war vorausgesetzt worden, dass die Matrix-Koeffizienten $\alpha_{i k}$ bekannt beziehungsweise berechenbar seien. Bei Biegeträgern werden sie aufgrund der Arbeitsgleichung allgemein wie folgt ermittelt:

$$
\alpha_{i k}=\int \frac{M M^{\prime}}{E I} d x+\int \frac{Q Q^{\prime}}{G F^{\prime}} d x
$$

Die Grössen $M^{\prime}$ und $Q^{\prime}$ entsprechen dem sogenannten virtuellen Belastungszustand (siehe zum Beispiel [23]). M/EI und Q/GF' stellen die Verformungen infolge des effektiven Verschiebungszustandes dar. Da diese in Stahlbetonquerschnitten im allgemeinen nicht proportional zur Beanspruchung anwachsen, muss hier noch das Problem der Biege- und Schubsteifigkeit behandelt werden.

\section{a) Biegesteifigkeit}

Vorerst gilt es zu unterscheiden zwischen Bereichen mit und solchen ohne Biegerissen. Ueberall dort, wo das Moment das Rissmoment

$$
\mathbf{M}_{\mathbf{R}}=\mathrm{W} \cdot \beta_{\mathrm{bz}}
$$

nicht erreicht, kann die Steifigkeit EI wie üblich angesetzt werden:

$$
\mathbf{E I}=\mathbf{E}_{\mathrm{b}} \mathbf{I}_{\mathrm{id}}
$$


$E_{b}$ bedeutet den $E$-Modul des Betons für kurzfristige Beanspruchungen. $I_{i d}$ ist das sogenannte ideelle Trägheitsmoment des ungerissenen Verbundquerschnittes, bei dessen Berechnung in bekannter Weise die Stahlquerschnitte mit dem $\mathrm{n}$-fachen Gewicht zu berücksichtigen sind. Dabei nimmt $n=E_{e} / E_{b}$ meist etwa einen Wert zwischen 5 und 7 an.

Angesichts der Unsicherheit bei der Definition des $\mathrm{E}_{\mathrm{b}}$-Moduls und dessen Streuungen rechtfertigt es sich allerdings oft nicht, das ideelle Trägheitsmoment $\mathrm{zu}$ berechnen, und es kann mit genügender Genauigkeit gesetzt werden:

$$
E I \cong E_{b} I
$$

I ist dann das Trägheitsmoment des als homogen betrachteten Betonquerschnittes. Ist das Moment jedoch grösser als das Rissmoment, so ist mit dem Auftreten von Rissen zu rechnen. Zur Bestimmung der Biegesteifigkeit im Rissezustand wird vorausgesetzt, dass die Zugarmierung bis zum Erreichen von $\sigma_{f}$ ausschliesslich elastische Verformungen erleidet (idealisiertes Spannungs-Dehnungs-Diagramm Bild 2). Betrachten wir ein einzelnes Biegeriss-Element nach Bild 4, das nun jedoch ausserhalb eines plastischen Gelenkes liegen soll, so ergibt sich analog zu ( 10 ) und ( 38 ) der Risswinkel zu

$$
\omega=\frac{\varepsilon_{\mathrm{e} \max } \cdot \kappa}{\mathrm{h}-\mathbf{z}} \cdot \mathbf{s}
$$

Da $\varepsilon_{\text {emax }} \leq \varepsilon_{f}$, wird daraus mit

$$
\sigma_{\text {emax }}=\frac{\varepsilon_{\text {e max }}}{\bar{E}_{e}}
$$

und ( 69 ):

$$
\omega=\frac{M}{F_{e} \cdot y \cdot E_{e}} \cdot \kappa \cdot \frac{s}{h-z}
$$


Andererseits gilt nach der herkömmlichen Schreibweise für ein längs des Elementes konstantes Biegemoment (Gleichmässige Verteilung der Verformungen):

$$
\omega=\frac{M}{E I} \cdot \mathbf{s}
$$

Somit wird mit $\mathrm{y}=\eta \cdot \mathrm{h}$ die gesuchte Biegesteifigkeit im Rissezustand:

$$
E I=\frac{F_{e} \cdot E_{e} \cdot \eta \cdot h \cdot(h-z)}{K}
$$

Der Verbundkoeffizient $\kappa$ nach ( 15 ) berücksichtigt die Abnahme der Stahldehnungen zwischen den Biegerissen, welche gewissermassen die Biegesteifigkeit erhöht. Um $\kappa$ abzuschätzen, nehmen wir năherungsweise eine konstante Verbundspannung

$$
\tau_{v}(v) \cong \text { konst. }=\tau_{v}^{*}
$$

zwischen Beton und Zugarmierung an. Praktische Werte für $\tau_{\mathbf{V}}^{*}$ können den entsprechenden Verbundgesetzen oder auch den Gleichungen ( 65$)$ mit $\tau_{v}^{*} \cong \tau_{v R}$ entnommen werden. Mit dieser Annahme, sowie wegen (191), wird der Dehnungsverlauf zwischen den Rissen durch Geraden dargestellt.

Für den sehr hăfigen Fall, dass

$$
\Delta \sigma_{\text {etot }}=\tau_{\mathrm{v}}^{*} \cdot \frac{4}{\mathrm{~d}} \cdot \frac{\mathrm{s}}{2} \leq \sigma_{\mathrm{emax}}=E_{\mathrm{e}} \cdot \varepsilon_{\mathrm{emax}},
$$

was $\kappa \geq 0,5$ entspricht, wird:

$$
\kappa=1-\frac{\Delta \sigma_{e \text { tot }}}{2 \cdot \sigma_{e \max }}=1-\left(\frac{s}{d}\right) \cdot\left(\frac{\tau_{v}^{*}}{\sigma_{e \max }}\right)
$$

Ist hingegen $\Delta \sigma_{\text {etot }}>G_{\text {emax }}$, respektive resultiert aus (195) $x \leq 0,5$, so gilt: 


$$
\kappa=0,5 \cdot \frac{\sigma_{e \max }}{\Delta \sigma_{e \text { tot }}}=\frac{0,25}{\left(\frac{s}{d}\right) \cdot\left(\frac{T_{Y}^{*}}{\sigma_{e \max }}\right)}
$$

In Bild 41 sind die Gleichungen ( 195 ) und ( 196 ) für die häufigsten Zahlenbereiche der Verhälnisse $s / d$ und $\tau_{v}^{*} / \sigma_{e \max }$ zient $k$ kann direkt daraus entnommen werden.

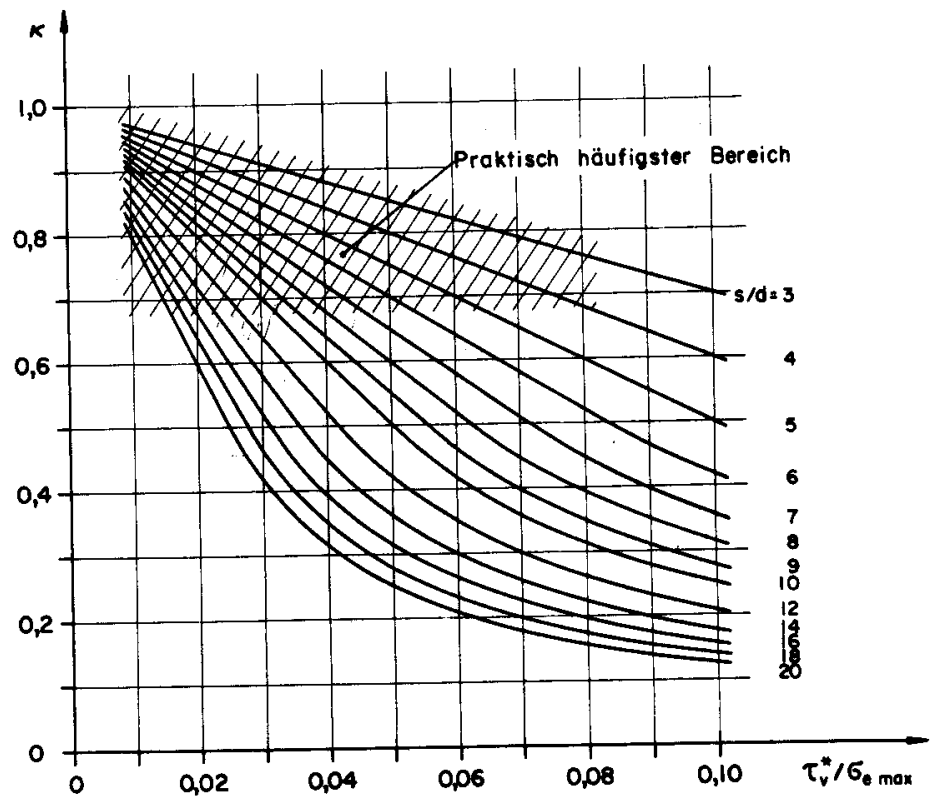

Bild 41

Verbundkoeffizient zur Bestimmung der Biegesteifigkeit im Bereich elastischer Verformungen der Zugarmierung 
Für die Bestimmung der Steifigkeit ist stets die Beanspruchung des betrachteten Zustandes massgebend, bei der Biegesteifigkeit also die Momentenlinie nach $\mathrm{Su}-$ perposition der Wirkungen sämtlicher am statisch bestimmten Grundsystem angreifenden Kräfte und Momente. Zum Beispiel für die Berechnung der zum Erreichen der Traglast erforderlichen Gelenkwinkel sollte die Momentenlinie unter der Traglast den Annahmen über den Verlauf der veränderlichen Biegesteifigkeit längs den verschiedenen Stäben zugrundegelegt werden. Dies ist insbesondere von Bedeutung für den Uebergang von ungerissenen zu gerissenen Balkenbereichen $\left(M=M_{R}\right)$, für die Stellen der Momentennullpunkte $(M=0)$, sowie für die Annahme von $G_{e \max }$ in der Bestimmungsgleichung für den Verbundkoeffizienten. Es ergibt sich hier die Schwierigkeit, dass der Verlauf dieser die Steifigkeit beeinflussenden Momente erst als Resultat der vorzunehmenden Berechnung erscheint. Dies ist besonders beim Auftreten von lokalen Mechanismen der Fall. Hier bleibt nichts anderes übrig, als von einem vorerst geschätzten Momentenverlauf auszugehen, die Steifigkeiten und Matrix-Koeffizienten entsprechend zu bestimmen und am Schluss der Berechnung die Annahmen zu ïberprüfen und eventuell zu verbessern. Eine solche Iteration ist natürlich sehr mühsam und aufwendig. Da jedoch eine gewisse Verschiebung der Stellen von $M=M_{R}$ und $M=0$ sowie eine beschränkte Veränderung von $G_{e \max }$ in den Ausdrücken für * auf das Resultat der Berechnung im allgemeinen keinen grossen Einfluss haben, werden indessen die ersten Annahmen meist genügend genau sein.

\section{b) Schubsteifigkeit}

Noch mehr als die Biegesteifigkeit ist bei Stahlbetonbalken die Schubsteifigkeit von der Beanspruchung abhängig. Solange keine schiefen Risse entstehen, ist die Schubsteifigkeit ausserordentlich gross. In diesem Fall ist das Glied in der Arbeitsgleichung ( 187 ), welches den Einfluss der Querkraft beruicksichtigt, vernachlässigbar klein.

Treten jedoch Schrägrisse auf, was etwa für

$$
\tau=\tau_{1}
$$




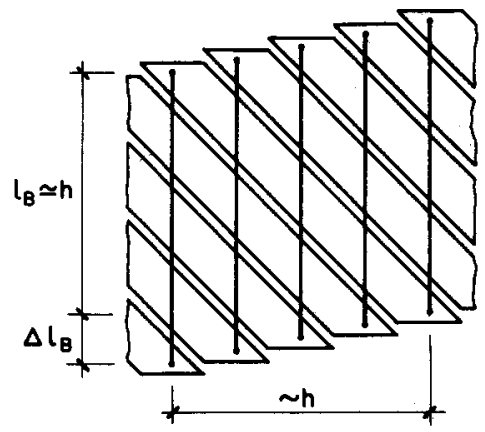

Bild 42

Modell zur Herleitung der Schubsteifigkeit

( $\tau$ nach ( 121 ), $\tau_{1}$ nach 3.11 respektive [9] ) der Fall ist, so können die Schubverformungen wesentlich zu einer Umlagerung der inneren Kräfte beitragen und müssen somit bei der Berechnung der Matrix-Koeffizienten berücksichtigt werden.

In Bild 42 ist ein mit $45^{\circ}$-Rissen kontinuierlich durchsetzter Balkenteil dargestellt. Dieser werde ausschliesslich durch eine Querkraft beansprucht. Sind als Schubarmierung nur Bügel vorhanden, so lässt sich năherungsweise die Schubsteifigkeit aus deren Verformungen herleiten. Der Schiebungswinkel ergibt sich wie folgt:

$$
\gamma=\frac{\Delta 1_{B}}{{ }_{B}} \cong \frac{\Delta I_{B}}{h}
$$

Setzen wir voraus, dass die Bügel nur elastische Verformungen erleiden, so gilt:

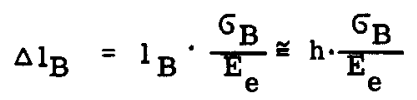


Die mittlere Bügelspannung $G_{B}$ wird

$$
\sigma_{B} \cong \frac{B_{45}}{F_{B}} \cdot \frac{t}{h},
$$

wenn $B_{45}$ die im $45^{\circ}$-Riss übertragene Querkraft bedeutet. Sollte $B_{45}$ entsprechend dem Modell Bild 42 gleich der Querkraft gesetzt werden, so zeigen anderseits die Untersuchungen [9], dass sowohl im Biegeschub- wie auch im Diagonalschubbereich Randzonen des Querschnitts einen Teil der Querkraft übertragen. Es kann wie im Abschnitt 3.22 gesetzt werden:

$$
B_{45}=Q-Q_{C},
$$

mit $Q=\tau b_{0} h$ nach ( 121$)$ und $Q_{C}=\tau_{1} b_{0} h$ nach ( 142$)$. Damit wird der Schiebungswinkel:

$$
\gamma=\frac{Q-Q_{C}}{F_{B} \cdot E^{\cdot\left(\frac{h}{t}\right)}}
$$

Andererseits gilt nach der tiblichen Schreibweise:

$$
\gamma=\frac{\mathrm{Q}}{\mathrm{GF}}
$$

Aus ( 200 ) und ( 201 ) wird die Schubsteifigkeit:

$$
G^{\prime}=\frac{F_{B} \cdot E_{e} \cdot\left(\frac{h}{t}\right)}{\lambda},
$$

mit

$$
\lambda=1-\tau_{1} / T
$$


Damit ist es gelungen, die Schubsteifigkeit in analoger Weise wie die Biegesteifigkeit nach ( 194 ) darzustellen. Der Keoffizient $\lambda$ unter dem Bruchstrich berücksichtigt - analog $\mathrm{zu} \kappa$ - wiederum die Abhängigkeit der Steifigkeit von der Höhe der Beanspruchung. In Bild 43 ist $\lambda$ dargestellt. Da nach [9] stets $\tau=5 \tau_{1}$ ist und andererseits für $\tau=\tau_{1}$ keine Schrägrissse zu erwarten sind, gilt für $\lambda$ :

$$
0,8 \geq \lambda \geq 0
$$

Dem entspricht für die Steifigkeit der Variationsbereich

$$
\frac{F_{B} \cdot E_{e} \cdot\left(\frac{h}{t}\right)}{0,8} \leq G F^{\prime} \leq \infty .
$$

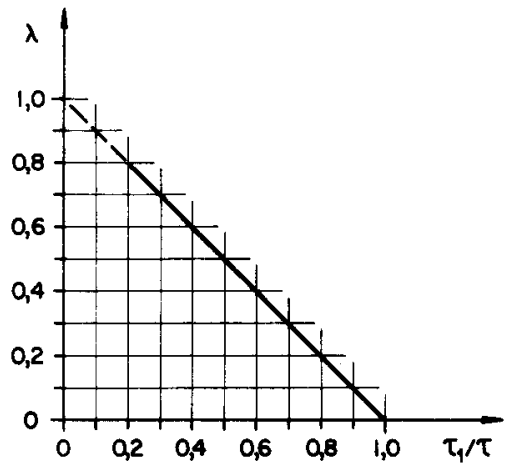

Bild 43

Koeffizient zur Bestimmung der Schubsteifigkeit im Bereich elastischer Verformungen der Bügelarmierung 
Grundsătzlich trăgt ausser der Verlăngerung der Bügel auch die Zusammendrückung der Betondruckstreben zu den Schubverformungen bei. Der Beitrag des Betons übersteigt jedoch selten $20 \%$ von demjenigen der Bügelarmierung. Zudem wird mit ( 199 ) respektive ( 144 ) die effektive Bügelbeanspruchung meist etwas überschätzt. Diese beiden Einflüsse kompensieren sich im allgemeinen weitgehend.

Wie für die Biegesteifigkeit ist auch für die Bestimmung der Schubsteifigkeit die Beanspruchung des betrachteten Zustandes massgebend. Die Schwierigkeit, dass diese Beanspruchung sich erst als Resultat der vorzunehmenden Berechnung ergibt, stellt sich oft auch hier ein. Hie und da kann deshalb nur ein iteratives Vorgehen ein befriedigendes Ergebnis liefern. Bei jeder Voraussage der Schubverformungen beziehungsweise der Schubsteifigkeit von Stahlbetonbalken kann es sich jedoch meist nur um eine Abschätzung der Grössenordnung handeln, da das Schubverhalten bekanntlich sehr komplex und rechnerisch sehr schwierig zu erfassen ist. Eine allzu differenzierte Berechnung würde deshalb in vielen Fällen nur zu einer Scheingenauigkeit führen.

\subsection{Ausdehnung der plastischen Gelenke}

Hier soll noch kurz eine weitere, bisher nicht berücksichtigte Besonderheit besprochen werden.

Die einfache Plastizitätstheorie setzt voraus, dass sich die plastischen Verformungen eines Gelenkes auf einen einzigen Querschnitt konzentrieren, das plastische Gelenk somit nur eine verschwindend kleine Ausdehnung hat. Zwischen den Gelenken sollen sich die Stäbe rein elastisch verformen. Bei Stahlbetonbalken erstrecken sich jedoch die plastischen Gelenie oft über einen Bereich, dessen Länge bis zu $2 \mathrm{~h}$ oder noch mehr betragen kann. In den vorangehend definierten kritischen Gelenkwinkeln $\Theta_{\mathbf{k r}}$ sind neben den plastischen auch die elastischen Verformungen dieses Bereiches enthalten. Ausserdem werden - genau wie in der einfachen Plastizitätstheorie - bei der Berechnung der erforderlichen Gelenkwinkel $\theta_{\mathrm{er}}$, respektive der Matrixkoeffizienten $\alpha_{i k}$, die elastischen Verformungen über die gesamten Stäbe, inklusive allfällige Gelenkbereiche, berücksichtigt. Die Voraussetzungen für den Vergleich von $\Theta_{\mathrm{kr}}$ und $\Theta_{\mathrm{er}}$ beziehungsweise deren Berechnung 
stimmen somit nicht voll mit den Gegebenheiten überein. Dem kann näherungsweise abgeholfen werden, indem der elastische Anteil von $\theta_{\mathbf{k r}}$, nämlich $\theta_{\mathrm{el}}$, wie folgt abgeschätzt wird:

$$
\theta_{\mathrm{el}} \cong \frac{\varepsilon_{f} \cdot 1 \mathrm{p}}{h-z}
$$

Darin bedeutet $l_{p}$ die Länge des Bereiches, über welchen sich das plastische Gelenk erstreckt, das heisst plastische Stahldehnungen auftreten.

Die Bedingung ( 1 ) kann damit wie folgt angeschrieben werden:

$$
\Theta_{\mathrm{kr}}-\Theta_{\mathrm{el}} \geq \Theta_{\mathrm{er}}
$$

Die Reduktion von $\theta_{\mathrm{kr}}$ durch $\theta_{\text {el }}$ ist allerdings meist nicht wesentlich. Auch ist der Abzug $\Theta_{\mathrm{el}}$ angesichts der in vielen Fällen schlechten Genauigkeit, welcher eine Voraussage von $\Theta_{\mathrm{kr}}$ unterliegt, oft problematisch und kann daher meist vernachlässigt werden. 


\section{ZUSAMMENFASSUNG}

\subsection{Einleitung}

Die plastizitätstheoretische Berechnung statisch unbestimmter Tragwerke berïcksichtigt die Tatsache, dass bis zum Bruch bedeutende Umlagerungen der inneren Schnittkräfte eintreten können. Solche Umlagerungen setzen das Entstehen plastifizierter Bereiche, sogenannter plastischer Gelenke, voraus. Eine vollständige Schnittkraftumlagerung, respektive das Erreichen der Traglast gemäss der einfachen Plastizitätstheorie, kann nur erfolgen, sofern in den plastischen Gelenken eine genügende Verformbarkeit vorhanden ist. Dies ist bei Stahlbetontragwerken nicht immer der Fall. Gelegentlich tritt ein vorzeitiger Bruch auf, da der Baustoff Stahlbeton ziemlich spröde ist.

Der Zweck der vorliegenden Arbeit ist, bei statisch unbestimmten Stahlbetonbalken die Verformungen in plastischen Gelenken zu untersuchen. In jedem plastischen Gelenk können die folgenden zwei Gelenkwinkel definiert werden:

$\begin{array}{ll}\theta_{\mathbf{k r}}: & \begin{array}{l}\text { Kritischer Winkel, das heisst der Gelenkwinkel beim Eintreten } \\ \text { eines kritischen Zustandes (Versagen) im Gelenk; }\end{array} \\ \theta_{\text {er }}: & \begin{array}{l}\text { Erforderlicher Winkel, das heisst der für eine vollstăndige Um- } \\ \text { lagerung der inneren Kräfte erforderliche Gelenkwinkel. }\end{array}\end{array}$

Der kritische Gelenkwinkel charakterisiert somit das Verformungsvermögen eines plastischen Gelenkes. Dieses Verformungsvermögen wird auch Deformationsoder Rotationsfăhigkeit plastischer Gelenke genannt. $\Theta_{k r}$ hängt nur von den lokaIen Gegebenheiten im Bereiche des Gelenkes ab. Der für eine vollständige Umlagerung der Schnittkrăfte beziehungsweise zum Erreichen der Traglast erforderliche Gelenkwinkel hingegen wird von den Verformungen und damit von den Gegebenheiten des ganzen Tragwerkes beeinflusst. Soll eine vollständige Schnittkraftumlagerung eintreten können, so muss in jedem plastischen Gelenk die folgende Bedingung erfiillt sein: 


$$
\begin{gathered}
-161- \\
\theta_{\mathrm{kr}} \geq \theta_{\mathrm{er}}
\end{gathered}
$$

Um die komplizierten Verhältnisse in plastischen Stahlbeton-Gelenken $\mathrm{zu}$ erfassen, werden die neuartigen Modelle "Biegeriss-Gelenk" und "Schubriss-Gelenk" entwickelt. Dabei wird auf verschiedene, in der Stahlbetontheorie sonst übliche Voraussetzungen und Begriffe verzichtet (Ebenbleiben der Querschnitte, Krümmung, elastisches Materialverhalten). Denn beim Stahlbeton handelt es sich infolge der Rissebildung um einen Baustoff mit ausgeprägt diskontinuierlichem Formänderungsverhalten. Darauf haben entsprechende Modelle unbedingt Rücksicht zu nehmen.

\subsection{Das Biegeriss-Gelenk}

Typische Biegeriss-Gelenke sind in den Arbeiten [6] und [7] abgebildet. Da die Schubbeanspruchung verhältnismässig gering ist, sind nur Biegerisse vorhanden. Das Modell des Biegeriss-Gelenkes (Bild 1) setzt sich aus mehreren "Biegeriss-Elementen" (Bild 4) zusammen. Der Gelenkwinkel ergibt sich als Summe der einzelnen "Elementwinkel", für die mit guter Näherung die "Risswinkel" gesetzt werden dürfen.

Für die Verformungen in der Zugzone sind die folgenden Grössen wesentlich:

- Verbundspannung $\tau_{\mathbf{v}}$ zwischen Zugarmierung und Beton,

- Relativverschiebung $\mathbf{v}$ zwischen Zugarmierung und Beton,

- Spannung $\sigma_{e}$ in der Zugarmierung,

- Dehnung $\varepsilon_{e}$ in der Zugarmierung.

Erstes Ziel der Berechnung ist der Verlauf dieser Grössen längs der Zugarmierung, das heisst die Funktionen $\tau_{v}(x), v(x), \sigma_{e}(x), \varepsilon_{e}(x)$, vergleiche Bild 4. Hiefür bilden die experimentell $\mathrm{zu}$ ermittelnden

- Verbundgesetz $\tau_{v}(v)$ und

- Spannungs-Dehnungs-Diagramm $\varepsilon_{e}\left(\sigma_{e}\right)$ der Zugarmierung 
die Grundlagen (Bilder 6 und 7). Wegen der allgemeinen Form dieser beiden Funktionen muss die Integration der entstehenden Differentialausdrücke über jeden Stababschnitt $\Delta \mathrm{x}$ als Iteration mittels Differenzenbeziehungen durchgeführt werden. Zu einer Vereinfachung der Berechnung können jedoch graphische Tafeln aufgestellt werden. Dies wurde hier für Torstahl mit $\tau_{v}(v)=$ konst. durchgeführt (siehe Tafel 1). Solche Darstellungen enthalten sämtliche Grössen, welche die Beanspruchungen und Verformungen in der Zugzone eines Biegeriss-Elementes charakterisieren. Es kann als Funktion der übrigen Grössen auch der wichtige

$$
\text { Verbundkoeffizient }=\kappa=\frac{\text { mittlere Stahldehnung }}{\text { maximale Stahldehmung }}
$$

aufgenommen werden. Es wird von der Güte des Verbundes, der Form des Spannungs-Dehnungs-Diagrammes, der maximalen Stahldehnung im Riss, dem Stabdurchmesser und anderem mehr beeinflusst. Für handelsübliche hochwertige Armierungsstähle kann der Verbundkoeffizient unter Umständen bis etwa zum Wert 0,10 absinken, für glatten Rundstahl hingegen fast 1,0 betragen.

Auch die Verformungen in der Druckzone eines Biegeriss-Elementes sind ungleichmässig verteilt (Bild 11). Die Variation der Randstauchung ist hauptsächlich abhäng ig vom Verhältnis, gebildet durch den Abstand $z$ des Rissendes vom Druckrand und dem Rissabstand s. Sie kann mit einem entsprechenden Ansatz näherungsweise erfasst werden.

Die Rissweite ergibt sich als Summe der Verschiebungen $\mathrm{v}$ an den beiden Rissufern. Die "kritische Rissweite" in einem Biegeriss-Eelement ist dann vorhanden, wenn entweder in der Zugzone oder in der Druckzone ein kritischer Zustand erreicht worden ist. Für das Versagen der Zugarmierung wird der Beginn der Einschnürung als massgebend betrachtet. Dann entspricht die Dehnung im Riss mit guter Näherung der Gleichmassdehnung. Für das Versagen des Betons ist die maximale Randstauchung uiber dem Riss wesentlich. Der Einfluss einer umschnürenden Wirkung von Bügeln kann in der Rechnung berücksichtigt werden (Bilder 15 und 16).

Der kritische Winkel in einem Biegeriss-Gelenk setzt sich aus dem Risswinkel 
im kritischen Riss und allfälligen weiteren Risswinkeln in angrenzenden Biegeriss-Elementen zusammen, in denen kein Versagen eintritt. Entsprechend sind die einzelnen Rissweiten zu berechnen, das heisst ausgehend vom kritischen Riss und fortschreitend $\mathrm{zu}$ den übrigen Rissen, unter Benützung der jeweiligen Randbedingungen.

Eine Untersuchung über den Einfluss wichtiger Parameter zeigt, dass der kritische Winkel in einem Biegeriss-Gelenk infolge Versagen des Betons insbesondere abnimmt mit

- grösserer Querkraft und

- grösserem Rissabstand.

Der kritische Gelenkwinkel infolge Versagen der Zugarmierung hingegen wird kleiner mit

- grösserer Querkraft,

- grösserem Rissabstand,

- kleinerer Gleichmassdehnung,

- schlechteren Verfestigungseigenschaften,

- besseren Verbundeigenschaften,

- kleineren Stabdurchmessern.

Soll zum Beispiel die Gefahr für ein Versagen des Stahles vor demjenigen des Betons beurteilt werden, so muss der Einfluss all dieser Parameter beriicksichtigt werden. Falls verschiedene Stahlsorten bezüglich dieser Gefahr miteinander verglichen werden müssen, so sind nebst den Dehnungseigenschaften auch die Verfestigungs - und Verbundeigenschaften in Betracht zu ziehen.

Im Hinblick auf den Gebrauchszustand (Rissebildung) werden normalerweise gute Verbundeigenschaften und kleine Stabdurchmesser gefordert. Diese wirken sich jedoch - wie die obige Aufstellung zeigt - auf die Verformbarkeit ungünstig aus.

Die für das Biegeriss-Gelenk entwickelten Verfahren können anhand von Messresultaten aus den Versuchen [7] überprüft werden. Es zeigt sich, dass die aus der theoretischen Berechnung resultierenden Werte für die kritischen Gelenkwinkel einen sicheren untern Grenzwert darstellen. 
Schliesslich interessiert noch ein Vergleich zwischen den Ergebnissen aus der hier entwickelten Betrachtungsweise und den Verfahren, welche aufgrund der Hypothese vom Ebenbleiben der Querschnitte resultieren. Solange die Zugarmierung keine plastischen Deformationen erleidet, ergeben sich nur verhältnismässig geringe Fehler gegenüber dem tatsächlichen Verhalten. Sobald jedoch die Zugarmierung plastische Deformationen aufweist, kann die Hypothese vom Ebenbleiben der Querschnitte zu schwerwiegenden Fehlschlüssen führen.

Eine allgemeine Beurteilung der Deformationsfähigkeit des Biegeriss-Gelenkes ergibt, dass diese in hohem Masse von verschiedenen Parametern abhängt. Sie ist oft recht gross, bisweilen aber auch ungenügend klein. Besonders ungünstig wirkt sich eine verhältnismässig grosse Schubbeanspruchung aus, weil sich dann die plastischen Deformationen auf einen kleinen Bereich, bei grösserem. Rissabstand eventuell sogar auf den Bereich eines einzigen Biegerisses konzentrieren können. In solchen Fällen ist es nötig, sich über die Grösse des kritischen Gelenkwinkels Rechenschaft zu geben. Ist die Schubbeanspruchung jedoch gering, beziehungsweise stellt man zu Beginn der Untersuchung fest, dass in mehreren Rissen (mindestens drei) plastische Deformationen auftreten, so erübrigt sich meistens eine genauere Berechnung des kritischen Gelenkwinkels.

\section{3 Das Schubriss-Gelenk}

Typische Schubriss-Gelenke sind in den Arbeiten [6], [7] und [8] abgebildet. Sie treten vor allem dort auf, wo konzentrierte Einzelkräfte senkrecht zur Stabachse wirken, also insbesondere uiber $\mathrm{Zwischenstützen} \mathrm{von} \mathrm{Durchlauf-}$ trägern.

In Schubriss-Gelenken ist ausser dem Biegemoment noch eine erhebliche Schubbeanspruchung vorhanden, sodass sogenannte Biegeschubrisse entstehen. Diese verlaufen schräg auf die Stelle der Krafteinleitung hin. Im Modell des SchubrissGelenkes (Bild 31) ergibt sich der Gelenkwinkel - genau wie im Biegeriss-Gelenk - als Summe der einzelnen Risswinkel. 
Gewisse Schwierigkeiten bereitet die Bestimmung der innern Kräfte in den verschiedenen Elementen des Gelenkes (Bilder 32 und 33). Die Bügelarmierung und die Biegedruckzone übertragen gemeinsam die Querkraft. Deren Aufteilung beeinflusst die Kraft in der Zugarmierung. Zur Lösung der Probleme können jedoch Erkenntnisse aus den Versuchen [7] und [8], sowie aus der Arbeit

[9] herangezogen werden.

Die Verformungen der Zugzone zwischen zwei Biegeschubrissen sind ähnlich denjenigen in der Zugzone zwischen zwei Biegerissen (Bilder 34 und 17). Der Gelenkwinkel muss ebenfalls ausgehend vom breitesten Riss bei der Krafteinleitung und fortschreitend zu den übrigen Rissen - unter Benützung der jeweiligen Randbedingungen - ermittelt werden.

Im Vergleich zum Biegeriss-Gelenk ist im Schubriss-Gelenk eine grössere Anzahl von Versagensmöglichkeiten in Betracht zu ziehen. Solche sind:

- Versagen der Zugarmierung,

- Versagen des Betons (im Steg und am Druckrand),

- Versagen der Bügelarmierung,

- Versagen des Verbundes der Zugarmierung.

Die Berechnung der entsprechenden kritischen Gelenkwinkel stellt teilweise fast unlösbare Probleme. Bei einem Versagen der Zugarmierung, das nur selten vorkommen dürfte, können im wesentlichen die für das Biegeriss-Gelenk erarbeiteten Grundlagen übernommen werden. Schwiereiger hingegen ist die rechnerische Erfassung des Versagens des Betons. Während man aufgrund der üblichen Stahlbetonbiegetheorie geneigt ist, dem Versagen am Druckrand eine grosse Bedeutung zuzumessen, zeigen Versuche, dass dieses in einem Schubriss-Gelenk meist nicht wesentlich ist. Denn hohe Betonstauchungen treten nur in einem recht kleinen Bereich auf (Bild 36). Die entsprechenden Zerstörungen der Randfaser führen daher kaum zu einem Versagen im Gelenk. Bedeutsamer ist demgegenüber die Gefahr für ein Versagen des Stegbetons an der Wurzel der schiefen Druckstreben. Die Hauptbeanspruchung besteht aus schiefen Druckkräften. Die entsprechenden Betonspannungen können ein Mehrfaches derjenigen Werte erreichen, die im sogenannten "Diagonalschubbereich" (vergleiche [9]), wo die Schubrisse 
parallel verlaufen, etwa auftreten. Sie hägen fast ausschliesslich von der Grösse der Schubbeanspruchung ab. Für die Bestimmung des kritischen Gelenkwinkels sollte deshalb die Funktion $\Theta(Q)$, das heisst das Anwachsen des Gelenkwinkels mit zunehmender Querkraft (Bild 35), bekannt sein. Da die Festsetzung einer kritischen Grösse der schiefen Druckspannungen schwierig und auch die Ermittlung der Funktion $\Theta(Q)$ ausserordentlich aufwendig sind, ist eine $\mathrm{Be}-$ rechnung oder auch nur eine Abschätzung des kritischen Gelenkwinkels infolge Versagen des Stegbetons kaum möglich. Vielmehr muss ein solcher Bruch durch eine Begrenzung der Schubbeanspruchung vermieden werden.

Aehnliche Schwierigkeiten ergeben sich im Falle des Versagens der Bügelarmierung, das heisst bei Ueberschreiten der Streckgrenze in derselben. Um ein solches Versagen zu verhindern, muss der Bemessung der Bügelarmierung im Bereiche eines Schubriss-Gelenkes eine genügend grosse Querkraft zugrundegelegt werden.

Schliesslich kann auch ein ungenügender Verbund der Zugarmierung Ursache des Versagens in einem Schubriss-Gelenk sein. Die entsprechende Gefahr lässt sich durch ein einfaches Kriterium abschätzen. Sie ist bei den heutigen hochwertigen Armierungsstăhlen auch bei hohen Schubbeanspruchungen jedoch gering.

Es zeigt sich somit, dass eine Berechnung des kritischen Gelenkwinkels in Schubriss-Gelenken meist ausserordentlich aufwendig und zudem unsicher ist. Die Untersuchung weiterer Aspekte des Problems ergibt jedoch, dass sich eine solche Berechnung in vielen Fällen eribrigt. Betrachtet man nämlich den Verlauf der Kraft in der Zugarmierung, so zelgt sich, dass diese nur wenig variiert (Bild 37). Die Zugkraft in den Rissen verändert sich nicht etwa affin zur Momentenfläche - wie dies aufgrund der Hypothese vom Ebenbleiben der Querschnitte oft angenommen wird - sondern bedeutend weniger. Aehnlich verhalten sich die entsprechenden Dehnungen (Bild 38). Bei üblichen Verhältnissen entstehen somit in der Zugarmierung über einem grösseren Bereich plastische Deformationen, das heisst solche treten in mehreren Rissen auf. Dies bedeutet, dass der kritische Gelenkwinkel verhältnismässig gross ist. Er genügt in den meisten Fällen für eine Umlagerung der innern Kräfte. 
Betrachtet man die generelle Abhängigkeit des kritischen Gelenkwinkels in Biegeriss - und Schubriss-Gelenken von der Schubbeanspruchung (Bild 39), so stellt man fest, dass der ungünstigste Fall das schubbeanspruchte Biegeriss-Gelenk darstellt. Bei diesem konzentrieren sich die plastischen Deformationen auf einen relativ kleinen Bereich, eventuell auf nur einen einzigen Riss. Ist die Schubbeanspruchung jedoch derart, dass Biegeschubrisse entstehen, so erstrecken sich die plastischen Deformationen über einen bedeutend grösseren Bereich. Trotz stärkerer Verănderung des äusseren Momentes entstehen in mehr Rissen plastische Deformationen, wodurch der Gelenkwinkel bis zum Eintreten der verschiedenen Versagensfälle ganz beträchtlich anwachsen kann.

\subsection{Erforderliche Gelenkwinkel}

Bei der Berechnung derjenigen Gelenkwinkel, welche für eine vollständige Momentenumlagerung, das heisst für das Erreichen der Traglast, erforderlich sind, kann von den Begriffen und Voraussetzungen der einfachen Plastizitätstheorie ausgegangen werden. Wo es nötig und zweckmässig ist, müssen Modifikationen und Erweiterungen vorgenommen werden.

Die Zusammenhänge werden am Beispiel eines über 6 Felder durchlaufenden Trägers entwickelt, bei welchem in einem Innenfeld ein lokaler Mechanismus auftritt (Bild 40). Betrachtet man den Zustand unmittelbar beim Erreichen der Traglast, dann sind in den plastischen Gelenken gerade die gesuchten erforderlichen Gelenkwinkel vorhanden. Um diese berechnen zu können, müssen im Falle eines lokalen Mechanismus zu den plastischen Gelenken hinzu noch soviele weitere Gelenke eingeführt werden, dass ein statisch bestimmtes Grundsystem entsteht. Aus der entsprechenden Matrix können die erforderlichen Gelenkwinkel durch teilweise Inversion ermittelt werden. Gleichzeitig ergeben sich die Momente in den übrigen Gelenken.

Auf analoge Art lassen sich auch irgendwelche Zustände vor dem Erreichen der Traglast erfassen. Allgemein entsprechen sämtlichen Tragsystemen, welche bei steigender Lastintensität über die Fliesslast bis zur Traglast entstehen, ganz bestimmte Matrizen. Diese ergeben sich aus der Matrix des statisch bestimmten 
Grundsystems durch das Verfahren der Austauschschritte - bei entsprechender Wahl der Pivotelemente - auf einfache Weise.

Während das entwickelte Verfahren für beliebige Stabtragwerke unter den Voraussetzungen der einfachen Plastizitätstheorie gültig ist, gilt es bel Stahlbetonbalken zu beachten, dass die Momente in den plastischen Gelenken auch nach Ueberschreiten der Streckgrenze noch zunehmen können. Dies zwingt häufig zu einem iterativen Vorgehen bei der Berechnung der erforderlichen Gelenkwinkel. Ein anderes schwieriges Problem stellt die Bestimmung der Steifigkeiten dar, welche die Grösse der Matrixkoeffizienten massgeblich beeinflussen. Mit Hilfe des Verbundkoeffizienten $\kappa$ ist es möglich, die Biegesteifigkeit verhältnismässig zutreffend zu berechnen. Mehr Schwierigkeiten bereitet die Ermittlung der Schubsteifigkeit. Diese kann aus der Bügelbeanspruchung hergeleitet werden, doch handelt es sich meist nur um eine grobe Abschätzung der Grössenordnung. 


\section{SCHLUSSBEMERKUNG}

Wie die vorangehenden Untersuchungen zeigen, sind die Probleme, welche sich bei der plastizitätstheoretischen Berechnung von statisch unbestimmten Stahlbetonbalken ergeben, so geartet, dass vorläufig keine einfachen Regeln für die Praxis aufgestellt werden können. Die Deformationen plastifizierter Bereiche werden von einer Vielzahl von Parametern beeinflusst, sodass eine differenziertere Betrachtung der jeweiligen Gegebenheiten meist unumgänglich ist. Mit der Wirklichkeit gut übereinstimmende Rechenergebnisse können aber auch aufgrund der hier entwickelten Modelle und Verfahren nicht immer erwartet werden. Hier ging es vor allem um die vertiefte Einsichtnahme in komplizierte $\mathrm{Zu}$ sammenhänge. Das Hauptziel war - im Sinne einer Grundlagenforschung - zu erfassen und darzustellen, was in plastifizierten Bereichen von statisch unbestimmten Stahlbetonbalken bei einer Steigerung der Lastintensität bis zum Bruch vor sich geht. Infolge der naturgemässen Schwierigkeiten sind die Ergebnisse der Bemühungen teilweise nur qualitativer Natur. Wie sich zeigte, führt jedoch oft bereits die qualitative Erfassung der Verhältnisse, oder dann - wenn nötig eine zusätzliche grobe Abschätzung zu einer klärung der Probleme. Aus einer richtigen qualitativen Analyse resultieren auch Anregungen zu konstruktiven Massnahmen, die oft mit kleinem Aufwand erhebliche Vorteile bringen können. Zudem ist festzuhalten, dass Stahlbetontragwerke ohnehin meist eine erhebliche Verformbarkeit aufweisen. Eine weitere, intensive Forschung hat abzuklären, wann und inwieweit diese Verformbarkeit bei der Bemessung berücksichtigt werden soll. 


\section{ANHANG}

Numerische Angaben und Berechnungen zum Abschnitt "2.52 Vergleiche mit Versuchsresultaten"

\section{a) Balken A1}

Aus den Versuchen werden die folgenden Grössen benützt:

$\sigma_{\mathrm{s}}=3610 \mathrm{~kg} / \mathrm{cm}^{2}, \quad \beta_{\mathrm{z}}=4400 \mathrm{~kg} / \mathrm{cm}^{2}, \quad E_{\mathrm{e}}=2,07 \cdot 10^{6} \mathrm{~kg} / \mathrm{cm}^{2}$,

$\beta_{\mathrm{w}}=438 \mathrm{~kg} / \mathrm{cm}^{2}, \quad \lambda_{\mathrm{Gl}}=5,6 \%, \quad \mathrm{~h}=35,1 \mathrm{~cm}, \quad \mathrm{~h}^{\prime}=2,6 \mathrm{~cm}$,

$\mathrm{d} \cong 1,2 \mathrm{~cm}, \quad F=1,155 \mathrm{~cm}^{2}, \quad t=17,5 \mathrm{~cm}, \quad c=14,8 \mathrm{~cm}, \quad s \cong 17,5 \mathrm{~cm}$, $Q=1,76 \mathrm{t}$ (Laststufe 14).

Die Kennwerte für die Zugarmierung wurden in [7] der Tabelle 12 entnommen (Probestab Nr. 3).

Rechnerischer Winkel im ersten (kritischen) Riss infolge Versagen des Stahls:

$$
\begin{aligned}
& \tau_{v}^{*} \cong 0,15 \beta_{w}=0,15 \cdot 438 \cong 66 \mathrm{~kg} / \mathrm{cm}^{2} \\
& \Delta \sigma_{e t o t}=\tau_{v}^{*} \cdot \frac{4}{d} \cdot \frac{\mathrm{g}}{2}=66 \cdot \frac{4}{1,2} \cdot \frac{17,5}{2}=1925 \mathrm{~kg} / \mathrm{cm}^{2} \\
& \sigma_{f}=\sigma_{\mathrm{s}}=3610 \mathrm{~kg} / \mathrm{cm}^{2} \\
& \varepsilon_{\mathrm{f}}=\frac{\sigma_{f}}{E_{e}}=\frac{3610}{2,07 \cdot 10^{6}}=0,001743 \\
& \Delta \sigma_{e t o t}=\frac{\Delta \sigma_{e t o t}}{\sigma_{f}}=\frac{1925}{3610}=0,533 \\
& \sigma_{e \max }=\beta_{z}=4400 \mathrm{~kg} / \mathrm{cm}^{2} \\
& \sigma_{e \max }=\frac{\sigma_{e \max }}{\sigma_{f}}=\frac{4400}{3610}=1,219
\end{aligned}
$$


Dem Wert $\bar{\sigma}_{e \max }=1,219$ entspricht im normierten Spannungs-DehnungsDiagramm Bild 10

$\bar{\varepsilon}_{\text {emax }} \cong 20,6$

Für $\bar{\varepsilon}_{\mathrm{e} \max } \cong \frac{\lambda_{G l}}{\varepsilon_{f}}=\frac{0,056}{0,001743}=32,1$ wird jedoch

$\bar{\sigma}_{\text {e } \max }=1,247$. Das Spannungs-Dehnungs-Diagramm des im Balken A1 verwendeten Stahles weist somit eine geringere Verfestigung, das heisst ein kleineres Verhältnis $\beta_{z} / \sigma_{s}$ auf als das normierte Diagramm Bild 10. Trotzdem kann Tafel 1 năherungsweise verwendet werden. Man entnimmt daraus folgende Werte für $\overline{\mathbf{r}}$ :

$\overline{\mathrm{r}}\left(\bar{\sigma}_{\mathrm{e} \max }=1,219, \Delta \bar{\sigma}_{\text {etot }}=0,533\right)=2,177$

$\overrightarrow{\mathbf{r}}\left(\bar{\sigma}_{e \max }=1,247, \Delta \bar{\sigma}_{e \text { tot }}=0,533\right)=2,851$

Damit kann $\overrightarrow{\mathbf{r}}$ des tatsächlichen Spannungs-Dehnungs-Diagrammes wie folgt geschätzt werden:

$\overline{\mathbf{r}} \cong 2,4$

Weiter ergibt sich:

$\begin{aligned} v_{\max }=\frac{w_{1 \mathrm{kre}}}{2}=\frac{\mathrm{d}}{4 \tau_{\mathrm{v}}^{*}} \cdot \varepsilon_{\mathrm{f}} \cdot \sigma_{\mathrm{f}} \cdot \overline{\mathbf{r}} & =\frac{1,2}{4 \cdot 66} \cdot 0,001743 \cdot 3610 \cdot 2,4 \\ & =0,0687 \mathrm{~cm}\end{aligned}$

$\mathrm{w}_{1 \mathrm{kre}}=0,1374 \mathrm{~cm}$

$\mathrm{z}=\mathrm{h}^{\prime}=2,6 \mathrm{~cm} ; \mathrm{h}-\mathrm{z}=35,1-2,6=32,5 \mathrm{~cm}$

$\omega_{1 \mathrm{kre}}=\frac{\mathrm{w}_{1 \mathrm{kre}}}{\mathrm{h}-\mathrm{z}}=\frac{0,1374}{32,5}=0,00423$ 
Zugehöriges $\kappa$ :

$$
\kappa=\frac{\bar{r}}{\bar{\varepsilon}_{\text {e max }} \cdot \Delta \bar{\sigma}_{\text {etot }}}=\frac{2,4}{32,1 \cdot 0,533}=0,14
$$

Rechnerischer Winkel im zweiten Riss bei Versagen des Stahles im ersten (kritischen) Riss:

$$
\begin{aligned}
& F_{e}=2 \cdot F=2 \cdot 1,155=2,31 \mathrm{~cm}^{2} \\
& y=h-\frac{z}{2}+\frac{\mu^{\prime}}{\mu} \cdot \frac{\sigma_{f}^{\prime}}{\sigma_{f}} \cdot\left(\frac{z}{2}-h^{\prime}\right)=35,1-\frac{2,6}{2}=33,8 \mathrm{~cm} \\
& \sigma_{e \max }=\beta_{z}-\frac{Q \cdot s}{F_{e} \cdot y}=4400-\frac{1760 \cdot 17,5}{2,31 \cdot 33,8}=4006 \mathrm{~kg} / \mathrm{cm}^{2} \\
& \bar{\sigma}_{e \max }=\frac{\sigma_{e \max }}{\sigma_{f}}=\frac{4006}{3610}=1,125 \\
& \Delta \bar{\sigma}_{e \text { tot }}=0,533 \text { (wie im ersten Riss) }
\end{aligned}
$$

Für $\bar{G}_{\text {emax }}=1,125$, das heisst für $\bar{\varepsilon}_{e \max }=8,60$ ist die Abweichung des tatsächlichen Spannungs-Dehnungs-Diagrammes vom normierten Diagramm Bild 10 nur gering. Aus Tafel 1 wird $\overline{\mathbf{r}}=0,95$. Der geschätzte Wert beträgt:

$$
\overline{\mathbf{r}} \cong 1,01
$$

Weiter wird:

$$
\begin{aligned}
& v_{\max }=\frac{w_{2 \mathrm{e}}}{2}=\frac{\mathrm{d}}{4 \tau_{\mathrm{v}}^{*}} \cdot \varepsilon_{\mathrm{f}} \cdot \sigma_{\mathrm{f}} \cdot \overline{\mathbf{r}}=\frac{1,2}{4 \cdot 66} \cdot 0,001743 \cdot 3610 \\
& \cdot 1,01=0,0289 \mathrm{~cm} \\
& w_{2 \mathrm{e}}=0,0578 \mathrm{~cm}
\end{aligned}
$$




$$
w_{2 \mathrm{e}}=\frac{\mathrm{w}_{2 \mathrm{e}}}{\mathrm{h}-\mathrm{z}}=\frac{0,0578}{32,5}=0,00178
$$

Zugehöriges $\kappa$ (mit geschätztem $\left.\bar{\varepsilon}_{\mathrm{emax}} \cong 10\right)$ :

$$
\kappa=\frac{\bar{r}}{\vec{\varepsilon}_{\text {e } \max }{ }^{-} \Delta \bar{\sigma}_{\text {etot }}}=\frac{1,01}{10 \cdot 0,533}=0,19
$$

Rechnerischer kritischer Winkel im Messabschnitt $19 / 20$ bei Versagen des Stahles:

$$
\omega_{19 / 20 \mathrm{kre}}=\omega_{1 \mathrm{kre}}+\omega_{2 \mathrm{e}}=0,00423+0,00178=0,00601
$$

Es muss noch kontrolliert werden, ob auch rechnerisch Versagen des Stahls massgebend ist:

Rechnerischer Winkel im ersten (kritischen) Riss infolge Versagen des Betons:

$$
\begin{aligned}
& \frac{z}{s}=\frac{2,6}{17,5}=0,1486 \\
& \mu_{q}=\frac{F_{q}}{t \cdot c}=\frac{0,51}{17,5 \cdot 14,8}=0,00197 \\
& A=\left[\frac{c-t / 2}{c}\right]^{2}=\left[\frac{14,8-17,5 / 2}{14,8}\right]^{2}=0,167 \\
& \varepsilon_{b r}=\varepsilon_{b r 0} \cdot\left[\frac{\beta_{w 0}}{\beta_{w}}\right]^{1 / 3}+1,4 \cdot A \cdot \mu_{q} \cdot\left[\frac{\beta_{w 0}}{\beta_{w}}\right]= \\
& =0,004\left[\frac{250}{438}\right]^{1 / 3}+1,4 \cdot 0,167 \cdot 0,00197 \cdot\left[\frac{250}{438}\right]=0,00358
\end{aligned}
$$




$$
\begin{array}{r}
\omega_{1 \mathrm{krb}}=\varepsilon_{b r} \cdot \frac{\mathrm{s}}{\mathrm{z}} \cdot\left[\frac{\mathrm{z}}{\mathrm{s}}\right]^{2 / 3}=0,00358 \cdot \frac{17,5}{2,6} \cdot 0,1486^{2 / 3}= \\
=0,00676
\end{array}
$$

Da $\omega_{1 \mathrm{kre}}<\omega_{1 \mathrm{krb}}$, ist Versagen des Stahles massgebend.

Zur Vollstăndigkeit wird jedoch noch angegeben, wie die Berechnung des Winkels im zweiten Riss vor sich gehen müsste, wenn im ersten Riss Versagen des Betons massgebend wăre:

$$
\begin{aligned}
& w_{1 \mathrm{krb}}=\omega_{1 \mathrm{krb}} \cdot(\mathrm{h}-\mathrm{z}) \\
& v_{\max }=\frac{w_{1 \mathrm{krb}}}{2} \\
& \bar{r}=v_{\max } \cdot \frac{4 \tau_{v^{*}}}{d \cdot \varepsilon_{\mathrm{f}} \cdot \sigma_{f}} \\
& \Delta \sigma_{\text {etot }}=\tau_{\mathrm{v}}^{*} \cdot \frac{4}{d} \cdot \frac{s}{2} \cdot \frac{1}{\sigma_{f}}
\end{aligned}
$$

Aus Tafel 1 folgt $\bar{\sigma}_{e \max }$ im kritischen Riss.

Daraus erhăl man mit $(30)$ und $(69)$ beziehungsweise $(71,72)$ den Wert für $\bar{\sigma}_{\text {e } \max }$ im zweiten Riss. Der entsprechende Winkel im zweiten Riss wird dann genau gleich berechnet, wie wenn im kritischen Riss Versagen des Stahles erfolgt wäre.

Zum Vergleich wird der Winkel 19/20 für Versagen der Zugarmierung noch nach dem verfeinerten Verfahren gemuss 2.41 b) berechnet. Der Einfluss der Querkraft auf den Verlauf der Stahldehnungen zwischen den Rissen wird hier also berilcksichtigt.

Die Differenz der Zugkräfte im ersten und zweiten Riss betrăgt: 
$\Delta \mathrm{Z}=\frac{\mathrm{Q} \cdot \mathrm{s}}{\mathrm{y}}=\frac{1760 \cdot 17,5}{33,8}=811 \mathrm{~kg}$

Die Stelle zwischen dem ersten und dem zweiten Riss, wo die Verbundspannungen und die Verschiebungen ihr Vorzeichen wechseln, ergibt sich mit

$q_{1,2}=\frac{\Delta z}{2 \tau_{v}{ }^{*} \cdot \pi \cdot \Sigma d}=\frac{911}{2 \cdot 66 \cdot \pi \cdot 2,4}=0,92 \mathrm{~cm}$

zu

$x_{\max 1,2}=\frac{s}{2}+q_{1,2}=\frac{17,5}{2}+0,92=9,67 \mathrm{~cm}$.

Weiter wird:

$$
\begin{aligned}
& \Delta \bar{G}_{\text {etot } 1,2}=\tau_{v} * \frac{4}{d} \cdot x_{\max 1,2}=66 \cdot \frac{4}{1,2} \cdot 9,67= \\
& 2128 \mathrm{~kg} / \mathrm{cm}^{2} \\
& \Delta \bar{G}_{\text {etot } 1,2}=\frac{\Delta \sigma_{\text {etot } 1,2}}{\sigma_{f}}=\frac{2128}{3610}=0,590
\end{aligned}
$$

Aus Tafel 1 ist ersichtlich, dass $\overline{\mathbf{r}}$ gegenüber der früheren Berechnung etwa um 0,04 grösser wird:

$\bar{r}_{1,2} \cong 2,40+0,04=2,44$

Damit erhălt man:

$$
\begin{array}{r}
v_{\max 1,2}=\frac{d}{4 \tau_{v}^{*}} \cdot \varepsilon_{f} \cdot \sigma_{f} \cdot \bar{r}_{1,2}=\frac{1,2}{4 \cdot 66} \cdot 0,001743 \cdot 3610 \cdot 2,44 \\
=0,0698
\end{array}
$$

Unter der Voraussetzung, dass $w_{1 \mathrm{kre}} \cong 2 \cdot v_{\max 1,2}$, wird: 


$$
\begin{aligned}
& w_{1 \mathrm{kre}}=0,1396 \mathrm{~cm} \\
& w_{1 \mathrm{kre}}=\frac{w_{1 \mathrm{kre}}}{\mathrm{h}-\mathrm{z}}=\frac{0,1396}{32,5}=0,00430
\end{aligned}
$$

Dieser Wert liegt 1,7\% über demjenigen, welcher vorher berechnet wurde.

Rechnerischer Winkel im zweiten Riss bei Versagen des Stahles im ersten (kritischen) Riss:

Vorerst ergibt sich die Verschiebung auf der einen Seite des Risses wie folgt:

$$
\begin{aligned}
& x_{\max 2,1}=\frac{s}{2}-q_{1,2}=\frac{17,5}{2}-0,92=7,83 \mathrm{~cm} \\
& \Delta G_{\text {etot } 2,1}=\tau_{v}^{*} \cdot \frac{4}{d} \cdot x_{\max 2,1}=66 \cdot \frac{4}{1,2} \cdot 7,83= \\
& \qquad \bar{\sigma}_{\text {etot } 2,1}=\frac{1722 \mathrm{~kg} / \mathrm{cm}^{2}}{(26 \mathrm{a})} \\
& \sigma_{\mathrm{f}}
\end{aligned}
$$

$\overline{\mathbf{r}}$ wird 0,023 kleiner als früher:

$$
\begin{aligned}
& \bar{r}_{2,1} \cong 1,01-0,023=0,987 \\
& v_{\max 2,1}=\frac{d}{4 \tau_{v}^{*}} \cdot \varepsilon_{f} \cdot \sigma_{f} \cdot \bar{r}_{2,1}=\frac{1,2}{4 \cdot 66} \cdot 0,001743 \cdot 3610 \cdot 0,987 \\
& =0,0282 \mathrm{~cm}(32)
\end{aligned}
$$

Die Verschiebung auf der andern Seite des zweiten Risses ergibt sich wie folgt:

$$
q_{2,3}=q_{1,2}=0,92 \mathrm{~cm}
$$


$x_{\max 2,3}=\frac{s}{2}+q_{2,3}=x_{\max 1,2}=9,67 \mathrm{~cm}$

$\Delta \bar{\sigma}_{\text {etot } 2,3}=\Delta \bar{G}_{\text {etot } 1,2}=0,590$

$\overline{\mathbf{r}}$ wird 0,020 grösser als früher:

$\overline{\mathrm{r}}_{2,3} \cong 1,01+0,02=1,03$

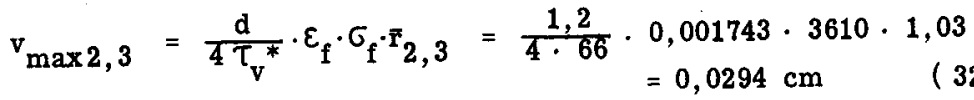

Im zweiten Riss beträgt somit die Rissweite beim Versagen des Stahles im ersten Riss:

$\mathrm{w}_{2 \mathrm{e}}=\mathrm{v}_{\max 2,1}+\mathrm{v}_{\max 2,3}=0,0282+0,0294=0,0576 \mathrm{~cm}$

Der entsprechende Winkel beträgt:

$\omega_{2 e}=\frac{w_{2 e}}{h-z}=\frac{0,0576}{32,5}=0,00177$

Man erkennt, dass die Verschiebungen beidseits des zweiten Risses wohl um geringe Beträge grösser beziehungsweise kleiner werden als nach der früheren Berechnung. Auf die Summe derselben, das heisst auf die Rissweite, und damit auf den Risswinkel, wirkt sich das variable Moment jedoch praktisch nicht aus.

Rechnerischer kritischer Winkel im Messabschnitt 19/20 bei Versagen des Stahles:

$$
\omega_{19 / 20 \mathrm{kre}}=\omega_{1 \mathrm{kre}}+\omega_{2 \mathrm{e}}=0,00430+0,00177=0,00607
$$

Dieser Wert liegt nur $1 \%$ über dem früher berechneten. Obwohl hier die Querkraft verhältnismässig gross war, entstanden somit durch die Berücksichtigung 
des variablen Momentes nur sehr geringe Abweichungen zum Ergebnis der vorherigen Berechnung nach $2.41 \mathrm{a})$. Diese kann als für praktische Zwecke genügend genau angesehen werden.

\section{b) Balken A2}

Aus [7] werden die folgenden Versuchsgrössen benützt, wobei die Kennwerte der Zugarmierung wiederum der dortigen Tabelle 12 (Probestab Nr. 4) entnommen sind:

$$
\begin{aligned}
& \sigma_{\mathrm{s}}=3850 \mathrm{~kg} / \mathrm{cm}^{2}, \quad \beta_{\mathrm{z}}=4560 \mathrm{~kg} / \mathrm{cm}^{2}, \quad \mathrm{E}_{\mathrm{e}}=2,06 \cdot 10^{6} \mathrm{~kg} / \mathrm{cm}^{2}, \\
& \beta_{\mathrm{w}}=438 \mathrm{~kg} / \mathrm{cm}^{2}, \quad \lambda_{\mathrm{Gl}}=6,6 \%, \quad \mathrm{~h}=35,1 \mathrm{~cm}, \quad \mathrm{~h}^{\prime}=2,6 \mathrm{~cm}, \\
& \mathrm{~d} \cong 1,2 \mathrm{~cm}, \quad \mathrm{~F}=1,150 \mathrm{~cm}^{2}, \quad \mathrm{t}=17,5 \mathrm{~cm}, \quad \mathrm{c}=14,8 \mathrm{~cm}, \\
& \mathrm{~s} \cong 17,5 \mathrm{~cm}, \mathrm{Q}=0,89 \mathrm{t} \text { (Laststufe } 16 \text { ). }
\end{aligned}
$$

Der rechnerische Winkel wird auf dieselbe Art und Weise ermittelt wie beim Balken A1.

\section{c) Balken A3}

Es können die gleichen Versuchsgrössen verwendet werden wie für die Berechnungen beim Balken A2, mit Ausnahme der Querkraft. Diese betrug hier bei Laststufe 27 nur 0,16 t.

Der rechnerische Winkel ergibt sich analog wie beim Balkon A1. 


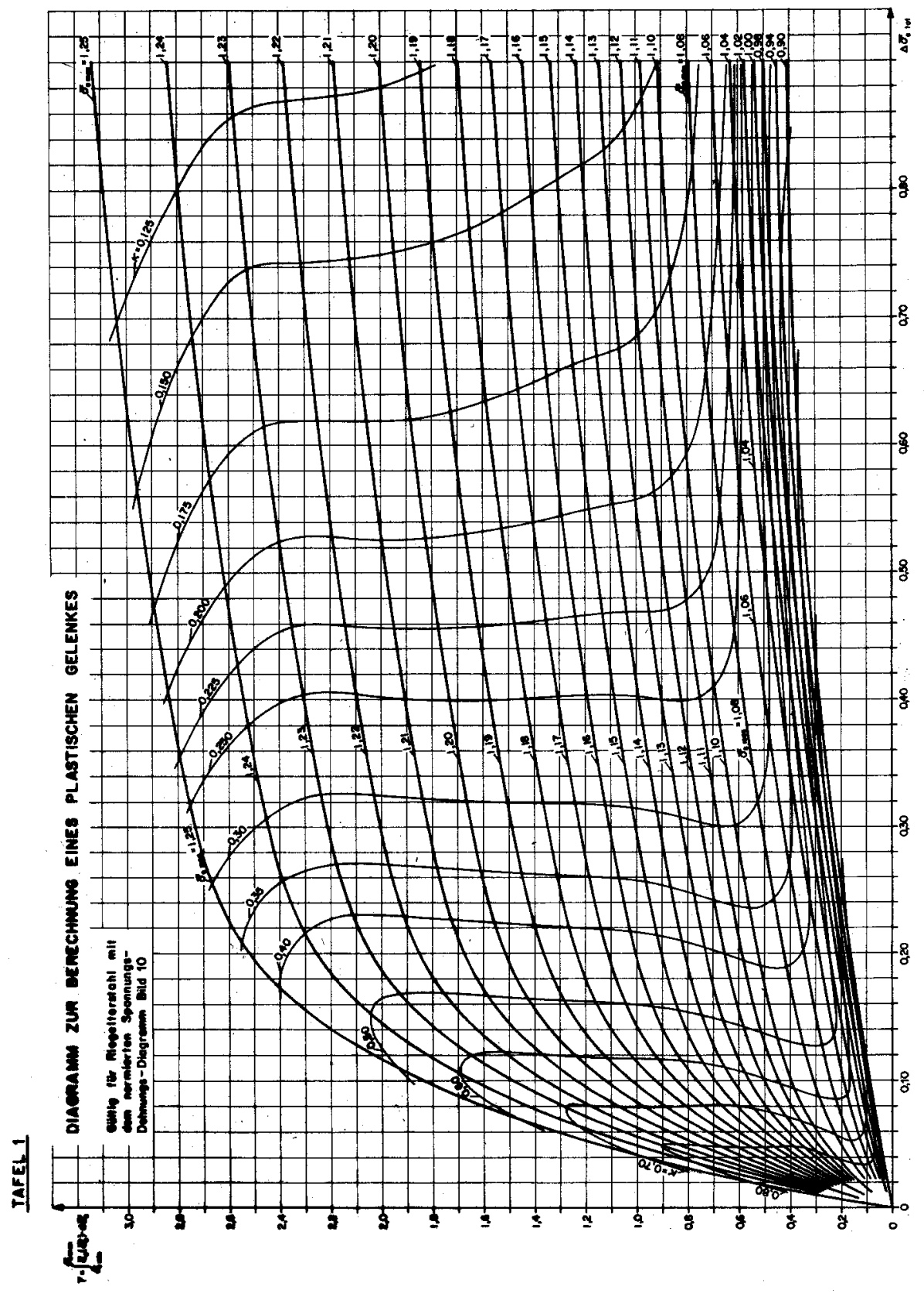




\section{BEZEICHNUNGEN}

Längen, Flächen und Querschnittswerte

a Hebelarm der Kraft $Q$ (Bild 32)

$a_{D} \quad$ Abstand der Druckfläche $F_{D}$ vom Druckrand

b Breite der Druckzone

$b_{0} \quad$ Stegbreite bei $T$ - und I-Querschnitten

$b_{a} \quad$ Breite, ubber welche eine Einzelkraft eingeleitet wird

$b_{D} \quad$ Breite der Druckfläche $F_{D}$

c Breite eines die Biegedruckzone umschnürenden Bügels (Bild 15)

d Durchmesser eines Armierungsstabes

e Hebelarm der Kraft B (Bild 32)

h Statische Nutzhöhe im Querschnitt

h' Abstand der Druckarmierung vom Druckrand

$\mathrm{h}_{\mathrm{F}} \quad$ Höhe eines Druckflansches

$l_{B} \quad$ Bügellänge

$l_{p} \quad$ Länge des Bereiches, über den sich ein plastisches Gelenk erstreckt

m Hebelarm der Kraft K (Bild 32)

q Abstand des Nullpunktes der Relativverschiebung zwischen Zugarmierung und Beton von der Mitte zwischen zwei Rissen

s Rissabstand

t Bügelabstand

$\mathrm{u} \quad$ Umfang eines Armierungsstabes: $u=\pi \cdot d$

$v \quad$ Relativverschiebung der Zugarmierung gegeniber dem Beton

$v_{\max }$ Maximale Relativverschiebung der Zugarmierung gegenüber dem Beton im Bereiche eines Risses

w Rissweite auf der Höhe der Zugarmierung

$w_{k r}$ Rissweite beim Versagen der Zugarmierung

$\mathrm{w}_{\mathrm{krb}} \quad$ Rissweite beim Versagen des Betons

w' $^{\prime} \quad$ Verkürzung der Betonfaser von der Länge $s$ am Druckrand im Bereiche eines Biegerisses 


\section{Lauf-Koordinate}

Abstand des Nullpunktes der Relativverschiebung zwischen Zugarmierung und Beton vom Riss

Hebelarm der innern Kräfte in Zug - und Druckzone

Distanz vom Ende eines Biegerisses bis zur Druckkante

Distanz vom Ende eines Biegeschubrisses bis zur Druckkante

Querschnittsfläche eines Stabes der Längsarmierung

F. Modifizierte Querschnittsfläche eines Balkens zur Berechnung der Schubverformungen

\section{Querschnittsfläche eines Stegbiigelelementes}

Querschnittsfläche einer schiefen Druckstrebe (Bereich zwischen zwei Biegeschubrissen)

Querschnittsfläche der Zugarmierung

Querschnittsfläche der Druckarmierung

Betonzugfläche

Querschnittsfläche eines die Biegedruckzone umschnürenden Bügelstabes

Widerstandsmoment des ungerissenen Querschnitts

Trägheitsmoment

id Ideelles Trägheitsmoment

Kräfte und Momente

B Resultierende der Bügelkräfte in einem Biegeschubriss neben dem Rand der Krafteinleitung (Bild 32)

$B_{45}$ Resultierende der Bügelkräfte in einem $45^{\circ}$-Biegeschubriss neben dem Rand der Krafteinleitung

C Parallel zur Balkenaxe wirkende Druckkraft in der Biegedruckzone (Bild 32)

D Druckkraft im Bereich zwischen zwei Biegeschubrissen

K Senkrecht zur Balkenaxe wirkende Querkraft in der Biegedruckzone (Bild 32) 


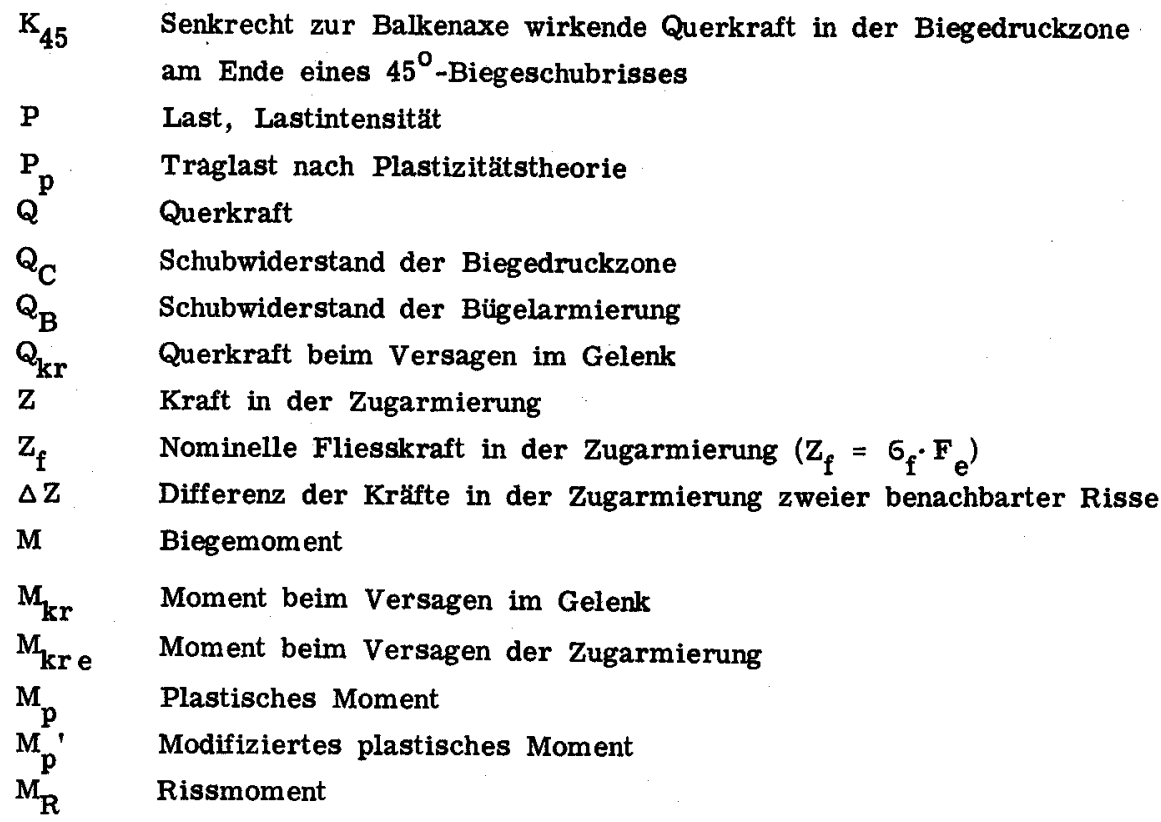

Festigkeitswerte und Spannungen

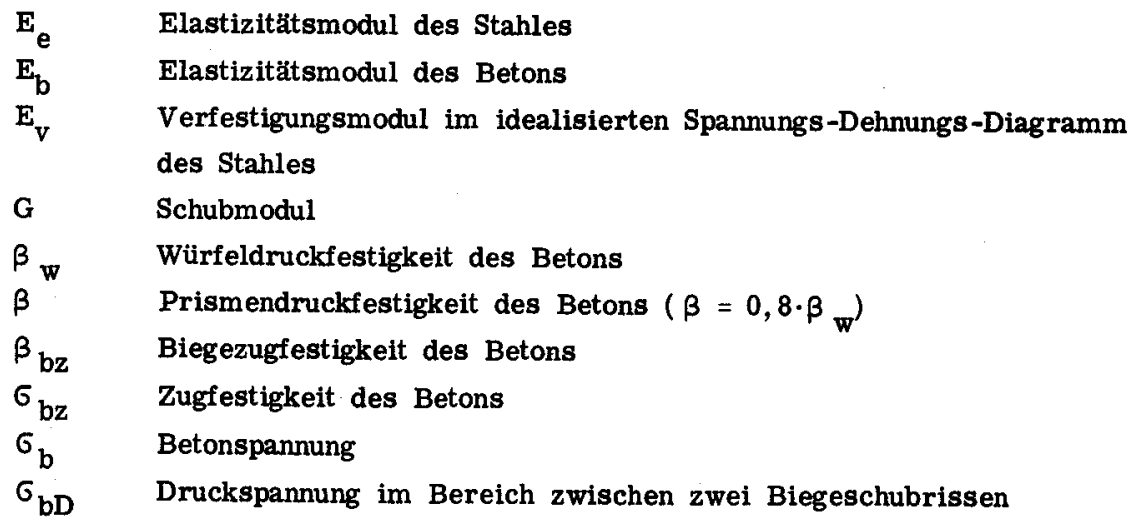


$\sigma_{\mathrm{s}}$

$\sigma_{\text {sB }}$

$\sigma_{\mathrm{f}}$

$\sigma_{\mathrm{f}}^{\prime}$

$\beta_{z}$

$\sigma_{\mathrm{e}}$

$\bar{\sigma}$

$\sigma_{\text {e } \max }$

$G$ emin

$\Delta \sigma_{\text {etot }}$

$\sigma_{\text {B }} \quad$ Spannung in der Bügelarmierung

$\tau \quad$ Nominelle Schubspannung: $\tau=Q / b_{0} h$

$\tau_{1} \quad$ Nominelle Schubspannung, bei deren Ueberschreiten Schrägrisse zu

erwarten sind, beziehungsweise ein Schubriss-Gelenk auftritt (3.11), sowie

$\tau_{1} \quad$ Nominelle Schubspannung zur Definition des Schubwiderstandes der Biegedruckzone (3.22)

$\tau_{\mathbf{v}} \quad$ Verbundspannung $z$ wischen Stahl und Beton

$\tau_{\mathrm{v}}^{*} \quad$ Konstant angenommene Verbundspannung

$\tau_{\text {vR }} \quad$ Mittlere Verbundspannung zur Berechnung des Rissabstandes

Matrix - Vektoren

$\begin{array}{ll}\bar{a} & \text { Vektor der abhängigen Variablen (Randspalte links) } \\ \overline{\mathrm{b}} & \text { Vektor der unabhängigen Variablen (Kopfzeile) } \\ \overline{\mathrm{a}}, & \text { Transponierter Vektor der abhängigen Variablen } \\ \overline{\mathrm{b}}^{\prime} & \text { Transponierter Vektor der unabhängigen Variablen }\end{array}$


Dehnungen, Krümmungen und Winkel

$\varepsilon_{\mathrm{e}} \quad$ Dehnung in der Zugarmierung

$\bar{\varepsilon}$ e Auf die nominelle Fliessdehnung bezogene Dehnung in der Zugarmierung

$\varepsilon$ e max Maximale Dehnung in der Zugarmierung im Bereiche eines Risses

$\varepsilon$ emin Minimale Dehnung in der Zugarmierung im Bereiche eines Risses

$\varepsilon$ em Mittlere Dehnung in der Zugarmierung im Bereiche eines Risses

$\varepsilon_{f} \quad$ Nominelle Fliessdehmung der Zugarmierung

$\varepsilon$ plast Bleibende Stahldehnung

$\varepsilon$ eR Dehnung in der Zugarmierung in einem Rissequerschnitt

$\varepsilon$ er Dehnung in der Zugarmierung unmittelbar beim Beginn der Einschnürung

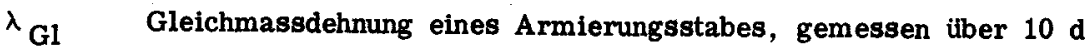
ausserhalb der Einschnürungszone

$\varepsilon_{b} \quad$ Betondehnung beziehungsweise -Stauchung

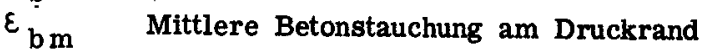

$\varepsilon_{\mathrm{b} \max }$ Maximale Betonstauchung am Druckrand

$\varepsilon_{\text {br }} \quad$ Bruchstauchung des Betons am Bruchrand (siehe Manuskript)

k Krümmung

$\mathrm{k}_{\mathrm{kr}}$ Krümmung beim Versagen der Zugarmierung

$\mathbf{k}_{\mathbf{k r b}} \quad$ Krümmung beim Versagen des Betons

$\gamma$ Schiebungswinkel bei Schubverformungen

$\delta_{i} \quad$ Neigung eines Biegeschubrisses gegenüber einer Senkrechten zur Balkenaxe

$\omega \quad$ Risswinkel: $\omega=\mathrm{w} /(\mathrm{h}-\mathrm{z})$

$\omega_{\mathrm{kr} \text { e Risswinkel beim Versagen der Zugarmierung }}$

$\omega_{\mathrm{krb}}$ Risswinkel beim Versagen des Betons

$\beta$ Drehwinkel in einem (elastischen oder plastischen) Gelenk des statisch bestimmten Grundsystems

$\theta \quad$ Gelenkwinkel in einem plastischen Gelenk

$\Theta_{\mathrm{el}} \quad$ Elastischer Anteil von $\boldsymbol{\theta}_{\mathrm{kr}}$

$\theta_{\text {er }} \quad$ Der zum Erreichen der Traglast erforderliche Gelenkwinkel

$\Theta_{\mathrm{kr}} \quad$ Gelenkwinkel beim Versagen im Gelenk (kritischer Gelenkwinkel) 


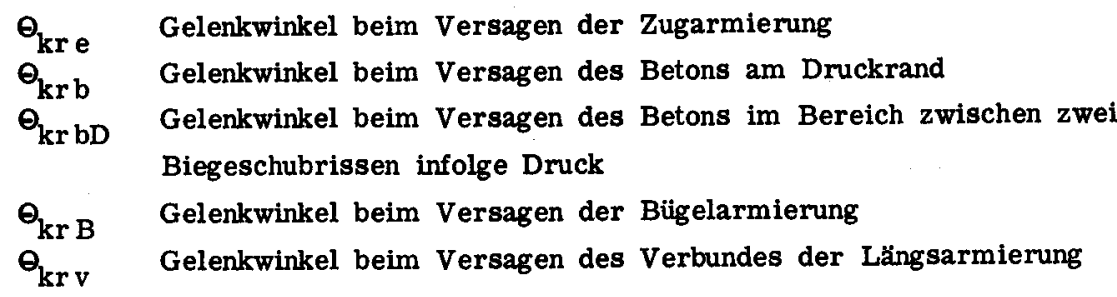

\section{Weitere dimensionslose Grössen}

\begin{tabular}{|c|c|}
\hline $\begin{array}{ll}c_{1}, c_{2} \\
k_{b}, & k_{b}\end{array}$ & $\begin{array}{l}\text { Konstanten zur Diskussion von } \mu_{\mathrm{Gr}} \\
\text { Konstanten zur Ermittlung der maximalen Betonrandstauchung in } \\
\text { Schubriss-Gelenken }\end{array}$ \\
\hline$\underset{\mathbf{k}^{\prime}}{\mathbf{k}_{\mathrm{R}}}$ & $\begin{array}{l}\text { Konstante zur Bestimmung des Risseabstandes } \\
\text { Exponent zur Variation der Betonrandstauchung im Bereiche eines } \\
\text { Biegerisses }\end{array}$ \\
\hline $\mathbf{i}$ & Lauf-Index \\
\hline $\mathrm{n}$ & $\begin{array}{l}\text { Anzahl Risse in einem plastischen Gelenk, in denen eine plastische } \\
\text { Verformung der Zugarmierung erfolgt, sowie }\end{array}$ \\
\hline $\mathbf{n}$ & Verhältnis der Elastizitätsmoduli von Stahl und Beton \\
\hline$\overline{\mathbf{m}}$ & Anzahl der plastischen Gelenke nach der Ausbildung eines Mechanismus \\
\hline$\overline{\mathbf{n}}$ & Grad der statischen Unbestimmtheit eines noch unbelasteten Tragwerks \\
\hline $\mathbf{r}$ & $\begin{array}{l}\text { Integralfunktion zur Berechnung der maximalen Relativverschiebung } v \\
\text { am Rissrand }\end{array}$ \\
\hline$\overline{\mathbf{r}}$ & Auf $\sigma_{f}$ und $\varepsilon_{f}$ bezogene Integralfunktion: $\bar{r}=r / \sigma_{f} \cdot \varepsilon_{f}$ \\
\hline $\mathbf{A}$ & Aufteilungsgrad einer die Biegedruckzone umschnürenden Bügelarmierung \\
\hline$\alpha$ & Matrix-Koeffizienten \\
\hline$\alpha^{\prime}, \alpha^{\prime \prime}$ & Matrix-Koeffizienten nach der Vornahme von Austauschschritten \\
\hline$\eta$ & Verhältnis zwischen innerem Hebelarm und statischer Nutzhöhe $(\eta=y / h)$ \\
\hline$\lambda$ & $\begin{array}{l}\text { Koeffizient zur Berücksichtigung der Abhängigkeit der Schubsteifigkeit } \\
\text { von der Beanspruchungshöhe }\end{array}$ \\
\hline$K$ & Verbundkoeff \\
\hline$\kappa^{*}$ & Verbundkoeffizient für $z / s=1,0$ \\
\hline
\end{tabular}


$\kappa^{\prime} \quad$ Koeffizient zur Variation der Betonrandstauchung im Bereiche eines Biegerisses

$\mu \quad$ Armierungsgehalt der Zugzone: $\mu=F / e^{/ b h}$

$\mu^{*} \quad$ Armierungsgehalt der Zugzone für $\mathrm{z} / \mathrm{s}=1,0$

$\mu_{\mathrm{Gr}} \quad$ Armierungsgehalt der Zugzone, bei dem gleichzeitig mit dem Versagen des Betons die Zugarmierung die nominelle Fliessspannung erreicht (Grenzarmierungsgehalt)

$\mu_{\mathrm{kr}} \quad$ Armierungsgehalt der Zugzone, bei dem Zugarmierung und Beton gleichzeitig versagen (Kritischer Armierungsgehalt)

$\mu^{\prime} \quad$ Armierungsgehalt der Druckzone: $\mu^{\prime}=F_{e}{ }^{1 / b h}$

$\mu_{q} \quad$ Querarmierungsgehalt der Blegedruckzone: $\mu_{q}=F_{q} /$ tc 


\section{LITERATUR - VERZEICHNIS}

[1] Prager W. : "Probleme der Plastizitätstheorie". Birkhẳuser-Verlag, Basel und Stuttgart 1955.

[2] Thürlimann B., Ziegler H. : "Plastische Berechnungsmethoden". Autographie der Vórlesungen vom Fortbildungskurs für Bau- und Maschineningenieure, ETH, Zürich 1963.

[3] American Society of Civil Engineers, Welding Research Council: "Commentary on Plastic Design in Steel". 1961.

[4] Wolfensberger R. : "Traglast und optimale Bemessung von Platten". Dissertation ETH, Institut für Baustatik, ETH, Zürich 1964.

[5] Anderheggen E.: "Optimale Bemessung von Stabtragwerken". Dissertation ETH, Institut für Baustatik, ETH, Zürich 1966.

[6] Bachmann H.: "Plastisches Verhalten statisch unbestimmter Stahlbetonbalken". Schweizerische Bauzeitung, 84. Jahrgang, Heft 41, Oktober 1966.

[7] Bachmann H., Thürlimann B.: "Versuche über das plastische Verhalten von zweifeldrigen Stahlbetonbalken, Serie A". Bericht Nr. 6203-1, Institut für Baustatik, ETH, Zürich, Juli 1965.

[ 8] Bachmann H., Thürlimann B.: "Versuche über das plastische Verhalten von zweifeldrigen Stahlbetonbalken, Serie B". Bericht Nr. 6203-2, Institut für Baustatik, ETH, Zürich, Dezember 1965.

[9] Bachmann H., Thürlimann B.: "Schubbemessung von Balken und Platten aus Stahlbeton, Stahlbeton mit Spannzulagen und Spannbeton". Schweizerische Bauzeitung, 84. Jahrgang, Hefte 33 und 34, August 1966.

[ 10] Maldague J . -C.: "Contribution à l'étude des déformations instantanées des poutres en béton armé". Annales de l'Institut Technique du Bátiment et des Travaux Publics, Paris, Septembre 1965.

[11] Djabry W.: "Contribution à l'etude de l'adhérence des fers d'armature au béton". EMPA-Bericht Nr. 184, Eidgenössische Materialprüfungs- und Versuchsanstalt, Zürich 1952.

[12] Rehm G.: "Ueber die Grundlagen des Verbundes zwischen Stahl und Beton". Deutscher Ausschuss für Stahlbeton, Heft Nr. 138, Berlin 1961.

[13] "Statische Zughaftfestigkeits-Ausziehversuche mit Rundeisen, Tor-Stahl 40, Caron-Stahl und Box-Stahl, d = $20 \mathrm{~mm}$ ". EMPA-Untersuchungs-Bericht Nr. 19798/1, Eidgenössische Materialprüfungs- und Versuchsanstalt, Zürich 1957 . 
[14] Baker A. L. L.: "Preliminary notes Nr. 1". Comité Europeen du Béton (CEB), Bulletin d'Information Nr. 21, Paris, Januar 1960.

[15] Base G. D., Read J. B.: "Effectiveness of Helical Binding in the Compression Zone of Concrete Beams". Journal of the American Concrete Institute, July 1965.

[16] Rüs ch H. , Stöckl S. : "Der Einfluss von Bügeln und Druckstäben auf das Verhalten der Biegedruckzone von Stahlbetonbalken". Deutscher Ausschuss für Stahlbeton, Heft Nr. 148, Berlin 1963.

[17] Rüisch H. : "Risstheorie". In "Vorträge auf dem Betontag 1965", S. 286. Verlag Deutscher Betonverein E. V.

[18] Comité Européen du Béton (CEB), Bulletin d'Information Nr. 24, Paris, Juni 1960.

[19] Sagels dorff R.: "Zur Haftfestigkeit von hochfesten Armierungsstählen". Schweizer Baublatt, Mai 1965.

[20] Le onhardt F.: "Spannbeton für die Praxis". 2. Auflage, S. $487 \mathrm{ff.,}$ Wilhelm Ernst \& Sohn, Berlin, 1962.

[21] Walters J.R.V., Mac Gregor J.G.: "An analytical study of inclined cracking in reinforced concrete beams". University of Alberta, Edmonton, Alberta, Canada, July 1966.

[22] Stiefel E.: "Einführung in die numerische Mathematik". 2. Auflage, B. G. Teubner Verlagsgesellschaft, Stuttgart 1963.

[23] Thürlimann B.: Vorlesung Baustatik II. Eidgenössische Technische Hochschule, Zürich.

[24] Leonhardt F., Walther R.: "Versuche an Plattenbalken mit hoher Schubbeanspruchung". Deutscher Ausschuss für Stahlbeton, Heft 152, Berlin 1962. 


\section{LEBENSLAUF}

Am 27. September 1935 in Olten (SO) geboren und daselbst aufgewachsen, trat ich 1952 in die Kantonsschule Solothurn ein. Nach der Maturitätsprififung nahm ich 1954 das Studium an der Eidgenössischen Technischen Hochschule in Zürich auf, wo ich 1959 - nach einjährigem Unterbruch infolge Militärdienst - mit dem Diplom als Bauingenieur abschloss. Wahrend mehr als 3 Jahren war ich alsdann in der Praxis tätig, vorerst als Statiker in einem Ingenieurbüro und anschliessend in der Entwicklungsabteilung einer führenden Firma auf dem Gebiete des Spannbetons und der Vorfabrikation. Seit dem Frühjahr 1963 bin ich als wissenschaftlicher Mitarbeiter von Herrn Prof. Dr. B. Thürlimann am Institut für Baustatik an der ETH angestellt. Hier befasste ich mich experimentell und theoretisch hauptsächlich mit den in dieser Arbeit behandelten Aspekten der Plastizitätstheorie, mit der Frage der Schubbemessung sowie mit weiteren Problemen aus dem Gebiete des Stahl- und Spannbetons. 
Seite Leer / Blank leaf 